Tank Waste Storage Ops.

3. From: (Originating Organization) Process Engineering, 74320

5. Proj./Prog./Dept./Div.:

Process Flowsheet Mode 17 ing/LMHC

8. Originator Remarks:

Approval/Release

6. Design Authority/Design Agent/Cog. Engr.:

L. W. Shelton, 376-6199
4. Related EDT No.:

NA

7. Purchase Order No.

NA

9. Equip./Component No.:

NA

10. System/Bldg./Facility:

NA

12. Major Assm. Dwg. No.:

NA

11. Receiver Remarks: 11A. Design Basel ine Document? [] Yes \& No

13. Permit/Permit Application No.: NA

14. Required Response Date:

NA

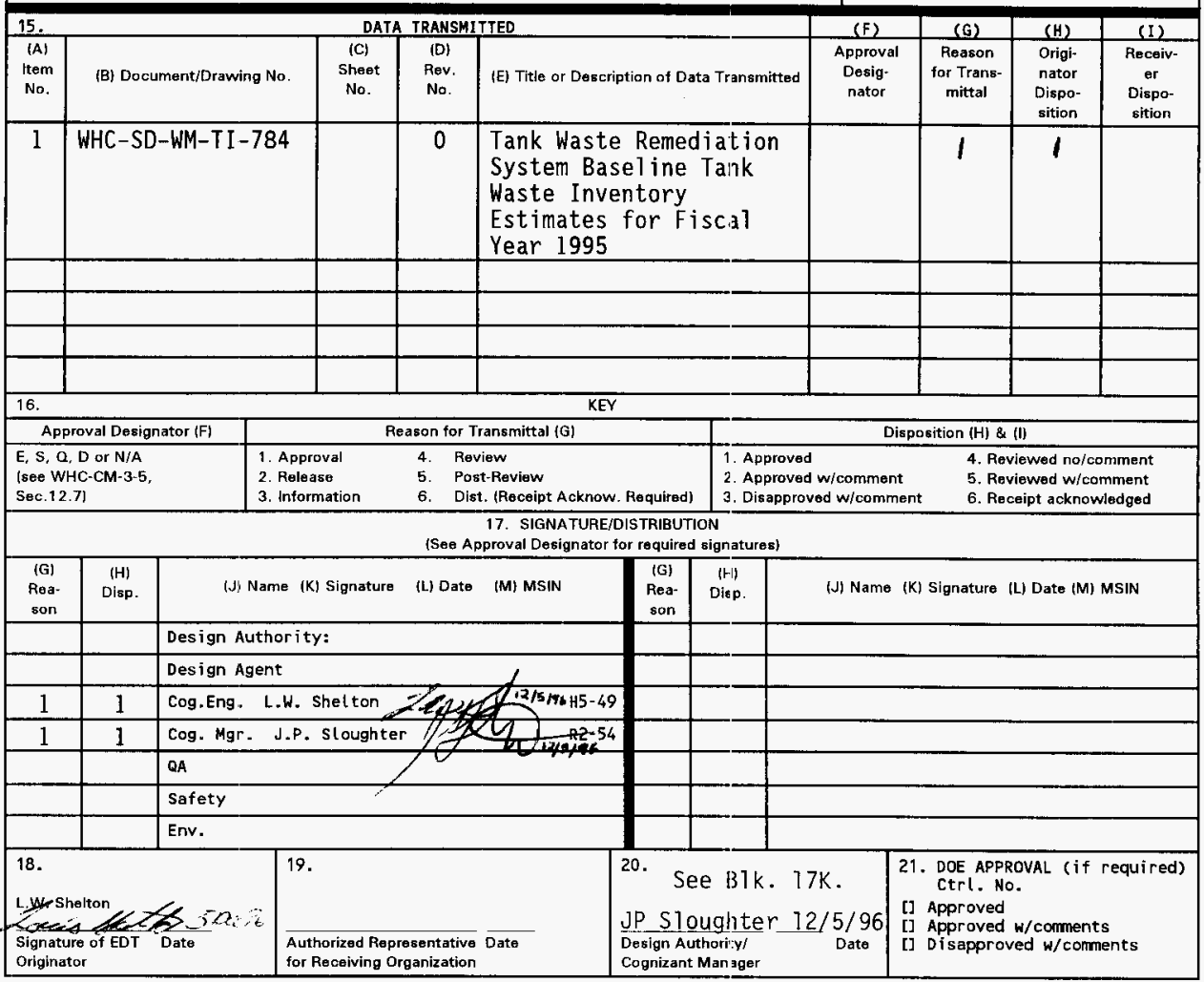

$B D-7400-172-2(05 / 96)$ GEF097 


\section{Tank Waste Remediation System Baseline Tank Waste Inventory Estimates for Fiscal Year 1995}

\section{W. Shelton}

Lockheed Martin Hanford Corp., Richland, WA 99352

U.S. Department of Energy Contract DE-AC06-87RL10930

EDT/ECN: $608755 \quad$ UC: 721

Org Code: 74320

Charge Code: D6222

B\&R Code: EW3130010

Total Pages: 111

Key Words: Inventory, waste, models

Abstract: A set of tank-by-tank waste inventories is derived from historical waste models, flowsheet records, and analytical data to support the Tank Waste Remediation System flowsheet and retrieval sequence studies. Enabling assumptions and methodologies used to develop the inventories are discussed. These provisional inventories conform to previously established baseline inventories and are meant to serve as an interim basis until standardized inventory estimates are made available.

TRADEMARK DISCLAIMER. Reference herein to any specific commercial product, process, or service by trade name, trademark, manufacturer, ог otherwise, does not necessarily constitute or imply its endorsement, recommendation, or favoring by the United States Government or any agency thereof or its contractors or subcontractors.

Printed in the United States of America. To obtain copies of this document, contact: WHC/BCS Document Control Services, P.O. Box 1970, Mailstop H6-08, Richland WA، 99352, Phone (509) 372-2420;

Fax (509) 376-4989.
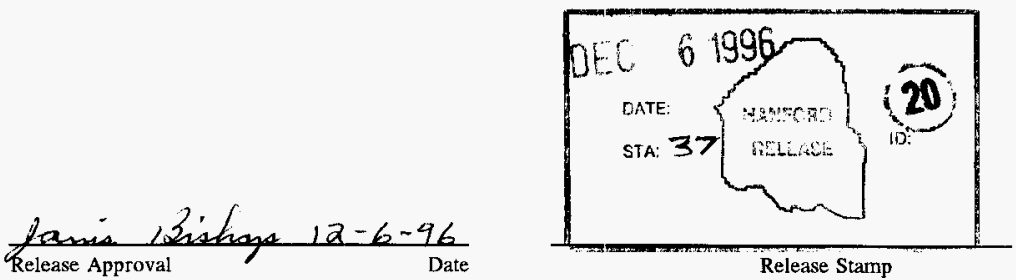

\section{Approved for Public Release}


WHC-SD-WM-TI-784

Revision 0

\title{
TANK WASTE REMEDIATION SYSTEM BASELINE TANK WASTE INVENTORY ESTIMATES FOR FISCAL YEAR 1995
}

September 1996

\author{
L. W. Shelton \\ Westinghouse Hanford Company \\ Richland, Washington
}

Prepared for

U.S. Department of Energy

Richland, Washington 
WHC-SD-WM-TI-784

Revision 0

This page intentionally left blank. 
WHC-SD-WM-TI-784

Revision 0

CONTENTS

1.0 INTRODUCTION $\ldots \ldots \ldots \ldots \ldots \ldots \ldots \ldots \ldots \ldots \ldots \ldots$

1.1 BACKGROUND ...................... 1

1.2 PURPOSE AND SCOPE $\ldots \ldots \ldots \ldots \ldots \ldots \ldots \ldots \ldots$

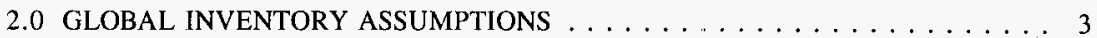

2.1 PREVIOUS INVENTORY ASSUMPTIONS . . . . . . . . . . . 3

2.2 CURRENT INVENTORY ASSUMPTIONS FOR SINGLE-SHELL TANKS . 5

2.3 CURRENT INVENTORY ASSUMPTIONS FOR DOUBLE-SHELL TANKS . 5

2.4 COMPARISON OF CURRENT GLOBAL INVENTORY ESTIMATES . . . . 5

3.0 DEVELOPMENT OF SINGLE-SHELL TANK ESTIMATES $\ldots \ldots \ldots \ldots \ldots 11$

3.1 CHEMICAL INVENTORIES FOR SINGLE-SHELL TANKS $\ldots \ldots \ldots 11$

3.1.1 Hanford Defined Waste Model . . . . . . . . . . . . . . 11

3.1.2 Normalizing Hanford Defined Waste Model Estimates to the Tank

Waste Remediation System Basis . . . . . . . . . . . . . . . . 11

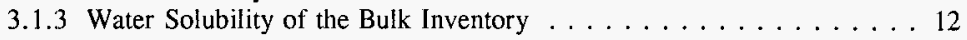

3.2 RADIONUCLIDE INVENTORIES FOR SINGLE-SHELL TANKS . . . . . . 19

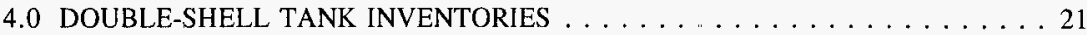

4.1 CHEMICAL INVENTORIES FOR DOUBLE-SHELL TANKS . . . . . 21

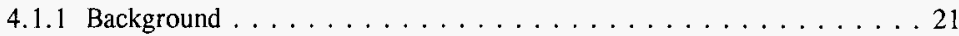

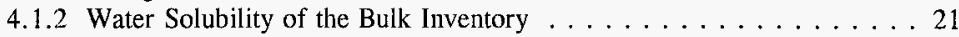

4.2 RADIONUCLIDE INVENTORIES FOR DOUBLE-SHELL TANKS $\ldots \ldots 25$

5.0 CONTRIBUTIONS FROM FUTURE FACILITY WASTES $\ldots \ldots \ldots . \ldots 29$

6.0 TOTAL INVENTORY FOR TANK WASTE REMEDIATION SYSTEM

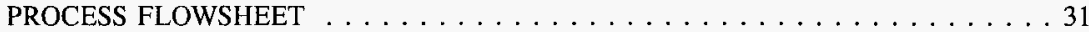

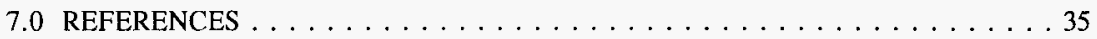

APPENDIX

A CHEMICAL INVENTORY ESTIMATES FOR SINGL_E-SHELL TANKS . . . . . A-1 
WHC-SD-WM-TI-784.

Revision 0

LIST OF FIGURES

2-1. Total Sodium in Single-Shell Tanks . . . . . . . . . . . . . . . 7

\section{LIST OF TABLES}

2-1. Comparison of Previous Inventory Estimates . . . . . . . . . . . . . 4

2-2. Comparison of Los Alamos National Laboratories SST Chemical Inventories with the 1987 Hanford Defense Waste Environmental Impact Statement . . . . . . . . . 9

3-1. Derivation of Salt Cake/Sludge Partitions for Single-Shell Tanks . . . . . . . . 13

3-2. Ratio of Salt Cake to Total Inventory in Single-Shell Tank Farms as Derived From the Hanford Defined Waste Model . . . . . . . . . . . . . . . . 14

3-3. Solubility Factors for Single-Shell Tanks $\ldots \ldots \ldots \ldots \ldots$

3-4. Results of Salt Cake Dissolution Studies . . . . . . . . . . . . . . . 17

3-5. Single-Shell Tank Chemical Inventory $\ldots \ldots \ldots \ldots \ldots \ldots$

4-1. Sludge Wash Factors for Double-Shell Tank Waste Tanks, . . . . . . . . . . 23

4-2. Double-Shell Tank Chemical Inventory $\ldots \ldots \ldots \ldots \ldots \ldots$

4-3. Comparison of Laboratory Data with Integrated Data Base Values for Double-Shell Tank Waste Types . . . . . . . . . . . . . . . . . . 26

4-4. Double-Shell Tank Radionuclide Inventory $\ldots \ldots \ldots \ldots \ldots \ldots$

$5-1$. Waste Contributions from Facilities . . . . . . . . . . . . . . 30

6-1. Chemical Inventory for Tank Waste Remediation Systems Reference Feed . . . . . 32

6-2. Radionuclide Inventory for Tank Waste Remediation Systems Reference Feed . . . . 34 


\section{LIST OF TERMS}

$\begin{array}{ll}\text { CC } & \text { Complexant concentrate } \\ \text { DST } & \text { Double-shell tank } \\ \text { EIS } & \text { Environmental Impact Statement } \\ \text { ERDA } & \text { Energy Research and Development Administration } \\ \text { HDW } & \text { Hanford Defined Waste } \\ \text { HDW-EIS } & \text { Hanford Defense Waste Environmental Inpact Statement } \\ \text { HDW-MDL } & \text { Hanford Defined Waste Model } \\ \text { HLW } & \text { High-level waste } \\ \text { HTCE } & \text { Historical tank content estimates } \\ \text { IDB } & \text { Integrated database } \\ \text { LANL } & \text { Los Alamos National Laboratory } \\ \text { LAW } & \text { Low-activity waste } \\ \text { LLW } & \text { Low-level waste } \\ \text { NCAW } & \text { Neutralized current aging waste } \\ \text { NCRW } & \text { Neutralized cladding removal waste } \\ \text { ORNL } & \text { Oak Ridge National Laboratories } \\ \text { OWVP } & \text { Operational Waste Volume Projection } \\ \text { PFP } & \text { Plutonium Finishing Plant } \\ \text { PUREX } & \text { Plutonium-Uranium Extraction (Process or Plant) } \\ \text { REDOX } & \text { Reduction and Oxidation (Process or Plant) } \\ \text { RIBD } & \text { Radioisotope Buildup and Decay } \\ \text { RHO } & \text { Rockwell Hanford Operations } \\ \text { SMM } & \text { Supernatant Mixing Model } \\ \text { SST } & \text { Single-shell tank } \\ \text { TLM } & \text { Tank Layer Model } \\ \text { TOC } & \text { Total organic carbon } \\ \text { TOR } & \text { Technical Options Report } \\ \text { TRAC } & \text { Track radioactive components } \\ \text { Tri-Party } & \text { Hanford Federal Facility Agreement and Consent Order } \\ \text { Agreement } & \\ \text { TWRS } & \text { Tank Waste Remediation System } \\ \text { TWRS-EIS } & \text { Tank Waste Remediation System Environmental Impact Statement } \\ \text { UR } & \text { Uranium recovery } \\ \text { WESF } & \text { Waste Encapsulation and Storage Facility } \\ \text { WSTRS } & \text { Waste status and transaction record summary } \\ & \end{array}$


WHC-SD-WM-TI-784.

Revision 0

This page intentionally left blank. 
WHC-SD-WM-TI-784

Revision 0

TANK WASTE REMEDIATION SYSTEM

BASELINE TANK WASTE INVENTORY ESTIMATES

FOR FISCAL YEAR 1995

\subsection{INTRODUCTION}

\subsection{BACKGROUND}

Radioactive waste, produced from previous reprocessing of irradiated uranium fuel from plutonium production reactors at the Hanford Site from 1944 to 1988, is currently stored in 28 clouble-shell tanks (DST) and 149 single-shell tanks (SST). The underground storage tanks are located in 18 tank farms in the 200 East and 200 West Areas of the Hanford Site. There is approximately $56 \mathrm{Mgal}$ of liquid and solid waste in these tanks. Terminal cleanout, decommissioning, and routine operations of facilities at the Hanford Site will, in the future, generate additional tank waste, mostly liquid.

Revision 1 of the TWRS Process Flowsheet (Orme 1995) for storage, treatment and immobilization of Hanford Site wastes utilizes a variety of simple separations to segregate the waste into a low-activity fraction containing the majority of the non-radioactive constituents, and a high-activity fraction. The low-activity waste (LAW) is vitrified and disposed of on the Hanford Site. The high-activity waste is also vitrified and stored, temporarily, on the Hanford Site before being shipped to a national geologic repository.

The Tank Waste Remediation System (TWRS) function includes characterization and retrieval of this tank waste for pretreatment. Hydraulic methods (sluicing and mixer pumps) are the preferred methods for retrieval of waste from both the SSTs and the DSTs. The order in which these tanks are retrieved is critical to developing a final waste form meeting all of the criteria spelled out in the Hanford Federal Facility Agreement and Consent Order (Tri-Party Agreement) (Ecology 1994). This is due to the high variance in waste composition from tank-to-tank combined with the need to maintain certain target feed compositions for the high-level waste (HLW) melter.

\subsection{PURPOSE AND SCOPE}

To support retrieval sequence studies and to provide a feed basis for the TWRS process flowsheet, a tank-by-tank inventory was derived from three principal sources: (1) tank-by-tank estimates developed by the Hanford Defined Waste Model (HDW-MDL) created at the Los Alamos National Laboratory (LANL) (Agnew 1995), (2) global inventory assessments developed for the 1987 Hanford Defense Waste Environmental Impact Statement (HDW-EIS) (RHO 1985), (3) analytical data from tank sarnpling activities. This TWRS 
inventory is divided into two fractions for each tank: (1) components that are in solution (soluble) and (2) components not in solution (insoluble).

The purpose of this report is to explain how the TWRS inventory was developed. Section 2.0 summarizes the development of earlier inventories, assesses their reliability, and explains how these inventory assumptions were used in the development of the current basis. Section 3.0 details the steps used to derive estimates of soluble and insoluble compositions for the SSTs. Section 4.0 does the same for DSTs.

It must be made clear at the outset that these tank-by-tank inventories were developed with the intent of making the combined inventory agree with previously established baselines. In doing so, difficulties in reconciling the total mass of a particular tank with available volume and density information often occur. The global and individual tank inventories presented here should be understood as an interim inventory basis until a more accurate assessment of the composition of the total waste inventory is made. 
WHC-SD-WM-TI-784

Revision 0

\subsection{GLOBAL INVENTORY ASSUMPTIONS}

\subsection{PREVIOUS INVENTORY ASSUMPTIONS}

There have been several attempts in the past to estimate chemical and radionuclide inventories of the Hanford Site's underground storage tanks. Values for key components from the various inventory estimates made to date are summarized in Table 2-1. The earliest estimates can be found in the Final Environmental Statement, Waste Management Operations, Hanford Reservation (ERDA 1975) and in Estimated Inventory of Chemicals Added to Underground Waste Tanks, 1944 through 1975 (Allen 1976). Although these inventory estimates are over twenty years old, they should account for most of the SST inventory since the majority of waste generated after 1975 was received by DSTs. Brief descriptions of the origin and development of these different inventory bases is discussed below.

The ERDA (1975) inventory is limited to just six sodium salts and it does not explain how the inventory was compiled. Allen's (1976) estimates are based on studies of the major chemical processes at the Hanford Site--their flowsheet compositions and recorded waste volumes sent to underground storage. Allen's report, being one of the earliest, seems to be the basis for subsequent inventory estimates for many components. However, recent research into Allen's work has uncovered several questionable assumptions made by Allen particularly in regard to flowsheet compositions and cribbing operations that impact total inventory estimates.

The compositions reported in Hanford Defense High-Level Waste Management Studies (Kaiser 1977) were derived from a "limited number of sample analysis and detailed records of fuel elements processed and chemicals used" (Kaiser 1977). The inventory reported in Technical Aspects of Long-Term Management Alternatives for High-Level Defense Waste at the Hanford Site (RHO 1980) is considered to be an update of the Kaiser (1977) report with sodium being the component that underwent the biggest change.

Of particular interest for the TWRS flowsheet is the inventory shown for SSTs in the data package for the 1987 Hanford Defense Waste Environmental Impact Statement (HDW-EIS) (RHO 1985). The chemical composition of Henford Site wastes in this document were derived from records of fuel elements processed, flowsheet records, and a limited number of waste sample analyses. For the development of this inventory, an effort was made to reconcile previously developed inventory assumptions (in particular Allen 1976 and RHO 1980) with independent reviews of flowsheets and purchase records. Radionuclide inventories in the HDW-EIS were taken largely from the Track Radioactive Components (TRAC) data (Jungfleisch 1984) with activation products being derived from hand calculations. The inventory totals listed in the HDW-EIS were used in the Technical Options Report (TOR) (Boomer et al. 1993) which provided the basis for the current TWRS inventory. 
The 1996 TWRS-EIS inventory is derived from the 1987 HDW-EIS for SSTs and available laboratory data for DSTs (Golberg and Guberski 1995). Unfortunately, the TWRS-EIS inventory and the TWRS flowsheet inventory for nonradioactive components are not identical. Adjustments made for aluminum, chloride, chromium, hydroxide, and phosphate in the TWRS inventory derived from the 1987 HDW-EIS were made after the 1995 TWRS-EIS data package was released. Changes in some of the DSTs made after receiving additional laboratory data also created minor differences between the TWRS-EIS and the TWRS flowsheet inventory. For radioactive components, the cumulative TWRS-EIS and TWRS inventory (DSTs plus SSTs) are identical. Golloerg mentions several adjustments from the HDW-EIS in the distribution of ${ }^{137} \mathrm{Cs},{ }^{99} \mathrm{Tc},{ }^{239 / 240} \mathrm{Pu}$, and ${ }^{241} \mathrm{Am}$ between DSTs, SSTs, and cesium capsules. These changes for radionuclides are reflected in the current TWRS flowsheet inventory.

Perhaps the most comprehensive effort to assess waste compositions for all 177 underground storage tanks thus far has come from LANL. Created at LANL, the HDWMDL is a detailed historical model that uses information from process flowsheets, purchase records, sample data, and historical transaction records to arrive at final tank compositions (Agnew 1995). The HDW-MDL, along with sample data, promises to be a very important tool for developing definitive tank compositions. The tank-by-tank composition estimates generated by the HDW-MDL for the SSTs provided the starting point for the current TWRS inventory in that the HDW-MDL estimates in every SST were adjusted in direct proportion to each other on a component by component basis so that the totals reported for SSTs were the same as those reported in the HDW-EIS.

Table 2-1. Comparison of Previous Inventory Estimates.

\begin{tabular}{|l|c|c|c|c|c|c|c|c|}
\hline Report & $\begin{array}{c}\text { Allen } \\
1976\end{array}$ & $\begin{array}{c}\text { Kaiser } \\
1977\end{array}$ & $\begin{array}{c}\text { RHO } \\
1980\end{array}$ & $\begin{array}{c}\text { RHO } \\
1985\end{array}$ & $\begin{array}{c}\text { Boomer } \\
1993\end{array}$ & $\begin{array}{c}\text { Golberg } \\
1995\end{array}$ & $\begin{array}{c}\text { Agnew } \\
1995\end{array}$ & $\begin{array}{c}\text { TWRS } \\
1995\end{array}$ \\
\hline \multicolumn{1}{|c|}{ Date } & $3 / 76$ & $9 / 77$ & $9 / 80$ & $12 / 85$ & $3 / 93$ & $7 / 95$ & $9 / 95$ & $8 / 95$ \\
\hline & $(\mathrm{MT})$ & $(\mathrm{MT})$ & $(\mathrm{MT})$ & $(\mathrm{MT})$ & $(\mathrm{MT})$ & $(\mathrm{MT})$ & $(\mathrm{MT})$ & $(\mathrm{MT})$ \\
\hline $\mathrm{Al}^{+3}$ & $1.08 \mathrm{E}+04$ & $4.34 \mathrm{E}+03$ & $4.64 \mathrm{E}+03$ & $2.66 \mathrm{E}+03$ & $4.64 \mathrm{E}+03$ & $4.17 \mathrm{E}+03$ & $7.07 \mathrm{E}+03$ & $4.83 \mathrm{E}+03$ \\
\hline $\mathrm{Bi}^{+3}$ & $2.51 \mathrm{E}+02$ & $2.82 \mathrm{E}+02$ & $2.61 \mathrm{E}+02$ & $2.61 \mathrm{E}+02$ & $2.61 \mathrm{E}+02$ & $2.64 \mathrm{E}+02$ & $6.75 \mathrm{E}+02$ & $2.61 \mathrm{E}+02$ \\
\hline $\mathrm{Ca}^{+2}$ & $1.04 \mathrm{E}+02$ & $1.36 \mathrm{E}+02$ & $1.28 \mathrm{E}+02$ & $1.28 \mathrm{E}+02$ & $1.45 \mathrm{E}+02$ & $1.50 \mathrm{E}+02$ & $6.45 \mathrm{E}+02$ & $1.65 \mathrm{E}+02$ \\
\hline $\mathrm{Cl}^{-}$ & $4.30 \mathrm{E}+01$ & $3.60 \mathrm{E}+01$ & $4.00 \mathrm{E}+01$ & $4.00 \mathrm{E}+02$ & $4.86 \mathrm{E}+02$ & $3.14 \mathrm{E}+02$ & $6.24 \mathrm{E}+02$ & $4.89 \mathrm{E}+02$ \\
\hline $\mathrm{Cr}^{+3}$ & $7.18 \mathrm{E}+02$ & $3.63 \mathrm{E}+02$ & $3.43 \mathrm{E}+02$ & $9.60 \mathrm{E}+01$ & $1.64 \mathrm{E}+02$ & $1.83 \mathrm{E}+02$ & $8.27 \mathrm{E}+02$ & $1.65 \mathrm{E}+02$ \\
\hline $\mathrm{F}^{-}$ & $8.1 \mathrm{E}+02$ & $\mathrm{~N} / \mathrm{A}$ & $\mathrm{N} / \mathrm{A}$ & $8.23 \mathrm{E}+02$ & $1.19 \mathrm{E}+03$ & $1.18 \mathrm{E}+03$ & $9.20 \mathrm{E}+02$ & $1.17 \mathrm{E}+03$ \\
\hline $\mathrm{Fe}^{+3}$ & $1.26 \mathrm{E}+03$ & $7.15 \mathrm{E}+02$ & $7.09 \mathrm{E}+02$ & $7.87 \mathrm{E}+02$ & $8.21 \mathrm{E}+02$ & $7.77 \mathrm{E}+02$ & $2.79 \mathrm{E}+03$ & $8.21 \mathrm{E}+02$ \\
\hline $\mathrm{Na}^{+}$ & $6.26 \mathrm{E}+04$ & $5.88 \mathrm{E}+04$ & $6.07 \mathrm{E}+04$ & $6.18 \mathrm{E}+04$ & $6.85 \mathrm{E}+04$ & $6.91 \mathrm{E}+04$ & $4.05 \mathrm{E}+04$ & $6.83 \mathrm{E}+04$ \\
\hline $\mathrm{PO}_{4-3}$ & $8.66 \mathrm{E}+03$ & $8.97 \mathrm{E}+03$ & $8.90 \mathrm{E}+03$ & $9.34 \mathrm{E}+03$ & $8.96 \mathrm{E}+03$ & $4.97 \mathrm{E}+03$ & $4.64 \mathrm{E}+03$ & $5.78 \mathrm{E}+03$ \\
\hline $\mathrm{Si}^{+4}$ & $4.18 \mathrm{E}+02$ & $4.61 \mathrm{E}+02$ & $4.47 \mathrm{E}+02$ & $4.47 \mathrm{E}+02$ & $5.06 \mathrm{E}+02$ & $5.31 \mathrm{E}+02$ & $4.83 \mathrm{E}+02$ & $5.06 \mathrm{E}+02$ \\
\hline $\mathrm{SO}_{4}$ & $5.34 \mathrm{E}+03$ & $6.90 \mathrm{E}+02$ & $7.17 \mathrm{E}+02$ & $1.77 \mathrm{E}+03$ & $2.03 \mathrm{E}+03$ & $2.040 \mathrm{E}+03$ & $5.50 \mathrm{E}+03$ & $2.04 \mathrm{E}+03$ \\
\hline $\mathrm{Zr}^{+4}$ & $2.46 \mathrm{E}+02$ & $2.65 \mathrm{E}+02$ & $2.77 \mathrm{E}+02$ & $2.77 \mathrm{E}+02$ & $5.99 \mathrm{E}+02$ & $5.24 \mathrm{E}+02$ & $3.06 \mathrm{E}+02$ & $6.39 \mathrm{E}+02$ \\
\hline
\end{tabular}




\subsection{CURRENT INVENTORY ASSUMPTIONS FOR SINGLE-SHELL TANKS}

The current TWRS inventory still uses the SST totals shown in HDW-EIS with the following exceptions: aluminum was increased from $2.66 \mathrm{E}+06 \mathrm{~kg}$ to $2.96 \mathrm{E}+06 \mathrm{~kg}$ based on a study made by Borsheim (1994), chloride was increased from $4.00 \mathrm{E}+04 \mathrm{~kg}$ to 4. $15 \mathrm{E}+05$ to account for chloride impurities in $\mathrm{NaOH}$, chromium was increased from $9.60 \mathrm{E}+04 \mathrm{~kg}$ to $2.68 \mathrm{E}+05 \mathrm{~kg}$ because of a error made while transcribing data from RHO 1980 to RHO 1985, hydroxide was increased from $9.14 \mathrm{E}+06 \mathrm{~kg}$ to $1.27 \mathrm{E}+07 \mathrm{~kg}$ to achieve a charge balance, and phosphate was decreased from $8.74 \mathrm{E}+06 \mathrm{~kg}$ to $4.73 \mathrm{E}+06 \mathrm{~kg}$ to account for mistaken assumptions about the amount of phosphate that was sent directly to the ground through cribbing operations (Boldt 1994).

The TRAC data served as the basis for the SST radionuclide inventory with some revision as outlined in the data package for the 1996 TWR'S-EIS (Golberg 1995). The combined TWRS-EIS radionuclide values for SSTs and DSTs were adopted for the TWRS flowsheet inventory but the distribution between SSTs and DSTs were modified in light of more recent sample data for DSTs.

\subsection{CURRENT INVENTORY ASSUMPTIONS FOR DOUBLE-SHELL TANKS}

For DSTs, the previous flowsheet basis relied primarily on results obtained from the latest laboratory data along with some independent studies that attempt to reconcile laboratory data with process knowledge and flowsheet records. The TWRS inventory reflects the same approach as the previous basis but a larger set of DST sample data was available for use. Laboratory data were the primary sources of information for chemicals. This is also true largely for radionuclides although some flowsheet records and plant tracking data was used for several radioisotopes in some waste types. Also, some averaging was needed for ${ }^{99} \mathrm{Tc}$ in tanks lacking ${ }^{99} \mathrm{Tc}$ data.

Laboratory data for DSTs are more reliable than SSTs for two principal reasons. First, a larger percentage of DST waste is in the liquid phase thus reducing the error associated with the heterogeneity of solid waste. Secondly, the sludges in the DSTs are largely segregated (i.e., the mixing of waste types in the DSTs has not occurred to the same extent as the SSTs), therefore, the sludges in the DSTs are more representative of a single waste type and not subject to heterogeneity issues to the same degree as the SSTs.

\subsection{COMPARISON OF CURRENT GLOBAL INVENTORY ESTIMATES}

In addition to these discrepancies, there are several questionable component totals in the HDW-EIS and other inventory estimates compiled to date. The most important of these is sodium. Sodium is one of the most important components in the waste in that it will determine the size and processing rates for the (Low Activity Waste) LAW facility. The previous inventory basis (Boomer et al. 1993) quotes a SST sodium inventory of 
$5.13 \mathrm{E}+07 \mathrm{~kg}$ after the completion of saltwell pumping. Adding the saltwell liquid back to the SSTs increases this number to $5.73 \mathrm{E}+07 \mathrm{~kg}$ of sodium. For comparison, the HDWMDL predicts a sodium inventory of only $3.00 \mathrm{E}+07 \mathrm{~kg}$ in the SSTs or 51 percent of the previous inventory basis. Preliminary aggregate sodium estimates made by the HDW-MDL for the DSTs were very close to the total sodium obtained by analysis of DST sample data.

With a reported volume of $1.36 \mathrm{E} 5 \mathrm{~m}^{3}$ of SST waste (Hanlon 1996), the bulk sodium molarity in SSTs would be $18.5 \mathrm{M}$ using the $5.73 \mathrm{E}+07 \mathrm{~kg}$ value, but this assumes that the SST waste is monolithic. To be more accurate, it is necessary to look at the sludge, salt cake, and liquid fractions separately. There is an estimated $4.59 \mathrm{E}+04 \mathrm{~m}^{3}$ of sludge, $8.80 \mathrm{E}+04 \mathrm{~m}^{3}$ of salt cake, and 2.085E $+03 \mathrm{~m}^{3}$ of supernatant in the SSTs (Hanlon 1996). Assuming an average sodium molarity in the sludge of 5.25 (based on weighted average of 27 core samples) (Colton 1995), the corresponding salt cake molarity would have to be $25.6 M$ sodium in order to arrive at $5.73 \mathrm{E}+07 \mathrm{~kg}$ of sodium.

Although unlikely, this situation is not physically impossible since a pure $\mathrm{NaNO}_{3}$ salt cake with a bulk density of 2.26 , has a sodium concentration close to $31 M$. However, the salt cake in the waste tanks contains considerable void spaces that may or may not contain liquid, liquid at a much lower sodium concentration than $25.6 \mathrm{M}$, indeed sodium concentrations in the interstitial liquid are not expected to exceed 12M (Agnew and Watkin 1995). Porosity is the term used to describe the void spaces in the salt cake layer. It is defined as the percentage of salt cake volume not occupied by crystallized salts. The baseline porosity value used in the operational waste volume projection model at the time was 61 vol\% - up from 45 percent used in previous waste volume projections (Strode 1995). Whether the average porosity of the salt cake is $61 \mathrm{vol} \%$ or $45 \mathrm{vol} \%$, a total inventory of over 57,000 MT as stated in the previous basis, is very unlikely for the SSTs.

Figure 2-1 represents a more detailed analysis of SST sodium data. Salt cake, supernatant, and sludge volumes were taken from Hanlon (1996). The sodium concentration in the sludge was assumed to be $5.26 \mathrm{M}$ based on a weighted average of 27 core samples (Colton 1995). The dry salt cake was assumed to be 100 percent $\mathrm{NaNO}_{3}$. The graph was generated by varying the porosity of the salt cake in the SSTs and the concentration of sodium in the liquid phase between $8 M$ and $12 M$--boundaries determined by analysis of supernate samples (Agnew and Watkins 1995).

The bounding concentrations and bounding porosity values shown in Figure 2-1 indicates that the range for total sodium in SSTs is $3.50 \mathrm{E}+07$ to $4.50 \mathrm{E}+07 \mathrm{~kg}$ for SSTs. The HDW-MDL predicts a SST sodium inventory of $2.97 \mathrm{E}+07 \mathrm{~kg}$ while the previous inventory basis for the TWRS flowsheet is $5.73 \mathrm{E}+07 \mathrm{~kg}$. 


\section{WHC-SD-WM-TI-784 \\ Revision 0}

Figure 2-1. Total Sodium in Single-Shell Tanks.

(at different salt cake porosities and superrate concentrations)

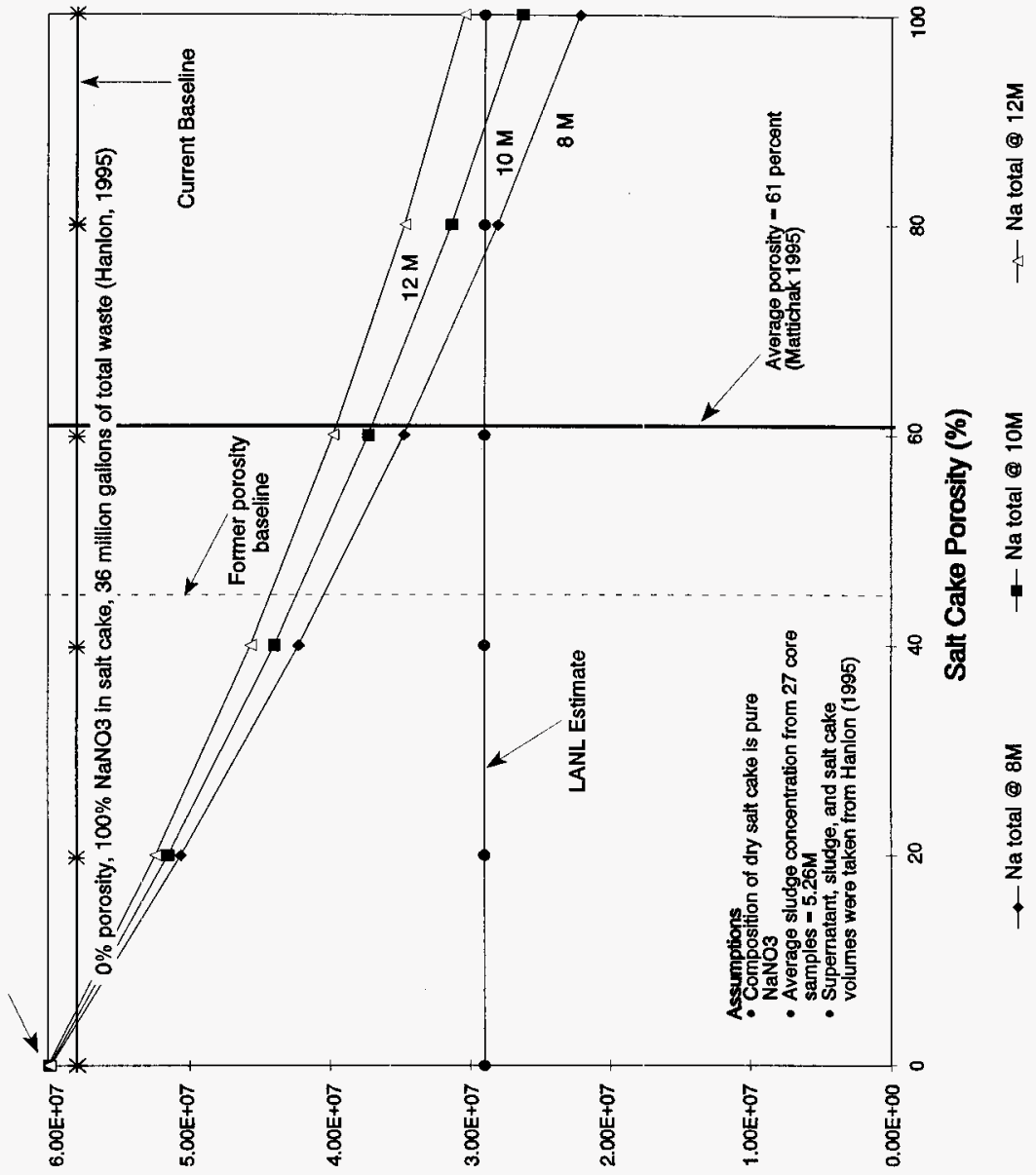

(6y) un!̣poS LSS IEłoI 


\section{Revision 0}

It appears that the sodium value in the previous TWRS inventory basis is biased high. Like many component inventory estimates, the sodium estimate is traceable back to Allen (1976). Allen apparently overstated the sodium inventory mostly by assuming 83 percent less sodium from the $\mathrm{BiPO}_{4}$ process was sent to cribs and estimating an amount of sodium added through the uranium recovery process (UR) that is 45 percent higher than the HDW-MDL prediction.

Along with sodium, the nitrate inventory used previously may be overstated by more than a factor of two. The HDW-MDL predicts there to be: $4.28 \mathrm{E}+07 \mathrm{~kg}$ of nitrate as opposed to $1.00 \mathrm{E}+08 \mathrm{~kg}$ in the previous inventory basis. Preliminary efforts at reconciliation of these two numbers suggest that the HDW-MDL is once again the more realistic figure (Kupfer 1996a).

Other components listed in the HDW-EIS with inventory values markedly different than the HDW-MDL that could impact immobilized waste form volumes and plant design include chromium, iron, and sulfate. A comparison of the differences for these components and other major components as well is shown in Table 2-2.

A managerial decision was made to retain the global inventories reported in the HDW-EIS because the HDW-MDL estimates and preliminary attempts to reconcile the HDW-MDL with other inventory estimates had not been validated or verified. It was, therefore, deemed more important to retain consistency wi:h the past until a thorough review and verification process has established a new baseline.

The process for defining a standard inventory estimate began near the start of fiscal year (FY) 1996 (Kupfer 1996b). Substantial progress has already been made, but final global inventory estimates are not expected to be released until August 1997.

Section 3.0 details how tank-by-tank chemical compositions provided by the HDW-MDL were used to arrive at individual SST tank inventories. 
Table 2-2. Comparison of Los Alamos National Laboratories SST Chemical Inventories with the 1987 Hanford Defense Waste Environinental Impact Statement.

\begin{tabular}{|c|c|c|c|}
\hline Component & $\begin{array}{c}\text { Los Alamos National } \\
\text { Laboratory (LANL) (MT) }\end{array}$ & $\begin{array}{l}1987 \text { Environmental Impact } \\
\text { Statement (EIS) (MT) }\end{array}$ & $\begin{array}{c}\text { LANL/1987 } \\
\text { EIS } \\
\end{array}$ \\
\hline $\mathrm{Al}^{+3}$ & $5.26 \mathrm{E}+03$ & $2.96 \mathrm{E}+03^{(\mathrm{a})}$ & 1.78 \\
\hline $\mathrm{Bi}^{+3}$ & $6.64 \mathrm{E}+02$ & 2.61. $\mathrm{E}+02$ & 2.54 \\
\hline Cancrinite & $1.94 \mathrm{E}+03$ & $2.70 \mathrm{E}+03$ & 0.718 \\
\hline $\mathrm{Ca}^{+2}$ & $5.36 \mathrm{E}+02$ & $1.28 \mathrm{E}+02$ & 4.18 \\
\hline $\mathrm{Cl}^{-}$ & $3.50 \mathrm{E}+02$ & 4.15E+02 & 0.843 \\
\hline $\mathrm{CO}^{-2}$ & $2.73 E+03$ & $1.66 \mathrm{E}+03$ & 1.64 \\
\hline $\mathrm{Cr}^{+3}$ & $7.47 \mathrm{E}+02$ & $2.68 \mathrm{E}+02^{(\mathrm{b})}$ & 2.79 \\
\hline $\mathrm{F}^{-}$ & $5.39 \mathrm{E}+02$ & 8.12E+02 & 0.663 \\
\hline $\mathrm{Fe}^{+3}$ & $2.59 \mathrm{E}+03$ & $6.31 \mathrm{E}+02$ & 4.10 \\
\hline $\mathrm{K}^{+}$ & $1.31 \mathrm{E}+02$ & 5.5 $\mathrm{E}+01$ & 2.37 \\
\hline $\mathrm{La}^{+3}$ & $4.01 \mathrm{E}+01$ & $1.88 \mathrm{E}+00$ & 21.3 \\
\hline $\mathrm{Mn}^{+4}$ & $1.75 \mathrm{E}+01$ & $1.20 \mathrm{E}+02$ & 0.146 \\
\hline $\mathrm{Na}^{+}$ & $2.97 \mathrm{E}+04$ & $5.7 \mathrm{E}+04$ & 0.513 \\
\hline $\mathrm{Ni}^{+3}$ & $2.10 \mathrm{E}+02$ & $2.0 \mathrm{E} \mathrm{E}+02$ & 1.03 \\
\hline $\mathrm{NO}^{2}$ & $5.57 \mathrm{E}+03$ & $6.52 \mathrm{E}+03$ & 0.852 \\
\hline $\mathrm{NO}^{-}$ & $4.28 \mathrm{E}+04$ & $1.00 \mathrm{E}+05$ & 0.426 \\
\hline $\mathrm{OH}^{-}$ & $1.70 \mathrm{E}+04$ & $1.06 \mathrm{E}+04$ & 1.60 \\
\hline $\mathrm{Pb}^{+4}$ & $1.32 \mathrm{E}+01$ & $2.83 \mathrm{E}+01$ & 0.466 \\
\hline $\mathrm{PO}_{4}^{-3}$ & $3.91 \mathrm{E}+03$ & $4.73 \mathrm{E}+03$ & 0.827 \\
\hline $\mathrm{Si}^{+4}$ & $9.41 \mathrm{E}+01$ & $1.45 \mathrm{E}+01$ & 6.49 \\
\hline $\mathrm{SO}^{-2}$ & $4.32 \mathrm{E}+03$ & $1.65 \mathrm{E}+03$ & 2.62 \\
\hline $\mathrm{Sr}^{+2}$ & $1.57 \mathrm{E}+02$ & $3.60 \mathrm{E}+01$ & 4.36 \\
\hline $\mathrm{UO}_{2}^{+2}$ & $1.92 \mathrm{E}+03$ & $1.61 \mathrm{E}+03$ & 1.19 \\
\hline $\mathrm{Zr}^{+4}$ & $9.36 \mathrm{E}+01$ & $3.82 \mathrm{E}+02$ & 0.245 \\
\hline TOC & $2.99 \mathrm{E}+02$ & $4.73 E+02$ & 0.633 \\
\hline TOTAL & $1.22 \mathrm{E}+05$ & $1.94 \mathrm{E}+05$ & 0.629 \\
\hline
\end{tabular}

${ }^{a}$ Adjusted from 2.21E $+03 \mathrm{MT}$ in the RHO (1985). Does not include aluminum found in cancrinite, $2 \mathrm{NaAlSiO}_{4}: 0.52 \mathrm{NaNO}_{3}: 0.68 \mathrm{H}_{2} \mathrm{O}$.

${ }^{\mathrm{b}}$ Adjusted from $9.59 \mathrm{E}+01 \mathrm{MT}$ in the RHO (1985). 
WHC-SD-WM-TI-784

Revision 0

This page intentionally left blank. 
WHC-SD-WM-TI-784

Revision 0

\subsection{DEVELOPMENT OF SINGLE-SHELL TANK ESTIMATES}

\subsection{CHEMICAL INVENTORIES FOR SINGLE-SHELLL TANKS}

\subsubsection{Hanford Defined Waste Model}

The tank-by-tank distribution of components in the HDW-MDL developed at LANL provided the starting point for the derivation of the current TWRS inventory. The HDW-MDL provides estimates for 35 chemicals and four radionuclides $\left({ }^{137} \mathrm{Cs},{ }^{239 / 240} \mathrm{Pu}\right.$, ${ }^{90} \mathrm{Sr}$, and ${ }^{238} \mathrm{U}$ ). The emphasis in the HDW-MDL is on chemicals. Relatively simplistic assumptions and correlations were used to estimate radionuclide quantities. Therefore, only the distribution of chemicals in the HDW-MDL for the SSTs were used to develop the current TWRS inventory.

At the time the TWRS inventory was being developed, LANL had released the first revision of their inventory estimates for the SSTs to ICF Kaiser who later published them as the Historical Tank Content Estimates (HTCE) (Brevick et al. 1995a, b, c, Brevick 1995). At that time, supernatant estimates had not been made as their Supernatant Mixing Model (SMM) was not yet complete. Since supernatant occupies less than $2.0 \mathrm{vol} \%$ of the total SST waste volume, its omission does not greatly effect the inventory estimates. Nor did the HDW-MDL include any information about additions of diatamaceous earth to the SSTs.

\subsubsection{Normalizing Hanford Defined Waste Model Estimates to the Tank Waste Remediation System Basis}

In Table 2-2, the global inventory estimates for SSTs used in the HDW-EIS were compared with the respective totals calculated by Revision 1 of the HDW-MDL. For the current TWRS inventory development, the HDW-MDL estimates for each component in every SST were multiplied by the ratio of the HDW-EIS values (the previous baseline) to the HDW-MDL values shown in column four of Table 2-2 so that the sum of the SSTs equaled the previous baseline global inventories. This almost doubles both the sodium and nitrate numbers. Since sodium and nitrate comprise the bulk of the waste in the tanks, doubling them creates difficulties in reconciling tank masses with rejorted volumes when using typical bulk densities.

One component not reported by the HDW-MDL but included in Table 2-2 is cancrinite, $2 \mathrm{NaAlSiO}_{4}: 0.52 \mathrm{NaNO}_{3}: 0.68 \mathrm{H}_{2} \mathrm{O}$. Cancrinite is used in the TWRS flowsheet to represent the water insoluble sodium aluminosilicates that are expected to be found in most tank sludges. In the HDW-MDL, the sodium, aluminum, silicon, and nitrate concentrations were reduced stoichiometrically in each tank to "form" cancrinite using silicon as the limiting species unless the aluminum was exhausted first. This method ignores the distribution of waste 


\section{Revision 0}

between salt cake and sludge; cancrinite should not form from salt cake. However, silicon is expected to predominate in the sludge rather than the salt cake so the assumption has validity.

\subsubsection{Water Solubility of the Bulk Inventory}

The HDW-MDL provides bulk inventories only. After normalizing the HDW-MDL inventories to the HDW-EIS totals, an estimate of the effect of water-washing on the waste must be made in order to use this information in the TWRS flowsheet models. To do this, the waste in each tank must first be divided into salt cake and sludge partitions, then dissolution factors obtained from sludge washing data and water washing of salt cake are applied to the sludge and salt cake partitions.

The 50 waste types defined by LANL in Agnew (1995) are used to divide the content of each SST into a salt cake and sludge fraction. The defined waste list includes nine salt cake/salt-slurry types and 41 sludge types. Spreadsheets containing the compositions of these waste types and the volumes of each waste type in every tank were provided by LANL. For each tank the salt cake layers and sludge layers were summed separately and then the percentage of each component in a tank that is salt cake was calculated. For example, consider the excerpt from the spreadsheets obtained from LANL shown in Table 3-1.

Four of the 50 waste types are shown, three represerit sludges and one (T2-SltCk) is a salt cake stream. Below these compositions are the volumes of each waste type that went into four tanks in 241-TX farm, 241-TX-101 through 241-TX-104. According to LANL these four waste types are the only types expected to be found in the four tanks. Based on Table 3-1, the percentage of sodium in the salt cake in Tank 241-TX-101 would be calculated as follows by dividing the amount of sodium in the salt calke streams by the total mass of sodium in all four streams:

$$
\begin{gathered}
\text { Percent Na in sltck }=\left[(8)^{*}(13.5) /\left((3) *(4.8)+(70)^{*}(2.3)+(3)\right.\right. \\
*(3.4)+(8) *(13.5))] / 100=36.8 \text { percent }
\end{gathered}
$$

Once obtained, the salt cake percentage calculated above for sodium is multiplied by the total sodium distributed to the tank by the HDW-MDL normalized to the HDW-EIS. The process is repeated for each component of interest in every SST. Table 3-2 shows the ratio of salt cake to the total inventory for each SST farm resulting from this process. 


\section{WHC-SD-WM-TI-784}

Revision 0

Table 3-1. Derivation of Salt Cake/Sludge Partitions for Single-Shell Tanks. ${ }^{{ }^{*}}$

\begin{tabular}{|c|c|c|c|c|}
\hline Sludge, $\mathrm{mol} / \mathrm{L}$ & MW $44-51^{\mathrm{b}}$ & $\mathrm{R}^{\prime} 52-58^{\mathrm{c}}$ & $\mathrm{Z} / \mathrm{PFP}^{\mathrm{d}}$ & T2-SltCk \\
\hline $\mathrm{Na}$ & 4.8 & 2.3 & 3.4 & 13.5 \\
\hline $\mathrm{Al}$ & 0 & 4.6 & 3.3 & 1.1 \\
\hline $\mathrm{Fe}$ & 0.32 & 0.51 & 0.49 & 0.016 \\
\hline $\mathrm{Cr}$ & 0.0041 & 0.46 & 0.0083 & 0.12 \\
\hline $\mathrm{NO}_{3}$ & 0.13 & 2.1 & 2.5 & 10.4 \\
\hline $\mathrm{NO}_{2}$ & 0 & 0 & 0.012 & 1.07 \\
\hline $\mathrm{PO}_{4}$ & 0.47 & 0 & 0.00012 & 0.035 \\
\hline Tank & $\begin{array}{c}\text { MW 44-51 } \\
\text { (kgal) }\end{array}$ & $\begin{array}{c}\text { R'52-58 }^{\prime} 5 \text { (kgal) } \\
\end{array}$ & $\begin{array}{l}\text { Z/PFP } \\
\text { (kgal) }\end{array}$ & $\begin{array}{c}\text { T2-SltCk } \\
\text { (kgal) }\end{array}$ \\
\hline TX-101 & 3 & 70 & 3 & 8 \\
\hline $\mathrm{TX}-102$ & 2 & 0 & 0 & 111 \\
\hline $\mathrm{TX}-103$ & 3 & 0 & 0 & 154 \\
\hline TX-104 & 18 & 0 & 0 & 46 \\
\hline
\end{tabular}

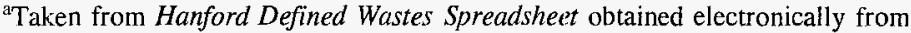
LANL in August 1995.

'Metal Waste produced between 1944 and 1951.

'REDOX waste produced between 1952 and 1953.

'Derived from analysis of waste in DST 241-SY.-102.

eSalt cake taken from 242-T, 1965-1976, TX-118 feed. 


\begin{tabular}{|c|c|c|c|c|c|c|c|c|c|c|c|c|c|}
\hline Component & A & $\mathrm{AX}$ & B & $\mathrm{BX}$ & BY & $\mathrm{C}$ & $S$ & SX & $\mathrm{T}$ & $\mathrm{TX}$ & TY & $\mathrm{U}$ & Total \\
\hline $\mathrm{Al}^{+3}$ & 1.00 & 1.00 & 0.04 & 0.15 & 0.97 & 0.00 & 0.96 & 0.92 & 0.11 & 0.95 & 0.44 & 0.85 & 0.62 \\
\hline $\mathrm{Bi}^{+3}$ & 1.00 & 1.00 & 0.04 & 0.00 & 0.01 & 0.00 & 1.00 & 1.00 & 0.00 & 0.09 & 0.02 & 0.11 & 0.03 \\
\hline $\mathrm{Ca}^{+2}$ & 0.64 & 0.63 & 0.42 & 0.01 & 0.60 & 0.00 & 0.63 & 0.51 & 0.01 & 0.71 & 0.02 & 0.61 & 0.67 \\
\hline $\mathrm{Cr}$ & 0.98 & 1.00 & 0.59 & 0.24 & 0.90 & 0.06 & 0.94 & 0.87 & 0.13 & 0.97 & 0.36 & 0.32 & 0.79 \\
\hline $\mathrm{CO}^{-2}$ & 0.97 & 0.97 & 0.63 & 0.14 & 0.82 & 0.02 & 0.98 & 0.94 & 0.12 & 0.92 & 0.26 & 0.86 & 0.84 \\
\hline $\mathrm{Cr}^{+3}$ & 0.99 & 1.00 & 0.59 & 0.61 & 0.99 & 0.00 & 0.33 & 0.15 & 0.10 & 0.95 & 0.87 & 0.92 & 0.45 \\
\hline $\mathrm{F}^{-}$ & 1.00 & 1.00 & 0.41 & 0.14 & 0.53 & 0.00 & 0.67 & 1.00 & 0.06 & 0.75 & 0.47 & 0.76 & 0.44 \\
\hline $\mathrm{Fe}^{+3}$ & 0.06 & 0.04 & 0.02 & 0.00 & 0.05 & 0.00 & 0.08 & 0.04 & 0.00 & 0.20 & 0.00 & 0.08 & 0.03 \\
\hline $\mathrm{K}^{-}$ & 1.00 & 1.00 & 0.02 & 1.00 & 1.00 & 0.00 & 1.00 & 1.00 & 0.00 & 0.00 & 0.00 & 1.00 & 0.91 \\
\hline $\mathrm{Mn}^{+4}$ & 0.01 & 0.01 & 0.00 & 0.00 & 0.00 & 0.00 & 0.01 & 0.01 & 0.00 & 0.00 & 0.00 & 0.01 & 0.99 \\
\hline $\mathrm{Na}^{+}$ & 0.96 & 0.96 & 0.56 & 0.25 & 0.87 & 0.01 & 0.96 & 0.92 & 0.11 & 0.95 & 0.44 & 0.92 & 0.85 \\
\hline $\mathrm{Ni}^{+2}$ & 0.04 & 0.04 & 0.01 & 0.00 & 0.02 & 0.00 & 0.03 & 0.02 & 0.00 & 0.05 & 0.00 & 0.03 & 0.32 \\
\hline $\mathrm{NO}^{-}$ & 1.00 & 1.00 & 0.79 & 0.73 & 1.00 & 0.06 & 0.98 & 1.00 & 0.17 & 0.99 & 0.95 & 0.99 & 0.97 \\
\hline NO3- & 0.99 & 1.00 & 0.71 & 0.49 & 0.96 & 0.01 & 0.94 & 0.89 & 0.17 & 0.99 & 0.66 & 0.98 & 0.93 \\
\hline $\mathrm{OH}^{\circ}$ & 0.94 & 0.90 & 0.06 & 0.07 & 0.74 & 0.00 & 0.68 & 0.50 & 0.01 & 0.82 & 0.16 & 0.78 & 0.55 \\
\hline $\mathrm{Pb}^{+2}$ & 1.00 & 1.00 & 1.00 & 1.00 & 1.00 & 0.00 & 1.00 & 1.00 & 0.00 & 0.00 & 0.00 & 1.00 & 0.06 \\
\hline $\mathrm{PO}^{-2}$ & 0.99 & 1.00 & 0.48 & 0.05 & 0.50 & 0.00 & 1.00 & 1.00 & 0.07 & 0.55 & 0.22 & 0.43 & 0.36 \\
\hline $\mathrm{Si}^{+4}$ & 0.00 & 0.00 & 0.04 & 0.00 & 0.09 & 0.00 & 0.00 & 0.00 & 0.01 & 0.00 & 0.15 & 0.00 & 0.03 \\
\hline $\mathrm{SO}_{4-2}$ & 0.90 & 0.79 & 0.83 & 0.13 & 0.55 & 0.00 & 0.99 & 0.98 & 0.44 & 0.95 & 0.21 & 0.73 & 0.67 \\
\hline $\mathrm{Sr}^{+2}$ & 0.00 & 0.00 & 0.00 & 0.00 & 0.00 & 0.00 & 0.00 & 0.00 & 0.00 & 0.00 & 0.00 & 0.00 & 0.00 \\
\hline $\mathrm{UO}_{2}+2$ & 0.59 & 0.39 & 0.04 & 0.03 & 0.35 & 0.00 & 0.38 & 0.36 & 0.01 & 0.73 & 0.07 & 0.05 & 0.23 \\
\hline $\mathrm{Zr}^{+4}$ & 1.00 & 1.00 & 0.73 & 0.10 & 0.96 & 0.00 & 0.06 & 1.00 & 0.15 & 0.64 & 0.32 & 0.67 & 0.21 \\
\hline TOC & 0.81 & 0.96 & 0.03 & 0.89 & 0.54 & 0.00 & 1.00 & 1.00 & 0.00 & 1.00 & 0.09 & 1.00 & 0.76 \\
\hline TOTAL & 0.95 & 0.94 & 0.54 & 0.27 & 0.88 & 0.00 & 0.91 & 0.84 & 0.10 & 0.96 & 0.47 & 0.91 & 0.83 \\
\hline
\end{tabular}


For use in the TWRS flowsheet, the resulting salt cake sludge partitions are further partitioned into soluble/insoluble fractions using mass weighted average water-washing solubility factors. Solubility factors for sludge, shown in Table 3-3, have been calculated for sludges for selected components through an analysis of data taken from 27 core samples (Colton 1995). For salt cake, a 99 wt\% solubility for all components was assumed. This assumption is supported by work done by W. W. Schulz (1978). Schulz dissolved salt cake taken from seven SSTs. The percentage of salt cake dissolved for these tanks is presented in Table 3-4. Normally, complete dissolution of the salts should occur. That complete dissolution does not occur is probably an indication of sludge particles embedded in the salt cake samples.

Solving these equations for $x$ gives the result $x=.187 \mathrm{~T}$, i.e., the amount of aluminum needed in the salt cake in order to make the overall solubility equal to $25 \mathrm{wt} \%$ is $18.7 \mathrm{wt} \%$ of the total aluminum. The HDW-MDL places $61.6 \mathrm{wt} \%$ of the aluminum in the salt cake. The ratio $18.7 / 61.6$, or .304 is, therefore, the relative salt cake reduction factor used to reassign aluminum from the salt cake to the sludge. In other words, the initial salt cake inventory is multiplied by 0.304 and the result becomes the new salt cake inventory and the balance is reassigned to the sludge. Similar treatment was performed on the other components with unreasonably high solubilities. These components were calcium, manganese, uranium, and zirconium.

The average sludge solubility for sodium from Colton (1995) is $76 \mathrm{wt} \%$. Applying the $76 \mathrm{wt} \%$ to the normalized HDW-MDL distribution of sodium in the sludge and $99 \mathrm{wt} \%$ to the salt cake combined with sodium already in the supernatant yields an overall $96 \mathrm{wt} \%$ solubility corresponding to $31 \mathrm{wt} \% \mathrm{Na}_{2} \mathrm{O}$ in the residual sludges on a waste oxide basis. Laboratory data, however, indicate that residual sludges after water washing have $\mathrm{Na}_{2} \mathrm{O}$ contents between 10 and 20 wt\% (Colton 1995). Therefore, the sludge and salt cake solubilities were adjusted to so that the residual $\mathrm{Na}_{2} \mathrm{O}$ composition for the composite waste stream was 14 percent corresponding to $99 \mathrm{wt} \%$ solubility for total sodium (includes sodium from cancrinite). ${ }^{1}$

High concentrations in the salt cake layers derived from the HDW-MDL for aluminum, calcium, manganese, nickel, uranium, and zirconium result in inordinately high solubilities that are inconsistent with the analytical data and known chemical behavior for these species. Analytical data on aluminum, for example, indicate that aluminum should not exceed $25 \mathrm{wt} \%$ soluble (LaFemina et al. 1995), and calcium, manganese, nickel, uranium, and zirconium are expected to be mostly insoluble, especially in an alkaline environment.

${ }^{1}$ Cancrinite, $2 \mathrm{NaAlSiO}_{4}: 0.52 \mathrm{NaNO}_{3}: 0.68 \mathrm{H}_{2} \mathrm{O}$, is an insoluble sodium aluminosilicate compound assumed by TWRS to represent all the insoluble sodium aluminosilicate compounds found in SSTs. There is an estimated 2.70E+03 MT of cancrinite in the TWRS inventory of which $4.44 \mathrm{E}+02 \mathrm{MT}$ is sodium. 


\section{WHC-SD-WM-TI-784 \\ Revision 0}

Table 3-3. Solubility Factors for Single-Shell Tanks

(Percent Soluble after Washing Waste in Water).

\begin{tabular}{|c|c|c|}
\hline Component & Sludge & Salt Cake \\
\hline $\mathrm{Ag}^{+}$ & 2.0 & 99 \\
\hline $\mathrm{Al}^{+3}$ & 8.0 & 99 \\
\hline $\mathrm{B}^{+3}$ & 59 & 99 \\
\hline $\mathrm{Ba}^{+2}$ & 0.2 & 99 \\
\hline $\mathrm{Bi}^{+3}$ & 0.7 & 99 \\
\hline $\mathrm{Ca}^{+2}$ & 3.0 & 99 \\
\hline $\mathrm{Cd}^{+2}$ & 11 & 99 \\
\hline $\mathrm{Co}^{+3}$ & 13 & 99 \\
\hline $\mathrm{Cr}^{+3}$ & 18 & 99 \\
\hline $\mathrm{Cu}^{+2}$ & 3.7 & 99 \\
\hline $\mathrm{Fe}^{+3}$ & 0.67 & 99 \\
\hline $\mathrm{K}^{+}$ & 64 & 99 \\
\hline $\mathrm{La}^{+3}$ & 0.24 & 99 \\
\hline $\mathrm{Mg}^{+2}$ & 1.7 & 99 \\
\hline $\mathrm{Mn}^{+4}$ & 0.92 & 99 \\
\hline $\mathrm{Na}^{+(\mathrm{a})}$ & 96 & 99 \\
\hline $\mathrm{Ni}^{+3}$ & 2.2 & 99 \\
\hline $\mathrm{Pb}^{+4}$ & 6.8 & 99 \\
\hline $\mathrm{PO}_{4}^{-3}$ & 40 & 99 \\
\hline $\mathrm{Si}^{+4}$ & 2.5 & 99 \\
\hline $\mathrm{Sr}^{+2}$ & 0.62 & 99 \\
\hline $\mathrm{UO}_{2}{ }^{+2}$ & 2.1 & 99 \\
\hline $\mathrm{Zn}^{+2}$ & 3.2 & 99 \\
\hline $\mathrm{Zr}^{+4}$ & 0.26 & 99 \\
\hline
\end{tabular}

aThe $\mathrm{Na}$ sludge wash factor for non-cancrinite $\mathrm{Na}$. was increased from $76 \mathrm{wt} \%$ to make the residual $\mathrm{Na}_{2} \mathrm{O}$ agree with experimental data. Typically, residual $\mathrm{Na}_{2} \mathrm{O}$ (including cancrinite) in washed sludges range from 10 to $20 \mathrm{wt} \% .14 \mathrm{wt} \%$ was chosen as it corresponds to 98 percent removal of all $\mathrm{Na}$, including the $\mathrm{Na}$ in cancrinite, from the sludge. 
WHC-SD-WM-TI-784

Revision 0

Table 3-4. Results of Salt Cake Dissolution Studies. ${ }^{a}$

\begin{tabular}{|c|c|}
\hline Tank & Percent Sltck Dissolved \\
\hline TX-116 & 98.3 \\
\hline B-105 & 99.0 \\
\hline S-105 & 99.8 \\
\hline S-109 & 98.8 \\
\hline S-110 & 100.0 \\
\hline SX-102 & 99.2 \\
\hline SX-103 & 99.7 \\
\hline Average & 99.3 \\
\hline
\end{tabular}

aTaken from RHO-SA-51, Removal of Radionuclides from Hanford Defense Waste Solutions, dated April 4, 1980.

This difficulty was resolved by reapportioning appropriate amounts of each component to the sludge while decreasing the amount in salt cake by an equal amount. Using aluminum as an example, the reapportioning was accomplished by solving the following simple system of equations:

$$
\begin{gathered}
x+y=\quad T \\
.99 x+.08 y=.25 T
\end{gathered}
$$

where $\quad \mathrm{x}=$ amount of aluminum in salt cake

$\mathrm{y}=$ amount of aluminum in sludge

$\mathrm{T}=$ total aluminum

The constants, .99 and .08 , are the assumed solubility factors taken from Table 3-3 and .25 is the desired overall solubility, so the second equation represents the portion of aluminum that is expected to be water soluble.

A charge balance was enforced on each tank primarily by adjusting hydroxide.

The final results are shown in Table 3-5. 


\section{WHC-SD-WM-TI-784}

Revision 0

Table 3-5. Single-Shell Tank Chemcal Inventory.

\begin{tabular}{|c|c|c|c|}
\hline Component & Soluble (MT) & Insoluble: (MT) & Total (MT) \\
\hline $\mathrm{Al}^{+3}$ & $7.39 \mathrm{E}+02$ & $2.65 \mathrm{E}+03$ & $2.96 \mathrm{E}+03$ \\
\hline $\mathrm{Bi}^{+3}$ & $9.58 \mathrm{E}+00$ & $2.22 \mathrm{E}+02$ & $2.62 E+02$ \\
\hline $\mathrm{Ca}^{+2}$ & $6.42 \mathrm{E}+00$ & $1.22 \mathrm{E}+02$ & $1.28 \mathrm{E}+02$ \\
\hline Cancrinite & $0.00 \mathrm{E}+00$ & $2.70 \mathrm{E}+03$ & $2.70 \mathrm{E}+03$ \\
\hline $\mathrm{Cl}^{-}$ & $4.07 \mathrm{E}+02$ & $7.63 \mathrm{E}+00$ & $4.15 E+02$ \\
\hline $\mathrm{CO}^{-2}$ & $1.62 \mathrm{E}+03$ & $3.86 \mathrm{E}+01$ & $1.66 \mathrm{E}+03$ \\
\hline $\mathrm{Cr}^{+3}$ & $1.47 \mathrm{E}+02$ & $1.21 E+02$ & $2.68 \mathrm{E}+02$ \\
\hline $\mathrm{F}^{-}$ & $7.86 \mathrm{E}+02$ & $2.62 \mathrm{E}+01$ & $8.12 E+02$ \\
\hline $\mathrm{Fe}^{+3}$ & $2.37 \mathrm{E}+01$ & $6.07 \mathrm{E}+02$ & $6.31 \mathrm{E}+02$ \\
\hline $\mathrm{K}^{+}$ & $5.29 \mathrm{E}+01$ & $2.43 \mathrm{E}+00$ & $5.53 \mathrm{E}+01$ \\
\hline $\mathrm{Mn}^{+4}$ & $2.41 E+00$ & $1.18 \mathrm{E}+02$ & $1.21 \mathrm{E}+02$ \\
\hline $\mathrm{Na}^{+}$ & $5.68 \mathrm{E}+04$ & $5.42 \mathrm{E}+02$ & $5.73 E+04$ \\
\hline $\mathrm{Ni}^{+3}$ & $4.06 \mathrm{E}+00$ & $1.99 \mathrm{E}+02$ & $2.03 E+02$ \\
\hline $\mathrm{NO}^{-}$ & $6.47 \mathrm{E}+03$ & $5.34 \mathrm{E}+01$ & $6.52 \mathrm{E}+03$ \\
\hline $\mathrm{NO}^{-}$ & $9.87 \mathrm{E}+04$ & $9.42 \mathbf{E}+02$ & $1.00 \mathrm{E}+05$ \\
\hline $\mathrm{OH}^{-}$ & $8.14 \mathrm{E}+03$ & $4.55 \mathrm{E}+03$ & $1.27 \mathrm{E}+04^{\mathrm{a}}$ \\
\hline $\mathrm{Pb}^{+4}$ & $3.29 \mathrm{E}+00$ & $2.50 \mathrm{E}+01$ & $2.83 \mathrm{E}+01$ \\
\hline $\mathrm{PO}^{3}$ & $2.90 \mathrm{E}+03$ & $1.83 E+03$ & $4.73 E+03$ \\
\hline $\mathrm{Si}^{+4}$ & $4.06 \mathrm{E}-01$ & $1.41 \mathrm{E}+01$ & $1.45 \mathrm{E}+01$ \\
\hline $\mathrm{SO}_{4-2}$ & $1.63 \mathrm{E}+03$ & $2.19 \mathrm{E}+01$ & $1.65 \mathrm{E}+03$ \\
\hline $\mathrm{Sr}^{+2}$ & $3.60 \mathrm{E}-01$ & $3.57 \mathrm{E}+01$ & $3.60 \mathrm{E}+01$ \\
\hline $\mathrm{UO}^{+2}$ & $9.67 \mathrm{E}+01$ & $1.50 \mathrm{E}+03$ & $1.60 \mathrm{E}+03$ \\
\hline $\mathrm{Zr}^{+4}$ & $3.82 \mathrm{E}+00$ & $3.78 E+02$ & $3.82 \mathrm{E}+02$ \\
\hline TOC & $4.58 \mathrm{E}+02$ & $1.51 \mathrm{E}+01$ & $4.73 E+02$ \\
\hline Total & $1.80 \mathrm{E}+05$ & $1.60 \mathrm{E}+04$ & $1.96 \mathrm{E}+05$ \\
\hline
\end{tabular}


WHC-SD-WM-TI-784

Revision 0

\subsection{RADIONUCLIDE INVENTORIES FOR SINGLE-SHELL TANKS}

The current TWRS inventory estimates are based on but not identical to the tank-by-tank distribution of radionuclides found in the TRAC database. The TRAC computer model (in combination with the RIBD computer code that calculates type and quantity of fission products) uses reactor fuel discharge records, process flowsheets, tank farm records, decay calculations, and solubility data to predict the various quantities of radionuclides that exited the reactors (with the exception of activation products) and the distribution of those radionuclides in the waste tanks. Activities of activation products $\left({ }^{14} \mathrm{C},{ }^{3} \mathrm{H}\right)$ were determined by hand calculations. The tank-by-tank estimates can be found in Appendix A (see Table A-3).

Data from TRAC formed the basis for radionuclides in the SSTs for many reports including the HDW-EIS, the TOR, and the TWRS-EIS. Adjustments were occasionally made in the distribution of radionuclides between DSTs and SSTs due mainly to new sample data for DSTs or different assumptions regarding the number of cesium and strontium capsules produced at the Waste Encapsulation Facility (WESF). However, the overall totals reported by TRAC were adhered to in these reports.

For the current TWRS inventory, the overall totals are the TRAC totals and the distribution between DSTs and SSTs follows closely that of the TWRS-EIS except for changes based on new sample data obtained for several DSTs.

Water solubility assumptions for radionuclides were taken from Table G1-1 of the TOR. These percent soluble factors are based on unpublished water-washing data taken from ten SSTs.

The cumulative results for the SSTs are shown in Table 3-6. The decay date for radionuclides is December 31, 1999. 
WHC-SD-WM-TI-784

Revision 0

Table 3-6. Single-Shell Tank Radionuclide Inventory. (decayed to December 31, 1999)

\begin{tabular}{|c|c|c|c|}
\hline Radionuclide & Soluble $(\mathrm{Ci})$ & Insoluble $(\mathrm{Ci})$ & Total $(\mathrm{Ci})$ \\
\hline${ }^{14} \mathrm{C}$ & $2.98 \mathrm{E}+03$ & $3.01 \mathrm{E}+01$ & $3.01 \mathrm{E}+03$ \\
\hline${ }^{90} \mathrm{Sr}^{\mathrm{a}}$ & $4.12 \mathrm{E}+05$ & $4.08 \mathrm{E}+07$ & $4.12 \mathrm{E}+07$ \\
\hline${ }^{99} \mathrm{Tc}$ & $8.89 \mathrm{E}+03$ & $8.89 \mathrm{E}+03$ & $1.78 \mathrm{E}+04$ \\
\hline${ }^{137} \mathrm{Cs}{ }^{\mathrm{a}}$ & $7.18 \mathrm{E}+06$ & $2.29 \mathrm{E}+06$ & $9.47 \mathrm{E}+06$ \\
\hline${ }^{237} \mathrm{~Np}$ & $6.95 \mathrm{E}+00$ & $6.25 \mathrm{E}+01$ & $6.94 \mathrm{E}+01$ \\
\hline${ }^{239} \mathrm{Pu}$ & $3.44 \mathrm{E}+02$ & $1.69 \mathrm{E}+04$ & $1.72 \mathrm{E}+04$ \\
\hline${ }^{240} \mathrm{Pu}$ & $9.15 \mathrm{E}+01$ & $4.18 \mathrm{E}+03$ & $4.27 \mathrm{E}+03$ \\
\hline${ }^{241} \mathrm{Pu}$ & $2.29 \mathrm{E}+03$ & $3.36 \mathrm{E}+04$ & $3.59 \mathrm{E}+04$ \\
\hline${ }^{241} \mathrm{Am}$ & $2.65 \mathrm{E}+03$ & $2.43 \mathrm{E}+04$ & $2.69 \mathrm{E}+04$ \\
\hline${ }^{244} \mathrm{Cm}$ & $1.20 \mathrm{E}+00$ & $1.19 \mathrm{E}+02$ & $1.20 \mathrm{E}+02$ \\
\hline Total & $7.61 \mathrm{E}+06$ & $4.32 \mathrm{E}-07$ & $5.08 \mathrm{E}+07$ \\
\hline
\end{tabular}

${ }^{a}$ Does not include daughter products. 
WHC-SD-WM-TJ-784

Revision 0

\subsection{DOUBLE-SHELL TANK INVENTORIES}

\subsection{CHEMICAL INVENTORIES FOR DOUBLE-SHELL TANKS}

\subsubsection{Background}

Estimates of DST compositions were derived primarily from sample data along with some independent studies that attempt to reconcile laboratory data with process knowledge and flowsheet records (Pajunen 1994, Schofield 1991).

There are uncharacterized solids in several DSTs. These tanks are 241-AN-104, 241-AN-106, 241-AW-102, and 241-AW-106. These solids may be sludges or precipitated salts. The bulk of the solids in these DSTs, however, are believed to be sodium salts that have precipitated from the concentrated liquid waste above since historical transaction records do not indicate that any wastes containing sludges were sent to any of these tanks. The salts assumed to be in these tanks are expected to dissolve upon retrieval. It was assumed that these solids have the same composition as the liquid above them and their volumes were added to the respective supernatant volumes.

The compositions and tank volumes used are representative of the period shortly before the beginning of the evaporator campaign in April 1994 when the DST inventory was relatively stable. Since that time, there have been additiors to the DST system from saltwell pumping, from facility cleanout operations, from transfers of liquid wastes between DSTs, and from concentration of dilute waste in the $242 \mathrm{~A}$ evaporator. All of these transactions have impacted the supernatant composition of a subset of the DSTs while the sludge has been largely unaffected.

\subsubsection{Water Solubility of the Bulk Inventory}

With the exception of tanks containing complexant concentrate (CC) waste, the amount of waste assumed to be soluble was determined from water-washing of sludge samples in the laboratory (Gray et al. 1993a and b, Herting 1994, Lumetla and Swanson 1993, Lumetta 1994, Peterson 1990). Values for the percent expected to be soluble are shown in Table 4-1. These values indicate the fraction of the total mass of a particular components expected to be soluble, i.e. these values include the amourit already present in the supernatant.

Because of a lack of data for CC wastes, the values used for this waste type are assumed factors based on knowledge of the chemical behavior of alkaline wastes with high organic content. Although sludge washing data for 242-SY-101, a CC tank, were released, there was some question whether the results could be applied to the other tanks as well since the relatively low amount of TRU in 242-SY-101 supernatant indicates less complexing 


\section{WHC-SD-WM-TI-784 \\ Revision 0}

ability than the other CC tanks. It was decided, therefore, to apply the data only to 242-SY-101 until sludge washing data for other CC wastes could be obtained.

The solubility factors for Neutralized Current Aging Waste (NCAW) in Table 4-1 are weighted averages of sludge washing data taken from botł 241-AZ-101 and 241-AZ-102, the only DSTs containing NCAW. However, sludge washing results vary so much between these tanks that wash factors specific to each tank would yield more accurate results. 
Table 4-1. Sludge Wash Factors for Double-Sihell Tank Waste Tanks, Assumed Percent Soluble in Water.

\begin{tabular}{|c|c|c|c|c|c|c|c|c|c|c|c|c|c|}
\hline & $\mathrm{CC}$ & NCAW & NCRW & PFP & SY-101 & SST & & $\mathrm{CC}$ & NCAW & NCRW & PFP & SY-101 & SST \\
\hline $\mathrm{Ag}+$ & $0 \%$ & $9 \%$ & $0 \%$ & $0 \%$ & $\mathrm{~N} / \mathrm{A}$ & $0 \%$ & $\mathrm{Ni} 2 \mathrm{Fe}(\mathrm{CN}) 6$ & $0 \%$ & $0 \%$ & $0 \%$ & $0 \%$ & $N / A$ & $0 \%$ \\
\hline $\mathrm{Al}+3$ & $95 \%$ & $48 \%$ & $9 \%$ & $2 \%$ & $97 \%$ & $25 \%$ & $\mathrm{NO} 2-$ & $99 \%$ & $97 \%$ & $81 \%$ & $99 \%$ & $99 \%$ & $99 \%$ \\
\hline $\mathrm{Am}+3$ & $95 \%$ & $2 \%$ & $0 \%$ & $0 \%$ & $19 \%$ & $10 \%$ & NO3- & $99 \%$ & $99 \%$ & $64 \%$ & $99 \%$ & $99 \%$ & $99 \%$ \\
\hline $\mathrm{As}+5$ & $0 \%$ & $36 \%$ & $0 \%$ & $0 \%$ & $\mathrm{~N} / \mathrm{A}$ & $0 \%$ & $\mathrm{~Np}+4$ & $0 \%$ & $15 \%$ & $0 \%$ & $0 \%$ & N/A & $10 \%$ \\
\hline$B+3$ & $95 \%$ & $27 \%$ & $0 \%$ & $0 \%$ & $95 \%$ & $0 \%$ & $\mathrm{OlI}-$ & $93 \%$ & $64 \%$ & $18 \%$ & $1 \%$ & $93 \%$ & $78 \%$ \\
\hline $\mathrm{Ba}+2$ & $90 \%$ & $3 \%$ & $0 \%$ & $0 \%$ & $90 \%$ & $0 \%$ & $\mathrm{~Pb}+4$ & $0 \%$ & $7 \%$ & $0 \%$ & $0 \%$ & $\mathrm{~N} / \mathrm{A}$ & $0 \%$ \\
\hline $\mathrm{Be}+2$ & $0 \%$ & $7 \%$ & $0 \%$ & $0 \%$ & $\mathrm{~N} / \mathrm{A}$ & $0 \%$ & PO4-3 & $99 \%$ & $92 \%$ & $0 \%$ & $60 \%$ & $99 \%$ & $50 \%$ \\
\hline $\mathrm{Bi}+3$ & $0 \%$ & $0 \%$ & $0 \%$ & $0 \%$ & N/A & $25 \%$ & $\begin{array}{c}1205: 24 W 02 \\
: 44 H 20 \\
\end{array}$ & $0 \%$ & $0 \%$ & $0 \%$ & $0 \%$ & $\mathrm{~N} / \mathrm{A}$ & $0 \%$ \\
\hline Cancrinite & $0 \%$ & $0 \%$ & $0 \%$ & $0 \%$ & $0 \%$ & $0 \%$ & $\mathrm{Pu}+4$ & $95 \%$ & $4 \%$ & $0 \%$ & $0 \%$ & $19 \%$ & $2 \%$ \\
\hline${ }^{14} \mathrm{C}$ & $0 \%$ & $95 \%$ & $20 \%$ & $82 \%$ & $0 \%$ & $99 \%$ & $\mathbf{R b}+$ & $0 \%$ & $96 \%$ & $0 \%$ & $0 \%$ & N/A & $0 \%$ \\
\hline $\mathrm{Ca}+2$ & $90 \%$ & $3 \%$ & $2 \%$ & $15 \%$ & $92 \%$ & $5 \%$ & $\mathrm{Re}+7$ & $0 \%$ & $11 \%$ & $0 \%$ & $0 \%$ & $\mathrm{~N} / \mathrm{A}$ & $0 \%$ \\
\hline $\mathrm{Cd}+2$ & $0 \%$ & $0 \%$ & $0 \%$ & $0 \%$ & $\mathrm{~N} / \mathrm{A}$ & $50 \%$ & $\mathrm{Rh}+3$ & $0 \%$ & $14 \%$ & $0 \%$ & $0 \%$ & $\mathrm{~N} / \mathrm{A}$ & $0 \%$ \\
\hline $\mathrm{Ce}+3$ & $0 \%$ & $6 \%$ & $0 \%$ & $0 \%$ & $\mathrm{~N} / \mathrm{A}$ & $1 \%$ & $\mathrm{Ru}+3$ & $0 \%$ & $9 \%$ & $0 \%$ & $0 \%$ & $N / A$ & $0 \%$ \\
\hline $\mathrm{Cl}$ & $99 \%$ & $96 \%$ & $97 \%$ & $95 \%$ & $99 \%$ & $95 \%$ & $\mathrm{Sb}+5$ & $0 \%$ & $3 \%$ & $0 \%$ & $0 \%$ & N/A & $0 \%$ \\
\hline $\mathrm{Cm}+3$ & $0 \%$ & $2 \%$ & $0 \%$ & $0 \%$ & $19 \%$ & $1 \%$ & $\mathrm{Se}+6$ & $0 \%$ & $39 \%$ & $0 \%$ & $0 \%$ & $\mathrm{~N} / \mathrm{A}$ & $0 \%$ \\
\hline $\mathrm{Co}+3$ & $0 \%$ & $6 \%$ & $0 \%$ & $0 \%$ & $\mathrm{~N} / \mathrm{A}$ & $0 \%$ & $\mathrm{Si}+4$ & $0 \%$ & $43 \%$ & $20 \%$ & $3 \%$ & $\mathrm{~N} / \mathrm{A}$ & $0 \%$ \\
\hline $\mathrm{CO} 3-2$ & $99 \%$ & $83 \%$ & $80 \%$ & $83 \%$ & $99 \%$ & $90 \%$ & $\mathrm{Sm}+3$ & $0 \%$ & $1 \%$ & $0 \%$ & $0 \%$ & N/A & $1 \%$ \\
\hline $\mathrm{Cr}+3$ & $95 \%$ & $91 \%$ & $2 \%$ & $48 \%$ & $5.3 \%$ & $10 \%$ & $\mathrm{Sn}+4$ & $0 \%$ & $0 \%$ & $0 \%$ & $0 \%$ & $\mathrm{~N} / \Lambda$ & $25 \%$ \\
\hline $\mathrm{Cs}+$ & $99 \%$ & $97 \%$ & $50 \%$ & $95 \%$ & $99 \%$ & $75 \%$ & $\mathrm{SO} 4-2$ & $99 \%$ & $99 \%$ & $44 \%$ & $31 \%$ & $99 \%$ & $98 \%$ \\
\hline $\mathrm{Cu}+2$ & $0 \%$ & S算 & $0 \%$ & $0 \%$ & $N / A$ & $0 \%$ & $\mathrm{Sr}+2$ & $95 \%$ & $1 \%$ & $0 \%$ & $0 \%$ & $57 \%$ & $1 \%$ \\
\hline$F_{-}$ & $99 \%$ & $97 \%$ & $93 \%$ & $91 \%$ & $99 \%$ & $95 \%$ & ToO4- & $0 \%$ & $81 \%$ & $57 \%$ & $99 \%$ & $100 \%^{a}$ & $50 \%$ \\
\hline $\mathrm{Fe}+3$ & $95 \%$ & $0 \%$ & $0 \%$ & $0 \%$ & $6.5 \%$ & $1 \%$ & $\mathrm{Te}+6$ & $0 \%$ & $8 \%$ & $0 \%$ & $0 \%$ & $\mathrm{~N} / \mathrm{A}$ & $0 \%$ \\
\hline $\mathrm{Ifg}+2$ & $0 \%$ & $0 \%$ & $0 \%$ & $0 \%$ & $\mathrm{~N} / \mathrm{A}$ & $99 \%$ & $\mathrm{~T} \mathrm{n}+4$ & $0 \%$ & $5 \%$ & $0 \%$ & $0 \%$ & $\mathrm{~N} / \mathrm{\Lambda}$ & $1 \%$ \\
\hline I- & $0 \%$ & $47 \%$ & $20 \%$ & $99 \%$ & $\mathrm{~N} / \mathrm{A}$ & $99 \%$ & $T i+4$ & $0 \%$ & $3 \%$ & $0 \%$ & $0 \%$ & $\mathrm{~N} / \mathrm{A}$ & $0 \%$ \\
\hline $\mathrm{K}+$ & $99 \%$ & $93 \%$ & $78 \%$ & $99 \%$ & $99 \%$ & $0 \%$ & $\mathrm{~T}+3$ & $0 \%$ & $6 \%$ & $0 \%$ & $0 \%$ & $\mathrm{~N} / \mathrm{A}$ & $0 \%$ \\
\hline $\mathrm{La}+3$ & $90 \%$ & $1 \%$ & $0 \%$ & $0 \%$ & $\mathrm{~N} / \mathrm{A}$ & $1 \%$ & $\mathrm{UO} 2+2$ & $95 \%$ & $61 \%$ & $0 \%$ & $52 \%$ & $95 \%$ & $5 \%$ \\
\hline $\mathrm{Lit}$ & $0 \%$ & $8 \%$ & $0 \%$ & $65 \%$ & $\mathrm{~N} / \mathrm{A}$ & $0 \%$ & $v+5$ & $0 \%$ & $36 \%$ & $0 \%$ & $0 \%$ & $\mathrm{~N} / \mathrm{A}$ & $0 \%$ \\
\hline $\mathrm{Mg}+2$ & $90 \%$ & $2 \%$ & $0 \%$ & $0 \%$ & $\mathrm{~N} / \mathrm{A}$ & $0 \%$ & $w+6$ & $0 \%$ & $0 \%$ & $0 \%$ & $0 \%$ & N/A & $0 \%$ \\
\hline $\mathrm{MnO} 2$ & $95 \%$ & $0 \%$ & $0 \%$ & $0 \%$ & $90 \%$ & $5 \%$ & $\mathrm{Zn}+2$ & $90 \%$ & $21 \%$ & $0 \%$ & $0 \%$ & $90 \%$ & $0 \%$ \\
\hline $\mathrm{Mo}+6$ & $95 \%$ & $94 \%$ & $0 \%$ & $0 \%$ & $95 \%$ & $0 \%$ & $7 r+4$ & $0 \%$ & $0 \%$ & $0 \%$ & $0 \%$ & $\mathrm{~N} / \mathrm{A}$ & $0 \%$ \\
\hline $\mathrm{Na}+$ & $99 \%$ & $97 \%$ & $72 \%$ & $91 \%$ & $99 \%$ & $99 \%$ & $\mathrm{ZrO} 2: 2 \mathrm{H} 2 \mathrm{O}$ & $90 \%$ & $0 \%$ & $0 \%$ & $0 \%$ & $\mathrm{~N} / \mathrm{A}$ & $5 \%$ \\
\hline $\mathrm{Nb}+5$ & $0 \%$ & $0 \%$ & $0 \%$ & $0 \%$ & N/A & $0 \%$ & TOC & $99 \%$ & $4.2 \%$ & $50 \%$ & $21 \%$ & $99 \%$ & $90 \%$ \\
\hline $\mathrm{Ni}+3$ & $95 \%$ & $0 \%$ & $0 \%$ & $0 \%$ & $15 \%$ & $0 \%$ & & & & & & & \\
\hline
\end{tabular}


The resulting soluble/insoluble split for the summation of the DST inventories are shown in Table 4-2.

Table 4-2. Double-Shell Tank Chemical Inventory.

\begin{tabular}{|l|c|c|c|}
\hline \multicolumn{1}{|c|}{ Component } & Soluble (MT) & Insoluble (MT) & Total (MT) \\
\hline $\mathrm{Al}^{+3}$ & $1.24 \mathrm{E}+03$ & $3.32 \mathrm{E}+02$ & $1.57 \mathrm{E}+03$ \\
\hline $\mathrm{Bi}^{+3}$ & $2.23 \mathrm{E}+00$ & $0.00 \mathrm{E}+00$ & $2.23 \mathrm{E}+00$ \\
\hline $\mathrm{Ca}^{+2}$ & $1.01 \mathrm{E}+01$ & $1.56 \mathrm{E}+01$ & $2.57 \mathrm{E}+01$ \\
\hline $\mathrm{Cl}^{-}$ & $2.71 \mathrm{E}+02$ & $1.37 \mathrm{E}+00$ & $2.72 \mathrm{E}+02$ \\
\hline $\mathrm{CO}^{-2}$ & $1.48 \mathrm{E}+03$ & $7.04 \mathrm{E}+01$ & $1.55 \mathrm{E}+03$ \\
\hline $\mathrm{Cr}^{+3}$ & $4.63 \mathrm{E}+01$ & $3.86 \mathrm{E}+01$ & $8.49 \mathrm{E}+01$ \\
\hline $\mathrm{F}^{-}$ & $3.47 \mathrm{E}+02$ & $4.20 \mathrm{E}+01$ & $3.89 \mathrm{E}+02$ \\
\hline $\mathrm{Fe}^{+3}$ & $8.08 \mathrm{E}+00$ & $1.54 \mathrm{E}+02$ & $1.62 \mathrm{E}+02$ \\
\hline $\mathrm{K}^{+}$ & $5.37 \mathrm{E}+02$ & $2.72 \mathrm{E}+01$ & $5.64 \mathrm{E}+02$ \\
\hline $\mathrm{Mn}^{+4}$ & $7.69 \mathrm{E}+00$ & $2.06 \mathrm{E}+01$ & $2.83 \mathrm{E}+01$ \\
\hline $\mathrm{Na}^{+}$ & $1.10 \mathrm{E}+04$ & $2.35 \mathrm{E}+02$ & $1.12 \mathrm{E}+04$ \\
\hline $\mathrm{Ni}^{+3}$ & $4.07 \mathrm{E}+00$ & $6.87 \mathrm{E}+00$ & $1.09 \mathrm{E}+01$ \\
\hline $\mathrm{NO}^{-}$ & $3.00 \mathrm{E}+03$ & $8.40 \mathrm{E}+00$ & $3.01 \mathrm{E}+03$ \\
\hline $\mathrm{NO}^{-}$ & $7.45 \mathrm{E}+03$ & $4.30 \mathrm{E}+01$ & $7.49 \mathrm{E}+03$ \\
\hline $\mathrm{OH}^{-}$ & $2.92 \mathrm{E}+03$ & $1.09 \mathrm{E}+03$ & $4.01 \mathrm{E}+03$ \\
\hline $\mathrm{Pb}^{+4}$ & $1.89 \mathrm{E}+00$ & $4.31 \mathrm{E}+00$ & $6.20 \mathrm{E}+00$ \\
\hline $\mathrm{PO}^{-3}$ & $2.13 \mathrm{E}+02$ & $2.14 \mathrm{E}+01$ & $2.37 \mathrm{E}+02$ \\
\hline $\mathrm{Si}^{+4}$ & $1.55 \mathrm{E}+01$ & $2.18 \mathrm{E}+02$ & $2.34 \mathrm{E}+02$ \\
\hline $\mathrm{SO}^{-2}$ & $3.90 \mathrm{E}+02$ & $3.61 \mathrm{E}+00$ & $3.94 \mathrm{E}+02$ \\
\hline $\mathrm{Si}^{+2}$ & $1.86 \mathrm{E}-02$ & $6.00 \mathrm{E}-01$ & $6.19 \mathrm{E}-01$ \\
\hline $\mathrm{UO}^{+2}$ & $9.25 \mathrm{E}+00$ & $3.36 \mathrm{E}+01$ & $4.28 \mathrm{E}+01$ \\
\hline $\mathrm{Zr}^{+4}$ & $4.90 \mathrm{E}-01$ & $3.05 \mathrm{E}+02$ & $3.05 \mathrm{E}+02$ \\
\hline $\mathrm{TOC}^{-4}+97 \mathrm{E}+02$ & $7.21 \mathrm{E}+01$ & $6.69 \mathrm{E}+02$ \\
\hline \hline $\mathrm{Total}^{+3}$ & $2.96 \mathrm{E}+04$ & $2.74 \mathrm{E}+03$ & $3.23 \mathrm{E}+04$ \\
\hline
\end{tabular}




\subsection{RADIONUCLIDE INVENTORIES FOR DOUBLE-SHELL TANKS}

Radionuclide inventories for each DST are shown in Table A-4. The decay date is December 31, 1999. DST radionuclide estimates are again based primarily on sample data. Some adjustments (normalization) were necessary to maintain consistency with the Integrated Data Base (IDB). The IDB, maintained at the Oak Ridge National Laboratories (ORNL), is a compilation of historic data on inventories and character stics of commercial and government spent nuclear fuel and radioactive wastes. The cumulative activities for SSTs, DSTs, and capsules reported in the IDB should agree with the TRAC output with most of the adjustments mentioned in Section 2.1 .

The values for the Hanford Site DSTs shown in the JDB are based on an earlier set of DST sample data and assumptions. The estimates obtained from the more recent set of DST data used in this study compare well with the IDB values. A comparison by waste type is shown in Table 4-3. The large disparities between the ${ }^{14} \mathrm{C}$ numbers are caused by a conservative assumption in TRAC that ${ }^{14} \mathrm{C}$ losses were $90 \mathrm{wt} \%$ when it is more likely that ${ }^{14} \mathrm{C}$ were closer to $100 \mathrm{wt} \%$.

The normalized (IDB) values were adopted for the current TWRS inventory. To do this, radionuclides in each DST was adjusted by waste type proportionately so that the totals by waste type were the same as those shown in the "IDB" column of Table 4-3. The decay date is December 31, 1999.

${ }^{99} \mathrm{Tc}$ concentrations were not reported by the laboratory for several DSTs. In that event, the technetium inventories were estimated by distributing the remaining total given in the IDB among the remaining DSTs in direct proportion to the sodium concentration for a given tank.

In the case of the NCAW, NCRW, and PFP tanks (241-AZ-101, 241-AZ-102, 241-AW-103, 241-AW-105, 241-SY-102), the plutonium and americium values in the IDB are based on "tracking data," i.e. a large number of samples of homogeneous batches of waste taken at PUREX prior to transfer to tank farms (Pajunen 1994). 


\begin{tabular}{|c|c|c|c|c|c|c|c|c|c|c|c|c|}
\hline \multirow{2}{*}{$\begin{array}{l}\text { Radio- } \\
\text { nuclide }\end{array}$} & \multicolumn{2}{|c|}{$\mathrm{CC}$} & \multicolumn{2}{|c|}{ DSSF } & \multicolumn{2}{|c|}{ NCAW } & \multicolumn{2}{|c|}{ NCRW } & \multicolumn{2}{|c|}{ PFP } & \multicolumn{2}{|c|}{ TOTAL } \\
\hline & $\begin{array}{l}\text { Sample } \\
\text { (Ci) }\end{array}$ & $\begin{array}{r}\text { IDB } \\
\text { (Ci) } \\
\end{array}$ & $\begin{array}{l}\text { Sample } \\
\text { (Ci) }\end{array}$ & $\begin{array}{l}\text { IDB } \\
\text { (Ci) } \\
\end{array}$ & $\begin{array}{c}\text { Sample } \\
\text { (Ci) }\end{array}$ & $\begin{array}{l}\text { IDB } \\
\text { (Ci) }\end{array}$ & $\begin{array}{l}\text { Sample } \\
\text { (Ci) }\end{array}$ & $\begin{array}{r}\text { IDB } \\
\text { (Ci) }\end{array}$ & $\begin{array}{l}\text { Sample } \\
\text { (Ci) }\end{array}$ & $\begin{array}{r}\text { IDB } \\
\text { (Ci) }\end{array}$ & $\begin{array}{l}\text { Sample } \\
\text { (Ci) }\end{array}$ & $\begin{array}{r}\text { IDB } \\
\text { (Ci) }\end{array}$ \\
\hline${ }^{14} \mathrm{C}$ & $7.15 \mathrm{E}+00$ & $1.98 \mathrm{E}+03$ & $1.31 \mathrm{E}+01$ & $1.29 \mathrm{E}+01$ & $1.04 \mathrm{E}+01$ & $3.50 \mathrm{E}+02$ & $0.00 \mathrm{E}+000$ & $0.00 \mathrm{E}+00$ & $1.19 \mathrm{E}+00$ & $0.00 \mathrm{E}+00$ & $3.18 \mathrm{E}+01$ & $2.34 \mathrm{E}+03$ \\
\hline${ }^{90} \mathrm{Sr}$ & $1.88 \mathrm{E}+06$ & $4.05 \mathrm{E}+06$ & $1.90 \mathrm{E}+06$ & $1.02 \mathrm{E}+05$ & $8.91 E+06$ & $8.46 \mathrm{E}+06$ & $1.04 \mathrm{E}+05$ & $5.11 \mathrm{E}+04$ & $5.25 \mathrm{E}+04$ & $5.25 \mathrm{E}+04$ & $1.59 \mathrm{E}+07$ & $1.24 \mathrm{E}+07$ \\
\hline${ }^{99} \mathrm{Tc}$ & $8.36 \mathrm{E}+03$ & $8.38 \mathrm{E}+03$ & $3.83 \mathrm{E}+03$ & $3.79 \mathrm{E}+03$ & $2.35 \mathrm{E}+03$ & $2.10 \mathrm{E}+03$ & $7.54 \mathrm{E}+01$ & $0.00 \mathrm{E}+00$ & $2.81 \mathrm{E}+01$ & $0.00 \mathrm{E}+00$ & $1.45 \mathrm{E}+04$ & $1.43 E+04$ \\
\hline${ }^{137} \mathrm{Cs}$ & $5.85 \mathrm{E}+06$ & $4.88 E+06$ & $1.10 \mathrm{E}+07$ & $1.04 \mathrm{E}+07$ & $1.01 \mathrm{E}+07$ & $9.80 \mathrm{E}+06$ & $5.49 \mathrm{E}+05$ & $3.04 \mathrm{E}+05$ & $6.75 \mathrm{E}+04$ & $6.75 \mathrm{E}+04$ & $2.76 \mathrm{E}+07$ & $2.54 \mathrm{E}+07$ \\
\hline${ }^{239} \mathrm{Pu}$ & $4.05 \mathrm{E}+03$ & $3.99 \mathrm{E}+03$ & $8.00 \mathrm{E}+02$ & $1.57 \mathrm{E}+02$ & $1.7 \mathrm{IE}+03$ & $1.75 \mathrm{E}+03$ & $1.42 \mathrm{E}+03$ & $1.22 \mathrm{E}+03$ & $4.44 \mathrm{E}+03$ & $2.08 \mathrm{E}+03$ & $1.24 \mathrm{E}+04$ & $9.20 \mathrm{E}+03$ \\
\hline${ }^{240} \mathrm{Pu}$ & $1.01 \mathrm{E}+03$ & $1.00 \mathrm{E}+03$ & $2.56 \mathrm{E}+01$ & $2.41 \mathrm{E}+01$ & $4.83 \mathrm{E}+02$ & $3.21 \mathrm{E}+02$ & $4.02 \mathrm{E}+02$ & $3.44 \mathrm{E}+02$ & $1.25 \mathrm{E}+03$ & $7.49 \mathrm{E}+02$ & $3.17 \mathrm{E}+03$ & $2.44 \mathrm{E}+03$ \\
\hline${ }^{241} \mathrm{Pu}$ & $1.06 \mathrm{E}+03$ & $1.03 \mathrm{E}+03$ & $4.30 \mathrm{E}+01$ & $7.11 \mathrm{E}+01$ & $1.44 \mathrm{E}+04$ & $9.34 \mathrm{E}+03$ & $1.20 \mathrm{E}+04$ & $1.02 E+04$ & $2.23 E+04$ & $1.84 \mathrm{E}+04$ & $4.98 \mathrm{E}+04$ & $3.91 \mathrm{E}+04$ \\
\hline${ }^{241} \mathrm{Am}$ & $1.88 \mathrm{E}+04$ & $1.68 \mathrm{E}+04$ & $3.39 \mathrm{E}+03$ & $1.68 \mathrm{E}+02$ & $7.05 \mathrm{E}+04$ & $4.89 \mathrm{E}+04$ & $6.72 \mathrm{E}+02$ & $3.49 \mathrm{E}+02$ & $3.46 \mathrm{E}+04$ & $1.13 \mathrm{E}+04$ & $1.28 \mathrm{E}+05$ & $7.76 \mathrm{E}+04$ \\
\hline Total & $7.76 \mathrm{E}+06$ & $8.95 \mathrm{E}+06$ & $1.29 \mathrm{E}+07$ & $1.05 \mathrm{E}+07$ & $1.91 \mathrm{E}+07$ & $1.83 \mathrm{E}+07$ & $6.68 \mathrm{E}+05$ & $3.67 \mathrm{E}+05$ & $1.83 \mathrm{E}+05$ & $1.53 \mathrm{E}+05$ & $4.37 \mathrm{E}+07$ & $3.55 E+07$ \\
\hline
\end{tabular}




\section{WHC-SD-WM-TI-784}

Revision 0

The cumulative results for the DSTs are shown in Table 4-4.

Table 4-4. Double-Shell Tank Radionuclide Inventory. ${ }^{a}$ (decayed to December 31, 1999)

\begin{tabular}{|c|c|c|c|}
\hline Radionuclide & Soluble $(\mathrm{Ci})$ & Insoluble $(\mathrm{Ci})$ & Total $(\mathrm{Ci})$ \\
\hline${ }^{14} \mathrm{C}$ & $3.46 \mathrm{E}+02$ & $1.99 \mathrm{E}+03$ & $2.34 \mathrm{E}+04$ \\
\hline${ }^{90} \mathrm{Sr}$ & $1.47 \mathrm{E}+06$ & $1.09 \mathrm{E}+07$ & $1.24 \mathrm{E}+07$ \\
\hline${ }^{99} \mathrm{Tc}$ & $1.39 \mathrm{E}+04$ & $4.04 \mathrm{E}+02$ & $1.43 \mathrm{E}+04$ \\
\hline${ }^{137} \mathrm{Cs}$ & $2.47 \mathrm{E}+07$ & $7.16 \mathrm{E}+05$ & $2.54 \mathrm{E}+07$ \\
\hline${ }^{237} \mathrm{~Np}$ & $0.00 \mathrm{E}+00$ & $0.00 \mathrm{E}+00$ & $0.00 \mathrm{E}+00$ \\
\hline${ }^{239} \mathrm{Pu}$ & $1.31 \mathrm{E}+03$ & $7.89 \mathrm{E}+03$ & $9.20 \mathrm{E}+03$ \\
\hline${ }^{240} \mathrm{Pu}$ & $3.39 \mathrm{E}+02$ & $2.10 \mathrm{E}+03$ & $2.44 \mathrm{E}+03$ \\
\hline${ }^{241} \mathrm{Pu}$ & $2.50 \mathrm{E}+03$ & $3.66 \mathrm{E}+04$ & $3.91 \mathrm{E}+04$ \\
\hline${ }^{241} \mathrm{Am}$ & $5.31 \mathrm{E}+03$ & $7.23 \mathrm{E}+04$ & $7.76 \mathrm{E}+04$ \\
\hline${ }^{244} \mathrm{Cm}$ & $0.00 \mathrm{E}+00$ & $0.00 \mathrm{E}+00$ & $0.00 \mathrm{E}+00$ \\
\hline${ }^{T} \mathrm{Total}$ & $2.62 \mathrm{E}+07$ & $1.17 \mathrm{E}+07$ & $3.79 \mathrm{E}+07$ \\
\hline
\end{tabular}

"Does not include daughter products. 
WHC-SD-WM-TI-784

Revision 0

This page intentionally left blank. 
WHC-SD-WM-TI-784

Revision 0

\subsection{CONTRIBUTIONS FROM FUTURE FA.CILITY WASTES}

The Operational Waste Volume Projection (OWVP) document (Strode 1994) for FY 1994 reported that approximately $1.2 \mathrm{E}+07 \mathrm{~L}$ of waste from routine facility operations and facility decommissioning activities would be added to the: DST system. Additionally, interim stabilization of SSTs would result in the transfer of $1.55 \mathrm{E}+07 \mathrm{~L}$ of saltwell liquids from the SSTs to the DSTs.

To account for these assumptions, compositions of the miscellaneous waste streams from the various facilities/areas listed in the OWVP were obtained from an earlier waste volume projection document (Strode 1988). Subsequent OWVP documents have not included composition data. Average saltwell liquid composition was taken from (RHO 1985).

Table 5-1 shows the contribution to DSTs from facility operations and transfer of saltwell liquid from the SSTs. Saltwell liquid transfers do not result in a net gain to the underground tanks except for small volumes of flush water. Radionuclide data for facilities waste and saltwell liquids are not included due to a lack of reliable information. 
WHC-SD-WM-TI-784

Revision 0

Table 5-1. Waste Contributions from Facilities.

\begin{tabular}{|c|c|c|c|}
\hline Component & Facilities (MT) & Saltwell Liquid & Total \\
\hline $\mathrm{Al}^{+3}$ & $7.56 \mathrm{E}+01$ & $1.52 \mathrm{E}+02$ & $2.28 \mathrm{E}+02$ \\
\hline $\mathrm{Ca}^{+2}$ & $7.17 \mathrm{E}+00$ & & $7.17 \mathrm{E}+00$ \\
\hline $\mathrm{Cd}^{+2}$ & $7.46 \mathrm{E}+00$ & & $7.46 \mathrm{E}+00$ \\
\hline $\mathrm{Cl}^{-}$ & $3.61 \mathrm{E}-03$ & & $3.61 \mathrm{E}-03$ \\
\hline $\mathrm{Co}^{+3}$ & $4.08 \mathrm{E}-02$ & & $4.08 \mathrm{E}-02$ \\
\hline $\mathrm{CO}_{3}{ }^{-2}$ & & $4.70 \mathrm{E}+01$ & $4.70 \mathrm{E}+01$ \\
\hline $\mathrm{Cr}^{+3}$ & & $1.17 \mathrm{E}+01$ & $1.17 \mathrm{E}+01$ \\
\hline $\mathrm{F}^{-}$ & $4.09 \mathrm{E}+00$ & $6.38 \mathrm{E}+00$ & $1.05 \mathrm{E}+01$ \\
\hline $\mathrm{Fe}^{+3}$ & $4.51 \mathrm{E}+00$ & & $4.51 \mathrm{E}+00$ \\
\hline $\mathrm{H}_{2} \mathrm{O}$ & $1.46 \mathrm{E}+04$ & $1.25 \mathrm{E}+0<$ & $1.71 \mathrm{E}+004$ \\
\hline $\mathrm{K}^{+}$ & $1.17 \mathrm{E}+02$ & & $1.17 \mathrm{E}+02$ \\
\hline $\mathrm{Mg}^{+2}$ & $7.38 \mathrm{E}+00$ & & $7.38 \mathrm{E}+00$ \\
\hline $\mathrm{Mn}^{+4}$ & $4.09 \mathrm{E}+01$ & & $4.09 \mathrm{E}+01$ \\
\hline $\mathrm{Na}^{+}$ & $2.81 \mathrm{E}+02$ & $3.31 \mathrm{E}+03$ & $3.59 \mathrm{E}+03$ \\
\hline $\mathrm{Ni}^{+3}$ & $7.00 \mathrm{E}-02$ & & $7.00 \mathrm{E}-02$ \\
\hline $\mathrm{NO}_{2}^{-}$ & $1.16 \mathrm{E}+01$ & $1.48 \mathrm{E}+03$ & $1.49 \mathrm{E}+03$ \\
\hline $\mathrm{NO}_{3}^{-}$ & $4.12 \mathrm{E}+02$ & $2.04 \mathrm{E}+03$ & $2.45 \mathrm{E}+03$ \\
\hline $\mathrm{OH}^{-}$ & $7.73 \mathrm{E}-01$ & $3.67 \mathrm{E}+02$ & $3.68 \mathrm{E}+02$ \\
\hline $\mathrm{Pb}^{+4}$ & $4.43 \mathrm{E}+00$ & & $4.43 \mathrm{E}+00$ \\
\hline $\mathrm{PO}_{4}^{-3}$ & $8.60 \mathrm{E}+01$ & $9.70 \mathrm{E}+01$ & $1.83 \mathrm{E}+02$ \\
\hline $\mathrm{Si}^{+4}$ & $3.19 \mathrm{E}-03$ & & $3.19 \mathrm{E}-03$ \\
\hline $\mathrm{SO}_{4}{ }^{-2}$ & $1.30 \mathrm{E}+00$ & & $1.30 \mathrm{E}+00$ \\
\hline $\mathrm{TOC}^{+3}$ & $1.81 \mathrm{E}+00$ & $2.16 \mathrm{E}+02$ & $2.18 \mathrm{E}+02$ \\
\hline $\mathrm{UO}_{2}{ }^{+2}$ & $1.44 \mathrm{E}-02$ & & $1.44 \mathrm{E}-02$ \\
\hline $\mathrm{Total}^{-3}$ & $1.57 \mathrm{E}+04$ & $2.07 \mathrm{E}+04$ & $3.64 \mathrm{E}+04$ \\
\hline & & & \\
\hline & & & \\
\hline
\end{tabular}


WHC-SD-WM-TI-784

Revision 0

\subsection{TOTAL INVENTORY FOR TANK WASTE REMEDIATION SYSTEM PROCESS FLOWSHEET}

Tables A-1 through A-4 in Appendix A shows the chernical and radionuclide inventory estimates for single-shell and double-shell tanks recommended for use in the TWRS

flowsheet. The amount of water shown for each tank is not the amount estimated to be in the tank presently but rather is the amount needed to make the soluble portion $5 M \mathrm{Na}$ or the insoluble portion $10 \mathrm{wt} \%$ solids, whichever is greater. This was the retrieval assumption used for the TWRS flowsheet. Also shown is the transfer of approximately $1.55 \mathrm{E}+07 \mathrm{~L}$ of saltwell liquid from SSTs to DSTs.

Consistency with previous estimates, i.e., the HDW-EIS, the TWRS-EIS, and the IDB has been largely maintained. Table 6-1, the sum of Tables 3-5, 4-2, and column two of Table 5-1 for chemicals, summarizes total single-shell and double-shell chemical inventories after facility wastes have been added and saltwell pumping has been completed. Table 6-2, the sum of Tables 3-6 and 4-3, does the same for radionuclicles.

Reconciliation of previous estimates with the new estimates produced by LANL will no doubt result in changes to Table 6-1. Some of these changes may have a large impact on facilities design, retrieval schedules, and other design considerations. 
Table 6-1. Chemical Inventory for Tank Waste Remediation Systems Reference Feed.

\begin{tabular}{|c|c|c|c|c|c|c|}
\hline \multirow{2}{*}{ Component } & \multicolumn{3}{|c|}{ Soluble } & \multicolumn{3}{|c|}{ Insoluble } \\
\hline & Total (MT) & SST (MT) & $\operatorname{DST}(\mathrm{MT})$ & Total (MT) & SST (MT) & DST (MT) \\
\hline Total Mass Flow & $7.26 \mathrm{E}+05$ & $5.74 \mathrm{e}+05$ & $1.52 \mathrm{E}+05$ & $1.97 e+04$ & $1.68 \mathrm{e}+04$ & $2.88 \mathrm{e}+03$ \\
\hline $\mathrm{Ag}+$ & $3.20 \mathrm{E}-01$ & & $3.20 \mathrm{E}-01$ & $1.48 \mathrm{E}+00$ & $2.80 \mathrm{E}-07$ & $1.48 \mathrm{E}+00$ \\
\hline $\mathrm{Al}(\mathrm{OH}) 4$ & $6.99 \mathrm{E}+03$ & $2.07 \mathrm{E}+03$ & $4.93 \mathrm{E}+03$ & & & \\
\hline $\mathrm{T} \mathrm{Al}+3$ & & & & $2.31 \mathrm{E}+03$ & $2.20 \mathrm{E}+03$ & $1.11 \mathrm{E}+02$ \\
\hline $\mathrm{As}+5$ & 4.05E-05 & & $4.05 \mathrm{E}-05$ & $2.26 \mathrm{E}-03$ & & $2.26 \mathrm{E}-03$ \\
\hline $\mathrm{B}+3$ & $3.43 \mathrm{E}-04$ & & $3.43 \mathrm{E}-04$ & & & \\
\hline $\mathrm{Ba}+2$ & $7.91 \mathrm{E}-05$ & & $7.91 \mathrm{E}-01$ & $3.74 \mathrm{E}+00$ & $5.89 \mathrm{E}-01$ & $3.15 \mathrm{E}+00$ \\
\hline $\mathrm{Be}+2$ & 2.43E-05 & & $2.43 \mathrm{E}-05$ & $3.08 \mathrm{E}-03$ & & $3.08 \mathrm{E}-03$ \\
\hline $\mathrm{Bi}+3$ & $1.18 \mathrm{E}+01$ & $9.58 \mathrm{E}+00$ & $2.23 \mathrm{E}+00$ & $2.52 \mathrm{E}+02$ & $2.52 \mathrm{E}+02$ & \\
\hline $\mathrm{Ca}+2$ & $1.65 \mathrm{E}+01$ & $6.42 \mathrm{E}+00$ & $1.01 \mathrm{E}+01$ & $1.45 \mathrm{E}+02$ & $1.22 \mathrm{E}+02$ & $2.28 \mathrm{E}+01$ \\
\hline Cancrinite & & & & $2.70 \mathrm{E}+03$ & $2.70 \mathrm{E}+03$ & \\
\hline $\mathrm{Cd}+2$ & $7.63 \mathrm{E}+00$ & & $7.63 \mathrm{E}+00$ & $6.55 \mathrm{E}+00$ & & $6.55 \mathrm{E}+00$ \\
\hline $\mathrm{Ce}+3$ & $2.36 \mathrm{E}+00$ & $2.34 \mathrm{E}+00$ & $2.26 \mathrm{E}-04$ & $2.35 \mathrm{E}+02$ & $2.32 \mathrm{E}+02$ & $2.78 \mathrm{E}+00$ \\
\hline $\mathrm{Cl}-$ & $6.78 \mathrm{E}+02$ & $4.07 \mathrm{E}+02$ & $2.71 \mathrm{E}+02$ & $9.30 \mathrm{E}+00$ & $7.63 \mathrm{E}+00$ & $1.37 \mathrm{E}+00$ \\
\hline $\mathrm{CO} 3-2$ & $3.10 \mathrm{E}+03$ & $1.58 \mathrm{E}+03$ & $1.53 \mathrm{E}+03$ & $1.09 \mathrm{E}+02$ & $3.86 \mathrm{E}+01$ & $7.04 \mathrm{E}+01$ \\
\hline $\mathrm{Cr}(\mathrm{OH}) 4-$ & $4.46 \mathrm{E}+02$ & $3.13 E+02$ & $1.33 \mathrm{E}+02$ & & & \\
\hline $\mathrm{Cr}+3$ & & & & $1.60 \mathrm{E}+02$ & $1.21 \mathrm{E}+02$ & $3.87 \mathrm{E}+01$ \\
\hline $\mathrm{Cs}+$ & $1.88 \mathrm{E}+00$ & $5.06 \mathrm{E}-01$ & $1.37 \mathrm{E}+00$ & $2.06 \mathrm{E}-01$ & $1.76 \mathrm{E}-01$ & $3.05 \mathrm{E}-02$ \\
\hline $\mathrm{Cu}+2$ & $9.89 \mathrm{E}-04$ & & $9.89 \mathrm{E}-04$ & $1.88 \mathrm{E}-01$ & & $1.88 \mathrm{E}-01$ \\
\hline F- & $1.14 \mathrm{E}+03$ & $7.80 \mathrm{E}+02$ & $3.57 \mathrm{E}+02$ & $6.82 \mathrm{E}+01$ & $2.62 \mathrm{E}+01$ & $4.20 \mathrm{E}+01$ \\
\hline $\mathrm{Fe}+3$ & $3.44 \mathrm{E}+01$ & $2.37 \mathrm{E}+01$ & $1.07 \mathrm{E}+01$ & $7.62 \mathrm{E}+02$ & $6.07 \mathrm{E}+02$ & $1.56 \mathrm{E}+02$ \\
\hline $\mathrm{H} 2 \mathrm{O}$ & $5.08 \mathrm{E}+05$ & $3.99 \mathrm{E}+05$ & $1.09 \mathrm{E}+05$ & & & \\
\hline $\mathrm{Hg}+2$ & $5.84 \mathrm{E}-04$ & & $5.84 \mathrm{E}-04$ & $8.81 \mathrm{E}-02$ & & $8.81 \mathrm{E}-02$ \\
\hline $\mathrm{K}+$ & $7.07 \mathrm{E}+02$ & $5.29 \mathrm{E}+01$ & $6.54 \mathrm{E}+02$ & $2.96 \mathrm{E}+01$ & $2.42 \mathrm{E}+00$ & $2.72 \mathrm{E}+01$ \\
\hline $\mathrm{La}+3$ & $2.39 \mathrm{E}-05$ & $1.88 \mathrm{E}-02$ & $2.20 \mathrm{E}-05$ & $2.30 \mathrm{E}+01$ & $1.86 \mathrm{E}+00$ & $2.11 \mathrm{E}+01$ \\
\hline $\mathrm{Mg}+2$ & $1.46 \mathrm{E}-04$ & & $1.46 \mathrm{E}-04$ & $8.25 \mathrm{E}+00$ & & $8.25 \mathrm{E}+00$ \\
\hline $\mathrm{Mn}+4$ & $1.08 \mathrm{E}+01$ & $2.42 \mathrm{E}+00$ & $8.39 \mathrm{E}+00$ & $1.80 \mathrm{E}+02$ & $1.18 \mathrm{E}+02$ & $6.15 \mathrm{E}+01$ \\
\hline $\mathrm{Mo}+6$ & $7.23 \mathrm{E}-03$ & & $7.23 \mathrm{E}-03$ & $3.21 \mathrm{E}-02$ & & $3.21 \mathrm{E}-02$ \\
\hline
\end{tabular}


Table 6-1. Chemical Inventory for Tank Waste Remediation Systems Reference Feed.

\begin{tabular}{|c|c|c|c|c|c|c|}
\hline \multirow{2}{*}{ Component } & \multicolumn{3}{|c|}{ Soluble } & \multicolumn{3}{|c|}{ Insoluble } \\
\hline & Total (MT) & $\mathrm{SST}(\mathrm{MT})$ & DST (MT) & Total (MT) & SST (MT) & DST (MT) \\
\hline $\mathrm{Na}+$ & $6.80 \mathrm{E}+04$ & $5.34 \mathrm{E}+04$ & $1.46 \mathrm{E}+04$ & $7.77 \mathrm{E}+02$ & $5.42 \mathrm{E}+02$ & $2.35 \mathrm{E}+02$ \\
\hline $\mathrm{Ni}+3$ & $8.21 \mathrm{E}+00$ & $4.07 \mathrm{E}+00$ & $4.14 \mathrm{E}+00$ & $2.06 \mathrm{E}+02$ & $1.99 \mathrm{E}+02$ & $6.87 \mathrm{E}+00$ \\
\hline NO2- & $9.47 \mathrm{E}+03$ & $4.98 \mathrm{E}+03$ & $4.49 \mathrm{E}+03$ & $6.18 \mathrm{E}+01$ & $5.34 \mathrm{E}+01$ & $8.40 \mathrm{E}+00$ \\
\hline NO3- & $1.07 \mathrm{E}+05$ & $9.66 E+04$ & $9.90 \mathrm{E}+03$ & $9.85 \mathrm{E}+02$ & $9.42 \mathrm{E}+02$ & $4.31 \mathrm{E}+01$ \\
\hline $\mathrm{OH}-$ & $1.43 \mathrm{E}+04$ & $9.25 \mathrm{E}+03$ & $5.08 \mathrm{E}+03$ & $5.63 \mathrm{E}+03$ & $4.54 \mathrm{E}+03$ & $1.09 \mathrm{E}+03$ \\
\hline $\mathrm{Pb}+4$ & $9.60 \mathrm{E}+00$ & $3.29 \mathrm{E}+00$ & $6.31 E+00$ & $2.93 \mathrm{E}+01$ & $2.50 \mathrm{E}+01$ & $4.31 E+00$ \\
\hline PO4-3 & $3.20 \mathrm{E}+03$ & $2.80 \mathrm{E}+03$ & $3.93 \mathrm{E}+02$ & $1.85 E+03$ & $1.83 \mathrm{E}+03$ & $2.15 \mathrm{E}+01$ \\
\hline $\mathrm{Se}+6$ & $1.35 \mathrm{E}-04$ & & $1.35 \mathrm{E}-04$ & $3.31 \mathrm{E}+00$ & & $3.31 E+00$ \\
\hline $\mathrm{Si}+4$ & $1.59 \mathrm{E}+0 \mathrm{I}$ & $4.06 \mathrm{E}-01$ & $1.55 \mathrm{E}+01$ & $2.32 \mathrm{E}+02$ & $1.41 \mathrm{E}+01$ & $2.18 \mathrm{E}+02$ \\
\hline $\mathrm{SO} 4-2$ & $2.02 \mathrm{E}+03$ & $1.63 \mathrm{E}+03$ & $3.91 \mathrm{E}+02$ & $2.56 \mathrm{E}+01$ & 2. $19 \mathrm{E}+01$ & $3.65 E+00$ \\
\hline $\mathrm{Sr}+2$ & $3.80 \mathrm{E}-01$ & $3.62 \mathrm{E}-01$ & $1.86 \mathrm{E}-02$ & $3.66 \mathrm{E}+01$ & $3.60 \mathrm{E}+01$ & $6.00 \mathrm{E}-01$ \\
\hline TcO4- & $2.21 \mathrm{E}+00$ & $2.00 \mathrm{E}-01$ & $2.01 \mathrm{E}+00$ & $9.07 \mathrm{E}-01$ & $8.65 \mathrm{E}-01$ & $4.20 \mathrm{E}-02$ \\
\hline$T h+4$ & & & & $2.79 \mathrm{E}+00$ & & $2.79 \mathrm{E}+00$ \\
\hline $\mathrm{Ti}+4$ & & & & $1.55 \mathrm{E}-01$ & & $1.55 \mathrm{E}-01$ \\
\hline TOC & $1.06 \mathrm{E}+03$ & $2.26 \mathrm{E}+02$ & $8.31 \mathrm{E}+02$ & $8.73 \mathrm{E}+01$ & $1.51 \mathrm{E}+01$ & $7.22 \mathrm{E}+01$ \\
\hline $\mathrm{UO} 2+2$ & $1.06 \mathrm{E}+02$ & $9.67 \mathrm{E}+01$ & $9.27 \mathrm{E}+00$ & $1.54 \mathrm{E}+03$ & $1.50 \mathrm{E}+03$ & $3.36 \mathrm{E}+01$ \\
\hline $\mathrm{Zn}+2$ & $7.78 \mathrm{E}-04$ & & $7.78 \mathrm{E}-04$ & $5.06 \mathrm{E}-01$ & & $5.06 \mathrm{E}-01$ \\
\hline $\mathrm{ZrO} 2: 2 \mathrm{H} 2 \mathrm{O}$ & $7.53 \mathrm{E}+00$ & $6.68 \mathrm{E}+00$ & $8.56 \mathrm{E}-05$ & $1.20 \mathrm{E}+03$ & $6.61 \mathrm{E}+02$ & $5.34 \mathrm{E}+02$ \\
\hline
\end{tabular}


WHC-SD-WM-TI-784

Revision 0

Table 6-2. Radionuclide Inventory for Tank Waste Remediation Systems Reference Feed.

\begin{tabular}{|c|c|c|c|}
\hline Radionuclide & Soluble $(\mathrm{C})$ & Insoluble $(\mathrm{Ci})$ & Total $(\mathrm{Ci})$ \\
\hline${ }^{14} \mathrm{C}$ & $3.33 \mathrm{E}+03$ & $2.01 \mathrm{E}+03$ & $5.34 \mathrm{E}+03$ \\
\hline${ }^{90} \mathrm{Sr}^{*}$ & $1.88 \mathrm{E}+06$ & $5.17 \mathrm{E}+07$ & $5.36 \mathrm{E}+07$ \\
\hline${ }^{99} \mathrm{Tc}$ & $2.28 \mathrm{E}+04$ & $9.29 \mathrm{E}+03$ & $3.21 \mathrm{E}+04$ \\
\hline${ }^{137} \mathrm{Cs}{ }^{*}$ & $3.19 \mathrm{E}+07$ & $3.01 \mathrm{E}+06$ & $3.49 \mathrm{E}+06$ \\
\hline${ }^{237} \mathrm{~Np}$ & $6.95 \mathrm{E}+00$ & $6.25 \mathrm{E}+01$ & $6.94 \mathrm{E}+01$ \\
\hline${ }^{239} \mathrm{Pu}$ & $1.65 \mathrm{E}+03$ & $2.48 \mathrm{E}+04$ & $2.64 \mathrm{E}+04$ \\
\hline${ }^{240} \mathrm{Pu}$ & $4.30 \mathrm{E}+02$ & $6.28 \mathrm{E}+03$ & $6.71 \mathrm{E}+03$ \\
\hline${ }^{241} \mathrm{Pu}$ & $4.79 \mathrm{E}+03$ & $7.02 \mathrm{E}+04$ & $7.50 \mathrm{E}+04$ \\
\hline${ }^{241} \mathrm{Am}$ & $7.96 \mathrm{E}+03$ & $9.66 \mathrm{E}+04$ & $1.05 \mathrm{E}+05$ \\
\hline${ }^{244} \mathrm{Cm}$ & $1.20 \mathrm{E}+00$ & $1.19 \mathrm{E}+02$ & $1.20 \mathrm{E}+02$ \\
\hline Total & $3.38 \mathrm{E}+07$ & $5.49 \mathrm{E}+07$ & $8.87 \mathrm{E}+07$ \\
\hline
\end{tabular}

*Does not include daughter products. 


\section{WHC-SD-WM-TI-784. \\ Revision 0}

\subsection{REFERENCES}

Agnew, S. F., 1995 Hanford Defined Wastes: Chemical and Radionuclide Compositions, WHC-SD-WM-TI-632, Rev. 1, (LA-UR-94-2657), Los Alamos National Laboratory, Los Alamos, New Mexico.

Agnew, S. F. and J. G. Watkin, 1995, Estimation of Limiting Solubilities for Ionic Species in Hanford Waste Tank Supernates, LA-UR-94-3590, Los Alamos National Laboratory, Los Alamos, New Mexico.

Allen, G. K., 1976, Estimated Inventory of Chemicals Added to Underground Waste Tanks, 1944 through 1975, ARH-CD-610B, Atlantic Richfield Hanford Company, Richland, Washington.

Boldt, A. L., 1994, Personal Communication to M. J. Kưfer, Westinghouse Hanford Company, Richland, Washington

Boomer, K. D., S. K. Baker, A. L. Boldt, J. D. Galbraith, J. S. Garfield, C. E. Golberg, B. A. Higley, L. J. Johnson, M. J. Kupfer, R. M. Marusich, R. J. Parazin, A. N. Praga, G. W. Reddick, J. A. Reddick, E. J. Slaathaug, L. M. Swanson, T. L. Waldo, and C. E. Worcester, 1993, Tank Waste Technical Cptions Report, WHC-EP-0616, Rev. 0, Westinghouse Hanford Company, Richland, Washington.

Borsheim, G. L., 1994, Aluminum Usage at Hanford, Internal Memo 71310-94.017, Westinghouse Hanford Company, Richland, Washington.

Brevick, C. H., L. A. Gaddis, W. W. Pickett, 1994a, Historical Tank Content Estimate for the Northeast Quadrant of the Hanford 200 East Area, WHC-SD-WM-ER-349, Rev. 0 , ICF Kaiser Hanford Company, Richland, Washington.

Brevick, C. H., L. A. Gaddis, W. W. Pickett, 1994b, Historical Tank Content Estimate for the Southwest Quadrant of the Hanford 200 West Arta, WHC-SD-WM-ER-352, Rev. 0 , ICF Kaiser Hanford Company, Richland, Washing,ton.

Brevick, C. H., L. A. Gaddis, W. W. Pickett, 1994c, Supporting Document for North East Quadrant Historical Tank Content Estimate Report for AX-Tank Farm, WHC-SD-WM-ER-309, Rev, 0, ICF Kaiser Hanford Company, Richland, Washington.

Brevick, C. H., L. A. Gaddis, and E. D. Johnson, 1995, Historical Tank Content Estimate for the Northwest Quadrant of the Hanford 200 West Area, WHC-SD-WM-ER-351, Rev. 0, ICF Kaiser Hanford Company, Richland, Washington. 
WHC-SD-WM-TI-784.

Revision 0

Colton, N. G., 1995, Sludge Pretreatment Chemistry Evaluation: Enhanced Sludge Washing Separation Factors, PNL-10512, Pacific Northwest National Laboratory, Richland, Washington.

Ecology, 1994, Hanford Federal Facility Agreement and Consent Order, as amended, Washington State Department of Ecology, U.S. Environmental Protection Agency, and U.S. Department of Energy, Olympia, Washington.

ERDA, 1975, Final Environmental Statement, Hanford Waste Management Operations, ERDA-1538, U.S. Energy Research \& Development Administration, Washington, D.C.

Golberg, C. E. and Guberski, J. D., 1995, Single-Shell and Double-Shell Tank Waste Inventory Data Package for the Tank Waste Remediation System Environmental Impact Statement, WHC-SD-WM-EV-102, Westinghouse Hanford Company, Richland Washington.

Gray, W. J., M. E. Peterson, R. D. Scheele, J. M. Tingey, 1993a, Characterization of the Second Core Sample of Neutralized Current Acid Waste from Double-Shell Tank 101-AZ, Pacific Northwest National Laboratory, Richland, Washington.

Gray, W. J., M. E. Peterson, R. D. Scheele, J. M. Tingey, 1993b, Characterization of the First Core Sample of Neutralized Current Acid Waste from Double-Shell Tank I02-AZ, Pacific Northwest National Laboratory, Richland, Washington.

Hanlon, B. M., 1996, Tank Farm surveillance and Waste Status Summary Report for March 1995, WHC-EP-0182-96, Westinghouse Hanford Company, Richland, Washington.

Herting, D. L. 1994, Chemical Composition of Tank 101-SY Solids, Internal Memo to G. D. Johnson, (July 11, 1994), Westinghouse Hanford Company, Richland, Washington.

Jungfleisch, F. M., 1984, Track Radioactive Components Code, Rockwell Hanford Operations, Richland, Washington.

Kaiser, 1977, Hanford Defense High-Level Waste Management Studies, KE Report 77-09-RE, Kaiser Engineers, Richland, Washington.

Kupfer, M. J., 1996a, Interim Report: Best-Basis Inventories of Chemical and Radionuclides in Hanford Site Tank Waste, WHC-SD-WM-TI-740, Rev. C-Draft, Westinghouse Hanford Company, Richland, Washington.

Kupfer, M. J., 1996b, Work Plan for Defining a Standard Inventory Estimate for Wastes Stored in Hanford Site Underground Tanks, WHC-SD-WM-WP-311, Rev. 0, Westinghouse Hanford Company, Richland, Washington. 


\section{WHC-SD-WM-TI-784.}

Revision 0

LaFemina, J. P., D. L. Blanchard, P. J. Bruinsma, B. C. Bunker, I. E. Burgeson, Y. L. Chen, P. Colton, S. D. Conradson, A. R. Felmy, N. G. Gotts, G. L. Graff, N. J. Hess, J. C. Hutton, C. R. Hymas, M. M. Lamoureux, X. S. Li, J. Liu, G. J. Lumetta, J. R. Phillips, B. M. Rapko, D. R. Rector, J. R. Rustad, P. A. Smith, L. Song, S. M. Sterner, J. M. Tingey, S. M. Tingey, L. E. Thomas, L. Wang, Y. Wang, 1995, Tank Waste Treatment Science: Report for the Third Quarter FY 1995, TWRSPP-95-019, Pacific Northwest National Laboratory, Richland, Washington.

Lumetta, G. J., 1994, Pretreatment of Neutralized Cladding Removal Waste Sludge: Results of the Second Design Basis Experiment, PNL-9747, Pacific Northwest National Laboratory, Richland, Washington.

Lumetta, G. J. and J. L. Swanson, 1993, Pretreatment of Plutonium finishing Plant (PFP) Sludge: report for the Period October 1990 - March 1992, PNL-8601, Pacific Northwest National Laboratory, Richland, Washington.

Orme, R. M., 1995 TWRS Process Flowsheet, WHC-SD-WM-TI-613, Rev.1, Westinghouse Hanford Company, Richland, Washington.

ORNL, 1995, Integrated Data Base Report-1994: U.S. Spent Nuclear Fuel and Radioactive Waste Inventories, Projections, and Characteristics, DOE/RW-0006, Rev. 11, Oak Ridge National Laboratory, Oak Ridge, Tennessee.

Pajunen, A. L., 1994, Plutonium and Americium Inventory Estimates in Selected DoubleShell Tank Waste Types 7E360-94.007, Westinghous: Hanford Company, Richland, Washington.

Peterson, M., 1990, Revised Report on the Results of 102-AY Characterization, Internal Memo to A. J. DiLiberto, Battlle Pacific Northwest Laboratories, Richland, Washington.

RHO, 1985a, Hanford Defense Waste Disposal Alternatives: Engineering Support Data for the Hanford Defense Waste-Environmental Impact Statement, RHO-RE-ST-30P, Rockwell Hanford Company, Rockwell International, Richland, Washington.

RHO, 1980, Technical Aspects of Long-Term Management Alternatives for High-Level Defense Waste at the Hanford Site, RHO-LD-141, Rockwell Hanford Operations, Richland, Washington.

Schofield, J. S., 1991, Estimation of Neutralized Current Acid Waste and Neutralized Cladding Removal Waste Constituents, 85440-91-01\&, Westinghouse Hanford Company, Richland, Washington. 


\section{WHC-SD-WM-TI-784}

Revision 0

Schulz, W. W., 1978, Removal of Radionuclides from Hanford Defense Waste Solutions, RHO-SA-51, Rockwell Hanford Company, Rockwell International, Richland, Washington.

Sederburg, J. P., 1995, Waste Volume Reduction Factors for Potential 242-A Evaporator Feed, WHC-SD-WM-TI-690, Rev. 0, Westinghouse Hanford Company, Richland, Washington.

Strode, J. N.., 1995, Operational Waste Volume Projection, WHC-SD-WM-ER-029, Rev. 21, Westinghouse Hanford Company, Richland, Washington. 
WHC-SD-WM-TI-784

Revision 0

\section{APPENDIX A}

\section{CHEMICAL AND RADIONUCLIDE INVENTORY ESTIMATES FOR SINGLE- AND DOUBLE-SHELL TANKS}


WHC-SD-WM-TI-784

Revision 0

This page intentionally left blank.

A-2 
Table A-1. Chemical Inventory Estimates for Single-Shell Tanks

\begin{tabular}{|c|c|c|c|c|c|c|c|c|}
\hline $\begin{array}{c}\text { Soluble } \\
\text { (kg) }\end{array}$ & A-101 & A-102 & A-103 & A-104 & A-105 & A-106 & $A X-101$ & $A X-102$ \\
\hline $\mathrm{Ag}+$ & $N / A$ & N/A & N/A & N/A & IN/A & $\mathrm{N} / \mathrm{A}$ & N/A & $N / A$ \\
\hline $\mathrm{Al}(\mathrm{OH}) 4$ & $8.97 E+04$ & $3.01 E+03$ & $2.81 E+04$ & & & $2.97 E+03$ & $6.47 E+04$ & $2.67 E+03$ \\
\hline $\mathrm{As}+5$ & N/A & N/A & N/A & N/A & IV/A & $\mathrm{N} / \mathrm{A}$ & N/A & N/A \\
\hline $\mathrm{B}+3$ & N/A & N/A & $N / A$ & $N / A$ & N $/ A$ & $\mathrm{~N} / \mathrm{A}$ & N/A & N/A \\
\hline $\mathrm{Ba}+2$ & $N / A$ & N/A & N/A & N/A & IN/A & $\mathrm{N} / \mathrm{A}$ & N/A & $N / A$ \\
\hline $\mathrm{Be}+2$ & N/A & $\mathrm{N} / \mathrm{A}$ & $N / A$ & N/A & IN/A & N/A & N/A & N/A \\
\hline $\mathrm{Bi}+3$ & $3.01 E+02$ & $1.08 E+01$ & $9.45 E+01$ & & & $2.36 \mathrm{E}+01$ & $2.21 \mathrm{E}+02$ & $8.19 E+00$ \\
\hline $\mathrm{Ca}+2$ & $1.27 E+02$ & $4.52 E+00$ & $6.09 E+01$ & $2.69 \mathrm{E}-01$ & $1.84 E-01$ & $1.05 E+01$ & $1.06 E+02$ & $5.13 E+00$ \\
\hline $\mathrm{Cd}+2$ & N/A & N/A & N/A & N/A & IN/A & $\mathrm{N} / \mathrm{A}$ & N/A & N/A \\
\hline $\mathrm{Ce}+3$ & & $1.52 E+01$ & 5.61E-01 & & $1.06 \mathrm{E}-05$ & & & \\
\hline Cl. & $1.76 \mathrm{E}+04$ & $6.56 E+02$ & $6.42 E+03$ & $7.03 E+01$ & $5.90 \mathrm{E}+00$ & $1.69 E+03$ & $1.35 E+04$ & $5.51 E+02$ \\
\hline $\mathrm{Co}+3$ & N/A & $\mathbf{N} / \mathbf{A}$ & N/A & N/A & IN/A & N/A & N/A & N/A \\
\hline $\mathrm{CO}-2$ & $5.19 E+04$ & $1.97 \varepsilon+03$ & $1.79 E+04$ & $4.16 E+02$ & $2.83 E+02$ & $5.51 E+03$ & $3.92 \mathrm{E}+04$ & $2.05 E+03$ \\
\hline $\mathrm{Cr}(\mathrm{OH}) 4-$ & $4.87 E+03$ & $1.75 E+02$ & $1.48 E+03$ & $5.78 E+00$ & $3.58 \mathrm{E}-01$ & $3.87 E+02$ & $3.55 \mathrm{E}+03$ & $1.30 E+02$ \\
\hline $\mathrm{Cs}+$ & & $5.39 E+00$ & $3.52 \mathrm{E}-01$ & & $6.44 \mathrm{E}-01$ & & & \\
\hline $\mathrm{Cu}+2$ & $N / A$ & N/A & N/A & N/A & IN/A & $\mathrm{N} / \mathrm{A}$ & $\mathrm{N} / \mathrm{A}$ & $N / A$ \\
\hline $\mathrm{F}$ - & $1.51 E+04$ & $5.41 E+02$ & $4.85 \mathrm{E}+03$ & & & $1.18 E+03$ & $1.11 E+04$ & $4.18 \mathrm{E}+02$ \\
\hline $\mathrm{Fe}+3$ & $3.54 E+02$ & $1.35 \mathrm{E}+01$ & $1.67 \mathrm{E}+02$ & $1.55 \mathrm{E}+01$ & $3.88 \mathrm{E}+01$ & $4.95 E+01$ & $3.09 E+02$ & $2.94 E+01$ \\
\hline $\mathrm{H} 2 \mathrm{O}$ & $1.31 E+07$ & $4.82 \mathrm{E}+05$ & $4.93 E+06$ & $1.55 E+05$ & $2.76 \mathrm{E}+05$ & $1.23 E+06$ & $1.02 E+07$ & $4.13 E+05$ \\
\hline $\mathrm{Hg}+2$ & N/A & N/A & N/A & N/A & W/A & N/A & N/A & N/A \\
\hline $\mathrm{K}+$ & $2.87 \mathrm{E}+03$ & $1.03 E+02$ & $9.37 \mathrm{E}+02$ & & & $2.26 \mathrm{E}+02^{\circ}$ & $2.13 E+03$ & $8.04 \mathrm{E}+01$ \\
\hline \multicolumn{9}{|l|}{$\mathrm{La}+3$} \\
\hline $\mathrm{Mg}+2$ & $\mathrm{~N} / \mathrm{A}$ & $N / A$ & N/A & $N / A$ & N $/ \mathbf{A}$ & N/A & N/A & $N / A$ \\
\hline $\mathrm{Mn}+4$ & $4.74 \mathrm{E}+01$ & $1.70 E+00$ & $1.69 E+01$ & & & $3.74 E+00$ & $3.60 \mathrm{E}+01$ & $1.42 E+00$ \\
\hline $\mathrm{Mo}+6$ & $N / A$ & N/A & $N / A$ & N/A & N $/ A$ & N/A & N/A & $N / A$ \\
\hline $\mathrm{Na}+$ & $1.68 \mathrm{E}+06$ & $6.17 E+04$ & $6.38 \mathrm{E}+05$ & $1.77 \mathrm{E}+04$ & 3.1 $\mathrm{NE}+\mathrm{O} 4$ & $1.57 \mathrm{E}+05$ & $1.31 E+06$ & $5.34 E+04$ \\
\hline $\mathrm{Ni}+3$ & $7.85 E+01$ & 2.77E + 00 & $3.76 \mathrm{E}+01$ & $4.21 E-01$ & $2.85 \mathrm{E}-01$ & $6.54 E+O O$ & $6.52 E+01$ & $3.03 E+00$ \\
\hline $\mathrm{NO} 2-$ & $3.19 \mathrm{E}+05$ & $1.15 E+04$ & $1.01 E+05$ & $1.50 \mathrm{E}+\infty 0$ & $3.09 \mathrm{E}+00$ & $2.51 \mathrm{E}+04$ & $2.34 E+05$ & $8.76 \mathrm{E}+03$ \\
\hline NO3. & $2.35 E+O B$ & $8.40 E+04$ & $1.01 \mathrm{E}+06$ & $8.71 E+03$ & $5.87 \mathrm{~F}+02$ & $1.93 \mathrm{E}+05$ & $1.88 E+06$ & $8.30 E+04$ \\
\hline $\mathrm{OH}-$ & $4.21 E+05$ & $1.64 E+04$ & $1.37 E+05$ & $1.14 \mathrm{E}+04$ & $2.21 E+04$ & $5.09 E+04$ & $3.21 E+05$ & $1.14 E+04$ \\
\hline $\mathrm{Pb}+4$ & $2.73 E+02$ & $9.81 E+00$ & $8.63 E+01$ & & & $2.14 \mathrm{E}+01$ & $2.01 E+02$ & $7.48 \mathrm{E}+00$ \\
\hline PO4-3 & $4.77 \mathrm{E}+04$ & $1.71 E+03$ & $1.50 E+04$ & 8.31E + 01 & & $3.81 \mathrm{E}+\mathrm{O} 3$ & $3.50 \mathrm{E}+04$ & $1.32 E+03$ \\
\hline$R b+$ & N/A & N/A & $\mathrm{N} / \mathrm{A}$ & N/A & $\mathbf{N} / \mathbf{A}$ & N/A & N/A & $\mathrm{N} / \mathrm{A}$ \\
\hline$R e+7$ & $\mathrm{~N} / \mathrm{A}$ & N/A & N/A & N/A & $N / A$ & N/A & $N / A$ & N/A \\
\hline $\mathrm{Rh}+3$ & N/A & $N / A$ & N/A & N/A & N/A & N/A & N/A & N/A \\
\hline Ru + 3 & $\mathrm{~N} / \mathrm{A}$ & N/A & N/A & $N / A$ & $\mathrm{~N} / \mathrm{A}$ & $N / A$ & N/A & N/A \\
\hline $\mathrm{Se}_{\theta}+6$ & $\mathrm{~N} / \mathrm{A}$ & N/A & N/A & N/A & N/A & $N / A$ & $N / A$ & $N / A$ \\
\hline \multicolumn{9}{|l|}{$\mathrm{Si}+4$} \\
\hline SO4-2 & $5.06 \mathrm{E}+04$ & $1.82 \mathrm{E}+03$ & $2.44 E+04$ & $1.17 E+02$ & 8.1 $E \mathrm{E}+03$ & $4.44 \mathrm{E}+03$ & $4.63 E+04$ & $1.95 \mathrm{E}+03$ \\
\hline $\mathrm{sr}+2$ & $1.64 \mathrm{E}-01$ & $3.55 E-01$ & $9.62 \mathrm{E}-04$ & $1.93 \mathrm{E}-01$ & $1.57 \mathrm{E}-02$ & & $8.27 E-03$ & $9.46 \mathrm{E}-03$ \\
\hline$T_{e}+6$ & N/A & $N / A$ & N/A & N/A & $\mathrm{N} / \mathrm{A}$ & $N / A$ & N/A & N/A \\
\hline$T h+4$ & $N / A$ & N/A & $N / A$ & N/A & $\mathrm{N} / \mathrm{A}$ & N/A & N/A & N/A \\
\hline$T I+4$ & $N / A$ & $\mathrm{~N} / \mathrm{A}$ & N/A & $\mathrm{N} / \mathrm{A}$ & $\mathrm{N} / \mathrm{A}$ & $N / A$ & N/A & N/A \\
\hline TOC & $3.68 E+04$ & $2.39 E+03$ & $1.16 E+04$ & & & $1.25 E+04$ & $2.82 \mathrm{E}+04$ & $1.01 E+03$ \\
\hline $\mathrm{UO} 2+2$ & $5.16 \mathrm{E}+02$ & $1.62 \mathrm{E}+01$ & $2.31 E+02$ & $1.88 \mathrm{E}+01$ & $6.07 \mathrm{E}+02$ & $3.66 \mathrm{E}+01$ & $7.07 E+02$ & $1.80 E+01$ \\
\hline$Z n+2$ & N/A & N/A & N/A & N/A & $\mathrm{N} / \mathrm{A}$ & $N / A$ & N/A & N/A \\
\hline \multicolumn{9}{|l|}{$\mathrm{ZrO} 2 ; 2 \mathrm{H} 2 \mathrm{O}$} \\
\hline Total & $1.82 \mathrm{E}+07$ & $6.68 \mathrm{E}+05$ & $6.92 \mathrm{E}+06$ & $9.94 E+05$ & $3.3 \mathrm{E}+05$ & $1.69 \mathrm{E}+06$ & $1.42 \mathrm{E}+07$ & $5.80 E+05$ \\
\hline
\end{tabular}


Table A-1. Chemical Inventory Estimates for Single-Shell Tanks

\begin{tabular}{|c|c|c|c|c|c|c|c|c|}
\hline $\begin{array}{c}\text { Soluble } \\
(\mathrm{kg})\end{array}$ & $A X-103$ & AX-104 & B-101 & 8-102 & B. 103 & B-104 & B-105 & B-106 \\
\hline $\mathrm{Ag}+$ & $N / A$ & N/A & N/A & N/A & IN/A & $N / A$ & N/A & N/A \\
\hline Al(OH)4- & $6.06 E+03$ & & $9.16 E+02$ & $1.85 E+02$ & & $4.17 \mathrm{E}+\mathrm{O} 3$ & $2.29 \mathrm{E}+02$ & \\
\hline As + 5 & N/A & $N / A$ & $\mathrm{~N} / \mathrm{A}$ & N/A & IN/A & N/A & N/A & N/A \\
\hline$B+3$ & N/A & N/A & N/A & N/A & IN/A & $N / A$ & N/A & N/A \\
\hline $\mathrm{Ba}+2$ & N/A & N/A & $\mathrm{N} / \mathrm{A}$ & N/A & IJ/A & $N / A$ & $N / A$ & N/A \\
\hline $\mathrm{Be}+2$ & N/A & N/A & N/A & $N / A$ & $\mathrm{IN} / \mathrm{A}$ & N/A & N/A & N/A \\
\hline $8 i+3$ & $2.55 \mathrm{E}+01$ & & $1.42 \mathrm{E}+02$ & $4.54 E+01$ & $1.06 \mathrm{iE}+02$ & $1.89 E+02$ & $5.37 \mathrm{E}+02$ & $2.19 E+02$ \\
\hline $\mathrm{Ca}+2$ & $1.65 E+01$ & $6.78 \mathrm{E}-02$ & $3.16 \mathrm{E}+01$ & $9.87 E+00$ & 2.30E + 01 & $2.61 E+01$ & $1.14 E+02$ & $4.76 \mathrm{E}+01$ \\
\hline $\mathrm{Cd}+2$ & N/A & N/A & N/A & $\mathrm{N} / \mathrm{A}$ & $\mathrm{N} / \mathrm{A}$ & N/A & N/A & $\mathrm{N} / \mathrm{A}$ \\
\hline $\mathrm{Ce}+3$ & $1.77 E+00$ & $1.85 \mathrm{E}-02$ & & $2.65 E-01$ & $9.10 \mathrm{DE}-01$ & $2.02 E+01$ & $1.50 \mathrm{E}+00$ & $1.75 \mathrm{E}-01$ \\
\hline Cl- & $1.73 E+03$ & $2.21 \mathrm{E}+00$ & $9.77 E+02$ & $2.37 E+02$ & $5.4 \varepsilon E+02$ & $1.16 \mathrm{E}+03$ & $2.80 \mathrm{E}+03$ & $1.13 E+03$ \\
\hline $\mathrm{Co}+3$ & N/A & N/A & N/A & $\mathrm{N} / \mathrm{A}$ & $\mathrm{N} / \mathrm{A}$ & N/A & N/A & $N / A$ \\
\hline $\mathrm{CO}-2$ & $5.02 E+03$ & $1.05 \mathrm{E}+02$ & $4.68 \mathrm{E}+03$ & $1.08 E+03$ & $2.05 \mathrm{E}+03$ & $3.55 \mathrm{E}+03$ & $9.17 E+03$ & $3.73 E+03$ \\
\hline $\mathrm{Cr}(\mathrm{OH}) 4$ & $4.00 \mathrm{E}+02$ & 1.32E-01 & $1.51 \mathrm{E}+02$ & $4.86 \mathrm{E}+01$ & 1.1:E+O2 & $1.65 E+02$ & $5.64 E+02$ & $2.32 \mathrm{E}+02$ \\
\hline Cs + & 3.09E-01 & $2.09 E+00$ & & $4.42 \mathrm{E}-02$ & $1.79 \mathrm{E}-01$ & $8.35 \mathrm{E}-02$ & $6.95 \mathrm{E}-01$ & $3.15 E-02$ \\
\hline $\mathrm{Cu}+2$ & $\mathrm{~N} / \mathrm{A}$ & N/A & N/A & N/A & $\mathrm{N} / \mathrm{A}$ & N/A & N/A & N/A \\
\hline $\mathrm{F}-$ & $1.31 \mathrm{E}+03$ & & $6.59 E+03$ & $2.11 E+03$ & $4.9 \mathrm{E}+03$ & $1.54 E+04$ & $2.58 \mathrm{E}+04$ & $1.02 \mathrm{E}+04$ \\
\hline $\mathrm{Fe}+3$ & $7.34 E+01$ & $1.43 \mathrm{E}+01$ & $1.29 E+02$ & $2.80 E+01$ & $6.31 \mathrm{E}+01$ & $1.46 \mathrm{E}+02$ & $3.22 \mathrm{E}+\mathrm{O} 2$ & $1.30 \mathrm{E}+02$ \\
\hline $\mathrm{H} 2 \mathrm{O}$ & $1.48 E+06$ & $1.02 E+05$ & $1.19 E+06$ & $3.49 E+05$ & $7.8 j E+05$ & $2.38 \mathrm{E}+06$ & $3.99 \mathrm{E}+0.6$ & $1.59 E+06$ \\
\hline $\mathrm{Hg}+2$ & N/A & N/A & N/A & N/A & $\mathrm{N} / \mathrm{A}$ & N/A & N/A & $N / A$ \\
\hline $\mathrm{K}+$ & $2.53 E+02$ & & & & & & & \\
\hline \multicolumn{9}{|l|}{$\mathrm{La}+3$} \\
\hline $\mathrm{Mg}+2$ & N/A & N/A & N/A & N/A & N/A & N/A & N/A & N/A \\
\hline$M n+4$ & $4.56 \mathrm{E}+00$ & & & & & & & \\
\hline Mo +6 & $\mathrm{N} / \mathrm{A}$ & $N / A$ & N/A & N/A & N/A & N/A & N/A & N/A \\
\hline $\mathrm{Na}+$ & $1.89 \mathrm{E}+05$ & $1.15 E+04$ & $1.45 E+05$ & $4.26 E+04$ & $9.61 E+04$ & $2.80 E+05$ & $4.87 E+05$ & $1.94 \mathrm{E}+05$ \\
\hline $\mathrm{Ni}+3$ & $1.03 E+01$ & $1.05 \mathrm{E}-01$ & $2.16 E+01$ & $6.11 E+00$ & $1.4 E E+01$ & $1.69 E+01$ & $7.07 E+01$ & $2.93 \mathrm{E}+01$ \\
\hline $\mathrm{NO} 2-$ & $2.73 E+04$ & $1.14 E+00$ & $2.96 \mathrm{E}+03$ & $9.72 \mathrm{E}+0.2$ & $2.16 E+03$ & $5.21 E+03$ & $1.12 \mathrm{E}+04$ & $-4.48 \mathrm{E}+03$ \\
\hline NO3. & $2.69 E+05$ & $2.17 \mathrm{E}+02$ & $1.38 E+05$ & $3.87 E+04$ & $8.96 E+04$ & $1.72 \mathrm{E}+05$ & $4.53 \mathrm{E}+05$ & $1.85 E+05$ \\
\hline $\mathrm{OH}-$ & $4.98 \mathrm{E}+04$ & $8.15 E+03$ & $3.66 \mathrm{E}+04$ & $1.02 E+04$ & $2.24 \cdot E+04$ & $1.02 E+05$ & $1.13 E+05$ & $4.35 E+04$ \\
\hline $\mathrm{Pb}+4$ & $2.33 \mathrm{E}+01$ & & $3.52 E-04$ & & & & & \\
\hline PO4-3 & $4.05 E+03$ & & $4.70 \mathrm{E}+04$ & $1.52 E+04$ & $3.51 \mathrm{E}+04$ & $8.74 \mathrm{E}+04$ & $1.80 E+05$ & $7.22 E+04$ \\
\hline $\mathrm{Rb}+$ & N/A & N/A & N/A & N/A & N/A & $\mathrm{N} / \mathrm{A}$ & N/A & $\mathrm{N} / \mathrm{A}$ \\
\hline$R e+7$ & N/A & $\mathrm{N} / \mathrm{A}$ & N/A & $N / A$ & N/A & N/A & N/A & N/A \\
\hline$R h+3$ & $\mathrm{~N} / \mathrm{A}$ & N/A & N/A & N/A & N/A & N/A & N/A & N/A \\
\hline$R u+3$ & $\mathrm{~N} / \mathrm{A}$ & N/A & N/A & N/A & N/A & N/A & N/A & N/A \\
\hline $\mathrm{Se}+6$ & $\mathrm{~N} / \mathrm{A}$ & N/A & $\mathrm{N} / \mathrm{A}$ & $\mathrm{N} / \mathrm{A}$ & $\mathrm{M} / \mathrm{A}$ & N/A & N/A & N/A \\
\hline $\mathrm{Si}+4$ & & & & & $3.7 \mathrm{C} E+01$ & & & 7.64E +01 \\
\hline so4-2 & $1.26 E+04$ & $3.02 E+03$ & $7.26 E+03$ & $2.45 E+03$ & $5.22 E+03$ & $6.52 E+03$ & $2.43 E+04$ & $1.00 E+04$ \\
\hline$S_{r}+2$ & $1.26 \mathrm{E}-\mathrm{O} 2$ & $4.65 \mathrm{E}-01$ & & $1.62 \mathrm{E}-05$ & 4.8:5E-04 & $4.00 \mathrm{E}-04$ & $6.00 \mathrm{E}-05$ & \\
\hline$T_{6}+6$ & N/A & N/A & $N / A$ & $N / A$ & N/A & N/A & N/A & N/A \\
\hline Th+ 4 & N/A & N/A & $N / A$ & $\mathrm{~N} / \mathrm{A}$ & $\mathrm{M} / \mathrm{A}$ & N/A & N/A & N/A \\
\hline $\mathrm{TI}+4$ & $N / A$ & N/A & $N / A$ & $\mathrm{~N} / \mathrm{A}$ & $N / A$ & $\mathrm{~N} / \mathrm{A}$ & $\mathrm{N} / \mathrm{A}$ & $N / A$ \\
\hline TOC & $3.24 \mathrm{E}+03$ & & $6.54 \mathrm{E}+02$ & & & & & \\
\hline $\mathrm{NO} 2+2$ & $5.10 \mathrm{E}+02$ & $2.24 E+O 2$ & $2.34 E+03$ & $1.81 \mathrm{E}+02$ & $1.72 \mathrm{E}+02$ & $9.00 E+00$ & $2.70 E+01$ & $1.11 \mathrm{E}+01$ \\
\hline$Z n+2$ & $\mathrm{~N} / \mathrm{A}$ & N/A & N/A & N/A & $\mathrm{N} / \mathrm{A}$ & $N / A$ & $N / A$ & $\mathrm{~N} / \mathrm{A}$ \\
\hline $\mathrm{ZrO} 2: 2 \mathrm{H} 2 \mathrm{O}$ & & & & & & $3.89 \mathrm{E}+01$ & $5.15 E+00$ & \\
\hline Total & $2.05 E+06$ & $1.25 E+05$ & $1.58 \mathrm{E}+06$ & $4.63 E+05$ & $1.05 E+06$ & $3.06 \mathrm{E}+06$ & $5.30 \mathrm{E}+06$ & $2.11 E+06$ \\
\hline
\end{tabular}


WHC-SD-WM-TI-784

Revision 0

Table A-1. Chemical Inventory Estimates for Single-Shell Tanks

\begin{tabular}{|c|c|c|c|c|c|c|c|c|}
\hline $\begin{array}{c}\text { Soluble } \\
\text { (kg) }\end{array}$ & B-107 & B- 108 & B-109 & B-110 & B-111 & B-112 & B-201 & $\mathrm{B}-2 \mathrm{O} 2$ \\
\hline $\mathrm{Ag}+$ & N/A & N/A & $\mathrm{N} / \mathrm{A}$ & N/A & $N / A$ & $\mathrm{~N} / \mathrm{A}$ & N/A & N/A \\
\hline Al(OH)4 & $6.91 E+03$ & $1.40 \mathrm{E}+03$ & $2.56 E+03$ & & & $6.35 \mathrm{E}+02$ & & \\
\hline$A s+5$ & N/A & N/A & $N / A$ & N/A & IV/A & $\mathrm{N} / \mathrm{A}$ & $N / A$ & $N / A$ \\
\hline$B+3$ & N/A & $N / A$ & N/A & N/A & IV/A & N/A & N/A & N/A \\
\hline $\mathrm{Ba}+2$ & N/A & $\mathrm{N} / \mathrm{A}$ & N/A & N/A & IV/A & N/A & N/A & $N / A$ \\
\hline $\mathrm{Be}+2$ & N/A & $\mathbf{N} / \mathbf{A}$ & N/A & N/A & IN/A & N/A & N/A & $N / A$ \\
\hline$B i+3$ & $4.74 E+01$ & $1.23 E+02$ & $1.59 E+02$ & $9.54 E+0 t$ & $8.77 E+01$ & $9.68 \mathrm{E}+00$ & $1.06 \mathrm{E}+01$ & $1.03 E+07$ \\
\hline $\mathrm{Ca}+2$ & $5.81 \mathrm{E}-01$ & $2.47 E+01$ & $3.46 E+01$ & $1.49 E+00$ & $1.5 ! 5 E+00$ & $6.37 E+\infty 0$ & $2.73 \mathrm{E}-01$ & 2.64E-01 \\
\hline $\mathrm{Cd}+2$ & $N / A$ & N/A & N/A & N/A & IV/A & N/A & $\mathrm{N} / \mathrm{A}$ & $\mathrm{N} / \mathrm{A}$ \\
\hline $\mathrm{C}_{\theta}+3$ & $9.21 E+00$ & $1.85 E+00$ & $1.06 \mathrm{E}+00$ & & $3.62 \mathrm{E}-02$ & $1.25 E+01$ & & \\
\hline Cl: & $1.88 E+03$ & $6.47 E+02$ & $8.41 E+02$ & $8.61 E+02$ & $7.915 \mathrm{E}+02$ & $2.73 E+02$ & $1.12 \mathrm{E}+02$ & $1.09 \mathrm{E}+02$ \\
\hline $\mathrm{Co}+3$ & N/A & N/A & $\mathrm{N} / \mathrm{A}$ & $\mathrm{N} / \mathrm{A}$ & IN/A & $\mathrm{N} / \mathrm{A}$ & $\mathrm{N} / \mathrm{A}$ & $\mathrm{N} / \mathrm{A}$ \\
\hline $\cos -2$ & $8.97 E+02$ & $2.12 \mathrm{E}+03$ & $2.95 E+03$ & $2.30 E+03$ & $2.39 \mathrm{E}+03$ & $7.75 E+02$ & $4.21 E+02$ & $4.07 E+02$ \\
\hline $\mathrm{Cr}(\mathrm{OH}) 4$ & $3.21 E+01$ & $1.27 \mathrm{E}+02$ & $1.71 \mathrm{E}+02$ & $4.73 E+01$ & 4.3: $E+01$ & $2.03 E+02$ & $6.18 \mathrm{E}+00$ & $5.97 E+00$ \\
\hline Cs + & 8.14E-01 & $5.70 E-01$ & 2.3OE-01 & $1.14 \mathrm{E}+00$ & 1.0:2E + 01 & $4.18 E+00$ & & \\
\hline $\mathrm{Cu}+2$ & N/A & N/A & N/A & N/A & IN/A & N/A & N/A & $\mathrm{N} / \mathrm{A}$ \\
\hline F- & $6.84 E+03$ & $6.68 \varepsilon+03$ & $7.38 \mathrm{E}+03$ & $1.23 \mathrm{E}+04$ & $1.23 E+04$ & $9.29 E+02$ & $8.71 E+03$ & $8.42 E+03$ \\
\hline $\mathrm{Fe}+3$ & $4.20 \mathrm{E}+01$ & $7.58 \mathrm{E}+01$ & $1.03 E+02$ & $1.18 \mathrm{E}+02$ & $1.3+\mathrm{HE}+02$ & $2.39 \mathrm{E}+01$ & $1.40 \mathrm{E}+01$ & $1.36 \mathrm{E}+01$ \\
\hline $\mathrm{H} 2 \mathrm{O}$ & $1.19 E+06$ & $1.06 \mathrm{E}+06$ & $1.16 E+06$ & $1.77 E+0 B$ & $1.7 \mathrm{SE}+06$ & $3.44 E+05$ & 1.77E + 05 & $1.71 E+05$ \\
\hline $\mathrm{Hg}+2$ & N/A & N/A & N/A & N/A & IN/A & N/A & $\mathrm{N} / \mathrm{A}$ & N/A \\
\hline $\mathrm{K}+$ & & & & & & $3.42 \mathrm{E}+01$ & $2.39 \mathrm{E}+02$ & $2.31 \varepsilon+02$ \\
\hline $\mathrm{La}+3$ & & & & & & & $1.63 \mathrm{E}+00$ & $1.58 \mathrm{E}+00$ \\
\hline $\mathrm{Mg}+2$ & N/A & $N / A$ & $N / A$ & N/A & IJ/A & N/A & $N / A$ & N/A \\
\hline $\mathrm{Mn}+4$ & & & & & & & $3.19 E-02$ & $3.08 \mathrm{E}-02$ \\
\hline $\mathrm{Mo}+6$ & N/A & N/A & N/A & N/A & IN/A & $\mathrm{N} / \mathrm{A}$ & N/A & N/A \\
\hline $\mathrm{Na}+$ & $1.37 \mathrm{E}+05$ & $1.28 E+05$ & $1.43 E+05$ & $2.08 E+05$ & $2.0 \$ E+05$ & $4.46 \mathrm{E}+04$ & $2.15 E+04$ & $2.08 E+04$ \\
\hline $\mathrm{Ni}+3$ & 7.57E-01 & $1.53 \mathrm{E}+01$ & $2.15 E+01$ & $2.17 E+00$ & 2.1'E + 00 & $4.05 E+\infty 0$ & $4.21 E-01$ & $4.07 \mathrm{E}-01$ \\
\hline NO2- & $3.85 \mathrm{E}+03$ & $3.12 \mathrm{E}+03$ & $3.82 \mathrm{E}+03$ & $3.25 \mathrm{E}-01$ & $3.54 E+00$ & $3.84 E+03$ & & \\
\hline NO3- & $3.56 E+04$ & $1.03 E+05$ & $1.38 \mathrm{E}+05$ & $1.27 E+05$ & $1.11 \mathrm{E}+05$ & $7.67 E+04$ & $2.12 E+04$ & $2.05 E+04$ \\
\hline $\mathrm{OH}$. & $6.47 E+04$ & $3.56 \mathrm{E}+04$ & $3.10 \mathrm{E}+04$ & $8.56 E+04$ & $9.15 E+04$ & $7.47 E+03$ & $1.50 \mathrm{E}+03$ & $1.45 E+03$ \\
\hline $\mathrm{Pb}+4$ & & & & & $7.06 \mathrm{E}-05$ & $4.32 E+00$ & & \\
\hline $\mathrm{PO} 4-3$ & $4.01 E+04$ & $4.57 E+04$ & $5.24 \mathrm{E}+04$ & $4.85 E+04$ & $4.4 E E+04$ & $3.62 \mathrm{E}+03$ & $7.72 \mathrm{E}+02$ & $7.46 \mathrm{E}+02$ \\
\hline $\mathbf{R b}+$ & N/A & N/A & $\mathrm{N} / \mathrm{A}$ & N/A & $\mathrm{N} / \mathrm{A}$ & $\mathrm{N} / \mathrm{A}$ & $\mathrm{N} / \mathrm{A}$ & N/A \\
\hline$R e+7$ & $N / A$ & N/A & $\mathrm{N} / \mathrm{A}$ & N/A & NI/A & $\mathrm{N} / \mathrm{A}$ & N/A & N/A \\
\hline$R h+3$ & N/A & $N / A$ & N/A & $\mathrm{N} / \mathrm{A}$ & N/A & $\mathrm{N} / \mathrm{A}$ & $N / A$ & N/A \\
\hline$R u+3$ & N/A & $N / A$ & N/A & N/A & $\mathrm{N} / \mathrm{A}$ & $\mathrm{N} / \mathrm{A}$ & N/A & N/A \\
\hline Se+6 & N/A & N/A & N/A & N/A & N/A & N/A & N/A & N/A \\
\hline \multicolumn{9}{|l|}{$\mathrm{Si}+4$} \\
\hline SO4-2 & $9.55 E+02$ & $5.39 \mathrm{E}+03$ & $7.28 \mathrm{E}+03$ & $2.13 E+03$ & $6.8 \mathrm{C} E+03$ & $1.32 \mathrm{E}+03$ & $1.29 \mathrm{E}+01$ & $1.25 \mathrm{E}+01$ \\
\hline$s r+2$ & $1.19 \mathrm{E}-03$ & $2.12 \mathrm{E}-03$ & $2.76 E-04$ & $4.74 E-03$ & $1.0 .3 \mathrm{E}-02$ & $6.67 \mathrm{E}-03$ & $3.11 E+01$ & $3.00 E+01$ \\
\hline$T e+6$ & N/A & $\mathrm{N} / \mathrm{A}$ & N/A & N/A & $M / A$ & N/A & N/A & N/A \\
\hline Th +4 & N/A & $N / A$ & N/A & N/A & $N / A$ & $\mathrm{~N} / \mathrm{A}$ & $N / A$ & $N / A$ \\
\hline $\mathrm{TI}+4$ & N/A & N/A & N/A & N/A & Al/A & N/A & $\mathrm{N} / \mathrm{A}$ & $N / A$ \\
\hline TOC & & & & & $2.45 E+00$ & $1.64 E+02$ & $7.64 \mathrm{E}+02$ & $7.39 \mathrm{E}+02$ \\
\hline $\mathrm{UO} 2+2$ & $3.85 E+00$ & $6.53 E+00$ & $1.55 E+02$ & $6.46 E+01$ & $4.18 E+02$ & $2.26 \mathrm{E}+01$ & & \\
\hline$Z n+2$ & $\mathrm{~N} / \mathrm{A}$ & N/A & $N / A$ & $N / A$ & N/A & $\mathrm{N} / \mathrm{A}$ & $N / A$ & $N / A$ \\
\hline $\mathrm{ZrO} 2: 2 \mathrm{H} 2 \mathrm{O}$ & $5.27 \mathrm{E}+01$ & $1.09 \mathrm{E}+01$ & & & & & & \\
\hline Total & $1.49 E+06$ & $1.40 E+06$ & $1.55 \mathrm{E}+06$ & $2.26 E+06$ & $2.28 E+06$ & $4.85 E+05$ & $2.32 E+05$ & $2.25 \mathrm{E}+05$ \\
\hline
\end{tabular}


WHC-SD-WM-TI-784

Revision 0

Table A-1. Chemical Inventory Estimates for Single-Shell Tanks

\begin{tabular}{|c|c|c|c|c|c|c|c|c|}
\hline $\begin{array}{c}\text { Soluble } \\
(\mathrm{kg})\end{array}$ & B-203 & B-204 & $\mathrm{BX}-101$ & $\mathrm{BX}-102$ & B):-103 & BX-104 & $B \times-105$ & BX-106 \\
\hline $\mathrm{Ag}+$ & $\mathrm{N} / \mathrm{A}$ & N/A & N/A & N/A & IN/A & N/A & N/A & N/A \\
\hline Al(OH)4- & & & $3.37 E+02$ & $9.92 \mathrm{E}+02$ & $1.5: \mathrm{EE}+03$ & & & \\
\hline As + 5 & N/A & N/A & N/A & N/A & IN/A & N/A & N/A & N/A \\
\hline$B+3$ & N/A & N/A & N/A & $N / A$ & IV/A & N/A & N/A & N/A \\
\hline $\mathrm{Ba}+2$ & N/A & N/A & N/A & N/A & IN/A & N/A & N/A & $N / A$ \\
\hline $\mathrm{Be}+2$ & N/A & $\mathrm{N} / \mathrm{A}$ & $N / A$ & N/A & $\mathrm{IN} / \mathrm{A}$ & N/A & N/A & N/A \\
\hline$B i+3$ & $1.90 E+01$ & $1.86 E+01$ & & & & & & \\
\hline $\mathrm{Ca}+2$ & $4.88 E-01$ & $4.78 \mathrm{E}-01$ & $3.08 \mathrm{E}-01$ & $1.97 \mathrm{E}-01$ & $3.07 \mathrm{E}-01$ & $1.57 \mathrm{E}-01$ & $1.76 \mathrm{E}-01$ & $1.19 E-01$ \\
\hline $\mathrm{Cd}+2$ & N/A & N/A & N/A & $\mathrm{N} / \mathrm{A}$ & IN/A & N/A & N/A & N/A \\
\hline $\mathrm{Ce}+3$ & & & & $7.71 E+00$ & & $4.48 \mathrm{E}-04$ & $6.33 E+00$ & $1.79 \mathrm{E}+02$ \\
\hline Cl- & $2.01 E+02$ & $1.97 E+02$ & $1.13 E+02$ & $2.12 \mathrm{E}+01$ & 3.3:3E + 01 & $1.61 E+01$ & $1.80 E+01$ & $1.22 \mathrm{E}+01$ \\
\hline $\mathrm{Co}+3$ & N/A & $\mathrm{N} / \mathrm{A}$ & N/A & N/A & $I N / A$ & N/A & N/A & N/A \\
\hline $\cos -2$ & $7.52 E+0.2$ & $7.38 E+02$ & $3.46 E+03$ & $3.51 E+03$ & $5.43 \mathrm{E}+03$ & $4.01 E+03$ & $4.49 E+03$ & $3.03 E+03$ \\
\hline $\mathrm{Cr}(\mathrm{OH}) 4$ & $1.10 E+01$ & $1.08 E+01$ & $3.52 E+00$ & $5.16 E+00$ & $8.03 E+00$ & $4.99 \mathrm{E}+0 \mathrm{O}$ & $5.59 \mathrm{E}+00$ & $3.77 \mathrm{E}+00$ \\
\hline Cs + & & & & $4.71 E+00$ & & $1.13 E+01$ & $2.69 E+00$ & $1.00 E+02$ \\
\hline $\mathrm{Cu}+2$ & N/A & N/A & $\mathrm{N} / \mathrm{A}$ & N/A & IN/A & N/A & N/A & $\mathrm{N} / \mathrm{A}$ \\
\hline F- & $1.56 E+04$ & $1.53 E+04$ & & & & & & \\
\hline $\mathrm{Fe}+3$ & $2.51 E+01$ & $2,46 \mathrm{E}+01$ & $1.76 E+01$ & $9.10 E+00$ & 1.4KE +01 & $6.72 \mathrm{E}+00$ & $7.53 E+00$ & $5.08 E+00$ \\
\hline $\mathrm{H} 2 \mathrm{O}$ & $3.16 E+05$ & $3.10 E+05$ & $6.71 \mathrm{E}+05$ & $4.79 E+05$ & $7.31 E+05$ & $4.32 E+05$ & $4.92 \mathrm{E}+05$ & $5.44 E+05$ \\
\hline $\mathrm{Hg}+2$ & $N / A$ & N/A & N/A & $N / A$ & $\mathrm{~N} / \mathrm{A}$ & N/A & N/A & N/A \\
\hline $\mathrm{K}+$ & 4.27E + 02 & $4.19 E+02$ & & & & & & \\
\hline $\mathrm{La}+3$ & $2.92 \mathrm{E}+00$ & $2.86 E+00$ & & & & & & \\
\hline $\mathrm{Mg}+2$ & $N / A$ & N/A & N/A & N/A & $\mathrm{N} / \mathrm{A}$ & N/A & N/A & N/A \\
\hline $\mathrm{Mn}+4$ & $5.69 \mathrm{E}-02$ & $5.59 E-02$ & & & & & & \\
\hline $\mathrm{Mo}+6$ & N/A & N/A & N/A & N/A & $\mathrm{N} / \mathrm{A}$ & N/A & N/A & N/A \\
\hline $\mathrm{Na}+$ & $3.84 \mathrm{E}+04$ & $3.77 \mathrm{E}+04$ & $3.06 \mathrm{E}+04$ & $2.86 \mathrm{E}+0.4$ & $4.4 ! E+04$ & $3.23 E+04$ & $3.62 E+04$ & $2.44 \mathrm{E}+04$ \\
\hline $\mathrm{Ni}+3$ & $7.51 \mathrm{E}-01$ & 7.37E-01 & $1.26 \mathrm{E}+00$ & $2.82 \mathrm{E}-01$ & $4.42 E-01$ & $2.13 \mathrm{E}-01$ & $2.39 \mathrm{E}-01$ & $1.61 \mathrm{E}-01$ \\
\hline No2- & & & $2.12 \mathrm{E}+01$ & $2.19 E+02$ & $3.51 E+02$ & & & \\
\hline NO3- & $3.79 E+04$ & $3.72 \mathrm{E}+04$ & $9.65 \mathrm{E}+03$ & $4.11 \mathrm{E}+03$ & $6.45 \mathrm{E}+03$ & $2.96 E+03$ & $3.32 \mathrm{E}+03$ & $2.24 \mathrm{E}+03$ \\
\hline $\mathrm{OH}-$ & $2.68 E+03$ & $2.63 E+03$ & $1.70 \mathrm{E}+04$ & $1.63 E+04$ & 2.5:E+04 & $1.91 E+04$ & $2.14 \mathrm{E}+04$ & $1.45 \mathrm{E}+04$ \\
\hline \multicolumn{9}{|l|}{$\mathrm{Pb}+4$} \\
\hline PO4-3 & $1.38 E+03$ & $1.35 E+03$ & $2.41 \mathrm{E}+03$ & $2.88 \mathrm{E}+03$ & $4,45 E+03$ & $3.38 \mathrm{E}+03$ & $3.78 E+03$ & $2.56 E+03$ \\
\hline $\mathbf{R b}+$ & N/A & $N / A$ & N/A & N/A & N/A & N/A & N/A & N/A \\
\hline $\mathrm{Re}+7$ & $\mathrm{~N} / \mathrm{A}$ & N/A & $N / A$ & N/A & $M / A$ & $\mathrm{~N} / \mathrm{A}$ & $\mathrm{N} / \mathrm{A}$ & N/A \\
\hline $\mathrm{Rh}+\mathbf{3}$ & $\mathrm{N} / \mathrm{A}$ & $N / A$ & $\mathrm{~N} / \mathrm{A}$ & $\mathrm{N} / \mathrm{A}$ & N/A & N/A & N/A & $N / A$ \\
\hline$R u+3$ & N/A & $N / A$ & $N / A$ & $N / A$ & $\mathrm{~N} / \mathrm{A}$ & $\mathrm{N} / \mathrm{A}$ & N/A & N/A \\
\hline$S_{\theta}+6$ & N/A & N/A & $N / A$ & N/A & N/A & N/A & N/A & N/A \\
\hline \multicolumn{9}{|l|}{$\mathrm{Si}+4$} \\
\hline so4-2 & $2.30 E+01$ & $2.26 E+01$ & $3.73 E+03$ & $4.31 E+03$ & $6.66 \mathrm{E}+\mathrm{O} 3$ & $5.05 E+03$ & $5.66 E+03$ & $3.82 E+03$ \\
\hline $\mathrm{S} r+2$ & $5.55 E+01$ & $5.44 E+01$ & & $6.31 \mathrm{E}-03$ & 1.2:3E-02 & $9.26 \mathrm{E}-04$ & $1.99 \mathrm{E}-01$ & $8.14 \mathrm{E}-02$ \\
\hline Te+6 & N/A & $\mathrm{N} / \mathrm{A}$ & $N / A$ & N/A & $\mathrm{A} / \mathrm{A}$ & N/A & $\mathrm{N} / \mathrm{A}$ & $N / A$ \\
\hline$T h+4$ & N/A & $N / A$ & N/A & N/A & $N / A$ & N/A & $N / A$ & N/A \\
\hline$T I+4$ & N/A & N/A & N/A & N/A & AI/A & N/A & N/A & N/A \\
\hline TOC & $1.36 \mathrm{E}+03$ & $1.34 E+03$ & $2.76 \mathrm{E}+02$ & & & & & \\
\hline Uo2 +2 & & & $2.55 E+03$ & $2.01 E+03$ & $3.10 E+03$ & $2.29 E+03$ & $2.56 \mathrm{E}+03$ & $1.73 E+03$ \\
\hline$Z n+2$ & N/A & $\mathrm{N} / \mathrm{A}$ & N/A & N/A & $\mathrm{N} / \mathrm{A}$ & N/A & N/A & N/A \\
\hline \multicolumn{9}{|l|}{$\mathrm{ZrO} 2: 2 \mathrm{H} 2 \mathrm{O}$} \\
\hline Total & $4.15 \mathrm{E}+05$ & $4.07 E+05$ & $7.41 E+05$ & $5.42 E+05$ & 8.29E + 05 & $5.02 E+05$ & $5.69 E+05$ & $5.97 E+05$ \\
\hline
\end{tabular}


Table A-1. Chemical Inventory Estimates for Single-Shell Tanks

\begin{tabular}{|c|c|c|c|c|c|c|c|c|}
\hline $\begin{array}{c}\text { Soluble } \\
(\mathrm{kg})\end{array}$ & BX-107 & $\mathrm{BX}-108$ & $B \times-109$ & $B \times-110$ & $B x-111$ & $\mathrm{BX}-112$ & BY-101 & BY-102 \\
\hline $\mathrm{Ag}+$ & N/A & N/A & N/A & N/A & N/A & $N / A$ & N/A & $N / A$ \\
\hline $\mathrm{Al}(\mathrm{OH}) 4$ & $1.45 E+04$ & $1.10 \mathrm{E}+03$ & $6.74 \mathrm{E}+03$ & $8.82 E+03$ & $1.11 E+04$ & $5.55 \mathrm{E}+03$ & $1.89 E+04$ & $1.69 \mathrm{E}+04$ \\
\hline$A s+5$ & N/A & N/A & N/A & N/A & N/A & N/A & N/A & $\mathrm{N} / \mathrm{A}$ \\
\hline$B+3$ & N/A & $N / A$ & N/A & N/A & V/A & $\mathbf{N} / \mathbf{A}$ & N/A & N/A \\
\hline $\mathrm{Ba}+2$ & N/A & N/A & N/A & N/A & $\mathbf{W} / \mathrm{A}$ & N/A & N/A & $N / A$ \\
\hline $\mathrm{Be}+2$ & N/A & N/A & N/A & N/A & IV/A & N/A & $N / A$ & N/A \\
\hline$B i+3$ & $9.94 E+01$ & $7.52 E+00$ & $4.62 \mathrm{E}+01$ & $5.58 \mathrm{E}+01$ & $5.60 E+01$ & $9.87 \mathrm{E}+01$ & $9.10 E+01$ & $8.12 E+01$ \\
\hline $\mathrm{Ca}+2$ & $1.22 \mathrm{E}+00$ & $9.23 E-02$ & $1.09 E+01$ & $1.70 E+01$ & $7.07 \mathrm{E}+01$ & $1.36 \mathrm{E}+01$ & $1.37 \mathrm{E}+02$ & $1.23 E+02$ \\
\hline $\mathrm{Cd}+2$ & N/A & N/A & N/A & N/A & IN/A & N/A & N/A & $\mathrm{N} / \mathrm{A}$ \\
\hline $\mathrm{Ce}+3$ & $3.23 \mathrm{E}+01$ & $7.13 \mathrm{E}-02$ & $4.73 \mathrm{E}-03$ & $1.98 \mathrm{E}+01$ & $2.03 \mathrm{E}+01$ & $1.56 E+01$ & $2.61 \mathrm{E}+01$ & $7.55 E+00$ \\
\hline $\mathrm{Cl}-$ & $6.13 E+02$ & $4.64 \mathrm{E}+01$ & $8.73 E+03$ & $8.61 \mathrm{E}+02$ & $2.513 E+03$ & $5.48 \mathrm{E}+02$ & $4.88 \mathrm{E}+03$ & $4.36 \mathrm{E}+0.3$ \\
\hline $\mathrm{Co}+3$ & N/A & N/A & N/A & N/A & IN/A & $\mathrm{N} / \mathrm{A}$ & N/A & $N / A$ \\
\hline $\mathrm{CO} 3-2$ & $1.88 \mathrm{E}+03$ & $1.42 E+02$ & $2.90 E+04$ & $2.53 \mathrm{E}+\mathrm{O} 3$ & $7.40 \mathrm{E}+03$ & $1.75 E+03$ & $1.77 E+04$ & $1.43 E+04$ \\
\hline $\mathrm{Cr}(\mathrm{OH}) 4$ & $6.74 E+01$ & $5.10 E+00$ & $1.57 E+02$ & $5.54 \mathrm{E}+02$ & $2.255+03$ & $9.00 E+01$ & 4.37E + 03 & $3.90 E+03$ \\
\hline $\mathrm{Cs}+$ & $2.47 \mathrm{E}-01$ & 8.09E-03 & 2.79E-01 & $6.63 \mathrm{E}+00$ & $6.82 \mathrm{E}+00$ & $1.23 \mathrm{E}-01$ & $8.74 E+00$ & $2.53 E+00$ \\
\hline $\mathrm{Cu}+2$ & N/A & N/A & N/A & N/A & IN/A & N/A & N/A & N/A \\
\hline $\mathrm{F}-$ & $1.43 E+04$ & $1.09 E+03$ & $6.66 \mathrm{E}+03$ & $7.05 E+03$ & $3.7 \mathrm{~V}+03$ & $8.31 E+03$ & $4.74 \mathrm{E}+03$ & $4.23 E+03$ \\
\hline $\mathrm{Fe}+3$ & $8.82 E+01$ & $6.67 \mathrm{E}+00$ & $9.75 \mathrm{E}+\mathrm{O} 2$ & $8.53 E+01$ & $2.03 E+02$ & $6.96 \mathrm{E}+01$ & $3.85 \mathrm{E}+02$ & $3.41 E+02$ \\
\hline $\mathrm{H} 2 \mathrm{O}$ & $2.51 E+06$ & $1.90 \mathrm{E}+05$ & $5.58 \mathrm{E}+06$ & $1.79 \mathrm{E}+06$ & $3.04 \mathrm{E}+06$ & $1.40 E+06$ & $5.71 E+06$ & $4.99 E+06$ \\
\hline $\mathrm{Hg}+2$ & $\mathrm{~N} / \mathrm{A}$ & N/A & N/A & N/A & IS/A & $N / A$ & N/A & N/A \\
\hline $\mathrm{K}+$ & & & & $8.95 E+01$ & $3.84 E+02$ & & $7.47 E+02$ & $6.67 E+02$ \\
\hline \multicolumn{9}{|l|}{$\mathrm{La}+3$} \\
\hline $\mathrm{Mg}+2$ & N/A & $\mathrm{N} / \mathrm{A}$ & N/A & N/A & IN/A & N/A & N/A & N/A \\
\hline \multicolumn{9}{|l|}{$\mathrm{Mn}+4$} \\
\hline $\mathrm{Mo}+6$ & N/A & N/A & N/A & N/A & N/A & N/A & $N / A$ & N/A \\
\hline $\mathrm{Na}+$ & $2.87 E+05$ & $2.17 \mathrm{E}+04$ & $6.97 \mathrm{E}+05$ & $2.18 \mathrm{E}+0.5$ & $4.03 E+05$ & $1.63 \mathrm{E}+05$ & $7.62 \mathrm{E}+05$ & $6.69 E+05$ \\
\hline $\mathrm{Ni}+3$ & $1.59 E+00$ & $1.20 E-01$ & $1.62 \mathrm{E}+01$ & $1.10 E+01$ & 4.4:E + 01 & $8.70 E+O 0$ & $8.59 E+01$ & $7.66 \mathrm{E}+01$ \\
\hline NO2- & $8.08 E+03$ & $6.11 \mathrm{E}+02$ & $3.75 E+03$ & $1.37 E+04$ & $4.3 \& \mathrm{E}+04$ & $4.34 E+03$ & $8.38 E+04$ & $7.48 E+04$ \\
\hline NO3:- & $7.47 \mathrm{E}+04$ & $5.66 \mathrm{E}+03$ & $8.76 \mathrm{E}+05$ & $2.17 E+05$ & $7.91 E+05$ & $7.96 \mathrm{E}+04$ & $1.53 E+06$ & $1.36 E+06$ \\
\hline $\mathrm{OH}-$ & $1.37 E+05$ & $1.04 E+04$ & $1.94 \mathrm{E}+05$ & $7.02 \mathrm{E}+04$ & 4.7 $\epsilon_{\mathrm{E}}+04$ & $6.45 E+04$ & $8.49 E+04$ & $6.88 E+04$ \\
\hline $\mathrm{Pb}+4$ & & & & $1.13 E+01$ & $4.85 E+01$ & & $9.43 E+01$ & $8.42 E+01$ \\
\hline PO4-3 & $8.42 E+04$ & $6.37 E+03$ & $5.13 E+04$ & $4.02 E+04$ & $1.70 E+04$ & $5.22 E+04$ & $2.08 E+04$ & $1.74 \mathrm{E}+04$ \\
\hline$R b+$ & N/A & N/A & N/A & N/A & $\mathrm{N} / \mathrm{A}$ & $N / A$ & N/A & N/A \\
\hline$R_{\theta}+7$ & N/A & N/A & N/A & N/A & $N / A$ & $N / A$ & $N / A$ & $\mathrm{~N} / \mathrm{A}$ \\
\hline$R h+3$ & N/A & N/A & N/A & N/A & $\mathrm{N} / \mathrm{A}$ & N/A & N/A & N/A \\
\hline $\mathrm{Ru}+3$ & N/A & N/A & N/A & N/A & $\mathrm{N} / \mathrm{A}$ & N/A & N/A & N/A \\
\hline$S e+6$ & N/A & N/A & $\mathrm{N} / \mathrm{A}$ & N/A & $\mathrm{N} / \mathrm{A}$ & N/A & N/A & N/A \\
\hline \multicolumn{9}{|l|}{$\mathrm{Si}+4$} \\
\hline so4-2 & $2.00 E+03$ & $1.52 \mathrm{E}+02$ & $1.01 E+05$ & $4.16 E+03$ & $1.42 \mathrm{E}+04$ & $3.54 \mathrm{E}+03$ & $3.17 E+04$ & $2.65 E+04$ \\
\hline $\mathrm{Sr}+2$ & $2.20 \mathrm{E}-03$ & $2.41 \mathrm{E}-04$ & $1.94 \mathrm{E}-02$ & $1.08 \mathrm{E}-02$ & $1.03 \mathrm{E}-02$ & $2.20 \mathrm{E}-04$ & $1.69 \mathrm{E}-02$ & $6.92 \mathrm{E}-03$ \\
\hline$T a+6$ & N/A & N/A & N/A & N/A & N/A & $N / A$ & $N / A$ & $N / A$ \\
\hline$T h+4$ & N/A & $\mathrm{N} / \mathrm{A}$ & N/A & $N / A$ & $\mathrm{~A} / \mathrm{A}$ & N/A & N/A & $N / A$ \\
\hline $\mathrm{TI}+4$ & $N / A$ & N/A & N/A & N/A & $\mathrm{N} / \mathrm{A}$ & N/A & N/A & N/A \\
\hline TOC & & & & $4.10 E+02$ & $1.98 E+03$ & & $3.93 E+03$ & $3.52 \mathrm{E}+03$ \\
\hline uo2 +2 & $8.07 E+00$ & $6.11 \mathrm{E}-01$ & $1.21 E+03$ & $6.27 E+01$ & $2.54 E+02$ & $6.15 E+00$ & $2.55 \mathrm{E}+03$ & $1.44 E+03$ \\
\hline$Z n+2$ & N/A & $N / A$ & N/A & N/A & N/A & N/A & N/A & N/A \\
\hline $\mathrm{ZrO} 2: 2 \mathrm{H} 2 \mathrm{O}$ & $1.10 E+02$ & $8.36 \mathrm{E}+00$ & $5.13 \mathrm{E}+01$ & $4.99 \mathrm{E}+01$ & $1.03 E+01$ & $4.24 E+01$ & & \\
\hline Total & $3.13 E+06$ & $2.37 E+05$ & $7.56 \varepsilon+06$ & $2.37 E+06$ & $4.38 \mathrm{E}+06$ & $1.78 \mathrm{E}+06$ & $8.28 E+06$ & $7.26 E+06$ \\
\hline
\end{tabular}


Table A-1. Chemical Inventory Estimates for Single-Shell Tanks

\begin{tabular}{|c|c|c|c|c|c|c|c|c|}
\hline $\begin{array}{c}\text { Soluble } \\
(\mathrm{kg})\end{array}$ & BY- 103 & BY-104 & BY-105 & BY-106 & BY $y-107$ & BY-108 & BY-109 & BY-110 \\
\hline $\mathbf{A g}+$ & N/A & N/A & N/A & N/A & N/A & N/A & $\mathrm{N} / \mathrm{A}$ & N/A \\
\hline Al(OH)4 & $2.31 E+04$ & $9.38 \mathrm{E}+03$ & $1.40 \mathrm{E}+04$ & $2.67 E+04$ & $5.99 \mathrm{E}+03$ & & $2.09 \mathrm{E}+04$ & $9.06 \mathrm{E}+\mathrm{O} 3$ \\
\hline As + 5 & N/A & N/A & N/A & $N / A$ & $\mathrm{~N} / \mathrm{A}$ & N/A & N/A & N/A \\
\hline$B+3$ & N/A & N/A & N/A & N/A & N/A & N/A & N/A & N/A \\
\hline $\mathrm{Ba}+2$ & $\mathrm{~N} / \mathrm{A}$ & N/A & N/A & N/A & N/A & $N / A$ & $\mathbf{N} / \mathbf{A}$ & $\mathrm{N} / \mathrm{A}$ \\
\hline $\mathrm{Be}+2$ & $\mathrm{~N} / \mathrm{A}$ & N/A & N/A & N/A & N/A & $N / A$ & N/A & N/A \\
\hline $\mathrm{Bi}+3$ & $1.02 E+02$ & $2.35 E+02$ & $2.48 \mathrm{E}+02$ & $2.43 E+02$ & $1.1 \% E+O 2$ & $1.85 \mathrm{E}+02$ & $1.01 E+02$ & $2.20 \mathrm{E}+02$ \\
\hline $\mathrm{Ca}+2$ & $1.54 E+02$ & $1.02 E+02$ & $1.34 E+02$ & $2.15 E+02$ & $5.9: 9 E+01$ & $2.67 \mathrm{E}+01$ & $1.52 \mathrm{E}+02$ & $8.37 E+01$ \\
\hline $\mathrm{Cd}+2$ & N/A & $\mathrm{N} / \mathrm{A}$ & N/A & N/A & N/A & N/A & N/A & N/A \\
\hline $\mathrm{Ce}+3$ & $1.35 \mathrm{E}+00$ & $2.66 \mathrm{E}+01$ & $2.07 E+01$ & $2.64 \mathrm{E}+01$ & $9.615 \mathrm{E}+00$ & $1.50 \mathrm{E}+01$ & $8.46 E+00$ & $2.36 \mathrm{E}+01$ \\
\hline $\mathrm{Cl}-$ & $5.47 E+03$ & $4.42 E+03$ & $5.52 \mathrm{E}+03$ & $8.18 \mathrm{E}+03$ & $3.01 E+03$ & $1.93 E+03$ & $5.41 \mathrm{E}+03$ & $3.96 \mathrm{E}+03$ \\
\hline $\mathrm{Co}+3$ & N/A & $N / A$ & $\mathrm{~N} / \mathrm{A}$ & N/A & $\mathbf{N} / \mathbf{A}$ & N/A & $N / A$ & N/A \\
\hline $\cos -2$ & $1.59 \mathrm{E}+04$ & $1.31 E+04$ & $1.78 E+04$ & $2.36 \varepsilon+04$ & $8.65 \mathrm{E}+03$ & $5.42 E+03$ & $1.91 E+04$ & $1.12 \mathrm{E}+04$ \\
\hline $\mathrm{Cr}(\mathrm{OH}) 4$ & $4.89 E+03$ & $3.21 E+03$ & $4.23 E+03$ & $8.80 E+03$ & $1.83 E+03$ & $8.08 \mathrm{E}+02$ & $4.84 E+03$ & $2.63 \mathrm{E}+0.3$ \\
\hline $\mathrm{Cs}+$ & $4.20 \mathrm{E}-01$ & $8.91 E+00$ & $6.92 \mathrm{E}+00$ & $8.84 E+00$ & $3.23 E+00$ & $4.18 \mathrm{E}+00$ & $2.82 \mathrm{E}+00$ & $7.91 \mathrm{E}+00$ \\
\hline $\mathrm{Cu}+2$ & N/A & $\mathrm{N} / \mathrm{A}$ & $N / A$ & N/A & IV/A & $N / A$ & $N / A$ & $N / A$ \\
\hline F. & $5.30 E+03$ & $1.14 E+04$ & $1.21 E+04$ & $1.23 E+04$ & $6.03 \mathrm{E}+03$ & $9.09 E+03$ & $5.25 E+03$ & $1.20 E+04$ \\
\hline $\mathrm{Fe}+3$ & $4.30 E+02$ & $4.09 E+02$ & $4.93 \varepsilon+02$ & $6.71 E+02$ & $2.69 E+02$ & $2.04 \mathrm{E}+02$ & $4.26 \mathrm{E}+02$ & $3.61 E+02$ \\
\hline $\mathrm{H} 2 \mathrm{O}$ & $6.12 \mathrm{E}+06$ & $5.36 \mathrm{E}+06$ & $6.67 \mathrm{E}+06$ & $9.34 E+06$ & $3.24 \mathrm{E}+06$ & $2.47 E+06$ & $6.29 E+06$ & $4.86 E+06$ \\
\hline $\mathrm{Hg}+2$ & $N / A$ & N/A & $\mathrm{N} / \mathrm{A}$ & N/A & IN/A & N/A & N/A & N/A \\
\hline $\mathrm{K}+$ & $8.36 E+02$ & $5.47 E+02$ & $7.21 \mathrm{E}+02$ & $1.16 \mathrm{E}+03$ & $3.18 \mathrm{E}+02$ & $1.35 E+02$ & $8.28 \mathrm{E}+02$ & $4.45 E+02$ \\
\hline \multicolumn{9}{|l|}{$\mathrm{La}+3$} \\
\hline $\mathrm{Mg}+2$ & $N / A$ & N/A & N/A & N/A & IN/A & N/A & $N / A$ & $\mathrm{~N} / \mathrm{A}$ \\
\hline \multicolumn{9}{|l|}{$M n+4$} \\
\hline $\mathrm{Mo}+6$ & $\mathrm{~N} / \mathrm{A}$ & N/A & N/A & N/A & N/A & N/A & N/A & N/A \\
\hline $\mathrm{Na}+$ & $8.22 E+05$ & $7.00 E+05$ & $8.75 E+05$ & $1.24 \mathrm{E}+06$ & $4.25 E+05$ & $3.11 E+05$ & $8.41 E+05$ & $6.30 E+05$ \\
\hline $\mathrm{Ni}+3$ & $9.61 \mathrm{E}+01$ & $6.67 \mathrm{E}+01$ & $8.66 \mathrm{E}+01$ & $1.36 \mathrm{E}+02$ & $4.0 \% E+01$ & $2.08 \mathrm{E}+01$ & $9.52 \mathrm{E}+01$ & $5.62 \mathrm{E}+01$ \\
\hline NO2- & $9.42 \mathrm{E}+04$ & $6.14 E+04$ & $8.09 E+04$ & $1.30 \mathrm{E}+05$ & $3.5 \% E+04$ & $1.51 \mathrm{E}+04$ & $9.28 \mathrm{E}+04$ & $5.08 E+04$ \\
\hline No3- & $1.71 E+06$ & $1.21 E+06$ & $1.56 \mathrm{E}+06$ & $2.44 \mathrm{E}+06$ & $7.5^{\wedge} \mid E+05$ & $3.95 \mathrm{E}+05$ & $1.69 E+06$ & $1.03 E+06$ \\
\hline $\mathrm{OH}-$ & $7.58 E+04$ & $1.21 E+05$ & $1.40 \mathrm{E}+05$ & $1.49 \mathrm{E}+0.5$ & $7.02 \mathrm{E}+04$ & $8.75 E+04$ & $9.18 E+04$ & $1.22 E+05$ \\
\hline $\mathrm{Pb}+4$ & $1.06 \mathrm{E}+02$ & $6.91 E+01$ & $9.11 E+01$ & $1.47 \mathrm{E}+02$ & $4.02 \mathrm{E}+01$ & $1.70 E+01$ & $1.05 E+02$ & $5.62 \mathrm{E}+01$ \\
\hline PO4-3 & $1.99 \mathrm{E}+04$ & $2.40 \mathrm{E}+04$ & $2.89 E+04$ & $3.44 E+04$ & $1.3 \& E+04$ & $1.45 E+04$ & $2.27 E+04$ & $3.01 E+04$ \\
\hline $\mathrm{Rb}+$ & N/A & N/A & N/A & $N / A$ & IN/A & N/A & $N / A$ & $\mathrm{~N} / \mathrm{A}$ \\
\hline$R \theta+7$ & N/A & $\mathrm{N} / \mathrm{A}$ & $\mathrm{N} / \mathrm{A}$ & $N / A$ & IN/A & $N / A$ & $\mathrm{~N} / \mathrm{A}$ & N/A \\
\hline $\mathrm{Rh}+3$ & $\mathrm{~N} / \mathrm{A}$ & $N / A$ & N/A & N/A & N/A & N/A & N/A & $\mathrm{N} / \mathrm{A}$ \\
\hline $\mathrm{Ru}+3$ & N/A & $\mathrm{N} / \mathrm{A}$ & N/A & N/A & N/A & N/A & N/A & $\mathrm{N} / \mathrm{A}$ \\
\hline $\mathrm{Se}+6$ & N/A & N/A & N/A & N/A & N/A & N/A & N/A & $\mathrm{N} / \mathrm{A}$ \\
\hline Si +4 & & & & & & $2.46 \mathrm{E}+01$ & & \\
\hline SO4-2 & $3.05 E+04$ & $6.27 E+04$ & $6.87 E+04$ & $6.82 E+04$ & $3.65 \mathrm{EE}+04$ & $4.79 E+04$ & $3.46 \mathrm{E}+04$ & $5.60 E+04$ \\
\hline $\mathrm{Sr}+2$ & $3.46 \mathrm{E}-02$ & $1.41 E-02$ & $1.19 \mathrm{gE}-\mathrm{O} 2$ & $1.80 \mathrm{E}-02$ & $7.73 \mathrm{E}-03$ & $2,50 E-03$ & $4.97 E-03$ & $1.65 \mathrm{E}-02$ \\
\hline$T_{e}+6$ & $\mathrm{~N} / \mathrm{A}$ & N/A & N/A & N/A & N/A & N/A & N/A & N/A \\
\hline$T h+4$ & $\mathrm{~N} / \mathrm{A}$ & $\mathrm{N} / \mathrm{A}$ & N/A & N/A & $\mathrm{N} / \mathrm{A}$ & N/A & N/A & N/A \\
\hline $\mathrm{TI}+4$ & N/A & $\mathrm{N} / \mathrm{A}$ & $\mathrm{N} / \mathrm{A}$ & N/A & $\mathrm{N} / \mathrm{A}$ & $\mathrm{N} / \mathrm{A}$ & N/A & N/A \\
\hline TOC & $4.42 E+03$ & $7.48 \mathrm{E}+03$ & $8.15 E+03$ & $1.04 \mathrm{E}+04$ & $5.98 \mathrm{E}+03$ & $8.28 E+03$ & $4.35 E+03$ & $9.46 E+03$ \\
\hline $\mathrm{UO} 2+2$ & $6.54 E+02$ & $1.37 E+03$ & $2.32 E+03$ & $1.38 E+03$ & $9.47 E+02$ & $1.10 E+03$ & $2.55 E+03$ & $1.22 \mathrm{E}+03$ \\
\hline$Z n+2$ & N/A & N/A & $\mathrm{N} / \mathrm{A}$ & $\mathrm{N} / \mathrm{A}$ & $\mathrm{N} / \mathrm{A}$ & N/A & N/A & $\mathbf{N} / \mathbf{A}$ \\
\hline $\mathrm{ZrO} 2: 2 \mathrm{H} 2 \mathrm{O}$ & & & & & $3.21 \mathrm{E}-01$ & & & $1.19 \mathrm{E}+01$ \\
\hline Total & $8.93 E+06$ & $7.59 E+06$ & $9.49 E+06$ & $1.35 E+07$ & $4.61 E+06$ & $3.36 \mathrm{E}+06$ & $9.13 \mathrm{E}+06$ & $6.83 E+06$ \\
\hline
\end{tabular}


Table A-1. Chemical Inventory Estimates for Single-Shell Tanks

\begin{tabular}{|c|c|c|c|c|c|c|c|c|}
\hline $\begin{array}{c}\text { Soluble } \\
(\mathrm{kg})\end{array}$ & BY-111 & $B Y-112$ & C-101 & C- 102 & C-103 & C-104 & C-105 & C- 106 \\
\hline $\mathrm{Ag}+$ & $N / A$ & N/A & N/A & N/A & N/A & N/A & N/A & N/A \\
\hline Al(OH)4- & $2.34 E+04$ & $1.46 \mathrm{E}+04$ & $6.77 E+03$ & $6.97 E+04$ & $6.3 .3 E+03$ & $3.43 E+04$ & $2.49 \mathrm{E}+04$ & $2.48 E+03$ \\
\hline $\mathrm{As}+5$ & N/A & $\mathrm{N} / \mathbf{A}$ & N/A & N/A & N/A & N/A & $N / A$ & $N / A$ \\
\hline$B+3$ & N/A & $\mathrm{N} / \mathrm{A}$ & N/A & N/A & V/A & N/A & $N / A$ & N/A \\
\hline $\mathrm{Ba}+2$ & N/A & $N / A$ & $N / A$ & $N / A$ & $\mathrm{~N} / \mathrm{A}$ & N/A & N/A & N/A \\
\hline $\mathrm{Be}+2$ & N/A & N/A & $N / A$ & N/A & IV/A & $N / A$ & N/A & N/A \\
\hline$B i+3$ & $1.13 E+02$ & $7.81 \mathrm{E}+01$ & & & & & & \\
\hline $\mathrm{C} a+2$ & $1.70 E+02$ & $1.08 E+02$ & $1.16 \mathrm{E}+00$ & $4.84 E+00$ & 7.COE-01 & $3.12 E+0 O$ & $1.30 E+00$ & $2.09 E+00$ \\
\hline $\mathrm{Cd}+2$ & N/A & $\mathrm{N} / \mathrm{A}$ & $N / A$ & N/A & IV/A & N/A & N/A & N/A \\
\hline $\mathrm{C}_{\theta}+3$ & $2.67 E+01$ & $4.89 E+00$ & $1.66 \mathrm{E}-03$ & & & & $7.16 \mathrm{E}-05$ & \\
\hline $\mathrm{Cl}$ & $6.05 E+03$ & $3.91 E+03$ & $6.51 E+02$ & $1.08 E+03$ & $1.113 \mathrm{E}+02$ & $1.45 \mathrm{E}+03$ & $3.36 \mathrm{E}+02$ & $9.29 \mathrm{E}+\mathrm{O} 2$ \\
\hline $\mathrm{Co}+3$ & N/A & N/A & $N / A$ & $N / A$ & IN/A & N/A & N/A & $N / A$ \\
\hline $\cos -2$ & $2.00 E+04$ & $1.19 \mathrm{E}+04$ & $2.75 \mathrm{E}+03$ & $8.38 \mathrm{E}+03$ & $1.013 E+03$ & $6.94 \mathrm{E}+03$ & $2.25 E+03$ & $4.81 E+03$ \\
\hline $\mathrm{Cr}(\mathrm{OH}) 4$ & $5.42 E+03$ & $3.43 E+03$ & $3.52 \mathrm{E}+01$ & $7.75 E+01$ & $1.19 \mathrm{E}+01$ & $4.24 E+01$ & $2.85 E+01$ & $2.99 E+01$ \\
\hline Cs + & $8.94 \mathrm{E}+00$ & $1.64 E+00$ & $3.06 \mathrm{E}+00$ & & & & $4.69 E+00$ & \\
\hline $\mathrm{Cu}+2$ & N/A & N/A & $N / A$ & N/A & IN/A & N/A & N/A & $\mathrm{N} / \mathrm{A}$ \\
\hline F- & $5.87 E+03$ & $4.03 E+03$ & & $1.97 E+04$ & & $4.04 \mathrm{E}+04$ & & \\
\hline $\mathrm{Fo+3}$ & $4.75 \mathrm{E}+02$ & $3.10 E+02$ & $8.94 E+01$ & $2.79 E+02$ & 3.7. $\mathrm{HE}+01$ & $1.74 \mathrm{E}+02$ & $7.48 \mathrm{E}+01$ & $1.31 \mathrm{E}+02$ \\
\hline $\mathrm{H} 2 \mathrm{O}$ & $6.95 \mathrm{E}+06$ & $4.41 \mathrm{E}+06$ & $8.32 E+05$ & $7.96 \mathrm{E}+06$ & $9.71 \mathrm{BE}+05$ & $5.68 \mathrm{E}+06$ & $2.41 E+06$ & $2.68 E+06$ \\
\hline $\mathrm{Hg}+2$ & N/A & N/A & N/A & N/A & IV/A & N/A & N/A & $N / A$ \\
\hline $\mathrm{K}+$ & $9.27 E+02$ & $5.86 E+02$ & & $8.04 E+01$ & & $1.51 E+02$ & & \\
\hline \multicolumn{9}{|l|}{$\mathrm{La}+3$} \\
\hline $\mathrm{Mg}+2$ & N/A & N/A & N/A & N/A & IN/A & N/A & N/A & N/A \\
\hline \multicolumn{9}{|l|}{$\mathrm{Mn}+4$} \\
\hline $\mathrm{Mo}+6$ & N/A & N/A & N/A & N/A & IN/A & $\mathrm{N} / \mathrm{A}$ & N/A & $\mathrm{N} / \mathrm{A}$ \\
\hline $\mathrm{Na}+$ & $9.30 E+05$ & $5.90 E+05$ & $4.79 \mathrm{E}+04$ & $1.49 E+05$ & $1.5 \$ E+04$ & $1.61 E+05$ & $4.96 \mathrm{E}+04$ & $8.83 E+04$ \\
\hline $\mathrm{Ni}+3$ & $1.06 E+02$ & $6.75 \mathrm{E}+01$ & $1.79 E+00$ & $7.67 E+00$ & 1.1: $E+\infty O$ & $4.32 E+00$ & $2.00 \mathrm{E}+00$ & $6.03 \mathrm{E}+00$ \\
\hline NO2- & $1.04 E+05$ & $6.57 E+04$ & $1.49 \varepsilon+03$ & $1.86 E+04$ & $1.6 \% \mathrm{E}+03$ & $1.22 \mathrm{E}+04$ & $1.32 \mathrm{E}+04$ & $4.60 E+03$ \\
\hline NO3- & $1.89 E+06$ & $1.21 \mathrm{E}+06$ & $7.06 E+04$ & $1.84 E+05$ & $1.46 E+04$ & $1.16 E+05$ & $6.22 \mathrm{E}+04$ & $7.78 \mathrm{E}+0.4$ \\
\hline $\mathrm{OH}-$ & $9.61 E+04$ & $5.97 \mathrm{E}+04$ & $9.86 \mathrm{E}+03$ & $1.52 E+04$ & $5.7 \mathrm{E}+03$ & $3.73 E+04$ & $8.29 E+03$ & $4.04 E+04$ \\
\hline $\mathrm{Pb}+4$ & $1.17 \mathrm{E}+02$ & $7.40 E+01$ & & & & $2.70 \mathrm{E}-02$ & & \\
\hline PO4-3 & $2.42 \mathrm{E}+04$ & $1.50 E+04$ & $9.43 \mathrm{E}+02$ & $1.25 E+03$ & $7.41 \mathrm{E}+01$ & $6.22 \mathrm{E}+02$ & $2.44 E+02$ & $7.78 \mathrm{E}+02$ \\
\hline $\mathbf{R b}+$ & N/A & N/A & N/A & $N / A$ & $\mathrm{~N} / \mathrm{A}$ & N/A & $\mathrm{N} / \mathrm{A}$ & N/A \\
\hline$R \theta+7$ & N/A & $N / A$ & N/A & $\mathrm{N} / \mathrm{A}$ & $\mathrm{N} / \mathrm{A}$ & N/A & $N / A$ & N/A \\
\hline $\mathrm{Rh}+3$ & N/A & N/A & N/A & N/A & $\mathrm{N} / \mathrm{A}$ & N/A & N/A & N/A \\
\hline $\mathrm{Ru}+3$ & N/A & N/A & N/A & N/A & $\mathrm{N} / \mathrm{A}$ & N/A & N/A & $\mathrm{N} / \mathrm{A}$ \\
\hline $5 \theta+6$ & N/A & N/A & N/A & N/A & $\mathrm{N} / \mathrm{A}$ & N/A & N/A & N/A \\
\hline \multicolumn{9}{|l|}{$S i+4$} \\
\hline SO4-2 & $3.70 E+04$ & $2,45 E+04$ & $7.20 \mathrm{E}+03$ & $4.69 \mathrm{E}+03$ & $8.57 E+01$ & $9.48 E+02$ & $2.06 E+03$ & $4.42 \mathrm{E}+03$ \\
\hline $\mathrm{sr}+2$ & $1.66 \mathrm{E}-02$ & $3.75 E-03$ & $3.48 \mathrm{E}-03$ & $5.19 \mathrm{E}-02$ & $2.01 \mathrm{E}-\mathrm{O} 2$ & $1.23 \mathrm{E}-01$ & $1.42 \mathrm{E}-01$ & $2.73 \mathrm{E}-02$ \\
\hline$T_{\theta}+6$ & $N / A$ & $N / A$ & N/A & $N / A$ & $\mathrm{~N} / \mathrm{A}$ & N/A & N/A & N/A \\
\hline$T h+4$ & N/A & N/A & $N / A$ & $N / A$ & $\mathbf{N} / \mathbf{A}$ & N/A & N/A & N/A \\
\hline$T+4$ & N/A & N/A & N/A & N/A & $\mathrm{N} / \mathrm{A}$ & N/A & N/A & $N / A$ \\
\hline TOC & $4.89 \mathrm{E}+03$ & $3.28 \mathrm{E}+03$ & & $4.54 \mathrm{E}+00$ & & $1.77 E+04$ & & $8.56 E+02$ \\
\hline $\mathrm{UO} 2+2$ & $2.06 \mathrm{E}+03$ & $8.00 E+02$ & $8.45 \mathrm{E}+02$ & $4.26 \mathrm{E}+03$ & $4.3 \mathrm{C} E+\mathrm{O} 2$ & $2.14 E+03$ & $1.16 E+03$ & $3.70 E+03$ \\
\hline $2 n+2$ & N/A & N/A & $N / A$ & $\mathrm{~N} / \mathrm{A}$ & M/A & N/A & $\mathrm{N} / \mathrm{A}$ & N/A \\
\hline $\mathrm{ZrO} 2: 2 \mathrm{H} 2 \mathrm{O}$ & & & & $9.85 E+02$ & & $2.04 \mathrm{E}+03$ & & \\
\hline Total & $1.01 E+07$ & $6.41 \mathrm{E}+06$ & $9.81 \mathrm{E}+0.5$ & $8.43 E+06$ & $1.02 E+06$ & $6.11 \mathrm{E}+06$ & $2.57 E+06$ & $2.91 \mathrm{E}+06$ \\
\hline
\end{tabular}


Table A-1. Chemical Inventory Estimates for Single-Shell Tanks

\begin{tabular}{|c|c|c|c|c|c|c|c|c|}
\hline $\begin{array}{c}\text { Soluble } \\
(\mathrm{kg})\end{array}$ & $C-107$ & C-108 & C-109 & C-110 & C- 111 & $C-112$ & $C-201$ & C. 202 \\
\hline $\mathrm{Ag}+$ & N/A & N/A & N/A & N/A & N/A & N/A & N/A & N/A \\
\hline Al $\{\mathrm{OH}\}$ 4- & $9.14 E+03$ & $7.59 \mathrm{E}+02$ & $4.22 \mathrm{E}+02$ & $7.88 E+03$ & $4.30 E+03$ & $3.81 E+03$ & & \\
\hline As +5 & N/A & N/A & N/A & N/A & $N / A$ & N/A & N/A & $N / A$ \\
\hline $\mathrm{B}+3$ & N/A & N/A & $N / A$ & N/A & N/A & N/A & N/A & N/A \\
\hline $\mathrm{Ba}+2$ & N/A & $N / A$ & N/A & N/A & N/A & N/A & $N / A$ & N/A \\
\hline $\mathrm{B} \theta+2$ & N/A & N/A & N/A & N/A & $\mathrm{N} / \mathrm{A}$ & N/A & N/A & N/A \\
\hline $\mathrm{Bi}+3$ & $7.09 E+01$ & $5.20 E+00$ & $2.89 E+00$ & $5.40 \mathrm{E}+01$ & $1.04 E+01$ & $4.33 E+O O$ & & \\
\hline $\mathrm{Ca}+2$ & $1.10 \mathrm{E}+\mathrm{OO}$ & $7.96 \mathrm{E}-01$ & $1.65 E+00$ & 6.63E-01 & 3.8SE-01 & $2.75 E+00$ & 7.67E-03 & $3.84 \mathrm{E}-03$ \\
\hline $\mathrm{Cd}+2$ & N/A & N/A & $N / A$ & $N / A$ & N/A & N/A & N/A & N/A \\
\hline $\mathrm{Ce}+3$ & $1.71 \mathrm{E}-01$ & 3.57E-03 & $5.61 \mathrm{E}-01$ & & $3.46 \mathrm{iE}-01$ & $1.57 E+00$ & & \\
\hline $\mathrm{Cl}-$ & $6.86 \mathrm{E}+02$ & $5.64 \mathrm{E}+02$ & $4.08 E+02$ & $3.33 E+02$ & $1.19 \mathrm{E}+\mathrm{O} 2$ & $6.07 \mathrm{E}+02$ & $7.85 \mathrm{E}-01$ & $3.92 \mathrm{E}-01$ \\
\hline $\mathrm{Co}+3$ & $\mathrm{~N} / \mathrm{A}$ & N/A & N/A & N/A & $N / A$ & $N / A$ & N/A & N/A \\
\hline $\mathrm{co3}-2$ & $2.46 E+03$ & $1.96 \mathrm{E}+03$ & $2.55 E+03$ & $1.02 E+03$ & $6.00 E+02$ & $4.26 E+03$ & $1.95 E+02$ & $9.76 \mathrm{E}+01$ \\
\hline $\mathrm{Cr}(\mathrm{OH}) 4$ & $4.81 E+01$ & $1.11 \mathrm{E}+01$ & $3.34 \mathrm{E}+00$ & $3.66 \mathrm{E}+01$ & $1.02 E+01$ & $6.43 E+00$ & 2.43E-01 & $1.22 \mathrm{E}-\mathrm{O} 1$ \\
\hline $\mathrm{Cs}+$ & 1.43E-01 & $7.65 \mathrm{E}-05$ & $9.20 \mathrm{E}-\mathrm{O} 2$ & & $7.14 \mathrm{E}-02$ & $6.17 \mathrm{E}-\mathrm{O} 2$ & 3.09E-05 & \\
\hline $\mathrm{Cu}+2$ & N/A & N/A & N/A & N/A & N/A & N/A & N/A & N/A \\
\hline F. & $1.02 E+04$ & $7.51 \mathrm{E}+02$ & $4.17 \mathrm{E}+\mathrm{O2}$ & $7.79 E+03$ & $1.50 E+03$ & $6.24 \mathrm{E}+02$ & & \\
\hline $\mathrm{Fe}+3$ & $7.45 \mathrm{E}+01$ & $6.15 \mathrm{E}+01$ & $2.31 E+01$ & $4.79 \mathrm{E}+01$ & $1.40 E+01$ & $3.27 E+01$ & $3.28 E-01$ & $1.64 \mathrm{E}-01$ \\
\hline $\mathrm{H} 2 \mathrm{O}$ & $1.93 E+06$ & $4.09 E+05$ & $5.04 E+05$ & $1.36 \mathrm{E}+06$ & $5.04 E+05$ & $8.47 E+05$ & $2.11 \mathrm{E}+04$ & $1.05 E+04$ \\
\hline $\mathrm{Hg}+2$ & N/A & N/A & $N / A$ & N/A & N/A & N/A & N/A & N/A \\
\hline$K+$ & & & $1.75 E+01$ & & & $7.48 E+00$ & & \\
\hline \multicolumn{9}{|l|}{$\mathrm{La}+3$} \\
\hline $\mathrm{Mg}+2$ & N/A & N/A & N/A & N/A & N/A & $\mathrm{N} / \mathrm{A}$ & N/A & $N / A$ \\
\hline \multicolumn{9}{|l|}{$M n+4$} \\
\hline $\mathrm{Mo}+6$ & N/A & $N / A$ & $N / A$ & N/A & N/A & N/A & N/A & N/A \\
\hline $\mathrm{Na}+$ & $2.23 E+05$ & $5.07 E+04$ & $3.66 E+04$ & $1.56 E+05$ & $3.82 \varepsilon+04$ & $5.74 E+04$ & $1.57 E+03$ & $7.87 E+02$ \\
\hline $\mathrm{Ni}+3$ & $1.13 E+00$ & $1.47 \mathrm{E}+00$ & $7.08 E+00$ & $8.62 \mathrm{E}-01$ & $1.04 E+00$ & $1.11 E+01$ & $1.04 \mathrm{E}-02$ & 5.20E-03 \\
\hline NO2- & $5.81 E+03$ & $5.21 E+02$ & $1.71 E+03$ & $4.39 E+03$ & $3.14 E+03$ & $3.33 E+03$ & & \\
\hline NO3- & $5.74 E+04$ & $5.82 \mathrm{E}+04$ & $5.99 E+04$ & $4.06 E+04$ & $1.94 \equiv+04$ & $9.41 E+04$ & $1.44 E+02$ & $7.22 E+01$ \\
\hline $\mathrm{OH}-$ & $1.11 E+05$ & $1.52 \mathrm{E}+04$ & $6.73 E+03$ & $7.47 E+04$ & $1.56 \equiv+04$ & $9.10 E+03$ & $9.31 E+02$ & $4.65 \mathrm{E}+\mathrm{O} 2$ \\
\hline $\mathrm{Pb}+4$ & & & $1.12 \mathrm{E}+03$ & & & $4.78 \mathrm{E}+02$ & & \\
\hline PO4-3 & $6.00 E+04$ & $5.20 \mathrm{E}+03$ & $3.41 E+03$ & $4.57 E+04$ & $8.91 \equiv+03$ & $5.16 \mathrm{E}+03$ & $1.65 \mathrm{E}+02$ & $8.23 E+01$ \\
\hline $\mathrm{Rb}+$ & N/A & N/A & N/A & N/A & N/A & N/A & N/A & N/A \\
\hline$R e+7$ & N/A & N/A & $N / A$ & $N / A$ & N/A & N/A & N/A & N/A \\
\hline$R h+3$ & N/A & N/A & N/A & N/A & $N / A$ & $N / A$ & N/A & N/A \\
\hline$R u+3$ & N/A & N/A & $N / A$ & N/A & N/A & N/A & N/A & N/A \\
\hline $\mathrm{Se}+6$ & N/A & N/A & $N / A$ & N/A & $N / A$ & N/A & N/A & N/A \\
\hline \multicolumn{9}{|l|}{$S i+4$} \\
\hline SO4-2 & $1.79 E+03$ & $6.11 \mathrm{E}+03$ & $2.29 E+02$ & $1.09 E+03$ & $2.27 \equiv+02$ & $2.72 \mathrm{E}+\mathrm{O} 2$ & $2.46 \mathrm{E}+02$ & $1.23 \mathrm{E}+\mathrm{O} 2$ \\
\hline $\mathrm{Sr}+2$ & $4.23 \mathrm{E}-03$ & $1.13 \mathrm{E}-03$ & & $6.16 \mathrm{E}-04$ & $4.51 \mathrm{E}-03$ & $1.89 \mathrm{E}-03$ & & \\
\hline $\mathrm{Te}+6$ & N/A & N/A & N/A & N/A & $N / A$ & $N / A$ & N/A & N/A \\
\hline$T h+4$ & N/A & N/A & N/A & $N / A$ & N/A & N/A & N/A & N/A \\
\hline$T I+4$ & N/A & N/A & N/A & N/A & $N / A$ & N/A & N/A & N/A \\
\hline TOC & $9.39 \mathrm{E}+03$ & $4.09 E+02$ & $6.99 E+03$ & & $6.69 \equiv+02$ & $1.00 E+04$ & & \\
\hline $\mathrm{UO} 2+2$ & $5.75 \mathrm{E}+00$ & $3.89 E+02$ & $6.78 \mathrm{E}+02$ & $4.38 E+\infty$ & $1.62 E+02$ & $1.23 E+03$ & $1.11 \mathrm{E}+02$ & $5.57 \mathrm{E}+01$ \\
\hline $\mathrm{Zn}+2$ & N/A & N/A & N/A & $N / A$ & $N / A$ & N/A & N/A & $N / A$ \\
\hline $\mathrm{ZrO} 2: 2 \mathrm{H} 2 \mathrm{O}$ & $7.88 E+01$ & $5.78 E+00$ & $3.21 E+\infty$ & $6.00 E+01$ & $1.15 \equiv+01$ & $4.81 E+00$ & & \\
\hline Total & $2.42 E+06$ & $5.50 \mathrm{E}+05$ & $6.25 \mathrm{E}+05$ & $1.70 \mathrm{E}+06$ & $5.97 \equiv+05$ & $1.04 \mathrm{E}+05$ & $2.44 E+04$ & $1.22 E+04$ \\
\hline
\end{tabular}


Table A-1. Chemical Inventory Estimates for Single-Shell Tanks

\begin{tabular}{|c|c|c|c|c|c|c|c|c|}
\hline $\begin{array}{c}\text { Soluble } \\
(\mathbf{k g})\end{array}$ & $C-203$ & C-204 & S-101 & S-102 & S-103 & S-104 & S-105 & S- 106 \\
\hline $\mathrm{Ag}+$ & $N / A$ & N/A & $\mathrm{N} / \mathrm{A}$ & N/A & NI/A & N/A & $N / A$ & $N / A$ \\
\hline Al(OH)4- & & & $5.88 E+04$ & $6.65 E+04$ & $2.77 E+04$ & $4.68 \mathrm{E}+04$ & $5.41 E+04$ & $6.66 \mathrm{E}+04$ \\
\hline$A s+5$ & N/A & N/A & N/A & N/A & N/A & N/A & N/A & N/A \\
\hline$B+3$ & $\mathbf{N} / \mathbf{A}$ & N/A & N/A & N/A & $\mathrm{N} / \mathrm{A}$ & $N / A$ & N/A & $\mathrm{N} / \mathrm{A}$ \\
\hline$B a+2$ & $\mathrm{~N} / \mathrm{A}$ & N/A & N/A & $N / A$ & $\mathbf{N} / / \mathbf{A}$ & N/A & $N / A$ & N/A \\
\hline $\mathrm{Be}+2$ & N/A & N/A & N/A & N/A & $\mathrm{N} / \mathrm{A}$ & N/A & $\mathrm{N} / \mathrm{A}$ & N/A \\
\hline $\mathrm{Bi}+3$ & & & $3.92 \mathrm{E}+01$ & $1.16 \mathrm{E}+0.2$ & $4.56 \mathrm{E}+01$ & & $6.97 E+01$ & $7.84 \mathrm{E}+01$ \\
\hline $\mathrm{Ca}+2$ & $1.92 \mathrm{E}-02$ & $1.15 \mathrm{E}-02$ & $3.44 \mathrm{E}+01$ & $7.02 E+01$ & 3.57E + 01 & $1.99 E+00$ & $1.01 E+02$ & $1.21 E+02$ \\
\hline $\mathrm{Cd}+2$ & N/A & N/A & N/A & N/A & $\mathrm{N} / \mathrm{A}$ & N/A & $\mathrm{N} / \mathrm{A}$ & $\mathrm{N} / \mathrm{A}$ \\
\hline $\mathrm{Ce}+3$ & & & $5.94 E+00$ & & & & & $1.50 \mathrm{E}-03$ \\
\hline Cl- & $1.96 \mathrm{E}+00$ & $1.18 \mathrm{E}+00$ & $4.67 \mathrm{E}+03$ & $1.02 \mathrm{E}+04$ & $4.12 \mathrm{E}+03$ & $1.54 E+03$ & $7.14 \mathrm{E}+03$ & $8.08 E+03$ \\
\hline $\mathrm{Co}+3$ & N/A & N/A & $N / A$ & $N / A$ & $N / A$ & $N / A$ & N/A & $N / A$ \\
\hline $\mathrm{CO}-2$ & $4.88 \mathrm{E}+02$ & $2.93 \mathrm{E}+02$ & $1.90 \mathrm{E}+04$ & $4.01 E+04$ & $1.90 \mathrm{E}+04$ & $3.07 E+03$ & $4.88 E+04$ & $5.55 E+04$ \\
\hline $\mathrm{Cr}(\mathrm{OH}) 4$ - & 6.08E-01 & $3.65 \mathrm{E}-01$ & $4.23 \mathrm{E}+0.3$ & $3.25 \mathrm{E}+03$ & $1.92 \mathrm{E}+03$ & $5.79 E+03$ & $4.46 \mathrm{E}+03$ & $4.98 E+03$ \\
\hline $\mathrm{Cs}+$ & & & $1.59 \mathrm{E}+00$ & & & 5.68E-01 & $1.56 \mathrm{E}+00$ & $3.08 \mathrm{E}+00$ \\
\hline $\mathrm{Cu}+2$ & $\mathrm{~N} / \mathrm{A}$ & N/A & $N / A$ & $\mathrm{~N} / \mathrm{A}$ & N/A & N/A & N/A & N/A \\
\hline $\mathrm{F}$ & & & $1.82 \mathrm{E}+03$ & $5.40 E+03$ & $2.12 E+03$ & & $3.24 E+03$ & $3.64 E+03$ \\
\hline $\mathrm{Fe}+3$ & $8.19 E-01$ & $4.91 \mathrm{E}-01$ & $1.46 \mathrm{E}+02$ & $9.26 \mathrm{E}+01$ & $9.74 E+01$ & $1.12 \mathrm{E}+\mathrm{O} 2$ & $2.77 E+02$ & $7.22 E+02$ \\
\hline $\mathrm{H} 2 \mathrm{O}$ & $5.27 E+04$ & $2.08 E+04$ & $3.59 E+06$ & $7.81 \mathrm{E}+06$ & $3.38 \mathrm{E}+06$ & $4.58 E+06$ & $7.13 E+06$ & $8.06 \mathrm{E}+06$ \\
\hline $\mathrm{Hg}+2$ & N/A & N/A & N/A & N/A & N/A & N/A & N/A & N/A \\
\hline$K+$ & & & $7.59 \mathrm{E}+02$ & $2.11 E+03$ & $8.66 E+02$ & & $1.56 \mathrm{E}+03$ & $1.76 \mathrm{E}+03$ \\
\hline \multicolumn{9}{|l|}{$\mathrm{La}+3$} \\
\hline $\mathrm{Mg}+2$ & N/A & N/A & N/A & $\mathrm{N} / \mathrm{A}$ & $\mathrm{N} / \mathrm{A}$ & N/A & N/A & N/A \\
\hline $\mathrm{Mn}+4$ & & & $5.05 E+01$ & $1.40 \mathrm{E}+02$ & $5.76 E+01$ & & $1.04 E+02$ & $1.17 E+02$ \\
\hline $\mathrm{Mo}+6$ & N/A & $\mathrm{N} / \mathrm{A}$ & N/A & $\mathrm{N} / \mathrm{A}$ & $N / A$ & N/A & N/A & N/A \\
\hline $\mathrm{Na}+$ & $3.94 \mathrm{E}+03$ & $2.36 \mathrm{E}+\mathrm{O} 3$ & $4.71 E+05$ & $9.98 E+05$ & $4.34 E+05$ & $1.15 \mathrm{E}+05$ & $9.27 E+05$ & $1.05 E+06$ \\
\hline $\mathrm{Ni}+3$ & $2.60 \mathrm{E}-\mathrm{O} 2$ & $1.56 \mathrm{E}-02$ & $2.20 \mathrm{E}+01$ & $4.10 E+01$ & $2.20 \mathrm{E}+01$ & $2.88 \mathrm{E}+00$ & $6.24 E+01$ & $8.75 E+01$ \\
\hline NO2- & & & $8.06 \mathrm{E}+04$ & $2.18 \mathrm{E}+05$ & $8.62 \equiv+04$ & & 1.37E + 05 & $1.55 E+05$ \\
\hline No3- & $3.61 \mathrm{E}+02$ & $2.17 E+02$ & $7.94 E+05$ & $1.38 \mathrm{E}+06$ & $6.32 E+05$ & $2.59 E+05$ & $1.54 E+06$ & $1.74 \mathrm{E}+06$ \\
\hline $\mathrm{OH}-$ & $2.33 E+03$ & $1.38 E+03$ & $7.86 \varepsilon+04$ & $2.51 E+05$ & $1.03 E+05$ & & $1.83 E+05$ & $2.06 \mathrm{E}+05$ \\
\hline $\mathrm{Pb}+4$ & & & $1.14 \mathrm{E}-01$ & $3.17 \mathrm{E}-01$ & $1.3 \mathrm{CIE}-01$ & & $2.35 \mathrm{E}-01$ & 2.64E-01 \\
\hline $\mathrm{PO}_{4-3}$ & $4.12 E+02$ & $2.47 \mathrm{E}+02$ & $7.06 \mathrm{E}+03$ & $2.06 \mathrm{E}+04$ & $8.161=+03$ & & $1.31 E+04$ & $1.47 E+04$ \\
\hline $\mathbf{R b}+$ & N/A & $\mathrm{N} / \mathrm{A}$ & N/A & N/A & $\mathrm{N} / \mathrm{A}$ & N/A & N/A & $\mathrm{N} / \mathrm{A}$ \\
\hline $\mathrm{Ro}+7$ & $\mathbf{N} / \mathbf{A}$ & N/A & N/A & N/A & N/A & N/A & $\mathrm{N} / \mathrm{A}$ & N/A \\
\hline $\mathbf{R h}+\mathbf{3}$ & N/A & N/A & N/A & $\mathrm{N} / \mathrm{A}$ & $N / A$ & N/A & N/A & $N / A$ \\
\hline$R \mathbf{u}+3$ & $\mathrm{~N} / \mathrm{A}$ & N/A & N/A & N/A & N/A & N/A & N/A & N/A \\
\hline Se +6 & N/A & N/A & N/A & N/A & N/A & N/A & N/A & N/A \\
\hline \multicolumn{9}{|l|}{$5 i+4$} \\
\hline SO4-2 & $6.16 \mathrm{E}+02$ & $3.69 \mathrm{E}+02$ & $6.88 \mathrm{E}+03$ & $1.68 \mathrm{E}+04$ & $7.251 \equiv+03$ & $5.62 \mathrm{E}+02$ & $1.52 E+04$ & $1.71 E+04$ \\
\hline $\mathrm{Sr}+2$ & 3.23E-05 & & 2.55E-01 & $2.70 \mathrm{E}-03$ & & 4.54E-01 & $2.43 \mathrm{E}-03$ & 2.63E-01 \\
\hline $\mathrm{Te}+6$ & N/A & N/A & N/A & N/A & N/A & N/A & N/A & $\mathrm{N} / \mathrm{A}$ \\
\hline Th +4 & $N / A$ & $N / A$ & $N / A$ & $N / A$ & N:A & N/A & N/A & N/A \\
\hline$T I+4$ & N/A & N/A & $N / A$ & N/A & $N: A$ & N/A & N/A & N/A \\
\hline TOC & & & $4.43 E+03$ & $1.39 E+04$ & $5.43 E+03$ & & $8.09 E+03$ & $9.07 E+03$ \\
\hline $\mathrm{UO} 2+2$ & $2.79 \mathrm{E}+0.2$ & $1.47 \mathrm{E}-01$ & $4.45 \mathrm{E}+\mathrm{O} 2$ & $1.61 \mathrm{E}+02$ & 6.17E +01 & $1.09 \mathrm{E}+03$ & $1.16 \mathrm{E}+\mathrm{O} 2$ & $5.58 \mathrm{E}+02$ \\
\hline$Z n+2$ & N/A & N/A & N/A & N/A & $\mathbf{N} \cdot \mathbf{A}$ & N/A & N/A & $\mathrm{N} / \mathrm{A}$ \\
\hline \multicolumn{9}{|l|}{$\mathrm{ZrO} 2: 2 \mathrm{H} 2 \mathrm{O}$} \\
\hline Total & $6.11 E+04$ & $2.57 E+04$ & $5.12 E+06$ & $1.08 E+07$ & 4.71E +06 & $5.02 E+06$ & $1.01 E+07$ & $1.14 E+07$ \\
\hline
\end{tabular}


Table A-1. Chemical Inventory Estimates for Single-Shell Tanks

\begin{tabular}{|c|c|c|c|c|c|c|c|c|}
\hline & & & & & & & & \\
\hline $\begin{array}{c}\text { Soluble } \\
(\mathrm{kg})\end{array}$ & S-107 & S-108 & S-109 & S-110 & $s-111$ & S-112 & sx-101 & sx-102 \\
\hline $\mathrm{Ag}_{\mathbf{g}}+$ & N/A & N/A & N/A & N/A & M/A & N/A & N/A & N/A \\
\hline AllOH 4 - & $4.37 E+04$ & $7.17 E+04$ & $6.82 E+04$ & $5.04 E+04$ & $6.58 \mathrm{E}+04$ & $7.57 E+04$ & $6.66 E+04$ & $6.41 E+04$ \\
\hline As +5 & N/A & N/A & N/A & N/A & B//A & N/A & N/A & N/A \\
\hline $8+3$ & N/A & N/A & N/A & N/A & N/A & N/A & N/A & N/A \\
\hline $8 a+2$ & N/A & N/A & N/A & N/A & N/A & N/A & N/A & N/A \\
\hline $80+2$ & N/A & N/A & N/A & N/A & $N / A$ & N/A & N/A & N/A \\
\hline$B i+3$ & $2.05 \mathrm{E}+01$ & $9.19 E+01$ & $8.52 E+01$ & $4.25 E+01$ & $6.8 E E+01$ & $9.68 E+01$ & $2.38 E+01$ & $7.87 E+01$ \\
\hline$C_{a}+2$ & $4.10 E+01$ & $1.32 E+02$ & $1.27 E+02$ & $6.22 E+01$ & $9.64 E+01$ & $1.39 E+02$ & $2.15 E+01$ & $1.13 E+02$ \\
\hline $\mathrm{Cd}+2$ & N/A & N/A & N/A & N/A & N/A & N/A & N/A & $N / A$ \\
\hline $\mathrm{Ce}+3$ & & $7.27 E+00$ & $1.01 E+01$ & $9.95 \mathrm{E}-01$ & $1.1 C E+\infty$ & $6.88 \mathrm{E}+01$ & & 2.70E-02 \\
\hline Cl- & $3.18 E+03$ & $9.42 E+03$ & $8.73 E+03$ & $4.98 \mathrm{E}+03$ & $7.39 E+03$ & $9.92 E+03$ & $4.04 E+03$ & $8.25 E+03$ \\
\hline $\mathrm{Co}+3$ & N/A & N/A & N/A & N/A & N//A & N/A & N/A & N/A \\
\hline $\cos -2$ & $9.84 E+03$ & $6.43 E+04$ & $5.98 E+04$ & $3.06 E+04$ & $4.85 \mathrm{E}+04$ & $6.78 \varepsilon+04$ & $1.29 \varepsilon+04$ & $5.06 E+04$ \\
\hline $\mathrm{Cr}(\mathrm{OH}) 4$ & $4.56 \mathrm{E}+\mathrm{O} 3$ & $4.51 E+03$ & $5.41 E+03$ & $4.21 E+03$ & $4.73 E+03$ & $5.21 E+03$ & $5.50 E+03$ & $1.56 E+04$ \\
\hline $\mathrm{Cs}+$ & & 4.17E+00 & $2.35 E+01$ & $2.62 E+01$ & $2.41 E+01$ & $8.14 E+01$ & & 7.23E-03 \\
\hline $\mathrm{Cu}+2$ & N/A & N/A & $N / A$ & N/A & $N / / A$ & N/A & N/A & N/A \\
\hline F- & $4.80 E+04$ & $4.27 E+03$ & $3.95 E+03$ & $1.97 E+03$ & $3.18 E+03$ & $4.49 E+03$ & $1.11 E+03$ & $3.65 E+03$ \\
\hline$F_{\theta}+3$ & $1.96 E+02$ & $1.97 E+02$ & $6.32 \mathrm{E}+02$ & $1.98 \mathrm{E}+02$ & $2.23 \mathrm{E}+\mathrm{O} 2$ & $2.79 \mathrm{E}+\mathrm{O} 2$ & $1.42 E+02$ & $7.51 E+02$ \\
\hline $\mathrm{H} 2 \mathrm{O}$ & $3.77 E+06$ & $9.41 E+06$ & $8.73 E+06$ & $4.62 \mathrm{E}+06$ & $7.22 E+06$ & $9.91 E+06$ & $3.77 \mathrm{E}+06$ & $7.69 E+06$ \\
\hline $\mathrm{Hg}+2$ & N/A & N/A & N/A & N/A & $\mathbf{N} / \mathbf{A}$ & N/A & N/A & N/A \\
\hline$k+$ & $5.13 \mathrm{E}+\mathrm{O} 2$ & $2.06 \mathrm{E}+03$ & $1.91 E+03$ & $9.53 E+02$ & $1.54 E+03$ & $2.17 E+03$ & $4.59 \mathrm{E}+\mathrm{O} 2$ & $1.70 E+03$ \\
\hline$L a+3$ & & & & & & & & \\
\hline $\mathrm{Mg}+2$ & N/A & $\mathrm{N} / \mathrm{A}$ & N/A & N/A & N//A & N/A & N/A & N/A \\
\hline$M n+4$ & $1.68 \mathrm{E}+01$ & $1.37 E+02$ & $1.27 E+02$ & $6.34 E+01$ & $1.02 \mathrm{E}+02$ & $1.44 \mathrm{E}+\mathrm{O} 2$ & $3.05 E+01$ & $1.13 E+02$ \\
\hline $\mathrm{Mo}+6$ & N/A & $\mathrm{N} / \mathrm{A}$ & $\mathrm{N} / \mathrm{A}$ & N/A & M//A & N/A & N/A & $\mathrm{N} / \mathrm{A}$ \\
\hline $\mathrm{Na}+$ & $4.40 E+05$ & $1.22 E+06$ & $1.13 E+06$ & $6.06 E+05$ & $9.41 E+05$ & $1.29 E+06$ & $3.57 \mathrm{E}+05$ & $9.99 E+05$ \\
\hline $\mathrm{Ni}+3$ & $2.96 E+01$ & $7.81 E+01$ & $8.39 E+01$ & $3.88 \mathrm{E}+01$ & $5.56 \mathrm{E}+01$ & $8.42 E+01$ & $1.45 E+01$ & $8.55 E+01$ \\
\hline NO2. & $4.18 E+04$ & $1.80 E+05$ & $1.67 E+05$ & $8.32 E+04$ & $1.37 E+05$ & $1.90 E+05$ & $4.52 E+04$ & $1.53 E+05$ \\
\hline NO3- & $8.00 E+05$ & $2.03 E+06$ & $1.88 E+06$ & $1.06 E+06$ & $1.59 E+06$ & $2.13 E+06$ & $7.17 E+05$ & $1.63 E+06$ \\
\hline $\mathrm{OH}-$ & $3.23 E+04$ & $2.40 E+05$ & $2.24 E+05$ & $1.02 E+05$ & $1.76 E+05$ & $2.53 E+05$ & $2.59 E+04$ & $2.01 E+05$ \\
\hline $\mathrm{Pb}+4$ & $3.80 \mathrm{E}-02$ & $3.10 \mathrm{E}-\mathrm{O} 1$ & $2.87 \mathrm{E}-01$ & $1.43 \mathrm{E}-01$ & $2.3 \mid \mathrm{E}-01$ & $3.26 \mathrm{E}-01$ & $6.90 \mathrm{E}-02$ & $2.55 E-01$ \\
\hline PO4-3 & $3.33 E+03$ & $1.73 E+04$ & $1.60 E+04$ & $7.97 E+03$ & $1.29 E+04$ & $1.82 E+04$ & $4.28 \mathrm{E}+03$ & $1.46 E+04$ \\
\hline $\mathbf{R b}+$ & N/A & $\mathrm{N} / \mathrm{A}$ & N/A & $\mathrm{N} / \mathrm{A}$ & $\mathrm{N} / \mathrm{A}$ & N/A & N/A & N/A \\
\hline$R e+7$ & N/A & N/A & N/A & N/A & $\mathrm{A} / \mathrm{A}$ & N/A & N/A & N/A \\
\hline$R h+3$ & N/A & N/A & N/A & $N / A$ & $\mathrm{~N} / \mathrm{A}$ & N/A & N/A & $\mathrm{N} / \mathrm{A}$ \\
\hline$R u+3$ & N/A & $\mathrm{N} / \mathrm{A}$ & N/A & $\mathrm{N} / \mathrm{A}$ & $\mathrm{N} / \mathrm{A}$ & N/A & N/A & N/A \\
\hline Se+6 & N/A & N/A & N/A & N/A & $\mathrm{N} / \mathrm{A}$ & N/A & N/A & N/A \\
\hline$S i+4$ & & & & & & & & \\
\hline so4-2 & $5.07 E+03$ & $2.00 E+04$ & $1.86 E+04$ & $9.49 E+03$ & $1.51 E+04$ & $2.11 E+04$ & $4.61 E+03$ & $1.61 E+04$ \\
\hline $\mathrm{Sr}+2$ & 1.37E-01 & $3.74 \mathrm{E}-03$ & $1.11 \mathrm{E}-01$ & $2.92 \mathrm{E}-02$ & $1.4^{\mid E-01}$ & $9.68 \mathrm{E}-02$ & 3.67E-02 & $1.04 \mathrm{E}-01$ \\
\hline$T e+6$ & N/A & N/A & N/A & N/A & $\mathrm{N} / \mathrm{A}$ & N/A & N/A & $\mathrm{N} / \mathrm{A}$ \\
\hline$T h+4$ & N/A & N/A & $\mathrm{N} / \mathrm{A}$ & N/A & $\mathrm{N} / \mathrm{A}$ & N/A & N/A & $\mathrm{N} / \mathrm{A}$ \\
\hline$\pi+4$ & N/A & N/A & N/A & $\mathrm{N} / \mathrm{A}$ & $\mathrm{N} / \mathrm{A}$ & N/A & N/A & N/A \\
\hline TOC & $1.74 \mathrm{E}+03$ & $1.07 E+04$ & $9.87 E+03$ & $4.74 E+03$ & $7.81 E+03$ & $1.12 \mathrm{E}+04$ & $2.60 \mathrm{E}+03$ & $9.31 E+03$ \\
\hline $\mathrm{UO2}+2$ & $3.53 E+02$ & $1.65 E+02$ & $3.11 E+02$ & $1.49 \mathrm{E}+02$ & $2.86 \mathrm{E}+02$ & $2.08 E+02$ & $2.67 E+02$ & $4.67 \mathrm{E}+02$ \\
\hline$Z n+2$ & N/A & $\mathrm{N} / \mathrm{A}$ & $\mathrm{N} / \mathrm{A}$ & N/A & $\mathrm{N} / \mathrm{A}$ & N/A & N/A & N/A \\
\hline $\mathrm{ZrO2}: 2 \mathrm{H} 2 \mathrm{O}$ & $2.35 E+03$ & & & & & & & \\
\hline$\overline{\text { Total }}$ & $5.20 E+06$ & $1.33 E+07$ & $1.23 E+07$ & $6.59 E+06$ & $1.02 E+07$ & $1.40 E+07$ & $5.02 \mathrm{E}+06$ & $1.09 E+07$ \\
\hline
\end{tabular}


Table A-1. Chemical Inventory Estimates for Single-Shell Tanks

\begin{tabular}{|c|c|c|c|c|c|c|c|c|}
\hline $\begin{array}{c}\text { Soluble } \\
(\mathbf{k g})\end{array}$ & $5 \times-103$ & Sx-104 & sX-105 & sx.106 & sx-107 & $5 x-108$ & $5 \times-109$ & $5 x-110$ \\
\hline $\mathrm{Ag}+$ & N/A & $\mathbf{N} / \mathbf{A}$ & $N / A$ & $N / A$ & N/A & N/A & $\mathrm{N} / \mathrm{A}$ & N/A \\
\hline $\mathrm{Al}(\mathrm{OH}) 4$ & $8.14 E+04$ & $7.90 E+04$ & $8.11 E+04$ & $5.80 E+04$ & $1.49 E+04$ & $1.66 E+04$ & $1.83 E+04$ & $3.84 E+03$ \\
\hline$A s+5$ & N/A & N/A & $N / A$ & $N / A$ & NI/A & N/A & $N / A$ & $\mathrm{~N} / \mathrm{A}$ \\
\hline$B+3$ & N/A & N/A & N/A & N/A & N/A & N/A & N/A & $N / A$ \\
\hline $\mathrm{Ba}+2$ & N/A & N/A & N/A & N/A & NI/A & N/A & $\mathrm{N} / \mathrm{A}$ & N/A \\
\hline $\mathrm{B} \theta+2$ & $N / A$ & N/A & N/A & N/A & $\mathrm{N} / \mathrm{A}$ & N/A & N/A & $\mathrm{N} / \mathrm{A}$ \\
\hline $\mathrm{Bi}+3$ & $8.27 E+01$ & $6.82 E+01$ & 9.57E + 01 & $1.10 E+02$ & & & & \\
\hline $\mathrm{Ca}+2$ & $1.21 E+02$ & $9.98 \mathrm{E}+01$ & $1.42 \mathrm{E}+02$ & $4.87 \mathrm{E}+01$ & 8.37E-01 & $8.91 \mathrm{E}-01$ & $8.54 E+01$ & $1.34 E+01$ \\
\hline $\mathrm{Cd}+2$ & N/A & $\mathrm{N} / \mathrm{A}$ & N/A & N/A & N/A & $\mathrm{N} / \mathrm{A}$ & N/A & $N / A$ \\
\hline $\mathrm{Ce}+3$ & $4.67 E+01$ & $9.65 E+00$ & $1.60 E+01$ & $2.96 \mathrm{E}+01$ & & & & \\
\hline $\mathrm{Cl}-$ & $9.09 E+03$ & $7.92 E+03$ & $1.02 E+04$ & $9.29 E+03$ & $6.69 \mathrm{E}+02$ & $7.31 E+02$ & $1.09 \mathrm{E}+03$ & $3.73 E+02$ \\
\hline $\mathrm{Co}+3$ & N/A & $\mathrm{N} / \mathrm{A}$ & $N / A$ & $N / A$ & A/A & $N / A$ & $\mathrm{~N} / \mathrm{A}$ & $\mathrm{N} / \mathrm{A}$ \\
\hline co3-2 & $5.87 E+04$ & $4.90 E+04$ & $6.66 E+04$ & $3.00 E+04$ & $1.29 E+03$ & $1.38 E+03$ & $5.95 E+03$ & $1.81 \mathrm{E}+03$ \\
\hline $\mathrm{Cr}(\mathrm{OH}) 4$ & $6.76 \mathrm{E}+03$ & $6.60 E+03$ & $8.16 E+03$ & $3.11 E+03$ & $3.92 \mathrm{E}+03$ & $4.06 E+03$ & $8.66 \mathrm{E}+03$ & $4.40 E+03$ \\
\hline$C_{s+}$ & $3.04 E+01$ & $2.07 E+00$ & $4.28 E+00$ & $4.18 E+00$ & & & & \\
\hline $\mathrm{Cu}+2$ & N/A & N/A & N/A & N/A & $N / A$ & N/A & $\mathrm{N} / \mathrm{A}$ & N/A \\
\hline $\mathbf{F}-$ & $3.84 E+03$ & $3.17 E+03$ & $4.44 E+03$ & $5.11 E+03$ & & & & \\
\hline $\mathrm{Fo}+3$ & $3.57 E+02$ & $3.14 \mathrm{E}+02$ & $4.11 E+02$ & $8.75 E+01$ & $5.11 E+01$ & $5.40 E+01$ & $1.69 E+02$ & $6.19 E+01$ \\
\hline $\mathrm{H} 2 \mathrm{O}$ & $8.73 E+06$ & $7.38 \mathrm{E}+0.6$ & $1.00 E+07$ & $6.61 E+06$ & $1.69 E+06$ & $1.84 E+06$ & $4.20 E+06$ & $9.19 E+05$ \\
\hline $\mathrm{Hg}+2$ & $N / A$ & N/A & N/A & $\mathrm{N} / \mathrm{A}$ & $N / A$ & N/A & N/A & N/A \\
\hline $\mathrm{K}+$ & $1.86 \mathrm{E}+03$ & $1.53 E+03$ & $2.10 \mathrm{E}+03$ & $1.90 \mathrm{E}+03$ & & & & \\
\hline \multicolumn{9}{|l|}{$\mathrm{La}+3$} \\
\hline $\mathrm{Mg}+2$ & N/A & N/A & N/A & N/A & N/A & N/A & N/A & N/A \\
\hline $\mathrm{Mn}+4$ & $1.23 E+02$ & $1.02 E+02$ & $1.40 \mathrm{E}+02$ & $1.26 \mathrm{E}+02$ & & & & \\
\hline $\mathrm{Mo}+6$ & N/A & $N / A$ & $N / A$ & $\mathrm{~N} / \mathrm{A}$ & $\mathrm{N} / \mathrm{A}$ & N/A & $\mathrm{N} / \mathrm{A}$ & N/A \\
\hline $\mathrm{Ne}+$ & $1.14 \mathrm{E}+06$ & $9.69 \mathrm{E}+05$ & $1.31 \mathrm{E}+06$ & $8.38 \mathrm{E}+05$ & $4.33 \mathrm{E}+04$ & $4.74 \mathrm{E}+04$ & $5.87 \mathrm{E}+05$ & $1.29 \mathrm{E}+05$ \\
\hline $\mathrm{Ni}+3$ & $7.48 \mathrm{E}+01$ & $6.22 E+01$ & $8.78 \mathrm{E}+01$ & $2.94 E+01$ & $1.21 \mathrm{E}+00$ & $1.28 \mathrm{E}+00$ & 4.15E+01 & $7.35 E+00$ \\
\hline $\mathrm{NO} 2-$ & $1.62 E+05$ & $1.34 E+05$ & $1.87 E+05$ & $2.04 E+05$ & & & & \\
\hline NO3. & $1.95 \mathrm{E}+06$ & $1.69 E+06$ & $2.22 E+06$ & $1.08 \mathrm{E}+06$ & $9.83 \mathrm{E}+04$ & $1.08 \mathrm{E}+05$ & $1.56 \mathrm{E}+06$ & $3.37 E+05$ \\
\hline $\mathrm{OH}-$ & $2.07 E+05$ & $1.64 E+05$ & $2.43 E+05$ & $2.28 \mathrm{E}+05$ & & & & \\
\hline $\mathrm{Pb}+4$ & $2.79 \mathrm{E}-01$ & $2.30 \mathrm{E}-01$ & 3.16E-01 & $2.86 \mathrm{E}-01$ & & & & \\
\hline PO4-3 & $1.55 E+04$ & $1.28 E+04$ & $1.78 \mathrm{E}+04$ & $1.92 \mathrm{E}+04$ & & & & \\
\hline $\mathbf{R b}+$ & N/A & $\mathrm{N} / \mathrm{A}$ & $N / A$ & N/A & $\mathrm{N} / / \mathbf{A}$ & N/A & N/A & $N / A$ \\
\hline $\mathrm{Re}+7$ & N/A & $\mathrm{N} / \mathrm{A}$ & N/A & N/A & $\mathrm{N} / / \mathbf{A}$ & N/A & N/A & $\mathrm{N} / \mathrm{A}$ \\
\hline$R h+3$ & N/A & $\mathrm{N} / \mathrm{A}$ & N/A & $N / A$ & $\Lambda / / A$ & N/A & $\mathrm{N} / \mathrm{A}$ & $\mathrm{N} / \mathrm{A}$ \\
\hline$R u+3$ & N/A & N/A & $\mathrm{N} / \mathrm{A}$ & $\mathrm{N} / \mathrm{A}$ & $\mathrm{N} / / \mathrm{A}$ & N/A & N/A & $\mathrm{N} / \mathrm{A}$ \\
\hline $\mathrm{Se}+6$ & $\mathrm{~N} / \mathrm{A}$ & $\mathrm{N} / \mathrm{A}$ & N/A & N/A & N/A & N/A & N/A & N/A \\
\hline \multicolumn{9}{|l|}{$5 i+4$} \\
\hline $504-2$ & $1.83 E+04$ & $1.52 E+04$ & $2.12 E+04$ & $1.42 E+04$ & $2.39 \mathrm{E}+02$ & $2.62 \mathrm{E}+\mathrm{O} 2$ & $3.82 \mathrm{E}+02$ & $1.15 E+02$ \\
\hline $5 r+2$ & $3.58 \mathrm{E}-02$ & $3.68 \mathrm{E}-03$ & $1.88 \mathrm{E}-02$ & $8.24 \mathrm{E}-03$ & 9.1 SE-02 & 5.70E-02 & $1.71 \mathrm{E}-02$ & $3.74 \mathrm{E}-02$ \\
\hline $\mathrm{Te}+6$ & N/A & $N / A$ & $\mathrm{~N} / \mathrm{A}$ & $N / A$ & $\mathrm{~N} / / \mathrm{A}$ & N/A & N/A & $N / A$ \\
\hline$T h+4$ & N/A & $\mathrm{N} / \mathrm{A}$ & N/A & N/A & $\mathrm{N} / \mathrm{A}$ & $N / A$ & N/A & N/A \\
\hline $\mathrm{TI}+4$ & $N / A$ & $N / A$ & $\mathrm{~N} / \mathrm{A}$ & N/A & $\mathrm{N} / \mathrm{A}$ & N/A & $N / A$ & $\mathrm{~N} / \mathrm{A}$ \\
\hline TOC & $9.38 E+03$ & $7.62 E+03$ & $1.09 E+04$ & $1.32 E+04$ & & & & \\
\hline $\mathrm{UO} 2+2$ & $2.15 E+02$ & $2.31 E+02$ & $3.53 E+02$ & $1.37 E+02$ & $3.82 E+O 2$ & $3.89 E+02$ & $7.00 \mathrm{E}+02$ & $4.87 E+02$ \\
\hline$Z n+2$ & $\mathrm{~N} / \mathrm{A}$ & $\mathrm{N} / \mathrm{A}$ & N/A & N/A & $\mathrm{N} / \mathrm{A}$ & N/A & N/A & $\mathrm{N} / \mathrm{A}$ \\
\hline \multicolumn{9}{|l|}{ ZrO2:2H2O } \\
\hline Total & $1.24 \mathrm{E}+07$ & $1.05 E+07$ & $1.42 E+07$ & $9.11 \mathrm{E}+06$ & $1.85 \mathrm{E}+06$ & $2.01 E+06$ & $6.38 \mathrm{E}+06$ & $1.40 E+06$ \\
\hline
\end{tabular}


WHC-SD-WM-TI-784

Revision 0

Table A-1. Chemical Inventory Estimates for Single-Shell Tanks

\begin{tabular}{|c|c|c|c|c|c|c|c|c|}
\hline $\begin{array}{c}\text { Soluble } \\
\text { (kg) }\end{array}$ & $s x-111$ & $5 x-112$ & $5 x-113$ & $5 x-114$ & sx 115 & $\mathrm{~T}-101$ & $T-102$ & $\mathrm{~T}-103$ \\
\hline $\mathrm{Ag}+$ & N/A & N/A & N/A & N/A & N/A & N/A & $N / A$ & N/A \\
\hline $\mathrm{Al}(\mathrm{OH}) 4$ & $1.19 E+04$ & $1.06 E+04$ & $3.12 E+02$ & $7.05 E+03$ & $7.16 E+02$ & $6.98 E+03$ & 3.37E + 03 & $3.71 \mathrm{E}+03$ \\
\hline As +5 & N/A & N/A & N/A & N/A & $\mathrm{N} / \mathrm{A}$ & N/A & N/A & N/A \\
\hline$B+3$ & N/A & $\mathbf{N} / \mathbf{A}$ & N/A & N/A & N/A & N/A & N/A & N/A \\
\hline $\mathrm{Be}+2$ & N/A & $N / A$ & N/A & N/A & $\mathrm{N} / \mathrm{A}$ & N/A & N/A & N/A \\
\hline $\mathrm{Be}+2$ & N/A & N/A & N/A & N/A & N/A & N/A & N/A & N/A \\
\hline $\mathrm{Bi}+3$ & & & & & & & & $5.85 \mathrm{E} \cdot 03$ \\
\hline $\mathrm{Ca}+2$ & $1.79 E+01$ & $7.99 E+00$ & 9.97E-03 & $7.11 E+01$ & $4.77 E+00$ & 4.47E-01 & 2.19E-01 & $1.74 E+00$ \\
\hline $\mathrm{Cd}+2$ & N/A & $\mathrm{N} / \mathrm{A}$ & $N / A$ & N/A & $N / A$ & N/A & $N / A$ & N/A \\
\hline $\mathrm{Ce}+3$ & & & & & & $8.02 E+00$ & 1.36E-01 & $2.76 \mathrm{E}-03$ \\
\hline $\mathrm{Cl}-$ & $7.31 E+02$ & $5.50 \mathrm{OE}+02$ & $1.13 E+01$ & $7.86 E+02$ & $7.15 E+01$ & $7.24 E+01$ & $2.62 E+01$ & $9.45 E+01$ \\
\hline $\mathrm{Co}+3$ & N/A & N/A & $N / A$ & $N / A$ & N/A & $\mathrm{N} / \mathrm{A}$ & N/A & $N / A$ \\
\hline $\cos -2$ & $2.74 E+03$ & $1.64 \mathrm{E}+03$ & $1.54 E+01$ & $5.44 E+03$ & $3.62 E+02$ & $8.74 E+02$ & $5.20 E+02$ & $5.46 E+02$ \\
\hline $\mathrm{Cr}(\mathrm{OH}) 4$ & $6.30 E+03$ & $3.93 E+03$ & $2.69 E+01$ & $8.96 \mathrm{E}+03$ & $1.42 E+03$ & $6.62 \mathrm{E}+00$ & $3.32 \mathrm{E}+00$ & $1.00 \mathrm{E}+02$ \\
\hline Cs + & & $5.05 \mathrm{E}-02$ & $7.55 E+00$ & & $1.53 E+01$ & 4.54E-01 & $7.69 \mathrm{E}-03$ & $3.69 E-02$ \\
\hline $\mathrm{Cu}+2$ & N/A & N/A & N/A & N/A & $\mathrm{N} / \mathrm{A}$ & $\mathrm{N} / \mathrm{A}$ & N/A & N/A \\
\hline $\mathrm{F}-$ & & & & & & & & $2.58 \mathrm{E}+01$ \\
\hline $\mathrm{Fe}+3$ & $9.29 E+01$ & $5.73 E+01$ & $5.29 \mathrm{E}-01$ & $1.60 E+02$ & $2.80 E+01$ & $2.40 E+01$ & $1.17 E+01$ & $1.58 \mathrm{E}+01$ \\
\hline $\mathrm{H} 2 \mathrm{O}$ & $1.36 E+06$ & $1.02 E+06$ & $2.71 E+04$ & $3.72 E+06$ & $1.83 E+05$ & $7.51 E+05$ & $3.62 E+05$ & $3.15 E+05$ \\
\hline $\mathrm{Hg}+2$ & N/A & N/A & N/A & $N / A$ & N/A & N/A & N/A & N/A \\
\hline \multicolumn{9}{|l|}{$\mathrm{k}+$} \\
\hline \multicolumn{9}{|l|}{$\mathrm{La}+3$} \\
\hline $\mathrm{Mg}+2$ & N/A & N/A & $\mathbf{N} / \mathbf{A}$ & N/A & N/A & N/A & N/A & N/A \\
\hline \multicolumn{9}{|l|}{$\mathrm{Mn}+4$} \\
\hline $\mathrm{Mo}+6$ & N/A & N/A & N/A & N/A & N/A & N/A & N/A & N/A \\
\hline $\mathrm{Ne}+$ & $1.91 E+05$ & $1.02 \mathrm{E}+05$ & $7.49 E+02$ & $5.20 E+05$ & $2.57 E+04$ & $9.89 E+03$ & $5.14 \mathrm{E}+03$ & $1.58 \mathrm{E}+04$ \\
\hline $\mathrm{Ni}+3$ & $1.01 \mathrm{E}+01$ & $4.89 \mathrm{E}+\mathrm{OO}$ & $1.37 \mathrm{E}-02$ & $3.58 E+01$ & $3.65 E+00$ & $7.14 \mathrm{E}-01$ & $3.50 E-01$ & $1.29 E+00$ \\
\hline NO2- & & & & & & $1.54 \mathrm{E}+03$ & $7.42 \mathrm{E}+02$ & $1.83 E+03$ \\
\hline No3. & $4.94 \mathrm{E}+05$ & $2.59 E+05$ & $1.70 E+03$ & $1.39 E+06$ & $6.78 E+04$ & $1.43 E+04$ & $5.55 \mathrm{E}+03$ & $3.37 E+04$ \\
\hline $\mathrm{OH}-$ & & & & & & $8.75 E+02$ & $9.87 \mathrm{E}+02$ & $5.43 E+02$ \\
\hline \multicolumn{9}{|l|}{$\mathrm{Pb}+4$} \\
\hline PO4-3 & & & & & & $1.65 \mathrm{E}+02$ & $1.64 \mathrm{E}+02$ & $1.58 \mathrm{E}+02$ \\
\hline $\mathrm{Rb}+$ & N/A & N/A & N/A & N/A & N/A & N/A & N/A & $N / A$ \\
\hline $\mathrm{Re}+7$ & N/A & $\mathrm{N} / \mathrm{A}$ & $N / A$ & N/A & N/A & N/A & N/A & $N / A$ \\
\hline $\mathrm{Rh}+3$ & $N / A$ & N/A & N/A & N/A & N/A & $\mathrm{N} / \mathrm{A}$ & N/A & N/A \\
\hline$R u+3$ & N/A & N/A & N/A & N/A & N/A & N/A & N/A & $N / A$ \\
\hline $\mathrm{S}_{\theta}+6$ & N/A & $N / A$ & N/A & $\mathrm{N} / \mathrm{A}$ & N/A & N/A & N/A & $N / A$ \\
\hline \multicolumn{9}{|l|}{$\mathrm{Si}+4$} \\
\hline SO4-2 & $2.44 E+02$ & $1.90 \mathrm{OE}+02$ & $4.35 E+00$ & $2.54 \mathrm{E}+02$ & $2.20 \equiv+01$ & $2.62 E+02$ & $2.52 \mathrm{E}+\mathrm{O} 2$ & $3.09 E+02$ \\
\hline$S r+2$ & $5.24 \mathrm{E}-02$ & $6.05 \mathrm{E}-02$ & $1.60 \mathrm{E}-02$ & $5.50 \mathrm{E}-02$ & $3.94 \mathrm{E}-\mathrm{O} 2$ & 2.87E-01 & & $1.07 \mathrm{E}-03$ \\
\hline$T_{\theta}+6$ & N/A & N/A & $N / A$ & N/A & N/A & $\mathrm{N} / \mathrm{A}$ & $\mathrm{N} / \mathrm{A}$ & N/A \\
\hline$T h+4$ & N/A & N/A & N/A & N/A & N/A & $N / A$ & N/A & $N / A$ \\
\hline $\mathrm{TI}+4$ & $\mathrm{~N} / \mathrm{A}$ & N/A & N/A & N/A & $N / A$ & N/A & N/A & N/A \\
\hline TOC & & & & & & & & $1.98 \mathrm{E}+01$ \\
\hline $\mathrm{UO} 2+2$ & $6.63 E+02$ & $4.00 E+02$ & $1.41 \mathrm{E}+00$ & $8.80 E+02$ & $7.85 E+01$ & $5.93 E+02$ & $3.02 \mathrm{E}+\mathrm{O} 2$ & $2.54 \mathrm{E}+02$ \\
\hline $2 n+2$ & N/A & N/A & N/A & N/A & $\mathrm{N} / \mathrm{A}$ & N/A & N/A & $N / A$ \\
\hline $\mathrm{ZrO} 2: 2 \mathrm{H} 2 \mathrm{O}$ & & & & & & & & $1.81 \mathrm{E}-01$ \\
\hline Total & $2.07 E+06$ & $1.39 E+06$ & $2.99 E+04$ & $5.65 E+06$ & $2.79 E+05$ & $7.86 E+05$ & $3.79 E+05$ & $3.72 \mathrm{E}+05$ \\
\hline
\end{tabular}


Table A-1. Chemical Inventory Estimates for Single-Shell Tanks

\begin{tabular}{|c|c|c|c|c|c|c|c|c|}
\hline $\begin{array}{c}\text { Soluble } \\
(\mathbf{k g})\end{array}$ & $T-104$ & $T-105$ & $T-106$ & $T-107$ & $\mathrm{~T}-108$ & $T-109$ & $\mathrm{~T}-110$ & T-1 11 \\
\hline $\mathrm{Ag}+$ & N/A & N/A & N/A & N/A & $N / A$ & $N / A$ & N/A & $\mathrm{N} / \mathrm{A}$ \\
\hline Al $(\mathrm{OH}) 4$ & $1.52 E+04$ & $1.89 E+01$ & $2.11 E+03$ & $5.52 E+03$ & $8.69 \mathrm{E}-02$ & & & \\
\hline$A s+5$ & N/A & N/A & N/A & N/A & N/A & $\mathrm{N} / \mathrm{A}$ & N/A & N/A \\
\hline$B+3$ & N/A & N/A & N/A & $N / A$ & $N / A$ & $N / A$ & N/A & $\mathrm{N} / \mathrm{A}$ \\
\hline $\mathrm{Ba}+2$ & $\mathrm{~N} / \mathrm{A}$ & N/A & N/A & $\mathrm{N} / \mathrm{A}$ & $\mathrm{N} / \mathrm{A}$ & N/A & $\mathrm{N} / \mathrm{A}$ & N/A \\
\hline$B \theta+2$ & $\mathrm{~N} / \mathrm{A}$ & N/A & N/A & N/A & $\mathrm{N} / \mathrm{A}$ & $N / A$ & N/A & N/A \\
\hline $\mathrm{Bi}+3$ & $1.01 E+02$ & $3.25 E+01$ & $1.61 \mathrm{E}+00$ & $3.78 \mathrm{E}+01$ & $5.06 \mathrm{E}=01$ & $1.13 E+02$ & $1.51 \mathrm{E}+02$ & $2.12 \mathrm{E}+02$ \\
\hline $\mathrm{Ca}+2$ & $1.34 E+00$ & 5.01E-01 & $8.60 \mathrm{E}-02$ & $4.64 \mathrm{E}-01$ & $9.75 \mathrm{E}-00$ & $2.45 E+01$ & $2.42 \mathrm{E}+00$ & $4.27 E+00$ \\
\hline $\mathrm{Cd}+2$ & $N / A$ & N/A & N/A & N/A & N/A & N/A & N/A & $N / A$ \\
\hline $\mathrm{Ce}+3$ & $1.17 \mathrm{E}+02$ & & 4.51E-01 & & 1.24E.01 & $6.83 E+00$ & & \\
\hline $\mathrm{Cr}-$ & $8.63 E+02$ & $3.11 \mathrm{E}+02$ & $3.59 E+01$ & $2.33 E+02$ & $2.57 E-02$ & $5.56 \mathrm{E}+02$ & $1.34 E+03$ & $1.62 \mathrm{E}+03$ \\
\hline $\mathrm{Co}+3$ & $N / A$ & N/A & N/A & N/A & $\mathrm{N} / \mathrm{A}$ & $N / A$ & N/A & N/A \\
\hline $\cos -2$ & $2.06 E+03$ & $7.73 E+02$ & $1.33 E+02$ & $7.16 E+02$ & $8.61 \mathrm{E}-02$ & $1.89 \mathrm{E}+03$ & $3.74 E+03$ & $6.58 E+03$ \\
\hline $\mathrm{Cr}(\mathrm{OH}) 4$ & $9.62 E+01$ & $2.03 E+01$ & $4.14 E+00$ & $2.57 E+01$ & $4.91 E-01$ & $1.14 E+02$ & $7.75 \mathrm{E}+01$ & $1.43 E+02$ \\
\hline $\mathrm{Cs}+$ & $1.92 \mathrm{E}-01$ & & $1.08 \mathrm{E}-02$ & & $2.24 E \cdot 02$ & $9.42 \mathrm{E}-02$ & & \\
\hline $\mathrm{Cu}+2$ & N/A & N/A & N/A & $\mathrm{N} / \mathrm{A}$ & $\mathrm{N} / \mathrm{A}$ & N/A & N/A & N/A \\
\hline $\mathrm{F}-$ & $1.64 \mathrm{E}+04$ & $4.50 E+03$ & $3.22 E+02$ & $5.46 \mathrm{E}+03$ & $2.94 \mathrm{E}-03$ & $5.23 E+03$ & $2.10 \mathrm{E}+04$ & $2.24 E+04$ \\
\hline $\mathrm{Fe}+3$ & $8.90 E+01$ & $3.75 E+01$ & $4.46 \mathrm{E}+00$ & $3.36 \mathrm{E}+01$ & $3.18 \mathrm{E}+01$ & $6.68 \mathrm{E}+01$ & $1.84 E+02$ & $3.17 \mathrm{E}+02$ \\
\hline $\mathrm{H} 2 \mathrm{O}$ & $2.80 E+06$ & $6.22 \mathrm{E}+05$ & $2.21 E+05$ & $9.55 E+05$ & $4.72 E--05$ & $8.12 E+05$ & $2.61 \mathrm{E}+06$ & $1.98 \mathrm{E}+06$ \\
\hline $\mathrm{Hg}+2$ & N/A & N/A & N/A & $\mathrm{N} / \mathrm{A}$ & $N / A$ & $N / A$ & $N / A$ & N/A \\
\hline $\mathrm{K}+$ & & & & & & & $8.56 \mathrm{E}+01$ & $3.07 E+02$ \\
\hline $\mathrm{La}+3$ & & & & & & & $5.84 \mathrm{E}-01$ & $2.10 E+00$ \\
\hline $\mathrm{Mg}+2$ & $N / A$ & N/A & N/A & $\mathrm{N} / \mathrm{A}$ & $\mathrm{N} / \mathrm{A}$ & N/A & $N / A$ & N/A \\
\hline $\mathrm{Mn}+4$ & & & & & & & $1.14 \mathrm{E}-02$ & $4.10 \mathrm{E}-02$ \\
\hline $\mathrm{Mo}+6$ & $N / A$ & N/A & N/A & $\mathrm{N} / \mathrm{A}$ & $N / A$ & N/A & $N / A$ & N/A \\
\hline $\mathrm{Na}+$ & $3.23 E+05$ & $7.27 E+04$ & $1.05 E+04$ & $1.09 E+05$ & $5.65 E-04$ & $9.93 \mathrm{E}+04$ & 3.07E + 05 & $2.40 E+05$ \\
\hline $\mathrm{Ni}+3$ & $1.65 \mathrm{E}+00$ & $7.06 E-01$ & $1.20 \mathrm{E}-01$ & 6.04E-01 & $6.06 \mathrm{E}+00$ & $1.51 \mathrm{E}+01$ & $3.55 E+00$ & $6.59 E+00$ \\
\hline NO2- & $1.15 E+04$ & $7.52 \mathrm{E}+02$ & $2.02 E+03$ & $3.08 \mathrm{E}+03$ & $1.36 \mathrm{E}+-03$ & $2.20 \mathrm{E}+03$ & & \\
\hline NO3- & $1.09 E+05$ & $4.24 E+04$ & $6.49 E+03$ & $2.84 E+04$ & $4.16 E=04$ & $9.42 \mathrm{E}+04$ & $1.99 \mathrm{E}+05$ & $2.36 \mathrm{E}+05$ \\
\hline $\mathrm{OH}-$ & $1.48 \mathrm{E}+05$ & $2.93 E+04$ & $3.88 E+03$ & $5.24 E+04$ & $1.70 E=04$ & $2.25 \mathrm{E}+04$ & $1.21 \mathrm{E}+05$ & $6.78 \mathrm{E}+04$ \\
\hline \multicolumn{9}{|l|}{$\mathrm{Pb}+4$} \\
\hline $\mathrm{PO} 4-3$ & $9.01 E+04$ & $1.85 E+04$ & $1.59 E+03$ & $3.21 E+04$ & $1.98 E--04$ & $3.71 E+04$ & $7.07 \mathrm{E}+04$ & $4.44 E+04$ \\
\hline $\mathbf{R b}+$ & N/A & N/A & N/A & $N / A$ & $\mathrm{~N} / \mathrm{A}$ & N/A & $N / A$ & $\mathrm{~N} / \mathrm{A}$ \\
\hline $\mathrm{Re}+7$ & $N / A$ & N/A & N/A & $N / A$ & $N / A$ & N/A & N/A & $\mathrm{N} / \mathrm{A}$ \\
\hline$R h+3$ & $N / A$ & N/A & N/A & $\mathrm{N} / \mathrm{A}$ & $N / A$ & N/A & N/A & N/A \\
\hline $\mathbf{R u}+3$ & $N / A$ & N/A & N/A & $\mathrm{N} / \mathrm{A}$ & $N / A$ & N/A & N/A & N/A \\
\hline $\mathrm{Se}+6$ & N/A & N/A & $N / A$ & $N / A$ & $N / A$ & $N / A$ & N/A & N/A \\
\hline $5 i+4$ & & & & & & $1.11 E+02$ & & \\
\hline 504-2 & $2.86 \mathrm{E}+03$ & $5.63 E+02$ & $7.57 E+01$ & $7.62 E+02$ & $2.16 E-03$ & $5.16 \mathrm{E}+03$ & $1.91 \mathrm{E}+03$ & $2.07 \mathrm{E}+03$ \\
\hline$S_{r}+2$ & $2.03 E-03$ & 2.47E-04 & $7.71 \mathrm{E}-02$ & $1.79 \mathrm{E}-03$ & 1.79E.04 & & $1.11 \mathrm{E}+01$ & $3.99 E+01$ \\
\hline Te +6 & N/A & N/A & $N / A$ & $\mathrm{~N} / \mathrm{A}$ & $\mathrm{N} / \mathrm{A}$ & N/A & $N / A$ & $\mathrm{~N} / \mathrm{A}$ \\
\hline $\mathrm{Th}+4$ & $N / A$ & N/A & N/A & $\mathrm{N} / \mathrm{A}$ & N/A & $N / A$ & $N / A$ & N/A \\
\hline $\mathrm{TI}+4$ & $N / A$ & N/A & N/A & N/A & $N / A$ & N/A & N/A & N/A \\
\hline TOC & & & & & & & $2.55 \mathrm{E}+02$ & $8.87 E+02$ \\
\hline UO2 +2 & $2.42 \mathrm{E}+01$ & $2.54 \mathrm{E}+00$ & $9.85 E+01$ & $3.07 \varepsilon+00$ & $2.64 \mathrm{E}-00$ & $5.43 E+00$ & $1.17 E+00$ & $1.24 E+00$ \\
\hline$Z n+2$ & $\mathrm{~N} / \mathrm{A}$ & N/A & N/A & $\mathrm{N} / \mathrm{A}$ & N/A & N/A & $N / A$ & N/A \\
\hline $\mathrm{ZrO} 2: 2 \mathrm{H} 2 \mathrm{O}$ & $1.86 \mathrm{E}+02$ & $8.80 \mathrm{E}+00$ & $3.39 E+00$ & $6.51 \mathrm{E}+01$ & $1.04 \mathrm{E}-01$ & & & \\
\hline Total & $3.52 \mathrm{E}+06$ & $7.92 E+05$ & $2.48 \mathrm{E}+05$ & $1.19 E+06$ & $6.16 \mathrm{E}=05$ & $1.08 E+06$ & $3.34 \mathrm{E}+06$ & $2.61 E+O B$ \\
\hline
\end{tabular}


Table A-1. Chemical inventory Estimates for Single-Shell Tanks

\begin{tabular}{|c|c|c|c|c|c|c|c|c|}
\hline $\begin{array}{c}\text { Soluble } \\
(\mathrm{kg})\end{array}$ & $T-112$ & $T-201$ & $\mathrm{~T} \cdot 202$ & $T-203$ & $T-204$ & TX-101 & $T X-102$ & TX-103 \\
\hline $\mathrm{Ag}+$ & N/A & N/A & N/A & $N / A$ & $\mathrm{~N} / \mathrm{A}$ & $N / A$ & N/A & N/A \\
\hline AllOH)4 & & & & & & $1.18 E+04$ & $7.14 E+03$ & $9.95 E+03$ \\
\hline$A B+5$ & N/A & N/A & N/A & $\mathrm{N} / \mathrm{A}$ & $\mathrm{N} / \mathrm{A}$ & $N / A$ & N/A & N/A \\
\hline$B+3$ & N/A & N/A & N/A & N/A & $N / A$ & N/A & N/A & N/A \\
\hline $\mathrm{Ba}+2$ & $N / A$ & N/A & $\mathrm{N} / \mathrm{A}$ & N/A & $N / A$ & N/A & N/A & N/A \\
\hline $\mathrm{Be}+2$ & N/A & N/A & $\mathrm{N} / \mathrm{A}$ & N/A & N/A & $N / A$ & N/A & N/A \\
\hline $\mathrm{Bi}+3$ & $3.06 \mathrm{E}+01$ & $1.06 \mathrm{E}+01$ & $7.99 E+00$ & $1.33 E+01$ & $1.44 \mathrm{E}-01$ & $9.25 \mathrm{E}-01$ & $1.28 \mathrm{E}+01$ & $1.79 E+01$ \\
\hline $\mathrm{Ca}+2$ & $6.56 \mathrm{E}-01$ & $2.73 \mathrm{E}-01$ & $2.05 \mathrm{E}-01$ & $3.41 \mathrm{E}-01$ & 3.70E 01 & $2.82 E+00$ & $3.39 E+01$ & $4.72 \mathrm{E}+01$ \\
\hline$C d+2$ & $\mathrm{~N} / \mathrm{A}$ & N/A & $\mathrm{N} / \mathrm{A}$ & $\mathrm{N} / \mathrm{A}$ & N/A & N/A & N/A & N/A \\
\hline $\mathrm{Ce}+3$ & & & & & & & $4.29 \mathrm{E}-02$ & 4.15E-01 \\
\hline ci. & $2.10 E+02$ & $1.12 \mathrm{E}+02$ & $8.45 E+01$ & $1.40 E+02$ & $1.52 \mathrm{E}-\mathrm{-O2}$ & $5.50 E+02$ & $1.52 \mathrm{E}+\mathrm{O} 3$ & $2.12 \mathrm{E}+03$ \\
\hline $\mathrm{Co}+3$ & $\mathrm{~N} / \mathrm{A}$ & N/A & N/A & N/A & $N / A$ & $\mathrm{~N} / \mathrm{A}$ & N/A & $\mathrm{N} / \mathrm{A}$ \\
\hline $\cos -2$ & $1.01 E+03$ & $4.21 E+02$ & $3.17 \mathrm{E}+02$ & $5.26 \mathrm{E}+02$ & $5.71 \mathrm{E}-\mathrm{O} 2$ & $1.11 E+03$ & $2.87 E+03$ & $4.02 E+03$ \\
\hline $\mathrm{Cr}(\mathrm{OH}) 4$ & $2.31 E+01$ & $6.18 \mathrm{E}+00$ & $4.64 \mathrm{E}+00$ & $7.71 E+00$ & $8.37 \mathrm{E}-00$ & $1.09 E+03$ & $2.15 E+03$ & $3.00 E+03$ \\
\hline $\mathrm{Cs}+$ & & & & & & & 3.77E-02 & $5.24 \mathrm{E}-02$ \\
\hline $\mathrm{Cu}+2$ & N/A & N/A & N/A & N/A & N/A & $N / A$ & N/A & $N / A$ \\
\hline F- & $8.74 E+02$ & $8.71 E+03$ & $6.55 \mathrm{E}+03$ & $1.09 E+04$ & $1.18 \mathrm{E}+04$ & $4.29 \mathrm{E}+01$ & $5.96 \mathrm{E}+02$ & $8.30 E+02$ \\
\hline $\mathrm{Fe}+3$ & $4.98 E+01$ & $1.40 E+01$ & $1.06 \mathrm{E}+01$ & $1.75 E+01$ & $1.90 \mathrm{E}+01$ & $2.64 E+01$ & $9.28 E+01$ & $1.29 E+02$ \\
\hline $\mathrm{H} 2 \mathrm{O}$ & $1.61 \mathrm{E}+05$ & $1.77 E+05$ & $1.33 \mathrm{E}+05$ & $2.21 \mathrm{E}+05$ & $2.40 E-05$ & $9.30 E+0.5$ & $1.84 \mathrm{E}+06$ & $2.55 E+06$ \\
\hline $\mathrm{Hg}+2$ & N/A & N/A & $\mathrm{N} / \mathrm{A}$ & N/A & N/A & N/A & $N / A$ & $\mathrm{~N} / \mathrm{A}$ \\
\hline $\mathrm{K}+$ & & $2.39 E+O 2$ & $1.80 E+02$ & $2.99 E+02$ & $3.24 E+02$ & & & \\
\hline $\mathrm{La}+3$ & & $1.63 E+00$ & $1.23 E+00$ & $2.04 E+00$ & $2.21 \mathrm{E}+00$ & & & \\
\hline $\mathrm{Mg}+2$ & N/A & N/A & $N / A$ & N/A & $N / A$ & N/A & N/A & N/A \\
\hline $\mathrm{Mn}+4$ & & $3.19 \mathrm{E}-02$ & $2.40 \mathrm{E}-\mathrm{O} 2$ & $3.98 \mathrm{E}-02$ & 4.32E 02 & & & \\
\hline $\mathrm{Mo}+6$ & N/A & $\mathrm{N} / \mathrm{A}$ & N/A & $\mathrm{N} / \mathrm{A}$ & $N / A$ & N/A & N/A & N/A \\
\hline $\mathrm{Na}+$ & $2.02 E+04$ & $2.15 E+04$ & $1.62 E+04$ & $2.69 \mathrm{E}+04$ & $2.92 \mathrm{E}+0.4$ & $4.82 \mathrm{E}+04$ & $2.54 \mathrm{E}+05$ & $3.54 \mathrm{E}+05$ \\
\hline $\mathrm{Ni}+3$ & $1.03 E+00$ & $4.21 \mathrm{E}-01$ & $3.16 \mathrm{E}-01$ & $5.25 \mathrm{E}-01$ & $5.70 \mathrm{OE} 01$ & $2.02 \mathrm{E}+00$ & $2.09 E+01$ & $2.91 E+01$ \\
\hline NO2- & & & & & & & $2.41 E+04$ & $3.35 E+04$ \\
\hline NO3- & $2.89 E+04$ & $2.12 E+04$ & $1.60 E+04$ & $2.65 E+04$ & $2.88 \mathrm{E}+04$ & $1.15 E+05$ & $6.09 E+05$ & $8.72 E+05$ \\
\hline OH- & $3.87 E+03$ & $1.50 E+03$ & $1.13 E+03$ & $1.88 E+03$ & $2.04 \mathrm{E}+\mathrm{O} 3$ & & $6.47 E+03$ & $1.89 E+03$ \\
\hline \multicolumn{9}{|l|}{$\mathrm{Pb}+4$} \\
\hline PO4-3 & $3.32 E+03$ & $7.72 E+02$ & $5.80 \mathrm{E}+02$ & $9.63 E+02$ & $1.05 E+03$ & $3.68 \mathrm{E}+02$ & $1.85 E+03$ & $2.60 E+03$ \\
\hline $\mathbf{R b}+$ & $\mathrm{N} / \mathrm{A}$ & N/A & N/A & $\mathrm{N} / \mathrm{A}$ & $N / A$ & N/A & $N / A$ & N/A \\
\hline $\mathrm{Re}+7$ & N/A & N/A & N/A & N/A & $N / A$ & N/A & $N / A$ & $N / A$ \\
\hline $\mathrm{Rh}+3$ & N/A & $\mathrm{N} / \mathrm{A}$ & N/A & N/A & $N / A$ & N/A & N/A & $\mathrm{N} / \mathrm{A}$ \\
\hline $\mathrm{Ru}+\mathbf{3}$ & $N / A$ & N/A & N/A & N/A & $N / A$ & N/A & N/A & N/A \\
\hline$S_{\theta}+6$ & N/A & N/A & N/A & N/A & $N / A$ & N/A & N/A & $N / A$ \\
\hline \multicolumn{9}{|l|}{$5 i+4$} \\
\hline so4-2 & $2.82 E+02$ & $1.29 E+01$ & $9.69 E+00$ & $1.61 E+01$ & $1.75 E+01$ & $8.10 E+02$ & $4.22 E+03$ & $5.90 E+03$ \\
\hline$S r+2$ & $2.32 \mathrm{E}-04$ & $3.11 E+01$ & $2.34 \mathrm{E}+01$ & $3.88 \mathrm{E}+01$ & $4.21 \mathrm{E}+01$ & $4.33 \mathrm{E}-04$ & $1.66 \mathrm{E}-05$ & $3.69 \mathrm{E}-05$ \\
\hline$T e+6$ & N/A & N/A & N/A & N/A & $N / A$ & N/A & $N / A$ & N/A \\
\hline$T h+4$ & N/A & $N / A$ & $\mathrm{~N} / \mathrm{A}$ & N/A & $N / A_{1}$ & N/A & $\mathrm{N} / \mathrm{A}$ & N/A \\
\hline $\mathrm{TI}+4$ & N/A & N/A & N/A & $\mathrm{N} / \mathrm{A}$ & $\mathrm{N} / \mathrm{A}_{1}$ & N/A & $\mathrm{N} / \mathrm{A}$ & N/A \\
\hline TOC & & $7.64 \mathrm{E}+02$ & $5.75 E+02$ & $9.54 \mathrm{E}+02$ & $1.04 \mathrm{E}+03$ & $3.21 \mathrm{E}+01$ & $5.28 E+02$ & $7.36 \mathrm{E}+02$ \\
\hline $\mathrm{UO} 2+2$ & $1.68 \mathrm{E}-01$ & & & & & $2.27 E+02$ & $2.54 E+02$ & $3.66 \mathrm{E}+02$ \\
\hline $\mathrm{Zn}+2$ & N/A & N/A & N/A & N/A & $\mathbf{N} / \boldsymbol{A}_{\mathbf{1}}$ & N/A & $\mathrm{N} / \mathrm{A}$ & N/A \\
\hline \multicolumn{9}{|l|}{$\mathrm{ZrO} 2: 2 \mathrm{H} 2 \mathrm{O}$} \\
\hline Total & $2.20 E+05$ & $2.32 E+05$ & $1.75 E+05$ & $2.90 E+05$ & $3.15 E+05$ & $1.11 E+06$ & $2.76 E+06$ & $3.84 E+06$ \\
\hline
\end{tabular}


Table A-1. Chemical Inventory Estimates for Single-Shell Tanks

\begin{tabular}{|c|c|c|c|c|c|c|c|c|}
\hline $\begin{array}{c}\text { Soluble } \\
(\mathrm{kg})\end{array}$ & $T X-104$ & $T X-105$ & $T X-106$ & TX-107 & $T X-108$ & TX-109 & TX-110 & TX-111 \\
\hline $\mathrm{Ag}+$ & N/A & N/A & N/A & $N / A$ & $N / A$ & $N / A$ & N/A & N/A \\
\hline Al(OH)4 & $2.96 \mathrm{E}+03$ & $3.87 E+04$ & $2.95 E+04$ & $1.74 \mathrm{E}+03$ & $8.24 E-03$ & $1.62 \mathrm{E}+04$ & $2.84 E+04$ & $2.21 E+04$ \\
\hline$A s+5$ & N/A & N/A & N/A & N/A & N/A & N/A & N/A & N/A \\
\hline$B+3$ & N/A & N/A & N/A & N/A & $\mathrm{N} / \mathrm{A}$ & N/A & N/A & $N / A$ \\
\hline $\mathrm{Ba}+2$ & N/A & N/A & N/A & N/A & $\mathrm{N} / \mathrm{A}$ & $N / A$ & N/A & N/A \\
\hline $\mathrm{Be}+2$ & N/A & N/A & $N / A$ & $\mathrm{~N} / \mathrm{A}$ & N/A & N/A & N/A & N/A \\
\hline $\mathrm{Bi}+3$ & $5.32 \mathrm{E}+00$ & $6.96 \mathrm{E}+01$ & $5.18 E+01$ & $3.12 \mathrm{E}+00$ & $1.48 \mathrm{E}=01$ & $1.11 \mathrm{E}+02$ & $5.63 \mathrm{E}+01$ & $4.47 E+01$ \\
\hline $\mathrm{Ca}+2$ & $1.41 E+01$ & $1.84 E+02$ & $1.37 E+02$ & $8.28 E+00$ & $3.34 \mathrm{E}-01$ & $1.36 \mathrm{E}+00$ & $1.30 \mathrm{E}+02$ & $9.99 E+01$ \\
\hline$C d+2$ & N/A & N/A & N/A & N/A & $N / A$ & N/A & N/A & N/A \\
\hline $\mathrm{Ca}+3$ & & $5.03 E-01$ & & & $2.55 \mathrm{E}-02$ & $2.20 \mathrm{E}+02$ & $1.71 E+02$ & $4.45 E+01$ \\
\hline Cl- & 6.37E + 02 & $8.25 E+03$ & $6.15 E+03$ & $3.72 \varepsilon+02$ & $1.79 \mathrm{E}-03$ & $6.86 \mathrm{E}+02$ & $5.90 \mathrm{E}+03$ & $4.57 E+03$ \\
\hline $\mathrm{Co}+3$ & $N / A$ & $N / A$ & N/A & N/A & $N / A$ & N/A & N/A & N/A \\
\hline $\mathrm{CO} 3-2$ & $2.87 \mathrm{E}+03$ & $1.56 \mathrm{E}+04$ & $1.09 E+04$ & $1.69 \varepsilon+03$ & $3.35 \mathrm{E}-03$ & $2.10 \mathrm{E}+03$ & $1.04 E+04$ & $8.04 E+03$ \\
\hline $\mathrm{Cr}(\mathrm{OH}) 4$ & 8.93E + 02 & $1.17 \mathrm{E}+04$ & $8.78 E+03$ & $5.24 E+02$ & $2.47 \mathrm{E}-03$ & $7.54 \mathrm{E}+01$ & $8.25 E+03$ & $6.34 E+03$ \\
\hline $\mathrm{Cs}+$ & & $3.66 E-01$ & 5.92E-01 & & $2.19 E-00$ & $1.57 E+01$ & $6.61 \mathrm{E}+00$ & $1.50 \mathrm{E}+00$ \\
\hline $\mathrm{Cu}+2$ & N/A & N/A & $\mathrm{N} / \mathrm{A}$ & $\mathrm{N} / \mathrm{A}$ & $N / A$ & $N / A$ & N/A & $\mathrm{N} / \mathrm{A}$ \\
\hline F. & $2.47 E+02$ & $3.23 E+03$ & $2.40 E+03$ & $1.45 E+02$ & $6.88 \mathrm{E}-02$ & $1.60 \mathrm{E}+04$ & $3.56 \mathrm{E}+03$ & $3.14 E+03$ \\
\hline $\mathrm{F}_{\theta}+3$ & $4.13 E+01$ & $5.03 E+02$ & $3.99 E+02$ & $2.38 E+01$ & $3.30 \mathrm{E}-01$ & $9.86 \mathrm{E}+01$ & $3.61 E+02$ & $2.78 \mathrm{E}+02$ \\
\hline $\mathrm{H} 2 \mathrm{O}$ & $8.76 E+05$ & $9.91 E+06$ & $7.34 E+06$ & $5.02 E+05$ & $2.13 E-06$ & $2.80 E+06$ & $7.18 E+06$ & $5.57 E+06$ \\
\hline $\mathrm{Hg}+2$ & N/A & N/A & N/A & N/A & N/A & $N / A$ & N/A & $\mathrm{N} / \mathrm{A}$ \\
\hline \multicolumn{9}{|l|}{$\mathrm{K}+$} \\
\hline \multicolumn{9}{|l|}{$\mathrm{La}+3$} \\
\hline $\mathrm{Mg}+2$ & $N / A$ & N/A & N/A & N/A & N/A & $\mathrm{N} / \mathrm{A}$ & N/A & $\mathrm{N} / \mathrm{A}$ \\
\hline \multicolumn{9}{|l|}{$\mathrm{Mn}+4$} \\
\hline $\mathrm{Mo}+6$ & N/A & N/A & $N / A$ & $\mathrm{~N} / \mathrm{A}$ & $N / A$ & $N / A$ & N/A & $N / A$ \\
\hline $\mathrm{Na}+$ & $1.19 E+05$ & $1.37 E+06$ & $1.02 E+06$ & $6.83 \mathrm{E}+04$ & $2.95 E+05$ & $3.21 \mathrm{E}+05$ & $9.92 \mathrm{E}+05$ & $7.69 \varepsilon+05$ \\
\hline $\mathrm{Ni}+3$ & 8.76E +00 & $1.13 E+02$ & $8.47 E+01$ & $5.13 E+00$ & $1.56 \mathrm{E}-01$ & $1.77 \mathrm{E}+00$ & $8.03 E+01$ & $6.17 E+01$ \\
\hline NO2- & $9.98 \mathrm{E}+03$ & $1.31 E+05$ & $9.70 E+04$ & $5.86 \mathrm{E}+03$ & $2.78 \mathrm{E}-04$ & $9.03 E+03$ & $9.33 E+04$ & $7.21 E+04$ \\
\hline NO3- & $2.61 E+05$ & $3.39 E+06$ & $2.53 E+06$ & $1.53 E+05$ & $7.27 \mathrm{E}+05$ & $8.36 \mathrm{E}+04$ & $2.41 \mathrm{E}+06$ & $1.86 \varepsilon+06$ \\
\hline $\mathrm{OH}$ & $8.52 E+03$ & $6.07 E+03$ & $1.58 \mathrm{E}+03$ & $4.23 E+03$ & $1.17 \mathrm{E}+03$ & $1.54 \mathrm{E}+0.5$ & 1.24E + 04 & $1.23 E+04$ \\
\hline \multicolumn{9}{|l|}{$\mathrm{Pb}+4$} \\
\hline PO4-3 & $2.18 \mathrm{E}+03$ & $9.80 \mathrm{E}+03$ & $6.91 E+03$ & $1.07 E+03$ & $1.66 \mathrm{E}+03$ & $9.42 \mathrm{E}+04$ & $1.3 t E+04$ & $1.18 E+04$ \\
\hline $\mathrm{Rb}+$ & N/A & N/A & $N / A$ & N/A & $N / A$ & N/A & N/A & $\mathrm{N} / \mathrm{A}$ \\
\hline$R e+7$ & N/A & N/A & $\mathrm{N} / \mathrm{A}$ & N/A & $N / A$ & N/A & N/A & $N / A$ \\
\hline $\mathrm{Rh}+3$ & N/A & N/A & N/A & $N / A$ & $N / A$ & N/A & $\mathrm{N} / \mathrm{A}$ & N/A \\
\hline$R u+3$ & $N / A$ & N/A & N/A & $\mathrm{N} / \mathrm{A}$ & $N / A$ & N/A & N/A & N/A \\
\hline $\mathrm{Se}+6$ & $\mathrm{~N} / \mathrm{A}$ & $\mathrm{N} / \mathrm{A}$ & N/A & $N / A$ & $N / A$ & N/A & N/A & $N / A$ \\
\hline \multicolumn{9}{|l|}{$\mathrm{Si}+4$} \\
\hline SO4-2 & $3.86 \mathrm{E}+03$ & $2.25 E+04$ & $1.61 \varepsilon+04$ & $1.95 E+03$ & $5.34 \mathrm{E}+03$ & $2.24 \mathrm{E}+03$ & $1.55 \mathrm{E}+04$ & $1.20 \mathrm{E}+04$ \\
\hline$s r+2$ & & $1.62 \mathrm{E}-04$ & & & $2.61 \mathrm{E} 04$ & $7.48 \mathrm{E}-03$ & 5.24E-03 & $2.47 E-03$ \\
\hline$T_{\theta}+6$ & N/A & N/A & N/A & N/A & $N / A$. & N/A & N/A & N/A \\
\hline Th+4 & N/A & N/A & $\mathrm{N} / \mathrm{A}$ & N/A & $\mathrm{N} / \mathrm{A}$ & N/A & $\mathrm{N} / \mathrm{A}$ & $\mathrm{N} / \mathrm{A}$ \\
\hline$T I+4$ & N/A & N/A & $N / A$ & N/A & $N / A$ & $N / A$ & $N / A$ & N/A \\
\hline TOC & $2.13 E+02$ & $2.87 E+03$ & $2.13 E+03$ & $1.26 \mathrm{E}+02$ & $6.07 E+02$ & & $1.99 E+03$ & $1.51 E+03$ \\
\hline $\mathrm{UO} 2+2$ & $1.06 \mathrm{E}+03$ & $1.53 E+03$ & $6.28 \mathrm{E}+02$ & $7.92 \mathrm{E}+02$ & $3.38 \mathrm{E}+02$ & $9.02 E+00$ & $5.48 \mathrm{E}+02$ & $4.23 E+02$ \\
\hline$Z n+2$ & N/A & N/A & $\mathrm{N} / \mathrm{A}$ & N/A & $\mathrm{N} / \mathrm{A}$ & N/A & N/A & $\mathrm{N} / \mathrm{A}$ \\
\hline $\mathrm{ZrO} 2: 2 \mathrm{H} 2 \mathrm{O}$ & & & & & & $1.91 \mathrm{E}+02$ & $1.40 \mathrm{E}+01$ & $1.45 \mathrm{E}+01$ \\
\hline Total & $1.29 E+06$ & $1.49 E+07$ & $1.11 \mathrm{E}+07$ & $7.41 E+05$ & $3.20 \mathrm{E}+06$ & $3.50 E+06$ & $1.08 \mathrm{E}+07$ & $8.35 E+06$ \\
\hline
\end{tabular}


Table A-1. Chemical Inventory Estimates for Single-Shell Tanks

\begin{tabular}{|c|c|c|c|c|c|c|c|c|}
\hline $\begin{array}{c}\text { Soluble } \\
(\mathrm{kg})\end{array}$ & $T X-112$ & $T X-113$ & TX-114 & $T X-115$ & $T X-116$ & TX-117 & $T X-118$ & TY-101 \\
\hline $\mathrm{Ag}_{\mathrm{g}+}$ & $N / A$ & N/A & N/A & N/A & $N / A$ & $N / A$ & N/A & N/A \\
\hline Al(OH)4- & $4.02 E+04$ & $3.46 \mathrm{E}+04$ & $3.05 E+04$ & $4.06 E+04$ & $1.77 E+04$ & $2.66 \mathrm{E}+04$ & $2.36 \mathrm{E}+04$ & \\
\hline$A s+5$ & N/A & N/A & N/A & N/A & $N / A$ & N/A & N/A & N/A \\
\hline $\mathrm{B}+3$ & N/A & N/A & N/A & N/A & N/A & N/A & $\mathrm{N} / \mathrm{A}$ & N/A \\
\hline $\mathrm{Ba}+2$ & N/A & N/A & $\mathrm{N} / \mathrm{A}$ & $\mathrm{N} / \mathrm{A}$ & $N / A$ & N/A & $\mathrm{N} / \mathrm{A}$ & N/A \\
\hline $\mathrm{Be}+2$ & N/A & N/A & N/A & N/A & N/A & N/A & $\mathrm{N} / \mathrm{A}$ & N/A \\
\hline $\mathrm{Bi}+3$ & $1.19 E+02$ & $1.02 E+02$ & $1.69 \mathrm{E}+02$ & $7.31 E+01$ & $6.60 \mathrm{E}+\mathrm{O} 22$ & $4.31 \mathrm{E}+02$ & $4.73 \mathrm{E}+01$ & $1.33 E+02$ \\
\hline $\mathrm{Ca}+2$ & $2.01 E+02$ & $1.30 E+02$ & $1.69 \mathrm{E}+02$ & $1.93 E+02$ & $2.24 \mathrm{E}+02$ & $2.12 \mathrm{E}+02$ & $9.69 \mathrm{E}+01$ & $2.00 E+01$ \\
\hline $\mathrm{Cd}+2$ & N/A & N/A & N/A & N/A & N/A & N/A & N/A & N/A \\
\hline $\mathrm{Ce}+3$ & $2.25 E+02$ & $1.45 E+02$ & $4.04 \mathrm{E}+01$ & $6.25 \mathrm{E}+01$ & $3.37 E-01$ & $1.37 \mathrm{E}+01$ & $1.40 E+02$ & $5.67 E+00$ \\
\hline $\mathrm{Cl}-$ & $8.80 E+03$ & $6.13 E+03$ & $7.04 \mathrm{E}+03$ & $8.75 E+03$ & $7.03 E-03$ & $7.67 \mathrm{E}+03$ & $4.79 E+03$ & $4.99 E+02$ \\
\hline $\mathrm{Co}+3$ & N/A & N/A & N/A & N/A & N/A & N/A & N/A & N/A \\
\hline $\mathrm{CO} 3-2$ & $1.59 \mathrm{E}+04$ & $1.12 E+04$ & $1.33 E+04$ & $1.55 E+04$ & $1.74 \mathrm{E}+04$ & $1.66 \mathrm{E}+04$ & $8.58 E+03$ & $2.45 E+03$ \\
\hline $\mathrm{Cr}(\mathrm{OH}) 4-$ & $1.22 \mathrm{E}+04$ & $8.24 E+03$ & $9.29 E+03$ & $1.22 \mathrm{E}+04$ & $6.21 E+03$ & $8.56 \mathrm{E}+03$ & $5.99 \mathrm{E}+03$ & $9.14 \mathrm{E}+01$ \\
\hline $\mathrm{Cs}+$ & $1.16 E+00$ & $3.98 \mathrm{E}-01$ & $1.16 E+00$ & $6.48 E-01$ & $2.75 \mathrm{E} 01$ & $1.12 \mathrm{E}-01$ & $1.84 E+01$ & $1.07 \mathrm{E}-01$ \\
\hline $\mathrm{Cu}+2$ & N/A & N/A & N/A & N/A & N/A & N/A & $N / A$ & N/A \\
\hline F- & $5.53 E+03$ & $9.89 \mathrm{E}+03$ & $7.93 E+03$ & $3.39 E+03$ & $3.06 E+04$ & $2.00 \mathrm{E}+04$ & $2.20 E+03$ & $8.83 E+03$ \\
\hline $\mathrm{Fe}+3$ & $5.49 E+02$ & $4.00 E+02$ & $4.63 E+02$ & $5.36 E+02$ & $6.11 E+02$ & $5.78 \mathrm{E}+02$ & $2.72 \mathrm{E}+02$ & $7.18 \mathrm{E}+01$ \\
\hline $\mathrm{H} 2 \mathrm{O}$ & $1.06 \mathrm{E}+07$ & $8.26 \mathrm{E}+06$ & $8.58 \mathrm{E}+06$ & $1.04 \mathrm{E}+07$ & $9.20 \mathrm{E}+06$ & $9.65 E+06$ & $5.26 \mathrm{E}+06$ & $1.72 \mathrm{E}+06$ \\
\hline $\mathrm{Hg}+2$ & N/A & N/A & N/A & N/A & $\mathrm{N} / \mathrm{A}$ & N/A & $\mathrm{N} / \mathrm{A}$ & $N / A$ \\
\hline \multicolumn{9}{|l|}{$k+$} \\
\hline \multicolumn{9}{|l|}{$\mathrm{La}+3$} \\
\hline $\mathrm{Mg}+2$ & $N / A$ & N/A & N/A & N/A & N/A & N/A & $\mathrm{N} / \mathrm{A}$ & $\mathrm{N} / \mathrm{A}$ \\
\hline \multicolumn{9}{|l|}{$\mathrm{Mn}+4$} \\
\hline $\mathrm{Mo}+6$ & N/A & N/A & $\mathrm{N} / \mathrm{A}$ & $N / A$ & N/A & N/A & N/A & $\mathrm{N} / \mathrm{A}$ \\
\hline $\mathrm{Na}+$ & $1.46 \mathrm{E}+06$ & $1.11 \mathrm{E}+06$ & $1.18 E+06$ & $1.44 E+06$ & $1.20 \mathrm{E}+06$ & $1.29 \mathrm{E}+06$ & $7.29 \mathrm{E}+05$ & $2.00 E+05$ \\
\hline $\mathrm{Ni}+3$ & $1.24 \mathrm{E}+02$ & $8.06 E+01$ & $1.04 E+02$ & $1.19 E+02$ & $1.38 \mathrm{E}+02$ & $1.31 \mathrm{E}+02$ & $5.99 E+01$ & $1.43 E+01$ \\
\hline NO2- & $1.37 E+05$ & $9.62 \mathrm{E}+04$ & $1.05 E+05$ & $1.37 E+05$ & $7.47 \mathrm{E}+04$ & $9.90 E+04$ & $6.72 \mathrm{E}+04$ & $2.43 E+03$ \\
\hline NO3- & $3.57 E+06$ & $2.43 E+06$ & $2.76 E+06$ & $3.57 \mathrm{E}+06$ & $2.15 \mathrm{E}+06$ & $2.70 \mathrm{E}+\mathrm{O} 6$ & $1.79 E+06$ & $8.06 E+04$ \\
\hline $\mathrm{OH}-$ & $1.12 \mathrm{E}+04$ & $7.43 E+04$ & $2.51 \mathrm{E}+04$ & $3.43 E+03$ & $1.23 \mathrm{E}+05$ & $7.57 E+04$ & $2.49 E+03$ & $8.62 E+04$ \\
\hline \multicolumn{9}{|l|}{$\mathrm{Pb}+4$} \\
\hline PO4-3 & $2.49 E+04$ & $5.11 E+04$ & $4.53 E+04$ & $9.72 \mathrm{E}+0.3$ & $2.11 E+05$ & $1.32 \mathrm{E}+05$ & $8.52 \mathrm{E}+03$ & $6.84 E+04$ \\
\hline $\mathrm{Rb}+$ & $N / A$ & $\mathrm{~N} / \mathrm{A}$ & $\mathrm{N} / \mathrm{A}$ & N/A & $N / A$ & N/A & $\mathrm{N} / \mathrm{A}$ & $\mathrm{N} / \mathrm{A}$ \\
\hline $\mathrm{Re}+7$ & N/A & N/A & N/A & $N / A$ & N/A & $\mathrm{N} / \mathrm{A}$ & N/A & N/A \\
\hline $\mathrm{Rh}+\mathbf{3}$ & N/A & N/A & $N / A$ & N/A & $N / A$ & N/A & N/A & $\mathrm{N} / \mathrm{A}$ \\
\hline$R u+3$ & $N / A$ & N/A & N/A & N/A & $N / A$ & N/A & N/A & $\mathrm{N} / \mathrm{A}$ \\
\hline $5 e+6$ & N/A & N/A & N/A & N/A & $\mathbf{N} / \mathbf{A}$ & N/A & N/A & $\mathrm{N} / \mathrm{A}$ \\
\hline$S i+4$ & & & & & & & & $1.57 E+02$ \\
\hline so4-2 & $2.45 \mathrm{E}+04$ & $1.62 E+04$ & $2.21 E+04$ & $2.37 E+04$ & $3.90 E+04$ & $3.26 \mathrm{E}+04$ & $1.16 \mathrm{E}+04$ & $4.30 E+03$ \\
\hline $\mathrm{Sr}+2$ & $3.92 E-04$ & $4.20 \mathrm{E}-04$ & 3.93E-05 & $1.53 \mathrm{E}-03$ & $1.99 \mathrm{E} 03$ & $3.79 \mathrm{E}-03$ & $3.90 E-02$ & 8.70E-04 \\
\hline$T e+6$ & N/A & N/A & N/A & N/A & $N / A$ & N/A & $\mathrm{N} / \mathrm{A}$ & N/A \\
\hline$T h+4$ & $\mathrm{~N} / \mathrm{A}$ & N/A & $\mathrm{N} / \mathrm{A}$ & $\mathrm{N} / \mathrm{A}$ & $\mathrm{N} / \boldsymbol{A}_{1}$ & N/A & N/A & N/A \\
\hline$T I+4$ & N/A & N/A & $\mathrm{N} / \mathrm{A}$ & N/A & $\mathbf{N} / \mathbf{A}_{1}$ & N/A & N/A & $\mathrm{N} / \mathrm{A}$ \\
\hline TOC & $2.98 \mathrm{E}+03$ & $1.90 E+03$ & $2.23 E+03$ & $3.01 E+03$ & $1.31 \mathrm{E}+03$ & $1.96 \mathrm{E}+03$ & $1.44 E+03$ & $2.13 E+03$ \\
\hline $\mathrm{UO} 2+2$ & $8.04 \mathrm{E}+02$ & 5.47E + 02 & $6.12 \mathrm{E}+02$ & $8.71 E+02$ & $3.99 \mathrm{E}+\mathrm{O} 2$ & $5.59 \varepsilon+02$ & $3.96 E+02$ & $6.48 \mathrm{E}+00$ \\
\hline $2 n+2$ & $N / A$ & $\mathrm{~N} / \mathrm{A}$ & N/A & N/A & $\mathrm{N} / \mathrm{A}$ & N/A & N/A & N/A \\
\hline $\mathrm{ZrO} 2: 2 \mathrm{H} 2 \mathrm{O}$ & & $9.08 \mathrm{E}+01$ & $1.95 \mathrm{E}+00$ & & & & & $6.39 E+01$ \\
\hline Total & $1.59 E+07$ & $1.21 E+07$ & $1.28 \mathrm{E}+07$ & $1.57 \mathrm{E}+07$ & $1.31 \mathrm{E}+07$ & $1.41 \mathrm{E}+07$ & $7.92 \mathrm{E}+06$ & $2.18 \mathrm{E}+06$ \\
\hline
\end{tabular}


WHC-SD-WM-TI-784

Revision 0

Table A-1. Chemical inventory Estimates for Single-Shell Tanks

\begin{tabular}{|c|c|c|c|c|c|c|c|c|}
\hline $\begin{array}{l}\text { Soluble } \\
(\mathbf{k g})\end{array}$ & TY-102 & TY-103 & TY-104 & TY-105 & $T Y-106$ & U-101 & $\mathrm{U}-102$ & U-103 \\
\hline $\mathrm{Ag}+$ & N/A & $\mathbf{N} / \mathbf{A}$ & $\mathrm{N} / \mathrm{A}$ & N/A & $\mathrm{N} / \mathrm{A}$ & $N / A$ & N/A & $\mathrm{N} / \mathrm{A}$ \\
\hline Al(OH)4- & $2.18 \mathrm{E}+03$ & $2.63 E+03$ & & & & & $2.48 \mathrm{E}+04$ & $5.04 E+04$ \\
\hline $\mathrm{A} 8+5$ & N/A & N/A & $\mathrm{N} / \mathrm{A}$ & N/A & $\mathrm{N} / \mathrm{A}$ & N/A & N/A & N/A \\
\hline$B+3$ & N/A & $N / A$ & N/A & N/A & N/A & N/A & N/A & N/A \\
\hline $\mathrm{Ba}+2$ & N/A & N/A & $\mathrm{N} / \mathrm{A}$ & $\mathrm{N} / \mathrm{A}$ & N/A & N/A & N/A & N/A \\
\hline$B \theta+2$ & $\mathbf{N} / \mathbf{A}$ & $N / A$ & N/A & N/A & N/A & N/A & N/A & N/A \\
\hline $\mathrm{Bi}+3$ & $6.04 E+01$ & $3.46 E+01$ & & & & & $4.92 \mathrm{E}+01$ & $7.63 \mathrm{E}+01$ \\
\hline $\mathrm{Ca}+2$ & $2.29 E+01$ & $1.78 E+01$ & $5.96 \mathrm{E}-01$ & $2.19 E+0 O$ & 2.22E-01 & 8.41E-02 & $7.43 E+01$ & $7.65 E+01$ \\
\hline $\mathrm{Cd}+2$ & N/A & $\mathrm{N} / \mathrm{A}$ & N/A & N/A & N/A & N/A & N/A & N/A \\
\hline $\mathrm{Ce}+3$ & $4.42 E+00$ & $2.79 E+00$ & $2.29 \mathrm{E}+00$ & $2.82 \mathrm{E}-\mathrm{O} 2$ & & & $4.98 \mathrm{E}+01$ & $1.41 \mathrm{E}-04$ \\
\hline $\mathrm{Cl}-$ & $7.58 \mathrm{E}+02$ & $1.47 E+03$ & $4.86 \mathrm{E}+\mathrm{O} 2$ & $1.78 E+03$ & $1.81 E_{-02}$ & $8.60 E+00$ & $4.80 \mathrm{E}+03$ & $7.01 E+03$ \\
\hline $\mathrm{Co}+3$ & $N / A$ & $N / A$ & N/A & N/A & $N / A$ & $N / A$ & N/A & $\mathrm{N} / \mathrm{A}$ \\
\hline $\mathrm{co3}-2$ & $1.79 \mathrm{E}+03$ & $4.22 \mathrm{E}+03$ & $1.62 \mathrm{E}+03$ & $5.94 E+03$ & $6.02 E+02$ & $2.14 \mathrm{E}+03$ & $1.47 \mathrm{E}+04$ & $4.19 \mathrm{E}+04$ \\
\hline $\mathrm{Cr}(\mathrm{OH}) 4$ & $7.36 \mathrm{E}+02$ & $1.06 \mathrm{E}+03$ & $7.24 \mathrm{E}+00$ & $2.66 \mathrm{E}+01$ & $2.70 E-00$ & $2.66 \mathrm{E}+00$ & 4.73E + 03 & $3.75 E+03$ \\
\hline $\mathrm{Cs}+$ & $1.23 E+\infty 0$ & 2.47E + 00 & $2.81 E-02$ & $4.90 E-01$ & & $1.06 \mathrm{E}+00$ & $2.67 \mathrm{E}+00$ & 4.37E-04 \\
\hline $\mathrm{Cu}+2$ & N/A & $N / A$ & $\mathrm{~N} / \mathrm{A}$ & N/A & $\mathrm{N} / \mathrm{A}$ & N/A & N/A & $N / A$ \\
\hline F- & $2.80 \mathrm{E}+\mathrm{O3}$ & $3.35 \mathrm{E}+03$ & & & & & $2.28 \mathrm{E}+03$ & $3.54 \mathrm{E}+03$ \\
\hline $\mathrm{Fe}+3$ & $6.26 \mathrm{E}+01$ & $1.34 E+02$ & $5.37 E+01$ & $1.97 E+02$ & $2.00 E-01$ & $3.59 E+O 0$ & $2.54 \mathrm{E}+02$ & $2.14 E+02$ \\
\hline $\mathrm{H} 2 \mathrm{O}$ & $9.77 \mathrm{E}+05$ & $1.92 \mathrm{E}+06$ & $2.53 E+05$ & $9.31 E+05$ & $9.43 E-04$ & $2.31 \mathrm{E}+0.5$ & $5.14 \mathrm{E}+06$ & $6.58 E+06$ \\
\hline $\mathrm{Hg}+2$ & N/A & N/A & $\mathrm{N} / \mathrm{A}$ & N/A & $\mathrm{N} / \mathrm{A}$ & N/A & N/A & N/A \\
\hline $\mathrm{K}+$ & & & & & & & $4.08 \mathrm{E}+02$ & $1.54 E+03$ \\
\hline \multicolumn{9}{|l|}{$\mathrm{La}+3$} \\
\hline $\mathrm{Mg}+2$ & N/A & N/A & N/A & N/A & $N / A$ & N/A & N/A & $\mathrm{N} / \mathrm{A}$ \\
\hline $\mathrm{Mn}+4$ & & & & & & & $2.71 E+01$ & $1.02 E+02$ \\
\hline $\mathrm{Mo}+6$ & N/A & N/A & N/A & N/A & $\mathrm{N} / \mathrm{A}$ & N/A & N/A & N/A \\
\hline $\mathrm{Na}+$ & $1.29 E+05$ & $2.45 E+05$ & $3.24 E+04$ & $1.19 E+05$ & $1.21 E-0.4$ & $1.73 E+04$ & $6.89 E+05$ & $8.45 E+05$ \\
\hline $\mathrm{Ni}+3$ & $1.41 \mathrm{E}+01$ & $1.30 E+01$ & $8.92 E-01$ & $3.28 E+00$ & 3.32E.01 & $1.14 E-01$ & $4.75 \mathrm{E}+01$ & $4.73 \mathrm{E}+01$ \\
\hline NO2. & $8.70 \mathrm{E}+03$ & $1.22 \mathrm{E}+04$ & & & & & $9.14 \mathrm{E}+04$ & $1.46 \mathrm{E}+05$ \\
\hline No3- & $2.45 \mathrm{E}+05$ & $3.75 E+05$ & $4.84 \mathrm{E}+04$ & $1.78 \mathrm{E}+05$ & $1.80 E-04$ & $1.58 \mathrm{E}+03$ & $1.41 \mathrm{E}+06$ & $1.26 \mathrm{E}+06$ \\
\hline $\mathrm{OH}$. & $1.11 \mathrm{E}+04$ & $5.37 \mathrm{E}+04$ & $7.52 E+03$ & $2.76 E+04$ & $2.80 \mathrm{E}-03$ & $1.02 E+04$ & $6.83 E+04$ & $1.96 \mathrm{E}+05$ \\
\hline $\mathrm{Pb}+4$ & & & & & & & $6.12 \mathrm{E}-02$ & $2.31 \mathrm{E}-01$ \\
\hline PO4-3 & $1.91 E+04$ & $2.72 E+04$ & $6.98 \mathrm{E}+02$ & $2.57 E+03$ & $2.60 \mathrm{E}-02$ & $1.81 E+03$ & $1.57 E+04$ & $1.65 \mathrm{E}+04$ \\
\hline $\mathbf{R b}+$ & N/A & N/A & $\mathrm{N} / \mathrm{A}$ & N/A & $\mathrm{N} / \mathrm{A}$ & N/A & N/A & $\mathrm{N} / \mathrm{A}$ \\
\hline$R e+7$ & $N / A$ & $N / A$ & N/A & N/A & $N / A$ & $N / A$ & N/A & $N / A$ \\
\hline $\mathbf{R h}+\mathbf{3}$ & $N / A$ & $N / A$ & $\mathrm{~N} / \mathrm{A}$ & $\mathrm{N} / \mathrm{A}$ & $\mathrm{N} / \mathrm{A}$ & $N / A$ & $\mathrm{~N} / \mathrm{A}$ & $\mathrm{N} / \mathrm{A}$ \\
\hline $\mathbf{R u}+3$ & $N / A$ & N/A & $N / A$ & N/A & $N / A$ & N/A & $\mathrm{N} / \mathrm{A}$ & $\mathrm{N} / \mathrm{A}$ \\
\hline $\mathrm{Se}+6$ & $N / A$ & N/A & N/A & $N / A$ & N/A & $N / A$ & $\mathrm{~N} / \mathrm{A}$ & $\mathrm{N} / \mathrm{A}$ \\
\hline \multicolumn{9}{|l|}{$5 i+4$} \\
\hline so4-2 & $3.83 E+03$ & $1.02 \mathrm{E}+04$ & $5.73 E+03$ & $2.11 E+04$ & $2.13 \mathrm{E}-03$ & $2.70 E+03$ & $1.58 E+04$ & $1.75 \varepsilon+04$ \\
\hline $\mathrm{Sr}+2$ & $6.16 \mathrm{E}-04$ & 5.55E-03 & 3.14E-04 & $1.30 \mathrm{E}-02$ & 4.78E 04 & $6.82 \mathrm{E}-04$ & $2.61 \mathrm{E}-03$ & \\
\hline$T_{\theta}+6$ & N/A & N/A & N/A & N/A & $N / A$ & N/A & N/A & $\mathrm{N} / \mathrm{A}$ \\
\hline $\mathrm{Th}+4$ & $N / A$ & N/A & N/A & N/A & N/A & $N / A$ & N/A & $\mathrm{N} / \mathrm{A}$ \\
\hline $\mathrm{TI}+4$ & N/A & N/A & $N / A$ & N/A & N/A & N/A & $N / A$ & N/A \\
\hline TOC & $1.61 E+02$ & $1.61 \varepsilon+03$ & & & & & $4.12 \mathrm{E}+03$ & $9.00 E+03$ \\
\hline$\cup 02+2$ & $4.76 E+01$ & $5.36 \mathrm{E}+02$ & $3.28 \mathrm{E}+02$ & $1.20 E+03$ & $1.22 \mathrm{E}+\mathrm{O} 2$ & $1.22 \mathrm{E}+03$ & $2.78 E+03$ & $1.89 E+03$ \\
\hline $\mathrm{Zn}+2$ & N/A & $N / A$ & N/A & N/A & $\mathbf{N} / \mathbf{A}$ & N/A & $N / A$ & N/A \\
\hline $\mathrm{ZrO} 2: 2 \mathrm{H} 2 \mathrm{O}$ & & $4.17 E+01$ & & & & & & \\
\hline Total & $1.40 \mathrm{E}+06$ & $2.66 E+06$ & $3.51 \mathrm{E}+05$ & $1.29 \mathrm{E}+06$ & $1.31 \mathrm{E}+05$ & $2.68 \mathrm{E}+05$ & $7.49 \mathrm{E}+06$ & $9.18 \mathrm{E}+06$ \\
\hline
\end{tabular}


WHC-SD-WM-TI-784

Revision 0

Table A-1. Chemical Inventory Estimates; for Single-Shell Tanks

\begin{tabular}{|c|c|c|c|c|c|c|c|c|}
\hline $\begin{array}{c}\text { Soluble } \\
\text { (kg) }\end{array}$ & U.112 & U-201 & $\mathrm{U}-202$ & $U-203$ & U. 204 & TOTAL & $\begin{array}{c}\text { SALTWELL } \\
\text { LIO. PUMPED }\end{array}$ & NET TOTAL \\
\hline $\mathrm{Ag}+$ & $\mathrm{N} / \mathrm{A}$ & N/A & N/A & N/A & $\mathrm{N} / \mathrm{A}$ & $\mathrm{N} / \mathrm{A}$ & $\mathrm{N} / \mathrm{A}$ & N/A \\
\hline $\mathrm{Al}(\mathrm{OH}) 4$ & $3.68 \mathrm{E}+03$ & $8.29 E+02$ & $8.29 E+02$ & $4.15 E+02$ & $4.1 E E+02$ & $2.60 E+06$ & $5.36 E+05$ & $2.07 E+06$ \\
\hline $\mathrm{As}+5$ & N/A & N/A & N/A. & $N / A$ & $N / A$ & N/A & $\mathrm{N} / \mathrm{A}$ & N/A \\
\hline$B+3$ & $\mathrm{~N} / \mathrm{A}$ & $\mathrm{N} / \mathrm{A}$ & N/A & N/A & $N / A$ & $\mathrm{~N} / \mathrm{A}$ & N/A & $\mathrm{N} / \mathrm{A}$ \\
\hline $\mathrm{Ba}+2$ & $\mathrm{~N} / \mathrm{A}$ & N/A & N/A & $\mathrm{N} / \mathrm{A}$ & 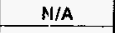 & N/A & N/A & N/A \\
\hline $\mathrm{Be}+2$ & N/A & N/A & $N / A$ & $\mathrm{~N} / \mathrm{A}$ & Al/A & N/A & N/A & N/A \\
\hline$B i+3$ & $9.24 E+00$ & & & & & $9.58 E+03$ & & $9.58 E+03$ \\
\hline $\mathrm{Ca}+2$ & $1.79 \mathrm{E}-01$ & 2.07E-02 & 2.07E-02 & $1.03 E-02$ & 1.0:3E-02 & $6.42 \mathrm{E}+03$ & & $6.42 E+03$ \\
\hline $\mathrm{Cd}+2$ & $\mathrm{~N} / \mathrm{A}$ & N/A & $\mathrm{N} / \mathrm{A}$ & $\mathrm{N} / \mathrm{A}$ & $\mathrm{N} / \mathrm{A}$ & N/A & N/A & N/A \\
\hline $\mathrm{C}_{e}+3$ & $3.51 E+01$ & $1.85 E+00$ & $1.84 \mathrm{E}+00$ & $1.62 \mathrm{E}+00$ & 3.71)E-01 & $2.35 E+03$ & & $2.35 E+03$ \\
\hline $\mathrm{Cl}-$ & $1.05 E+02$ & $5.94 E+00$ & $5.94 E+00$ & $2.97 \mathrm{E}+00$ & $2.97 E+\infty 0$ & $4.07 E+05$ & & $4.07 E+05$ \\
\hline $\mathrm{Co}+3$ & N/A & $\mathrm{N} / \mathrm{A}$ & N/A & $N / A$ & $N / A$ & N/A & N/A & $\mathrm{N} / \mathrm{A}$ \\
\hline CO3-2 & $2.77 \mathrm{E}+02$ & $3.19 E+01$ & $3.19 E+01$ & $1.60 E+01$ & $1.60 E+01$ & $1.62 \mathrm{E}+06$ & $4.70 E+04$ & $1.57 E+06$ \\
\hline $\mathrm{Cr}(\mathrm{OH}) 4$ & $1.01 E+02$ & $7.75 E-01$ & $7.75 \mathrm{E}-01$ & 3.87E-01 & 3.8''E-01 & $3.39 E+05$ & $2.71 E+04$ & $3.12 \mathrm{E}+05$ \\
\hline $\mathrm{Cs}+$ & $1.73 \mathrm{E}+00$ & $2.74 \mathrm{E}-01$ & 2.87E-01 & 2.53E-01 & $6.7: \mathrm{E}-02$ & $5.26 \mathrm{E}+\mathrm{O} 2$ & $1.85 \mathrm{E}+01$ & $5.08 \mathrm{E}+02$ \\
\hline $\mathrm{Cu}+2$ & $N / A$ & N/A & $\mathrm{N} / \mathrm{A}$ & $\mathrm{N} / \mathrm{A}$ & $\mathrm{N} / \mathrm{A}$ & N/A & $\mathrm{N} / \mathrm{A}$ & N/A \\
\hline F- & $1.33 E+03$ & & & & & $7.86 E+05$ & $6.38 \mathrm{E}+03$ & $7.79 E+05$ \\
\hline $\mathrm{Fe}+3$ & $1.15 E+01$ & $9.67 E-01$ & $9.67 E-01$ & 4.83E-01 & 4.8 & $2.37 \mathrm{E}+04$ & & $2.37 E+04$ \\
\hline $\mathrm{H} 2 \mathrm{O}$ & $4.58 \mathrm{E}+05$ & $8.28 \mathrm{E}+04$ & $8.28 \mathrm{E}+04$ & $4.23 E+04$ & $3.47 E+04$ & $4.05 E+08$ & $1.25 \mathrm{E}+07$ & $3.99 E+08$ \\
\hline $\mathrm{Hg}+2$ & $\mathrm{~N} / \mathrm{A}$ & N/A & N/A & $N / A$ & $\mathrm{~N} / \mathrm{A}$ & N/A & $\mathrm{N} / \mathrm{A}$ & $\mathrm{N} / \mathrm{A}$ \\
\hline $\mathrm{K}+$ & & & & & & $5.29 E+04$ & & $5.29 E+04$ \\
\hline $\mathrm{La}+3$ & & & & & & $1.88 \mathrm{E}+01$ & & $1.88 \mathrm{E}+07$ \\
\hline $\mathrm{Mg}+2$ & N/A & N/A & N/A & N/A & $\mathrm{N} / \mathrm{A}$ & $\mathrm{N} / \mathrm{A}$ & N/A & N/A \\
\hline $\mathrm{Mn}+4$ & & & & & & $2.41 E+03$ & & $2.41 E+03$ \\
\hline $\mathrm{Mo}+6$ & $\mathrm{~N} / \mathrm{A}$ & $\mathrm{N} / \mathrm{A}$ & N/A & N/A & N/A & $\mathrm{N} / \mathrm{A}$ & N/A & N/A \\
\hline $\mathrm{Na}+$ & $3.26 \mathrm{E}+04$ & $2.20 \mathrm{E}+03$ & $2.20 \mathrm{E}+03$ & $1.10 E+03$ & $1.10 \equiv+03$ & $5.68 \mathrm{E}+07$ & $3.31 E+06$ & $5.35 E+07$ \\
\hline $\mathrm{Ni}+3$ & 2.40E-01 & 2.99E-02 & $2.99 \mathrm{E}-02$ & 1.49E-02 & $1.4 \mathrm{~S} E-02$ & $4.06 \mathrm{E}+03$ & & $4.06 E+03$ \\
\hline NO2- & $2.15 E+03$ & $9.34 E+02$ & $9.34 E+02$ & $4.67 E+02$ & $4.671 \equiv+02$ & $6.45 \mathrm{E}+06$ & $1.48 \mathrm{E}+06$ & $4.97 E+06$ \\
\hline NO3- & $1.72 \mathrm{E}+04$ & $1.64 \mathrm{E}+03$ & $1.64 \mathrm{E}+03$ & $8.19 E+02$ & $8.19 \mathrm{l}+02$ & $9.87 E+07$ & $2.04 E+06$ & $9.67 E+07$ \\
\hline $\mathrm{OH}-$ & $1.33 \mathrm{E}+04$ & $7.15 \mathrm{E}+02$ & $7.15 \mathrm{E}+02$ & $3.58 \mathrm{E}+02$ & $3.55 \mathrm{I}+02$ & $9.25 E+06$ & $3.67 E+05$ & $9.25 E+06$ \\
\hline $\mathrm{Pb}+4$ & & & & & & $3.29 E+03$ & & $3.29 \mathrm{E}+03$ \\
\hline $\mathrm{PO} 4-3$ & $7.82 \mathrm{E}+03$ & & & & & $2.90 \mathrm{E}+06$ & $9.70 E+04$ & $2.81 \mathrm{E}+06$ \\
\hline $\mathrm{Rb}+$ & N/A & N/A & $\mathrm{N} / \mathrm{A}$ & $N / A$ & N'A & N/A & $N / A$ & N/A \\
\hline $\mathrm{Re}+7$ & $N / A$ & N/A & N/A & N/A & N'A & N/A & $\mathrm{N} / \mathrm{A}$ & N/A \\
\hline $\mathbf{R h}+\mathbf{3}$ & $N / A$ & N/A & $\mathrm{N} / \mathrm{A}$ & $\mathrm{N} / \mathrm{A}$ & N:A & N/A & N/A & N/A \\
\hline $\mathbf{R u}+3$ & N/A & N/A & $N / A$ & $\mathrm{~N} / \mathrm{A}$ & $\mathbf{N}$ 'A & N/A & $\mathrm{N} / \mathrm{A}$ & N/A \\
\hline $\mathrm{Se}_{\theta}+6$ & N/A & N/A & N/A & $\mathrm{N} / \mathrm{A}$ & N'A & N/A & $N / A$ & $\mathrm{~N} / \mathrm{A}$ \\
\hline $\mathrm{Si}+4$ & & & & & & $4.06 E+02$ & & $4.06 \mathrm{E}+02$ \\
\hline SO4-2 & $2.04 E+02$ & $1.80 \mathrm{E}+00$ & $1.80 E+00$ & 8.99E-01 & $8.99 \mathrm{E}-01$ & $1.63 E+06$ & & $1.63 \mathrm{E}+06$ \\
\hline $\mathrm{Sr}+2$ & $5.21 E-02$ & $3.46 \mathrm{E}-02$ & $3.46 E-02$ & $1.73 E-02$ & $1.73 \mathrm{E}-02$ & $3.63 E+02$ & $1.20 E+O O$ & $3.62 \mathrm{E}+\mathrm{O} 2$ \\
\hline $\mathrm{Te}+6$ & $\mathrm{~N} / \mathrm{A}$ & $N / A$ & N/A & N/A & $\mathrm{N}_{i}^{\prime} \mathrm{A}$ & N/A & N/A & N/A \\
\hline$T h+4$ & $\mathrm{~N} / \mathrm{A}$ & $\mathrm{N} / \mathrm{A}$ & N/A & N/A & NiA & N/A & N/A & $\mathrm{N} / \mathrm{A}$ \\
\hline$T I+4$ & N/A & N/A & N/A & N/A & NiA & N/A & $N / A$ & N/A \\
\hline TOC & & & & & & $4.58 \mathrm{E}+05$ & $2.16 \varepsilon+05$ & $2.26 E+05$ \\
\hline $\mathrm{UO} 2+2$ & $6.58 E+01$ & $4.00 E+01$ & $4.00 E+01$ & $2.00 \mathrm{E}+01$ & $3.11 \mathrm{E}-01$ & $9.67 E+04$ & & $9.67 E+04$ \\
\hline$Z n+2$ & N/A & N/A & $N / A$ & N/A & NiA & $N / A$ & $N / A$ & $N / A$ \\
\hline $\mathrm{ZrO} 2: 2 \mathrm{H} 2 \mathrm{O}$ & $1.03 \mathrm{E}+01$ & & & & & $6.68 \mathrm{E}+03$ & & $6.68 \mathrm{E}+03$ \\
\hline Total & $5.37 E+05$ & $8.92 E+04$ & $8.92 \mathrm{E}+04$ & $4.55 E+04$ & $3.78 \mathrm{E}+04$ & $6.47 \mathrm{E}+08$ & $2.07 E+07$ & $5.73 E+08$ \\
\hline
\end{tabular}


WHC-SD-WM-TI-784

Revision 0

Table A-1. Chemical Inventory Estimates for Single-Shell Tanks

\begin{tabular}{|c|c|c|c|c|c|c|c|c|}
\hline $\begin{array}{c}\text { Insoluble } \\
\text { (kg) }\end{array}$ & A-101 & A-102 & A-103 & A- 104 & A-105 & A-106 & AX-101 & $A X-102$ \\
\hline \multicolumn{9}{|l|}{ Agt } \\
\hline $\mathrm{Al}+3$ & $2.57 E+02$ & $8.64 E+00$ & $8.07 E+01$ & & & $8.53 E+00$ & $1.86 E+02$ & $6.51 E+02$ \\
\hline$A s+5$ & N/A & N/A & N/A & N/A & N/A & N/A & N/A & N/A \\
\hline$B+3$ & N/A & N/A & N/A & N/A & N/A & N/A & N/A & N/A \\
\hline $\mathrm{Ba}+2$ & $7.00 \mathrm{E}+01$ & $9.76 E+00$ & $1.20 \mathrm{E}+01$ & $1.87 E+01$ & 1.9BE-01 & $3.80 E+01$ & $1.44 \mathrm{E}+01$ & $1.92 E+00$ \\
\hline $8 \theta+2$ & N/A & N/A & N/A & N/A & N/A & N/A & N/A & N/A \\
\hline $\mathrm{Bi}+3$ & $3.04 E+00$ & $1.09 \mathrm{E}-01$ & $9.54 \mathrm{E}-01$ & & & $2.39 \mathrm{E}-01$ & $2.23 \mathrm{E}+00$ & 8.28E-O2 \\
\hline $\mathrm{Ca}+2$ & $1.12 \mathrm{E}+02$ & $3.27 E+01$ & $6.01 E+01$ & $3.42 E+02$ & $2.33 \varepsilon+02$ & $5.55 \mathrm{E}+02$ & $1.96 \mathrm{E}+02$ & $4.47 E+02$ \\
\hline Cancrinite & $5.37 E+04$ & $5.05 E+03$ & $2.96 \mathrm{E}+04$ & & & $6.44 E+04$ & $6.26 \mathrm{E}+04$ & $1.98 \mathrm{E}+03$ \\
\hline $\mathrm{Cd}+2$ & N/A & N/A & N/A & N/A & N/A & $\mathrm{N} / \mathrm{A}$ & N/A & $N / A$ \\
\hline $\mathrm{Ce}_{\theta}+3$ & & $1.51 E+03$ & $5.56 E+01$ & & $1.05 \mathrm{E}-03$ & & & \\
\hline $\mathrm{Cl}$ & $1.78 \mathrm{E}+\mathrm{O} 2$ & $7.72 \mathrm{E}+00$ & $6.52 \mathrm{E}+01$ & $3.70 E+00$ & 3. $15 \mathrm{E}-01$ & $3.00 E+01$ & $1.37 E+02$ & $6.26 \mathrm{E}+00$ \\
\hline $\mathrm{Co}+3$ & N/A & N/A & N/A & N/A & N/A & N/A & N/A & N/A \\
\hline $\cos -2$ & $5.32 \mathrm{E}+\mathrm{O} 2$ & $3.17 E+01$ & $1.85 E+02$ & $4.62 \mathrm{E}+01$ & $3.15 E+01$ & $2.00 E+02$ & $4.23 E+02$ & $7.53 E+01$ \\
\hline $\mathrm{Cr}+3$ & $2.23 E+01$ & 7.66E-01 & $7.72 \mathrm{E}+\mathrm{OO}$ & $1.14 \mathrm{E}+01$ & 7.07E-01 & $1.03 E+01$ & $1.59 E+01$ & $1.38 \mathrm{E}+00$ \\
\hline Cs+ & & $1.80 \mathrm{E}+00$ & 1.17E-01 & & 2. $15 \mathrm{E}-01$ & & & \\
\hline $\mathrm{Cu}+2$ & $\mathrm{~N} / \mathrm{A}$ & N/A & N/A & N/A & N/A & N/A & N/A & N/A \\
\hline F- & $1.52 \mathrm{E}+02$ & $5.47 \mathrm{E}+00$ & $4.90 \mathrm{E}+01$ & & & $1.20 \mathrm{E}+01$ & $1.12 \mathrm{E}+02$ & $4.22 \mathrm{E}+00$ \\
\hline $\mathrm{Fe}+3$ & $6.83 E+02$ & $1.19 E+02$ & $1.47 \mathrm{E}+02$ & $1.53 \mathrm{E}+03$ & $3.84 \mathrm{E}+03$ & $2.17 E+03$ & $2.15 E+03$ & $1.62 \mathrm{E}+03$ \\
\hline $\mathrm{Hg}+2$ & N/A & $N / A$ & N/A & N/A & N/A & N/A & N/A & N/A \\
\hline$K+$ & $2.90 E+01$ & $1.04 \mathrm{E}+00$ & $9.46 E+00$ & & & $2.28 \mathrm{E}+00$ & $2.15 E+01$ & $8.12 \mathrm{E} \cdot 01$ \\
\hline \multicolumn{9}{|l|}{$\mathrm{La}+3$} \\
\hline $\mathrm{Mg}+2$ & $N / A$ & N/A & N/A & N/A & $N / A$ & N/A & N/A & N/A \\
\hline $\mathrm{Mn}+4$ & $9.34 \mathrm{E}+02$ & $3.34 \mathrm{E}+01$ & $3.34 E+02$ & & & $7.36 E+01$ & $7.09 E+02$ & $2.80 E+01$ \\
\hline Mo+6 & N/A & N/A & N/A & $N / A$ & $\mathrm{~N} / \mathrm{A}$ & N/A & N/A & N/A \\
\hline $\mathrm{Na}+$ & $6.79 E+03$ & $3.46 \mathrm{E}+02$ & $2.63 E+03$ & $7.39 \mathrm{E}+02$ & $1.3 \mathrm{DE}+03$ & $1.98 \mathrm{E}+03$ & $5.96 \mathrm{E}+03$ & $2.46 \mathrm{E}+02$ \\
\hline $\mathrm{Ni}+3$ & $1.14 E+02$ & 6.87E-01 & $6.22 E+01$ & $5.06 \mathrm{E}+02$ & $3.42 \mathrm{E}+02$ & $3.72 \mathrm{E}+0.2$ & $1.97 \mathrm{E}+02$ & $9.69 E+01$ \\
\hline NO2- & $8.22 \mathrm{E}+02$ & $1.16 \mathrm{E}+02$ & & $1.51 \mathrm{E}-02$ & $3.12 E-02$ & $2.53 E+02$ & $2.37 E+03$ & $8.84 E+01$ \\
\hline NO3- & $2.38 \mathrm{E}+04$ & $8.48 \mathrm{E}+02$ & $9.15 \mathrm{E}+03$ & $8.80 E+01$ & $5.93 \mathrm{E}+00$ & $1.94 \mathrm{E}+03$ & $1.90 \mathrm{E}+04$ & $8.39 E+02$ \\
\hline $\mathrm{OH}-$ & & $6.26 E+02$ & & $3.95 \mathrm{E}+03$ & $7.4 .4 E+03$ & $3.01 E+03$ & $8.12 \mathrm{E}+02$ & $2.75 \mathrm{E}+\mathrm{O} 3$ \\
\hline $\mathrm{Pb}+4$ & $2.76 E+00$ & $9.91 E-02$ & $8.72 \mathrm{E}-01$ & & & 2.17E-01 & $2.03 E+00$ & $1.66 \mathrm{E}-01$ \\
\hline PO4-3 & $4.81 E+02$ & $1.73 \mathrm{E}+01$ & $1.66 \mathrm{E}+02$ & $1.25 E+02$ & & $1.35 \mathrm{E}+02$ & $3.54 E+02$ & $3.55 \mathrm{E}+01$ \\
\hline$R b+$ & N/A & N/A & N/A & N/A & W/A & N/A & N/A & $N / A$ \\
\hline $\mathrm{Ra}+7$ & N/A & N/A & N/A & N/A & $\mathrm{V} / \mathrm{A}$ & N/A & $\mathrm{N} / \mathrm{A}$ & N/A \\
\hline $\mathrm{Rh}+3$ & N/A & N/A & N/A & N/A. & $\mathrm{IN} / \mathrm{A}$ & N/A & N/A & N/A \\
\hline $\mathrm{Ru}+3$ & N/A & N/A & $\mathrm{N} / \mathrm{A}$ & N/A & $\mathrm{N} / \mathrm{A}$ & N/A & N/A & N/A \\
\hline $\mathrm{Se}+6$ & $N / A$ & N/A & N/A & N/A & IN/A & N/A & N/A & N/A \\
\hline $\mathrm{Si}+4$ & & & & $6.91 E+02$ & $5.80 \mathrm{E}+02$ & & & \\
\hline so4-2 & $5.12 \mathrm{E}+02$ & $1.88 \mathrm{E}+01$ & $2.47 E+02$ & $2.38 \mathrm{E}+00$ & $1.67 \mathrm{E}+02$ & $4.92 E+01$ & $5.13 E+02$ & $2.01 E+01$ \\
\hline$S r+2$ & $1.62 \mathrm{E}+01$ & $3.52 E+01$ & $9.52 \mathrm{E}-02$ & $1.91 E+01$ & $1.5 ! 5 \mathrm{E}+\infty 0$ & & 8.19E-01 & $9.36 \mathrm{E}-01$ \\
\hline Te+6 & $N / A$ & N/A & N/A & N/A & N/A & N/A & N/A & N/A \\
\hline$T h+4$ & N/A & N/A & N/A & N/A & IN/A & $\mathrm{N} / \mathrm{A}$ & N/A & N/A \\
\hline$T I+4$ & N/A & N/A & N/A & N/A & IN/A & N/A & N/A & N/A \\
\hline TOC & $3.72 \mathrm{E}+\mathrm{O} 2$ & $1.34 E+02$ & $1.17 E+02$ & & & $1.12 E+03$ & $3.97 E+02$ & $1.16 \mathrm{E}+01$ \\
\hline $\mathrm{UO} 2+2$ & $1.08 E+03$ & $1.64 E-01$ & $2.34 \mathrm{E}+00$ & $3.57 \mathrm{E}+02$ & $1.15 \mathrm{E}+04$ & $3.69 \mathrm{E}-01$ & $6.09 E+03$ & $1.82 \mathrm{E}-01$ \\
\hline $2 n+2$ & N/A & N/A & N/A & $N / A$ & IN/A & N/A & N/A & N/A \\
\hline \multicolumn{9}{|l|}{ 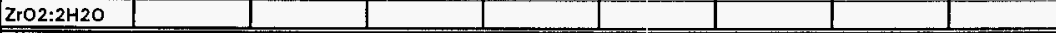 } \\
\hline Total & $9.06 \mathrm{E}+04$ & $8.95 E+03$ & 4.30E + 04 & $8.43 E+03$ & $2.55 \mathrm{E}+04$ & $7.64 \mathrm{E}+04$ & $1.02 \mathrm{E}+05$ & $8.91 E+03$ \\
\hline
\end{tabular}


Table A-1. Chemical Inventory Estimates for Single-Shell Tanks

\begin{tabular}{|c|c|c|c|c|c|c|c|c|}
\hline $\begin{array}{c}\text { Insoluble } \\
\text { (kg) } \\
\end{array}$ & $A X-103$ & AX-104 & B-101 & B-102 & E-103 & B-104 & 8-105 & B-106 \\
\hline \multicolumn{9}{|l|}{$A_{Q}+$} \\
\hline$A \mid+3$ & $1.74 E+01$ & & $2.76 E+03$ & $4.64 E+02$ & & $1.33 E+04$ & $3.90 \mathrm{E}+02$ & \\
\hline$A s+5$ & N/A & N/A & N/A & $N / A$ & $\mathrm{~N} / \mathrm{A}$ & N/A & N/A & N/A \\
\hline$B+3$ & N/A & N/A & N/A & N/A & N/A & N/A & N/A & N/A \\
\hline $\mathrm{Ba}+2$ & $1.04 \mathrm{E}-02$ & $2.50 E+01$ & $1.61 E+01$ & $1.23 E+00$ & 8.(11E-01 & $1.16 \mathrm{E}-01$ & $7.76 \mathrm{E}-01$ & $6.62 \mathrm{E}-02$ \\
\hline $\mathrm{Be}+2$ & $\mathrm{~N} / \mathrm{A}$ & $N / A$ & N/A & N/A & $N / A$ & $\mathrm{~N} / \mathrm{A}$ & $N / A$ & N/A \\
\hline$B i+3$ & 2.57E-01 & & $1.43 E+00$ & $4.59 \mathrm{E}-01$ & $1.07 E+00$ & $7.30 E+03$ & $9.33 E+02$ & $2.22 E+00$ \\
\hline $\mathrm{Ca}+2$ & $1.79 \mathrm{E}+02$ & $8.61 E+01$ & $1.03 E+03$ & $3.45 \varepsilon+01$ & $2.37 E+01$ & $1.31 E+03$ & $2.10 E+02$ & $1.89 \mathrm{E}+01$ \\
\hline Cancrinite & $3.03 E+04$ & & $3.83 E+04$ & $1.77 \mathrm{E}+03$ & $2.07 E+03$ & $4.48 E+04$ & $2.56 \mathrm{E}+04$ & $4.29 E+03$ \\
\hline $\mathrm{Cd}+2$ & N/A & $N / A$ & N/A & N/A & N/A & N/A & N/A & $N / A$ \\
\hline $\mathrm{Ce}_{\theta}+3$ & $1.75 E+02$ & $1.83 \mathrm{E}+00$ & & $2.62 \mathrm{E}+01$ & $9.013 E+01$ & $2.00 E+03$ & $1.48 \mathrm{E}+02$ & $1.73 E+01$ \\
\hline Cl- & $1.77 E+01$ & $1.16 \mathrm{E}-01$ & $2.02 E+01$ & $2.53 E+O O$ & $5.59 E+00$ & $3.58 \mathrm{E}+01$ & $3.13 E+01$ & $1.14 \mathrm{E}+01$ \\
\hline $\mathrm{Co}+3$ & N/A & N/A & N/A & N/A & IN/A & $\mathrm{N} / \mathrm{A}$ & $\mathrm{N} / \mathrm{A}$ & N/A \\
\hline $\cos -2$ & $7.19 \mathrm{E}+01$ & $1.16 E+01$ & $2.76 \mathrm{E}+02$ & $4.25 E+01$ & $5.07 E+01$ & $1.96 \mathrm{E}+02$ & & $3.77 E+01$ \\
\hline $\mathrm{Cr}_{\mathrm{r}+3}$ & $2.27 E+00$ & 2.61E-01 & $1.78 \mathrm{E}+00$ & $1.29 \mathrm{E}+00$ & $1.21 E+00$ & $8.52 E+01$ & $1.33 E+01$ & $1.02 \mathrm{E}+00$ \\
\hline $\mathrm{Cs}+$ & $1.03 \mathrm{E}-01$ & $6.97 \mathrm{E}-01$ & & 1.47E-02 & $5.95 E-02$ & $2.78 \mathrm{E}-02$ & $2.32 \mathrm{E}-01$ & $1.05 \mathrm{E}-02$ \\
\hline $\mathrm{Cu}+2$ & N/A & $N / A$ & N/A & N/A & IV/A & N/A & N/A & $N / A$ \\
\hline $\mathrm{F}-$ & $1.32 \varepsilon+01$ & & $6.66 \mathrm{E}+01$ & $2.13 E+01$ & $4.97 E+01$ & $5.83 E+02$ & $3.15 E+02$ & $1.03 E+02$ \\
\hline $\mathrm{Fe}+3$ & $2.84 E+03$ & $1.42 \mathrm{E}+03$ & $4.46 E+03$ & $1.16 E+02$ & $4.9: 3 E+01$ & $7.65 E+03$ & $9.69 E+02$ & $1.31 E+\infty 0$ \\
\hline $\mathrm{Hg}+2$ & N/A & N/A & N/A & $N / A$ & IV/A & N/A & N/A & $N / A$ \\
\hline$k+$ & $2.55 E+00$ & & & & & & & \\
\hline \multicolumn{9}{|l|}{$\operatorname{La}+3$} \\
\hline $\mathrm{Mg}+2$ & N/A & N/A & N/A & N/A & IN/A & N/A & N/A & $\mathrm{N} / \mathrm{A}$ \\
\hline$M n+4$ & $8.99 E+01$ & & & & & & & \\
\hline $\mathrm{Mo}+6$ & N/A & N/A & N/A & N/A & IN/A & $N / A$ & N/A & $N / A$ \\
\hline $\mathrm{Na}+$ & $1.60 \mathrm{E}+03$ & $4.81 \mathrm{E}+\mathrm{O2}$ & $1.58 E+03$ & $2.68 \mathrm{E}+02$ & 4.7.1E + 02 & $7.95 E+03$ & $2.85 E+03$ & $7.80 E+02$ \\
\hline $\mathrm{Ni}+3$ & $2.55 E+02$ & $1.26 E+02$ & $3.16 E+03$ & $4.43 E+01$ & 2.2:IE+01 & $1.72 \mathrm{E}+03$ & $2.34 \mathrm{E}+02$ & $7.26 \mathrm{E}+00$ \\
\hline NO2- & $2.75 E+02$ & $1.15 \mathrm{E}-02$ & $2.99 E+01$ & $9.82 \mathrm{E}+00$ & $2.19 \mathrm{E}+01$ & $5.27 E+01$ & & \\
\hline NO3- & $2.71 E+03$ & $2.19 E+00$ & $1.39 E+03$ & $3.91 \mathrm{E}+02$ & $9.015 E+02$ & $1.73 E+03$ & & $7.13 E+02$ \\
\hline $\mathrm{OH}$ & $4.09 E+03$ & $2.75 \mathrm{E}+03$ & $1.71 E+04$ & $1.21 \mathrm{E}+03$ & $1,0: \mathrm{E}+02$ & $8.14 E+02$ & & \\
\hline $\mathrm{Pb}+4$ & 2.35E-01 & & 5.51E-03 & & & & & \\
\hline PO4-3 & $4.09 \mathrm{E}+01$ & & $9.06 E+02$ & $5.22 E+02$ & 7.2 $\mathrm{JE}+02$ & $7.44 E+04$ & $1.12 E+04$ & $7.30 \mathrm{E}+\mathrm{O} 2$ \\
\hline $\mathbf{R b}+$ & N/A & N/A & N/A & N/A & IN/A & N/A & $N / A$ & N/A \\
\hline$R \theta+7$ & N/A & N/A & N/A & $\mathrm{N} / \mathrm{A}$ & IN/A & N/A & N/A & N/A \\
\hline$R h+3$ & N/A & N/A & N/A & $\mathrm{N} / \mathrm{A}$ & IN/A & N/A & N/A & N/A \\
\hline$R \mathbf{u}+\mathbf{3}$ & N/A & N/A & N/A & N/A & H/A & N/A & N/A & N/A \\
\hline $\mathrm{Se}+6$ & $N / A$ & N/A & N/A & N/A & W/A & $N / A$ & $N / A$ & N/A \\
\hline $\mathrm{Si}+4$ & & $2.14 E+02$ & & & 4.24E-01 & & & $7.72 \mathrm{E}-01$ \\
\hline SO4-2 & $1.90 E+02$ & $6.17 E+01$ & $8.11 E+01$ & $2.85 E+01$ & $5.6 \mathrm{EE}+01$ & $7.84 E+01$ & $2.47 \mathrm{E}+02$ & $1.01 E+02$ \\
\hline $\mathrm{Sr}+2$ & $1.24 \mathrm{E}+00$ & $4.61 E+01$ & & $1.60 \mathrm{E}-03$ & $4.81 \mathrm{E}-02$ & $3.96 \mathrm{E}-02$ & $5.94 \mathrm{E}-03$ & $7.81 \mathrm{E}-04$ \\
\hline $\mathrm{Te}+6$ & N/A & $N / A$ & $\mathrm{~N} / \mathrm{A}$ & $N / A$ & N/A & N/A & $N / A$ & N/A \\
\hline$T h+4$ & N/A & N/A & N/A & $N / A$ & N/A & $\mathrm{N} / \mathrm{A}$ & N/A & N/A \\
\hline $\mathrm{TI}+4$ & $\mathrm{~N} / \mathrm{A}$ & N/A & N/A & N/A & N/A & N/A & N/A & N/A \\
\hline TOC & $3.27 E+01$ & & $7.26 \mathrm{E}+01$ & & & & & \\
\hline $\mathrm{UO} 2+2$ & $8.51 E+03$ & $4.26 \mathrm{E}+03$ & $4.44 E+04$ & $3.39 E+03$ & $3.1 \mathrm{E} E+03$ & $6.01 E+01$ & $8,15 E+00$ & $1.12 \mathrm{E}-01$ \\
\hline$Z n+2$ & N/A & N/A & N/A & N/A & $\mathrm{N} / \mathrm{A}$ & N/A & N/A & N/A \\
\hline $\mathrm{ZrO} 2: 2 \mathrm{H} 2 \mathrm{O}$ & & & & & & $3.85 E+03$ & $5.10 E+02$ & \\
\hline Total & $5.15 \mathrm{E}+04$ & $9.49 E+03$ & $1.16 \mathrm{E}+05$ & $8.34 \mathrm{E}+03$ & $7.8 \mathrm{E}+03$ & $1.68 \mathrm{E}+05$ & $4.37 E+04$ & $6.81 \mathrm{E}+03$ \\
\hline
\end{tabular}


WHC-SD-WM-TI-784

Revision 0

Table A-1. Chemical Inventory Estimates for Single-Shell Tanks

\begin{tabular}{|c|c|c|c|c|c|c|c|c|}
\hline $\begin{array}{c}\text { Insoluble } \\
\left(\mathbf{k g}^{\prime}\right)\end{array}$ & B-107 & B-108 & B-109 & B-110 & 日-111 & B-112 & B-201 & B-202 \\
\hline $\mathrm{Ag}+$ & & & & & 3.:OE-05 & & & \\
\hline $\mathrm{Al}+3$ & $2.26 E+04$ & $4.19 E+03$ & $7.73 E+03$ & & & $1.82 \mathrm{E}+00$ & & \\
\hline $\mathrm{As}+5$ & $N / A$ & N/A & $\mathrm{N} / \mathrm{A}$ & $N / A$ & N/A & N/A & N/A & N/A \\
\hline$B+3$ & $N / A$ & N/A & N/A & $N / A$ & N/A & N/A & N/A & N/A \\
\hline $\mathrm{Ba}+2$ & $9.38 \mathrm{E}-01$ & $7.00 \mathrm{E}-01$ & 8.50E-01 & $1.65 E+01$ & $2.55 E+00$ & $6.74 E+00$ & & $1.12 \mathrm{E}-01$ \\
\hline $3 e+2$ & N/A & N/A & N/A & N/A & N/A & $\mathbf{N} / \mathbf{A}$ & $N / A$ & N/A \\
\hline$B i+3$ & $4.69 \mathrm{E}+03$ & $9.73 E+02$ & $1.61 E+00$ & $9.45 E+03$ & $8.69 E+03$ & $5.46 \mathrm{E}+\mathrm{O} 2$ & $1.05 E+03$ & $1.02 E+03$ \\
\hline $\mathrm{Ca}+2$ & $7.39 \mathrm{E}+02$ & $1.63 E+02$ & $2.20 \mathrm{E}+02$ & $1.90 E+03$ & $1.97 E+03$ & $1.11 E+02$ & $3.47 \mathrm{E}+02$ & $3.35 \mathrm{E}+\mathrm{O} 2$ \\
\hline Cancrinite & $2.23 E+04$ & $9.02 E+03$ & $6.17 E+03$ & & $1.09 \mathrm{E}+03$ & $4.98 \mathrm{E}+03$ & & \\
\hline$c d+2$ & N/A & N/A & $N / A$ & N/A & N/A & $\mathrm{N} / \mathrm{A}$ & $N / A$ & N/A \\
\hline $\mathrm{Ce}_{\theta}+3$ & $9.11 E+02$ & $1.83 E+02$ & $1.05 E+02$ & & $3.59 \mathrm{E}+00$ & $1.24 E+03$ & & \\
\hline $\mathrm{Cl}-$ & $9.89 E+01$ & $9.11 E+00$ & $9.32 \mathrm{E}+\mathrm{OO}$ & $4.53 E+01$ & 4.1 $3 E+01$ & $4.87 E+\infty 0$ & $5.92 \mathrm{E}+00$ & $5.72 \mathrm{E}+00$ \\
\hline $\mathrm{Co}+3$ & $N / A$ & N/A & N/A & N/A & N/A & N/A & N/A & $N / A$ \\
\hline $\mathrm{CO} 3-2$ & $9.96 \mathrm{E}+01$ & $4.01 E+01$ & $5.51 E+01$ & & & & $4.68 \mathrm{E}+01$ & $4.52 \mathrm{E}+01$ \\
\hline $\mathrm{Cr}+3$ & $6.34 E+01$ & $1.36 \mathrm{E}+01$ & $5.40 E+00$ & $9.32 E+01$ & $8.62 E+01$ & $6.26 \mathrm{E}+00$ & $1.22 \mathrm{E}+01$ & $1.18 \mathrm{E}+01$ \\
\hline $\mathrm{Cs}+$ & 2.71E-01 & $1.90 \mathrm{E}-01$ & $7.65 \mathrm{E}-02$ & $3.80 E-01$ & 3.3 $3 E+00$ & $1.39 E+00$ & & \\
\hline $\mathrm{Cu}+2$ & N/A & N/A & N/A & N/A & N/A & N/A & $N / A$ & N/A \\
\hline F- & $3.60 \mathrm{E}+02$ & $1.28 \mathrm{E}+02$ & $7.46 E+01$ & $6.49 \mathrm{E}+02$ & $5.9 .3 \mathrm{E}+\mathrm{O} 2$ & 3.97E+01 & $4.58 \mathrm{E}+\mathrm{O} 2$ & $4.43 E+02$ \\
\hline $\mathrm{Fe}+3$ & $4.16 \mathrm{E}+03$ & $8.63 E+02$ & $8.70 \mathrm{E}+\mathrm{O} 2$ & $1.17 \mathrm{E}+04$ & 1.3:3E+04 & $6.51 \mathrm{E}+02$ & $1.39 E+03$ & $1.34 E+03$ \\
\hline $\mathrm{Hg}+2$ & N/A & $\mathrm{N} / \mathrm{A}$ & N/A & N/A & N/A & $\mathrm{N} / \mathrm{A}$ & N/A & N/A \\
\hline$K+$ & & & & & & $3.45 \mathrm{E}-01$ & $1.53 E+02$ & $1.48 \mathrm{E}+02$ \\
\hline $\mathrm{La}+3$ & & & & & & & $9.62 \mathrm{E}+02$ & $1.56 \mathrm{E}+02$ \\
\hline $\mathrm{Mg}+2$ & N/A & N/A & N/A & $N / A$ & $\mathrm{~V} / \mathrm{A}$ & $\mathrm{N} / \mathrm{A}$ & N/A & $N / A$ \\
\hline$M n+4$ & & & & & & & $6.16 \mathrm{E}+03$ & $5.95 E+03$ \\
\hline $\mathrm{Mo}+6$ & N/A & N/A & N/A & N/A & $\mathrm{N} / \mathrm{A}$ & $\mathrm{N} / \mathrm{A}$ & N/A & $N / A$ \\
\hline $\mathrm{Na}+$ & $5.71 E+03$ & $1.60 \mathrm{E}+\mathrm{O} 3$ & $6.76 \mathrm{E}+\mathrm{O} 2$ & $8.65 E+03$ & $8.7 I E+03$ & $6.12 \mathrm{E}+02$ & $8.97 \mathrm{E}+02$ & 8.67E + 02 \\
\hline $\mathrm{Ni}+3$ & $9.09 E+02$ & $1.92 \mathrm{E}+02$ & $3.19 E+O 2$ & $2.60 \mathrm{E}+03$ & $2.61 E+03$ & $1.49 E+02$ & $5.05 E+02$ & $4.88 \mathrm{E}+02$ \\
\hline NO2- & $3.89 \mathrm{E}+01$ & $3.15 \mathrm{E}+01$ & $3.86 \mathrm{E}+01$ & & & & & \\
\hline NO3. & $3.60 E+02$ & $1.04 \mathrm{E}+03$ & $1.39 \mathrm{E}+03$ & & & & $2.14 \mathrm{E}+02$ & $2.07 E+02$ \\
\hline $\mathrm{OH}-$ & $2.01 E+04$ & $3.01 E+03$ & $1.57 \mathrm{E}+04$ & & & & $2.99 E+03$ & $2.89 E+03$ \\
\hline $\mathrm{Pb}+4$ & & & & & $1.11 \mathrm{E}-03$ & $4.36 \mathrm{E}-02$ & & \\
\hline PO4-3 & $6.02 E+04$ & $1.28 \mathrm{E}+04$ & $5.29 E+02$ & $7.27 \mathrm{E}+04$ & $6.613 \mathrm{E}+04$ & $4.21 E+03$ & $1.16 E+03$ & $1.12 \mathrm{E}+03$ \\
\hline $\mathrm{Rb}+$ & $N / A$ & N/A & N/A & $\mathrm{N} / \mathrm{A}$ & IN/A & N/A & N/A & $N / A$ \\
\hline $\operatorname{Re}+7$ & $N / A$ & N/A & N/A & $N / A$ & IN/A & $\mathrm{N} / \mathrm{A}$ & N/A & $N / A$ \\
\hline$R h+3$ & $N / A$ & N/A & N/A & $N / A$ & IN/A & N/A & $\mathrm{N} / \mathrm{A}$ & N/A \\
\hline$R u+3$ & N/A & N/A & $N / A$ & $N / A$ & IV/A & $\mathrm{N} / \mathrm{A}$ & N/A & N/A \\
\hline$S_{\theta}+6$ & N/A & $\mathrm{N} / \mathrm{A}$ & N/A & $N / A$ & IN/A & $N / A$ & N/A & N/A \\
\hline $\mathrm{Si}+4$ & & & & $1.13 E+03$ & $1.31 \mathrm{WE}+03$ & & & \\
\hline so4-2 & $1.95 \mathrm{E}+01$ & $5.65 E+01$ & $7.36 \mathrm{E}+01$ & $4.34 E+01$ & $1.3 ! \mathrm{E}+02$ & $1.41 E+01$ & $2.63 \mathrm{E}-01$ & 2.54E-01 \\
\hline $\mathrm{Sr}+2$ & $1.17 \mathrm{E}-01$ & $2.10 \mathrm{E}-01$ & $2.73 \mathrm{E}-02$ & 4.69E-01 & $1.0 \% \mathrm{E}+00$ & 6.60E-01 & $3.08 E+03$ & $2.97 E+03$ \\
\hline Te +6 & N/A & N/A & N/A & N/A & IV/A & N/A & N/A & $N / A$ \\
\hline Th+4 & N/A & $\mathrm{N} / \mathrm{A}$ & $N / A$ & $N / A$ & IN/A & N/A & N/A & $N / A$ \\
\hline $\mathrm{TI}+4$ & N/A & N/A & $N / A$ & $N / A$ & $I V / A$ & N/A & N/A & $N / A$ \\
\hline TOC & & & & & $2.72 \mathrm{E}-01$ & $1.66 E+00$ & $8.49 \varepsilon+01$ & $8.21 E+01$ \\
\hline $\mathrm{vO}_{2}+2$ & $7.31 E+01$ & $1.52 \mathrm{E}+01$ & $2.79 E+03$ & $1.23 E+03$ & $7.9 ! 5 \mathrm{E}+03$ & $1.09 E+00$ & & \\
\hline$Z n+2$ & N/A & N/A & $\mathrm{N} / \mathrm{A}$ & N/A & IV/A & $N / A$ & N/A & $N / A$ \\
\hline $\mathrm{ZrO} 2: 2 \mathrm{H} 2 \mathrm{O}$ & $5.21 E+03$ & $1.08 \mathrm{E}+03$ & & & & & & \\
\hline Total & $1.49 E+05$ & $3.55 E+04$ & $3.68 E+04$ & $1.10 \mathrm{E}+05$ & $1.13 \mathrm{E}+05$ & $1.26 E+04$ & $1.87 E+04$ & $1.81 E+04$ \\
\hline
\end{tabular}


Table A-1. Chemical Inventory Estimates for Single-Shell Tanks

\begin{tabular}{|c|c|c|c|c|c|c|c|c|}
\hline $\begin{array}{c}\text { Soluble } \\
(\mathbf{k g})\end{array}$ & U-104 & U-105 & $\mathrm{U}-108$ & $U-107$ & U-108 & U-109 & $U-110$ & U-111 \\
\hline $\mathrm{Ag}+$ & N/A & N/A & N/A & N/A & N/A & N/A & N/A & N/A \\
\hline $\mathrm{Al}(\mathrm{OH}) 4$ & & $3.80 E+04$ & $2.19 E+04$ & $4.38 E+04$ & $5.51 E+04$ & 5.27E + 04 & $4.62 \mathrm{E}+03$ & $3.88 \mathrm{E}+04$ \\
\hline$A s+5$ & $\mathrm{~N} / \mathrm{A}$ & N/A & N/A & N/A & $\mathrm{N} / \mathrm{A}$ & $N / A$ & N/A & N/A \\
\hline$B+3$ & N/A & N/A & $N / A$ & N/A & N/A & N/A & $N / A$ & N/A \\
\hline $\mathrm{Ba}+2$ & N/A & N/A & N/A & N/A & $N / A$ & N/A & N/A & N/A \\
\hline $\mathrm{Be}+2$ & N/A & N/A & N/A & N/A & N/A & N/A & N/A & $\mathrm{N} / \mathrm{A}$ \\
\hline $\mathrm{Bi}+3$ & & $7.69 E+01$ & $2.84 E+01$ & $7.25 E+01$ & $8.13 E+01$ & $7.79 \mathrm{E}+01$ & $2.85 \mathrm{E}+01$ & $4.28 \mathrm{E}+01$ \\
\hline $\mathrm{Ca}+2$ & $1.53 \mathrm{E}-01$ & $4.45 \mathrm{E}+01$ & $4.13 E+01$ & $2.15 E+01$ & $6.59 E+01$ & $5.72 \mathrm{E}+01$ & 4.7OE-O1 & $3.90 E+01$ \\
\hline $\mathrm{Cd}+2$ & N/A & $N / A$ & N/A & N/A & $\mathrm{N} / \mathrm{A}$ & N/A & N/A & N/A \\
\hline $\mathrm{Ce}_{\theta}+3$ & & & & $1.06 \mathrm{E}+01$ & $3.8: E-04$ & $9.70 \mathrm{E}-02$ & $6.16 \mathrm{E}-03$ & $2.85 E+00$ \\
\hline $\mathrm{Cl}-$ & $1.57 E+01$ & $6.44 E+03$ & $2.83 E+03$ & $5.91 \mathrm{E}+03$ & $7.23 E+03$ & $6.90 \mathrm{E}+03$ & $3.82 \mathrm{E}+\mathrm{O} 2$ & $5.60 E+03$ \\
\hline $\mathrm{Co}+3$ & N/A & N/A & N/A & N/A & N/A & N/A & N/A & $N / A$ \\
\hline CO3-2 & $3.91 E+03$ & $1,96 E+04$ & $2.24 E+04$ & $1.95 E+04$ & $3.54 E+04$ & $3.52 E+04$ & $1.55 E+03$ & $2.22 \mathrm{E}+04$ \\
\hline $\mathrm{Cr}(\mathrm{OH}) 4$ & $4.86 \mathrm{E}+00$ & $3.27 \mathrm{E}+03$ & $1.81 E+03$ & $2.20 \mathrm{E}+\mathrm{O} 3$ & $3.49 E+03$ & $3.31 E+03$ & $4.38 E+01$ & $2.54 \mathrm{E}+03$ \\
\hline $\mathrm{Cs}_{8}+$ & & $8.21 \mathrm{E}-04$ & & $1.50 \mathrm{E}+00$ & $1.1 \varepsilon \mathrm{E}-03$ & 3.67E-01 & $1.90 E-02$ & 4.00E-O1 \\
\hline $\mathrm{Cu}+2$ & N/A & $N / A$ & N/A & N/A & N/A & N/A & N/A & $N / A$ \\
\hline F. & & $3.57 \mathrm{E}+03$ & 1.32E + 03 & $3.37 \mathrm{E}+03$ & $3.77 E+03$ & $3.62 \mathrm{E}+03$ & $5.70 E+03$ & $3.42 \mathrm{E}+03$ \\
\hline $\mathrm{Fe}+3$ & $6.55 E+O 0$ & $1.43 E+02$ & $1.17 \mathrm{E}+02$ & $5.12 \mathrm{E}+01$ & 1.97E +02 & $9.88 \mathrm{E}+01$ & $2.65 \mathrm{E}+01$ & $9.22 \mathrm{E}+01$ \\
\hline $\mathrm{H} 2 \mathrm{O}$ & $4.21 E+05$ & $5.14 E+06$ & $3.08 \mathrm{E}+06$ & $4.51 E+06$ & $6.17 \equiv+06$ & $6.08 \mathrm{E}+08$ & $9.98 E+05$ & $4.42 E+06$ \\
\hline $\mathrm{Hg}+2$ & N/A & N/A & $N / A$ & $N / A$ & N/A & $N / A$ & N/A & $N / A$ \\
\hline$K+$ & & $1.13 E+03$ & $6.37 \mathrm{E}+02$ & $1.18 E+03$ & $1.56 \mathrm{E}+03$ & $1.48 E+03$ & & $1.17 \mathrm{E}+03$ \\
\hline \multicolumn{9}{|l|}{$\mathrm{La}+3$} \\
\hline $\mathrm{Mg}+2$ & N/A & N/A & $N / A$ & $N / A$ & N/A & N/A & N/A & $N / A$ \\
\hline $\mathrm{Mn}+4$ & & $7.54 \mathrm{E}+01$ & $4.24 E+01$ & $7.81 E+01$ & $1.03 \equiv+02$ & $9.87 E+01$ & & $7.80 E+01$ \\
\hline $\mathrm{Mo}+6$ & N/A & N/A & $N / A$ & $N / A$ & N/A & N/A & N/A & N/A \\
\hline $\mathrm{Na}+$ & $3.15 \mathrm{E}+04$ & B.62E + 05 & $3.98 \mathrm{E}+05$ & $5.68 \mathrm{E}+05$ & $7.93 \equiv+05$ & $7.77 E+05$ & $1.16 \mathrm{E}+05$ & $5.65 \mathrm{E}+05$ \\
\hline $\mathrm{Ni}+3$ & 2.08E-01 & $2.82 E+01$ & $2.55 \mathrm{E}+01$ & $1.07 E+01$ & $4.10 \mid=01$ & $3.01 E+01$ & $5.33 \mathrm{E}-01$ & $2.34 \mathrm{E}+01$ \\
\hline NO2- & & $1.42 \mathrm{E}+05$ & $5.56 E+04$ & $1.43 E+05$ & $1.55 \mid z+05$ & $1.53 E+05$ & $5.12 \mathrm{E}+03$ & $1.22 \mathrm{E}+05$ \\
\hline NO3- & $2.89 E+03$ & $9.73 E+05$ & $6.27 E+05$ & $6.71 E+05$ & $1.16 \equiv+06$ & $1.10 E+05$ & $5.04 E+04$ & $7.77 E+05$ \\
\hline $\mathrm{OH}$. & $1.86 E+04$ & $1.50 E+05$ & $8.66 E+04$ & $1.66 \mathrm{E}+05$ & $1.86 \mid+05$ & $1.92 E+05$ & $5.08 E+04$ & $1.43 E+05$ \\
\hline $\mathrm{Pb}+4$ & & $1.70 E-01$ & $9.57 \mathrm{E}-02$ & $1.77 \mathrm{E}-01$ & 2.34.E-01 & $2.23 \mathrm{E}-01$ & & $1.76 \mathrm{E}-01$ \\
\hline PO4-3 & $3.29 E+03$ & $1.83 E+04$ & $7.47 \mathrm{E}+03$ & $1.56 \mathrm{E}+04$ & $1.48 \equiv+04$ & $1.59 \mathrm{E}+04$ & $2.89 E+04$ & $1.26 \mathrm{E}+04$ \\
\hline $\mathbf{R b}+$ & N/A & N/A & N/A & N/A & $N / A$ & N/A & N/A & N/A \\
\hline $\operatorname{Re}+7$ & N/A & N/A & N/A & N/A & $N / A$ & N/A & N/A & N/A \\
\hline$R h+3$ & $N / A$ & N/A & N/A & N/A & N/A & N/A & N/A & N/A \\
\hline $\mathbf{R u}+\mathbf{3}$ & N/A & $N / A$ & N/A & N/A & $N / A$ & N/A & N/A & N/A \\
\hline$S e+6$ & N/A & $\mathrm{N} / \mathrm{A}$ & $N / A$ & N/A & N/A & N/A & N/A & N/A \\
\hline \multicolumn{9}{|l|}{$S i+4$} \\
\hline SO4-2 & $4.92 E+03$ & $1.46 \mathrm{E}+04$ & $9.39 E+03$ & $1.35 E+04$ & $1.351 \equiv+04$ & $1.54 E+04$ & $2.38 E+03$ & $9.42 E+03$ \\
\hline $5 r+2$ & & & & $3.33 \mathrm{E}-01$ & $2.16 \mathrm{E}-01$ & $2.08 \mathrm{E}-01$ & $1.58 \mathrm{E}-02$ & 2.04E-03 \\
\hline$T_{\theta}+6$ & N/A & N/A & N/A & $\mathrm{N} / \mathrm{A}$ & N/A & N/A & N/A & N/A \\
\hline $\mathrm{Th}+4$ & N/A & N/A & N/A & N/A & NIA & N/A & N/A & N/A \\
\hline$\pi 1+4$ & N/A & N/A & $\mathrm{N} / \mathrm{A}$ & N/A & $N / A$ & N/A & N/A & N/A \\
\hline TOC & & $8.69 E+03$ & $3.27 E+03$ & $8.60 E+03$ & $9.66 \mathrm{I}+03$ & $9.26 \mathrm{E}+03$ & & $7.66 \mathrm{E}+03$ \\
\hline $\mathrm{UO} 2+2$ & $2.23 E+03$ & $1.99 E+03$ & $1.50 E+03$ & $2.60 E+03$ & $6.361 \equiv+02$ & $1.68 E+03$ & $5.17 \mathrm{E}+\mathrm{O} 2$ & $9.31 \mathrm{E}+01$ \\
\hline$Z n+2$ & $N / A$ & N/A & N/A & N/A & N:A & N/A & N/A & $N / A$ \\
\hline $\mathrm{ZrO} 2: 2 \mathrm{H} 2 \mathrm{O}$ & & & & & & & $3.87 E+01$ & $3.85 E+00$ \\
\hline Total & $4.89 E+05$ & $7.19 \mathrm{E}+06$ & $4.32 E+06$ & $6.17 E+06$ & $8.611=06$ & $8.45 E+06$ & $1.27 E+06$ & $6.13 E+06$ \\
\hline
\end{tabular}


WHC-SD-WM-TI-784

Revision 0

Table A-1. Chemical Inventory Estimates for Single-Shell Tanks

\begin{tabular}{|c|c|c|c|c|c|c|c|c|}
\hline \multicolumn{9}{|l|}{$x$} \\
\hline $\begin{array}{c}\text { Insoluble } \\
(\mathrm{kg})\end{array}$ & B-203 & B-204 & $8 x-101$ & BX-102 & B: $<-103$ & $8 \times-104$ & $B X-105$ & $8 \times-106$ \\
\hline $\mathrm{Ag}+$ & & & & & & $3.45 E-05$ & $1.24 \mathrm{E}-05$ & 4.27E-05 \\
\hline $\mathrm{Al}+3$ & & & $1.10 E+03$ & $3.24 E+03$ & $5.2 \mathrm{OE}+03$ & & & \\
\hline As +5 & N/A & N/A & $\mathrm{N} / \mathrm{A}$ & N/A & $\mathrm{N} / \mathrm{A}$ & $\mathrm{N} / \mathrm{A}$ & N/A & N/A \\
\hline$B+3$ & N/A & N/A & N/A & N/A & $\mathrm{N} / \mathrm{A}$ & $\mathrm{N} / \mathrm{A}$ & N/A & N/A \\
\hline $\mathrm{Ba}+2$ & & & $4.64 \mathrm{E}-01$ & $1.31 \mathrm{E}+00$ & 6. $0 \mathrm{OE}-01$ & $3.36 \mathrm{E}+00$ & $1.57 \mathrm{E}+00$ & $1.63 E+01$ \\
\hline $\mathrm{Be}+2$ & $N / A$ & N/A & $\mathrm{N} / \mathrm{A}$ & N/A & $N / A$ & N/A & $N / A$ & N/A \\
\hline$B i+3$ & $1.88 \mathrm{E}+03$ & $1.84 \mathrm{E}+03$ & & & & & & \\
\hline $\mathrm{Ca}+2$ & $6.20 E+02$ & $6.08 \mathrm{E}+02$ & $3.91 \mathrm{E}+02$ & $2.5 O E+O 2$ & $3.91 \mathrm{E}+02$ & $2.00 \mathrm{E}+02$ & $2.24 \mathrm{E}+02$ & $1.51 E+02$ \\
\hline Cancrinite & & & $1.43 E+04$ & $6.50 E+01$ & $1.013 E+02$ & & & \\
\hline $\mathrm{Cd}+2$ & N/A & N/A & N/A & N/A & IN/A & $\mathrm{N} / \mathrm{A}$ & N/A & N/A \\
\hline $\mathrm{Ce}+3$ & & & & $7.64 E+02$ & & $4.43 \mathrm{E}-02$ & $6.27 E+02$ & $1.77 E+04$ \\
\hline $\mathrm{Cl}-$ & $1.06 \mathrm{E}+01$ & $1.04 E+01$ & $5.97 \mathrm{E}+00$ & $1.12 E+00$ & $1.7 ! 5 E+00$ & $8.48 \mathrm{E}-01$ & $9.49 \mathrm{E}-01$ & $6.41 \mathrm{E}-01$ \\
\hline $\mathrm{Co}+3$ & N/A & N/A & $N / A$ & $\mathrm{~N} / \mathrm{A}$ & IN/A & N/A & $N / A$ & N/A \\
\hline $\mathrm{cO}-2$ & $8.36 \mathrm{E}+01$ & $8.20 E+01$ & $3.85 E+02$ & $3.90 \mathrm{E}+02$ & $6.04 \mathrm{E}+02$ & $4.45 \mathrm{E}+02$ & $4.99 \mathrm{E}+\mathrm{O} 2$ & $3.37 E+02$ \\
\hline $\mathrm{Cr}+3$ & $2.17 E+01$ & $2.13 \mathrm{E}+01$ & $6.95 E+00$ & $1.02 \mathrm{E}+01$ & 1.5 $B E+01$ & $9.84 E+00$ & $1.10 E+01$ & $7.44 \mathrm{E}+00$ \\
\hline Cst & & & & $1.57 E+00$ & & $3.75 \mathrm{E}+00$ & 8.95E-01 & $3.35 \mathrm{E}+01$ \\
\hline $\mathrm{Cu}+2$ & N/A & N/A & N/A & N/A & IN/A & N/A & N/A & $\mathrm{N} / \mathrm{A}$ \\
\hline F- & $8.19 E+02$ & $8.03 E+02$ & & & & & & \\
\hline $\mathrm{Fe}+3$ & $2.48 \mathrm{E}+03$ & $2.44 \mathrm{E}+03$ & $1.74 E+03$ & $9.01 E+02$ & $1.41 E+03$ & $6.65 E+02$ & $7.45 E+02$ & $5.03 E+02$ \\
\hline $\mathrm{Hg}+2$ & $\mathrm{~N} / \mathrm{A}$ & $N / A$ & N/A & N/A & $\mathrm{N} / \mathrm{A}$ & N/A & N/A & N/A \\
\hline $\mathrm{K}+$ & $2.73 \mathrm{E}+02$ & $2.68 \mathrm{E}+02$ & & & & & & \\
\hline $\mathrm{La}+3$ & $2.89 \mathrm{E}+02$ & $2.83 \mathrm{E}+\mathrm{O} 2$ & & & & & & \\
\hline $\mathrm{Mg}+2$ & $\mathrm{~N} / \mathrm{A}$ & $N / A$ & N/A & N/A & $\mathrm{N} / \mathrm{A}$ & N/A & $\mathrm{N} / \mathrm{A}$ & N/A \\
\hline$M n+4$ & $1.10 E+04$ & $1.08 \mathrm{E}+04$ & & & & & & \\
\hline $\mathrm{Mo}+6$ & N/A & N/A & N/A & N/A & $\mathrm{N} / \mathrm{A}$ & $\mathrm{N} / \mathrm{A}$ & N/A & N/A \\
\hline $\mathrm{Ne}+$ & $1.60 \mathrm{E}+03$ & $1.57 E+03$ & $1.27 \mathrm{E}+03$ & $1.19 E+03$ & $1.84 E+03$ & $1.35 \mathrm{E}+03$ & $1.51 E+03$ & $1.02 \mathrm{E}+03$ \\
\hline $\mathrm{Ni}+3$ & $9.02 E+02$ & $8.85 E+02$ & $1.51 \mathrm{E}+03$ & $3.39 \mathrm{E}+02$ & $5.3 \mathrm{C} E+02$ & $2.56 E+02$ & $2.87 E+02$ & $1.94 E+02$ \\
\hline $\mathrm{NO}_{2-}$ & & & 2.14E-01 & $2.21 E+00$ & $3.55 E+00$ & & & \\
\hline NO3- & $3.83 \mathrm{E}+02$ & $3.76 \mathrm{E}+02$ & $9.74 \mathrm{E}+01$ & $4.15 \mathrm{E}+01$ & $6.51 \mathrm{E}+01$ & $2.99 E+01$ & $3.35 E+01$ & $2.27 \mathrm{E}+01$ \\
\hline $\mathrm{OH}-$ & $5.34 E+03$ & $5.23 E+03$ & $9.47 E+03$ & $1.06 E+04$ & $1.63 \mathrm{E}+04$ & $4.22 E+03$ & $4.96 \varepsilon+03$ & $9.64 E+03$ \\
\hline \multicolumn{9}{|l|}{$\mathrm{Pb}+4$} \\
\hline PO4-3 & $2.07 E+03$ & $2.03 E+03$ & $3.61 E+03$ & $4.32 E+03$ & $6.68 E+03$ & $5.07 \mathrm{E}+03$ & $5.68 \mathrm{E}+03$ & $3.84 E+03$ \\
\hline $\mathbf{R b}+$ & N/A & N/A & N/A & N/A & $\mathrm{N} / \mathrm{A}$ & N/A & N/A & $\mathrm{N} / \mathrm{A}$ \\
\hline$R e+7$ & N/A & N/A & $\mathrm{N} / \mathrm{A}$ & N/A & $N / A$ & N/A & N/A & $\mathrm{N} / \mathrm{A}$ \\
\hline $\mathrm{Rh}+3$ & N/A & N/A & N/A & N/A & $\mathrm{N} / \mathrm{A}$ & N/A & N/A & N/A \\
\hline $\mathbf{R u}+\mathbf{3}$ & $N / A$ & N/A & N/A & N/A & $\Lambda / A$ & $N / A$ & $N / A$ & N/A \\
\hline Se+6 & N/A & N/A & N/A & N/A & $\mathrm{N} / \mathrm{A}$ & $\mathrm{N} / \mathrm{A}$ & N/A & $\mathrm{N} / \mathrm{A}$ \\
\hline $\mathrm{Si}+4$ & & & & & & $1.39 \mathrm{E}+\infty \mathrm{O}$ & $1.56 \mathrm{E}+00$ & $1.05 E+00$ \\
\hline $504-2$ & 4.70E-01 & 4.61E-01 & $7.60 E+01$ & $8.80 E+01$ & $1.36 \mathrm{E}+02$ & $1.03 E+02$ & $1.15 E+02$ & $7.80 E+01$ \\
\hline $\mathrm{Sr}+2$ & $5.49 E+03$ & $5.39 E+03$ & & $6.25 \mathrm{E}-01$ & $1.22 \mathrm{E}+00$ & $9.17 \mathrm{E}-02$ & $1.97 E+01$ & $8.06 \mathrm{E}+00$ \\
\hline $\mathrm{Te}+6$ & N/A & $N / A$ & N/A & N/A & N/A & N/A & N/A & N/A \\
\hline$T h+4$ & N/A & N/A & $N / A$ & N/A & $\mathrm{N} / \mathrm{A}$ & N/A & N/A & N/A \\
\hline $\mathrm{TI}+4$ & N/A & N/A & N/A & N/A & $\mathrm{N} / \mathrm{A}$ & N/A & N/A & $N / A$ \\
\hline TOC & $1.52 E+02$ & $1.49 \mathrm{E}+02$ & $3.07 E+01$ & & & & & \\
\hline $\mathrm{UO} 2+2$ & & & $4.85 E+04$ & $3.81 E+04$ & $5.90 E+04$ & $4.34 E+04$ & $4.86 \mathrm{E}+04$ & $3.29 E+04$ \\
\hline $2 n+2$ & N/A & N/A & $N / A$ & $\mathrm{~N} / \mathrm{A}$ & $N / A$ & $\mathrm{~N} / \mathrm{A}$ & $\mathrm{N} / \mathrm{A}$ & N/A \\
\hline \multicolumn{9}{|l|}{$\mathrm{ZrO} 2: 2 \mathrm{H} 2 \mathrm{O}$} \\
\hline Total & $3.34 \mathrm{E}+04$ & $3.28 E+04$ & $8.25 E+04$ & $6.03 E+04$ & $9.22 E+04$ & $5.58 \mathrm{E}+04$ & $6.34 \mathrm{E}+04$ & $6.64 E+04$ \\
\hline
\end{tabular}


WHC-SD-WM-TI-784.

Revision 0

Table A-1. Chemical Inventory Estimates; for Single-Shell Tanks

\begin{tabular}{|c|c|c|c|c|c|c|c|c|}
\hline $\begin{array}{c}\text { Insoluble } \\
(\mathbf{k g})\end{array}$ & $B X-107$ & $B X-108$ & $B X-109$ & $B X-110$ & BK-111 & $B X-112$ & BY-101 & BY-102 \\
\hline \multicolumn{9}{|l|}{$\mathrm{Ag}+$} \\
\hline$A \mathrm{I}+3$ & $4.74 E+04$ & $3.58 \mathrm{E}+03$ & $2.20 E+04$ & $2.14 E+04$ & $4.44 \mathrm{E}+03$ & $1.79 E+04$ & $5.42 E+01$ & $4.84 \mathrm{E}+01$ \\
\hline $\mathrm{As}+5$ & N/A & N/A & N/A & $N / A$ & $\mathrm{~N} / \mathrm{A}$ & N/A & $\mathrm{N} / \mathrm{A}$ & N/A \\
\hline $8+3$ & N/A & N/A & $N / A$ & N/A & N/A & N/A & N/A & $N / A$ \\
\hline $\mathrm{Ba}+2$ & $4.12 \mathrm{E}-01$ & 2.47E-01 & $1.65 \mathrm{E}+00$ & $1.58 E+00$ & $1.41 \mathrm{E}+00$ & $2.36 \mathrm{E}-01$ & $4.23 E+00$ & $1.40 E+00$ \\
\hline $\mathrm{Ba}+2$ & N/A & $N / A$ & $N / A$ & N/A & N/A & N/A & N/A & $\mathrm{N} / \mathrm{A}$ \\
\hline $\mathrm{Bi}+3$ & $9.84 E+03$ & $7.45 \mathrm{E}+02$ & $4.57 E+03$ & $4.45 E+03$ & $9.1 \mathrm{BE}+02$ & $3.78 \mathrm{E}+03$ & $9.19 E-01$ & 8.21E-01 \\
\hline $\mathrm{Ca}+2$ & $1.55 E+03$ & $1.17 \mathrm{E}+\mathrm{O} 2$ & $1.39 \mathrm{E}+04$ & $7.07 \mathrm{E}+02$ & $1.73 E+02$ & $6.00 \mathrm{E}+02$ & $2.35 E+02$ & $1.36 E+02$ \\
\hline Cancrinite & $4.67 E+04$ & $3.54 E+03$ & $2.17 \mathrm{E}+04$ & $2.53 E+04$ & $2.21 E+04$ & $2.03 E+04$ & $3.47 E+04$ & $3.09 E+04$ \\
\hline $\mathrm{Cd}+2$ & N/A & N/A & N/A & N/A & N/A & $\mathrm{N} / \mathrm{A}$ & $N / A$ & N/A \\
\hline $\mathrm{C}_{\theta}+3$ & $3.20 \mathrm{E}+03$ & $7.06 \mathrm{E}+00$ & $4.68 \mathrm{E}-01$ & $1.96 \mathrm{E}+03$ & $2.01 E+03$ & $1.55 E+03$ & $2.58 \mathrm{E}+03$ & $7.47 E+02$ \\
\hline $\mathrm{Cl}-$ & $3.23 E+01$ & $2.44 E+00$ & $4.60 \mathrm{E}+02$ & $2.05 E+01$ & $2.83 \mathrm{E}+01$ & $1.55 \mathrm{E}+01$ & $5.00 \mathrm{E}+01$ & $4.43 E+01$ \\
\hline $\mathrm{Co}+3$ & N/A & $\mathrm{N} / \mathrm{A}$ & N/A & $N / A$ & $\mathrm{~N} / \mathrm{A}$ & $N / A$ & N/A & $\mathrm{N} / \mathrm{A}$ \\
\hline $\cos -2$ & $2.09 E+02$ & $1.58 \mathrm{E}+01$ & $3.22 E+03$ & $1.11 \mathrm{E}+02$ & $9.24 \mathrm{E}+01$ & $9.06 \mathrm{E}+01$ & $5.43 E+02$ & $3.22 \mathrm{E}+02$ \\
\hline $\mathrm{Cr}+3$ & $1.33 E+02$ & $1.01 E+01$ & $3.10 E+02$ & $6.23 E+01$ & $2.2 .2 E+01$ & $5.13 \mathrm{E}+01$ & $2.79 \mathrm{E}+01$ & $2.14 E+01$ \\
\hline $\mathrm{Cs}+$ & 8.22E-02 & $2.70 \mathrm{E}-03$ & $9.29 \mathrm{E}-\mathrm{O} 2$ & $2.21 E+00$ & $2.27 E+00$ & $4.11 \mathrm{E}-02$ & $2.91 \mathrm{E}+00$ & $8.44 \mathrm{E}-01$ \\
\hline $\mathrm{Cu}+2$ & N/A & $\mathrm{N} / \mathrm{A}$ & N/A & N/A & N/A & $\mathrm{N} / \mathrm{A}$ & N/A & $N / A$ \\
\hline F- & $7.54 \mathrm{E}+02$ & $5.71 E+01$ & $3.51 E+02$ & $3.47 E+02$ & $9.50 \mathrm{E}+01$ & $3.18 E+02$ & $4.78 E+01$ & $4.27 E+01$ \\
\hline $\mathrm{Fe}+3$ & $8.73 E+03$ & $6.61 E+02$ & $9.66 E+04$ & $3.95 E+03$ & $8.115 E+02$ & $3.35 \mathrm{E}+03$ & $6.03 E+02$ & $2.95 E+02$ \\
\hline $\mathrm{Hg}+2$ & N/A & N/A & N/A & N/A & IN/A & $N / A$ & N/A & N/A \\
\hline$k+$ & & & & $9.04 E-01$ & $3.83 \mathrm{E}+00$ & & $7.54 \mathrm{E}+00$ & $6.74 E+00$ \\
\hline \multicolumn{9}{|l|}{$\mathrm{La}+3$} \\
\hline $\mathrm{Mg}+2$ & $N / A$ & $\mathrm{~N} / \mathrm{A}$ & N/A & N/A & IN/A & $\mathrm{N} / \mathrm{A}$ & N/A & N/A \\
\hline \multicolumn{9}{|l|}{$M n+4$} \\
\hline $\mathrm{Mo}+6$ & N/A & N/A & N/A & N/A & IN/A & N/A & N/A & N/A \\
\hline $\mathrm{Na}+$ & $1.20 E+04$ & $9.06 E+02$ & $2.90 \mathrm{E}+04$ & $5.81 E+03$ & $2.65 E+03$ & $4.84 E+03$ & $4.14 E+03$ & $3.21 E+03$ \\
\hline $\mathrm{Ni}+3$ & $1.91 E+03$ & $1.44 \mathrm{E}+02$ & $1.95 E+04$ & $8.64 E+02$ & $1.89 \mathrm{E}+02$ & $7.33 E+02$ & $2.52 E+02$ & $1.31 E+02$ \\
\hline NO2- & $8.16 E+01$ & $6.18 E+00$ & $3.79 \mathrm{E}+01$ & $1.38 E+02$ & $4.42 \mathrm{E}+02$ & $4.38 E+01$ & $8.46 \mathrm{E}+02$ & $5.75 \mathrm{E}+02$ \\
\hline NO3- & $7.55 \mathrm{E}+02$ & $5.71 E+01$ & $8.85 \mathrm{E}+\mathrm{O} 3$ & $2.19 E+03$ & $7.99 \mathrm{E}+\mathrm{O} 3$ & $8.04 \mathrm{E}+02$ & $1.54 \mathrm{E}+04$ & $1.38 \mathrm{E}+04$ \\
\hline $\mathrm{OH}-$ & $4.27 \mathrm{E}+04$ & $3.15 \mathrm{E}+03$ & $1.23 \mathrm{E}+05$ & $1.92 \mathrm{E}+04$ & $3.3 \div \mathrm{E}+03$ & $1.59 E+04$ & $2.19 E+03$ & \\
\hline $\mathrm{Pb}+4$ & & & & $1.14 \mathrm{E}-01$ & $4.90 \mathrm{E}-01$ & & $9.53 \mathrm{E}-01$ & 8.51E-01 \\
\hline PO4-3 & $1.26 \mathrm{E}+05$ & $9.56 \mathrm{E}+03$ & $7.69 E+04$ & $5.71 E+04$ & $1.1 \leqq E+04$ & $4.86 E+04$ & $4.74 E+03$ & $2.39 \mathrm{E}+03$ \\
\hline $\mathrm{Rb}+$ & N/A & N/A & N/A & N/A & N/A & N/A & N/A & N/A \\
\hline$R \theta+7$ & N/A & $N / A$ & N/A & N/A & N/A & N/A & N/A & N/A \\
\hline $\mathrm{Rh}+3$ & N/A & N/A & N/A & N/A & N/A & $\mathrm{N} / \mathrm{A}$ & N/A & $\mathrm{N} / \mathrm{A}$ \\
\hline $\mathrm{Ru}+3$ & N/A & N/A & N/A & $N / A$ & $\mathrm{~N} / \mathrm{A}$ & $N / A$ & N/A & N/A \\
\hline Se +6 & N/A & N/A & N/A & N/A & $N / A$ & N/A & N/A & $\mathrm{N} / \mathrm{A}$ \\
\hline \multicolumn{9}{|l|}{$s i+4$} \\
\hline SO4-2 & $4.09 E+01$ & $3.09 E+00$ & $2.05 E+03$ & $5.14 E+01$ & $1.45 \mathrm{E}+\mathrm{O} 2$ & 4.37E + 01 & $3.68 E+02$ & $2.91 E+02$ \\
\hline $\mathrm{Sr}+2$ & $2.18 \mathrm{E}-01$ & $2.38 \mathrm{E}-02$ & $1.92 E+00$ & $1.07 E+00$ & $1.07 \mathrm{E}+00$ & $2.18 \mathrm{E}-02$ & $1.67 \mathrm{E}+00$ & $6.85 E \cdot 01$ \\
\hline $\mathrm{Te}+6$ & N/A & N/A & N/A & $\mathrm{N} / \mathrm{A}$ & $\mathrm{N} / \mathrm{A}$ & N/A & $\mathrm{N} / \mathrm{A}$ & N/A \\
\hline$T h+4$ & N/A & $N / A$ & $\mathrm{~N} / \mathrm{A}$ & N/A & $\mathrm{N} / \mathrm{A}$ & N/A & N/A & N/A \\
\hline $\mathrm{TI}+4$ & N/A & N/A & N/A & $N / A$ & $\mathrm{~N} / \mathrm{A}$ & N/A & N/A & $\mathrm{N} / \mathrm{A}$ \\
\hline TOC & & & & $4.14 E+00$ & $2.0 C E+01$ & & $3.97 E+01$ & $3.55 E+01$ \\
\hline $\mathrm{UO2}+2$ & $1.53 E+02$ & $1.16 \mathrm{E}+01$ & $2.30 E+04$ & $6.99 \mathrm{E}+01$ & $1.69 E+01$ & $5.88 E+01$ & $3.91 E+04$ & $1.91 E+04$ \\
\hline$Z n+2$ & N/A & N/A & $\mathrm{N} / \mathrm{A}$ & $\mathrm{N} / \mathrm{A}$ & $\mathrm{N} / \mathrm{A}$ & N/A & N/A & N/A \\
\hline $\mathrm{ZrO} 2: 2 \mathrm{H} 2 \mathrm{O}$ & $1.09 \mathrm{E}+04$ & $8.28 E+02$ & $5.08 \mathrm{E}+03$ & $4.94 E+03$ & $1.02 E+03$ & $4.19 E+03$ & & \\
\hline Total & $3.13 E+05$ & $2.34 E+04$ & $4.51 E+05$ & $1.49 E+05$ & $5.84 E+04$ & $1.23 E+05$ & $1.06 \mathrm{E}+05$ & $7.21 E+04$ \\
\hline
\end{tabular}


Table A-1. Chemical Inventory Estimates for Single-Shell Tanks

\begin{tabular}{|c|c|c|c|c|c|c|c|c|}
\hline $\begin{array}{c}\text { Insoluble } \\
(\mathbf{k g})\end{array}$ & BY-103 & BY-104 & BY-105 & BY-106 & BY-107 & BY-108 & BY-109 & BY-110 \\
\hline \multicolumn{9}{|l|}{$\mathrm{Ag}+$} \\
\hline A +3 & $5.35 E+03$ & $2.69 E+01$ & $4.02 E+01$ & $7.65 \mathrm{E}+01$ & $1.19 \mathrm{E}+02$ & & $6.01 \mathrm{E}+01$ & $3.62 \mathrm{E}+03$ \\
\hline As +5 & N/A & N/A & N/A & N/A & N/A & N/A & N/A & N/A \\
\hline$B+3$ & $\mathrm{~N} / \mathrm{A}$ & N/A & N/A & N/A & N/A & N/A & N/A & $N / A$ \\
\hline $\mathrm{Ba}+2$ & $2.75 E+00$ & $3.68 \mathrm{E}+00$ & $3.35 E+00$ & $2.76 \mathrm{E}+00$ & $1.87 E+00$ & $1.81 E+00$ & $1.25 E+00$ & $2.75 E+00$ \\
\hline$B \theta+2$ & N/A & N/A & N/A & N/A & N/A & N/A & N/A & N/A \\
\hline $\mathrm{Bi}+3$ & $1.03 E+00$ & $1.67 E+04$ & $1.58 \mathrm{E}+04$ & $1.01 E+04$ & $7.92 \mathrm{E}+03$ & $1.67 \mathrm{E}+04$ & $1.02 \mathrm{E}+00$ & $1.64 E+04$ \\
\hline $\mathrm{Ca}+2$ & $2.04 E+02$ & $2.39 E+03$ & $2.36 \mathrm{E}+03$ & $1.52 E+03$ & $1.76 E+03$ & $2.39 E+03$ & $2.36 E+02$ & $2.39 \mathrm{E}+03$ \\
\hline Cancrinite & $3.87 E+04$ & $9.11 \mathrm{E}+04$ & $-9.58 \mathrm{E}+04$ & $9.37 E+04$ & $4.62 \mathrm{E}+04$ & $5.66 \mathrm{E}+04$ & $3.84 E+04$ & $8.66 \mathrm{E}+04$ \\
\hline $\mathrm{Cd}+2$ & N/A & $\mathrm{N} / \mathrm{A}$ & N/A & N/A & $N / A$ & N/A & N/A & $N / A$ \\
\hline $\mathrm{Ce}+3$ & $1.34 E+02$ & $2.63 E+03$ & $2.04 E+03$ & $2.62 E+03$ & $9.55 E+02$ & $1.48 \mathrm{E}+03$ & $8.37 E+02$ & $2.34 E+03$ \\
\hline $\mathrm{Cl}-$ & $5.58 \mathrm{E}+01$ & $8.11 \mathrm{E}+01$ & $9.05 E+01$ & $1.09 E+02$ & $7.03 E+01$ & $6.44 E+01$ & $5.53 E+01$ & $8.47 E+01$ \\
\hline $\mathrm{Co}+3$ & N/A & N/A & N/A & N/A & N/A & N/A & N/A & N/A \\
\hline $\mathrm{CO}-2$ & $1.78 \mathrm{E}+02$ & $4.21 E+02$ & $6.12 E+02$ & $4.14 E+02$ & $3.55 E+02$ & $3.46 \mathrm{E}+02$ & $5.48 \mathrm{E}+02$ & $4.02 E+02$ \\
\hline $\mathrm{Cr}+3$ & $2.46 E+01$ & $4.49 \mathrm{E}+01$ & $5.16 E+01$ & $5.18 \mathrm{E}+01$ & $3.97 E+01$ & 4.16E + 01 & $2.98 \mathrm{E}+01$ & $6.13 E+01$ \\
\hline $\mathrm{Cs}+$ & $1.40 E-01$ & $2.97 E+00$ & $2.31 E+00$ & $2.95 E+00$ & $1.08 E+00$ & $1.39 E+\infty 0$ & $9.41 \mathrm{E}-01$ & $2.64 E+00$ \\
\hline $\mathrm{Cu}+2$ & N/A & N/A & N/A & N/A & N/A & N/A & N/A & N/A \\
\hline$F-$ & $5.35 \mathrm{E}+01$ & $4.52 E+02$ & $4.41 E+02$ & $3.34 E+02$ & $2.32 E+02$ & $4.42 \mathrm{E}+\mathrm{O} 2$ & $5.30 E+01$ & $5.10 E+02$ \\
\hline $\mathrm{Fe}+3$ & $6.06 E+02$ & $1.30 \mathrm{E}+04$ & $1.26 E+04$ & $8.05 E+03$ & 1.07E + 04 & $1.34 \mathrm{E}+04$ & $5.89 \varepsilon+02$ & $1.33 E+04$ \\
\hline $\mathrm{Hg}+2$ & N/A & N/A & $N / A$ & N/A & $N / A$ & N/A & N/A & N/A \\
\hline $\mathrm{K}+$ & $8.44 E+00$ & $5.53 \mathrm{E}+00$ & $7.29 E+00$ & $1.17 E+01$ & $3.22 \mathrm{E}+00$ & $1.36 \mathrm{E}+00$ & $8.36 E+00$ & $4.50 E+00$ \\
\hline \multicolumn{9}{|l|}{$L a+3$} \\
\hline $\mathrm{Mg}+2$ & $\mathrm{~N} / \mathrm{A}$ & N/A & N/A & $N / A$ & $\mathrm{~N} / \mathrm{A}$ & N/A & N/A & N/A \\
\hline \multicolumn{9}{|l|}{$\mathrm{Mn}+4$} \\
\hline $\mathrm{Mo}+6$ & N/A & N/A & $N / A$ & N/A & N/A & $N / A$ & N/A & N/A \\
\hline $\mathrm{Na}+$ & $3.37 E+03$ & $9.33 E+03$ & $1.02 E+04$ & $9.20 \mathrm{E}+03$ & $6.1 \mathrm{OE}+\mathrm{O} 3$ & $8.14 \mathrm{E}+03$ & $4.43 \mathrm{E}+\mathrm{O} 3$ & $1.01 \mathrm{E}+04$ \\
\hline $\mathrm{Ni}+3$ & $2.41 \mathrm{E}+02$ & $4.75 E+03$ & $4.61 E+03$ & $3.68 E+03$ & 4.3 $\mathrm{OE}+03$ & $6.38 \mathrm{E}+03$ & $2.48 \mathrm{E}+02$ & $6.21 E+03$ \\
\hline NO2- & $9.51 E+02$ & $6.20 \mathrm{E}+02$ & $8.17 E+02$ & $1.32 \mathrm{E}+03$ & $3.61 E+02$ & $1.53 E+02$ & $9.38 E+02$ & $5.13 E+02$ \\
\hline NO3. & $1.73 E+04$ & $1.22 E+04$ & $1.58 E+04$ & $2.46 \mathrm{E}+0.4$ & $7.53 E+03$ & $3.99 E+03$ & $1.71 E+04$ & $1.04 E+04$ \\
\hline $\mathrm{OH}-$ & $8.20 E+03$ & $1.58 \mathrm{E}+04$ & $1.58 \mathrm{E}+04$ & $7.76 \mathrm{E}+03$ & $1.34 \mathrm{E}+04$ & $1.87 E+04$ & $1.18 \mathrm{E}+\mathbf{0 3}$ & $1.77 E+04$ \\
\hline $\mathrm{Pb}+4$ & $1.07 \mathrm{E}+00$ & $6.98 \mathrm{E}-01$ & $9.20 \mathrm{E}-01$ & $1.48 E+00$ & 4.06E-01 & $1.72 \mathrm{E}-01$ & $1.06 \mathrm{E}+00$ & $5.68 \mathrm{E}-01$ \\
\hline PO4-3 & $2.01 E+02$ & $1.65 E+04$ & $1.77 E+04$ & $1.04 E+04$ & $9.42 E+03$ & $1.70 \mathrm{E}+04$ & $4.65 E+03$ & $2.94 E+04$ \\
\hline $\mathbf{R b}+$ & $\mathrm{N} / \mathrm{A}$ & N/A & N/A & N/A & N/A & N/A & $N / A$ & N/A \\
\hline $\operatorname{Re}+7$ & N/A & N/A & $\mathrm{N} / \mathrm{A}$ & N/A & $N / A$ & N/A & N/A & N/A \\
\hline$R h+3$ & N/A & N/A & $\mathrm{N} / \mathrm{A}$ & N/A & $\mathrm{N} / \mathrm{A}$ & $\mathrm{N} / \mathrm{A}$ & N/A & $N / A$ \\
\hline$R u+3$ & N/A & N/A & N/A & N/A & IN/A & N/A & $\mathrm{N} / \mathrm{A}$ & $N / A$ \\
\hline $\mathrm{Se}+6$ & N/A & N/A & $\mathrm{N} / \mathrm{A}$ & N/A & IN/A & $\mathrm{N} / \mathrm{A}$ & N/A & N/A \\
\hline $5 i+4$ & & & & & & $2.63 E+02$ & & \\
\hline so4-2 & $3.08 \mathrm{E}+02$ & $1.07 E+03$ & $1.13 \mathrm{E}+03$ & $9.56 \mathrm{E}+02$ & $6.213 \mathrm{E}+02$ & $9.27 E+02$ & $3.95 E+02$ & $9.77 E+02$ \\
\hline $\mathrm{Sr}+2$ & $3.43 \mathrm{E}+00$ & $1.40 E+00$ & $1.17 E+00$ & $1.78 E+00$ & $7.66 \mathrm{E}-01$ & $2.48 \mathrm{E}-01$ & $4.92 \mathrm{E}-01$ & $1.63 E+00$ \\
\hline $\mathrm{Te}+6$ & N/A & N/A & N/A & N/A & IN/A & $N / A$ & $N / A$ & $N / A$ \\
\hline$T h+4$ & N/A & N/A & N/A & N/A & IN/A & N/A & N/A & $N / A$ \\
\hline $\mathrm{Tl}+4$ & N/A & N/A & N/A & N/A & IN/A & N/A & N/A & N/A \\
\hline TOC & 4.47E+01 & $5.41 E+02$ & $5.23 \mathrm{E}+02$ & $5.39 E+02$ & $5.00 \mathrm{E}+02$ & $8.49 E+02$ & $4.40 E+01$ & $8.20 E+02$ \\
\hline $\mathrm{UO} 2+2$ & $1.94 \mathrm{E}+03$ & $1.91 E+04$ & $3.51 E+04$ & $1.16 \mathrm{E}+04$ & $1.40 \mathrm{E}+04$ & $1.92 E+04$ & $3.82 E+04$ & $1.77 E+04$ \\
\hline$Z n+2$ & $\mathrm{~N} / \mathrm{A}$ & N/A & N/A & N/A & IN/A & N/A & N/A & N/A. \\
\hline $\mathrm{ZrO} 2: 2 \mathrm{H} 2 \mathrm{O}$ & & & & & $3.18 \mathrm{E}+01$ & & & $1.18 \mathrm{E}+03$ \\
\hline Total & $7.79 E+04$ & $2.07 \mathrm{E}+0.5$ & $2.32 \mathrm{E}+0.05$ & $1.87 \mathrm{E}+05$ & $1.25 \mathrm{E}+05$ & $1.67 E+05$ & $1.08 E+05$ & $2.21 \mathrm{E}+05$ \\
\hline
\end{tabular}


WHC-SD-WM-TI-784

Revision 0

Table A-1. Chemical Inventory Estimates for Single-Shell Tanks

\begin{tabular}{|c|c|c|c|c|c|c|c|c|}
\hline $\begin{array}{c}\text { Insoluble } \\
(\mathbf{k g})\end{array}$ & $B Y-111$ & BY-112 & C-101 & C. 102 & C. 103 & C-104 & C-105 & C- 106 \\
\hline \multicolumn{9}{|l|}{$\mathrm{Ag}+$} \\
\hline$A l+3$ & $6.73 E+01$ & $4.20 E+01$ & $2.21 E+04$ & $2.28 \mathrm{E}+05$ & $2.0 \% \mathrm{E}+04$ & $1.10 E+05$ & $8.13 E+04$ & $8.09 E+03$ \\
\hline$A s+5$ & $\mathrm{~N} / \mathrm{A}$ & N/A & $N / A$ & N/A & IN/A & $N / A$ & N/A & N/A \\
\hline$B+3$ & N/A & $\mathrm{N} / \mathrm{A}$ & $\mathrm{N} / \mathrm{A}$ & N/A & N $/ A$ & N/A & N/A & N/A \\
\hline $\mathrm{Ba}+2$ & $2.32 E+00$ & $1.58 E+00$ & $1.50 E+01$ & $3.46 \mathrm{E}-01$ & $1.4 \$ E+01$ & $1.10 E+00$ & $4.53 E+01$ & $1.62 \mathrm{E}+01$ \\
\hline $\mathrm{Be}+2$ & $N / A$ & N/A & N/A & $\mathbf{N} / \mathbf{A}$ & IN/A & N/A & N/A & N/A \\
\hline $\mathrm{Bi}+3$ & $1.14 E+00$ & $6.69 \mathrm{E}+02$ & & & & & & \\
\hline $\mathrm{Ca}+2$ & $1.95 E+02$ & $2.54 E+02$ & $1.48 \mathrm{E}+03$ & $6.15 \mathrm{E}+03$ & $8.90 E+02$ & $3.97 E+03$ & $1.65 E+03$ & $2.65 E+03$ \\
\hline Cancrinite & $4.30 E+04$ & $2.98 E+04$ & $1.86 \mathrm{E}+00$ & $6.41 E+0.2$ & 3.2: $\mathrm{V}+04$ & $5.57 \varepsilon+04$ & $1.46 \mathrm{E}+03$ & $1.73 E+05$ \\
\hline $\mathrm{Cd}+2$ & N/A & $N / A$ & N/A & N/A & IN/A & N/A & N/A & N/A \\
\hline $\mathrm{Ce}+3$ & $2.64 \mathrm{E}+03$ & $4.84 E+02$ & $1.64 \mathrm{E}-01$ & & & & $7.09 \mathrm{E}-03$ & \\
\hline $\mathrm{Cl}-$ & $6.16 \mathrm{EE}+01$ & $4.35 E+01$ & $3.42 E+01$ & $5.67 \mathrm{E}+01$ & 6.2 $1 E+00$ & $5.71 E+01$ & 1.77E + 01 & $4.89 E+01$ \\
\hline $\mathrm{Co}+3$ & $\mathrm{~N} / \mathrm{A}$ & $N / A$ & $N / A$ & $N / A$ & N/A & N/A & N/A & $\mathrm{N} / \mathrm{A}$ \\
\hline CO3-2 & $4.59 E+02$ & $2.10 E+02$ & $3.05 E+02$ & $9.32 E+02$ & $1.20 E+02$ & $6.93 E+02$ & $2.50 \mathrm{E}+\mathrm{O} 2$ & $5.35 E+02$ \\
\hline $\mathrm{Cr}+3$ & $2.99 \mathrm{E}+01$ & $1.93 E+01$ & $3.01 E+01$ & $1.53 E+02$ & $2.35 E+01$ & $8.36 \mathrm{E}+01$ & $5.62 \mathrm{E}+01$ & $5.91 \varepsilon+01$ \\
\hline Cs + & $2.98 \mathrm{E}+00$ & 5.47E-01 & $1.02 E+00$ & & & & $1.56 \mathrm{E}+00$ & \\
\hline $\mathrm{Cu}+2$ & N/A & N/A & N/A & $N / A$ & N/A & N/A & N/A & $\mathrm{N} / \mathrm{A}$ \\
\hline F. & $5.93 \mathrm{E}+01$ & $5.42 \mathrm{E}+01$ & & $1.04 \mathrm{E}+03$ & & $2.13 E+03$ & & \\
\hline $\mathrm{Fe}+3$ & 4.27E + 02 & $1.24 E+03$ & $8.85 E+03$ & $2.76 \mathrm{E}+04$ & $3.70 \mathrm{E}+03$ & $1.72 \mathrm{E}+04$ & $7.41 E+03$ & $1.30 E+04$ \\
\hline $\mathrm{Hg}+2$ & N/A & N/A & $N / A$ & $N / A$ & N/A & N/A & N/A & N/A \\
\hline$K+$ & $9.36 \mathrm{E}+00$ & $5.92 E+00$ & & $5.14 E+01$ & & $9.67 \mathrm{E}+01$ & & \\
\hline \multicolumn{9}{|l|}{$\mathrm{La}+3$} \\
\hline $\mathrm{Mg}+2$ & N/A & N/A & $\mathrm{N} / \mathrm{A}$ & $\mathrm{N} / \mathrm{A}$ & N/A & N/A & N/A & $N / A$ \\
\hline \multicolumn{9}{|l|}{$M n+4$} \\
\hline Mo +6 & N/A & N/A & $N / A$ & N/A & N/A & N/A & N/A & N/A \\
\hline $\mathrm{Ne}+$ & $4.50 E+03$ & $2.95 E+03$ & $2.00 \mathrm{E}+03$ & $6.21 \mathrm{E}+03$ & $6.61 E+02$ & $6.34 E+03$ & $2.07 E+03$ & $3.68 \mathrm{E}+03$ \\
\hline $\mathrm{Ni}+3$ & $1.89 \mathrm{E}+02$ & $3.68 \mathrm{E}+02$ & $2.15 \mathrm{E}+03$ & $9.22 \mathrm{E}+03$ & $1.34 \mathrm{E}+03$ & $5.18 E+03$ & $2.40 E+03$ & $7.24 E+03$ \\
\hline No2. & $1.05 \mathrm{E}+03$ & $6.64 \mathrm{E}+02$ & $1.51 \mathrm{E}+01$ & $1.88 \mathrm{E}+02$ & $1.6 \mathrm{E} E+01$ & $1.23 E+02$ & $1.33 E+02$ & $4.65 E+01$ \\
\hline NO3- & $1.91 \mathrm{E}+04$ & $1.22 E+04$ & $7.13 E+02$ & $1.85 \mathrm{E}+03$ & 1.4 $\bar{O} E+02$ & $1.17 \mathrm{E}+03$ & $6.28 E+02$ & $7.86 \mathrm{E}+02$ \\
\hline $\mathrm{OH}-$ & 4.77E + 02 & $2.34 E+02$ & $5.39 E+04$ & $4.75 E+05$ & 4.4SE +04 & $2.35 \mathrm{E}+05$ & $1.66 \mathrm{E}+05$ & $4.25 E+04$ \\
\hline $\mathrm{Pb}+4$ & $1.18 \mathrm{E}+00$ & 7.48E-01 & & & & 4.22E-01 & & \\
\hline PO4-3 & $3.44 E+03$ & $1.66 E+03$ & $1.42 E+03$ & $1.88 E+03$ & $1.11 E+02$ & $6.60 \mathrm{E}+02$ & $3.65 E+02$ & $1.17 \mathrm{E}+03$ \\
\hline $\mathrm{Rb}+$ & N/A & N/A & N/A & N/A & $N / A$ & N/A & $N / A$ & N/A \\
\hline$R e+7$ & N/A & N/A & N/A & N/A & $\mathrm{N} / \mathrm{A}$ & N/A & N/A & N/A \\
\hline $\mathrm{Rh}+3$ & $\mathrm{~N} / \mathrm{A}$ & N/A & N/A & N/A & $\mathrm{N} / \mathrm{A}$ & N/A & $\mathrm{N} / \mathrm{A}$ & N/A \\
\hline$R u+3$ & N/A & N/A & N/A & N/A & $\mathrm{N} / \mathrm{A}$ & N/A & N/A & $N / A$ \\
\hline Se +6 & N/A & N/A & N/A & N/A & $\mathrm{N} / \mathrm{A}$ & N/A & N/A & N/A \\
\hline \multicolumn{9}{|l|}{$\mathrm{Si}+4$} \\
\hline so4-2 & $4.06 \mathrm{E}+02$ & $2.79 E+02$ & $1.47 E+02$ & $9.57 E+01$ & $1.7 E E+O O$ & $1.92 E+01$ & $4.20 E+01$ & $9.01 \mathrm{E}+01$ \\
\hline $\mathrm{Sr}+2$ & $1.64 \mathrm{E}+00$ & $3.72 \mathrm{E}-01$ & $3.44 \mathrm{E}-01$ & $5.14 \mathrm{E}+00$ & $1.99 E+00$ & $1.22 E+01$ & $1.41 \mathrm{E}+01$ & $2.70 E+O O$ \\
\hline $\mathrm{Te}+6$ & N/A & N/A & $N / A$ & $\mathrm{~N} / \mathrm{A}$ & $\mathrm{N} / \mathrm{A}$ & N/A & $\mathrm{N} / \mathrm{A}$ & N/A \\
\hline$T h+4$ & N/A & N/A & N/A & N/A & N/A & N/A & N/A & N/A \\
\hline $\mathrm{TI}+4$ & N/A & N/A & N/A & N/A & $\mathrm{N} / \mathrm{A}$ & N/A & N/A & N/A \\
\hline TOC & $4.94 E+01$ & $5.18 E+01$ & & $5.05 E-01$ & & $1.97 E+03$ & & $9.51 E+01$ \\
\hline $\mathrm{UO} 2+2$ & $2.76 \mathrm{E}+04$ & $7.85 E+03$ & $1.60 E+04$ & $8.10 E+04$ & $8.17 E+03$ & $4.07 E+04$ & $2.21 E+04$ & $7.03 E+04$ \\
\hline$Z n+2$ & N/A & N/A & N/A & N/A & N/A & N/A & N/A & N/A \\
\hline $\mathrm{ZrO} 2: 2 \mathrm{H} 2 \mathrm{O}$ & & & & $9.75 E+04$ & & $2.02 E+05$ & & \\
\hline Total & $1.04 E+05$ & $5.91 E+04$ & $1.09 E+05$ & $9.37 E+05$ & $1.14 E+05$ & $6.83 E+05$ & $2.86 \mathrm{E}+05$ & $3.23 E+05$ \\
\hline
\end{tabular}


WHC-SD-WM-TI-784

Revision 0

Table A-1. Chemical Inventory Estimates for Single-Shell Tanks

\begin{tabular}{|c|c|c|c|c|c|c|c|c|}
\hline $\begin{array}{c}\text { Insoluble } \\
\text { (kg) }\end{array}$ & C-107 & C-108 & C- 109 & $C-110$ & C.111 & C-112 & $\mathrm{C}-201$ & $c-202$ \\
\hline \multicolumn{9}{|l|}{$\mathbf{A g}+$} \\
\hline $\mathrm{Al}+3$ & $2.99 E+04$ & $2.48 \mathrm{E}+0.3$ & $1.38 \mathrm{E}+03$ & $2.57 E+04$ & $1.41 E+04$ & $1.25 E+04$ & & \\
\hline $\mathrm{As}+5$ & N/A & N/A & N/A & N/A & IV/A & N/A & N/A & N/A \\
\hline$B+3$ & N/A & N/A & N/A & N/A & $I V / A$ & $\mathrm{~N} / \mathrm{A}$ & N/A & N/A \\
\hline $\mathrm{Ba}+2$. & 2.31E-01 & 4.60E-01 & $6.15 E-01$ & $7.57 \mathrm{E}-02$ & $5.84 E-01$ & $3.06 \mathrm{E}-01$ & 8.80E-04 & $1.08 \mathrm{E}-02$ \\
\hline $\mathrm{Be}+2$ & N/A & $\mathbf{N} / \mathbf{A}$ & N/A & N/A & IV/A & N/A & N/A & $\mathrm{N} / \mathrm{A}$ \\
\hline $\mathrm{Bi}+3$ & $7.02 E+03$ & $5.15 E+02$ & $2.86 E+02$ & $5.35 E+03$ & $1.03 E+03$ & $4.29 E+02$ & & \\
\hline $\mathrm{Ca}+2$ & $1.40 \mathrm{E}+03$ & $1.01 E+03$ & $2.09 E+03$ & $8.42 E+02$ & $4.9 .4 \mathrm{E}+02$ & $3.50 \mathrm{E}+03$ & $9.75 \mathrm{E}+00$ & $4.87 E+00$ \\
\hline Cancrinite & $6.50 E+04$ & $2.45 \mathrm{E}+03$ & $1.36 E+03$ & $2.54 E+04$ & $5.17 \mathrm{E}+03$ & $2.04 E+03$ & & \\
\hline $\mathrm{Cd}+2$ & N/A & N/A & N/A & N/A & $\mathbf{I N} / \mathbf{A}$ & N/A & N/A & N/A \\
\hline $\mathrm{Ce}+3$ & $1.69 E+01$ & 3.53E-01 & $5.56 E+01$ & & $3.4 .2 E+01$ & $1.55 E+02$ & & \\
\hline Cl. & $3.61 E+01$ & $2.97 \mathrm{E}+01$ & $2.15 E+01$ & $1.75 E+01$ & $6.213 \mathrm{E}+00$ & $3.20 E+01$ & $4.13 \mathrm{E}-\mathrm{O} 2$ & 2.06E-02 \\
\hline $\mathrm{Co}+3$ & N/A & N/A & N/A & N/A & IV/A & N/A & N/A & N/A \\
\hline $\mathrm{CO}-2$ & $2.74 E+02$ & $2.18 \mathrm{E}+02$ & $2.83 \mathrm{E}+02$ & $1.14 E+02$ & $6.6 \mathrm{Y} E+01$ & $4.73 E+02$ & $2.17 E+01$ & $1.08 \mathrm{E}+07$ \\
\hline $\mathrm{Cr}+3$ & $9.48 \mathrm{E}+01$ & $2.19 E+01$ & $6.58 E+\infty$ & $7.22 \mathrm{E}+01$ & 2.0:2E +01 & $1.27 \mathrm{E}+01$ & 4.79E-O1 & 2.40E-01 \\
\hline Cs + & $4.77 \mathrm{E}-\mathrm{O} 2$ & 2.55E-05 & $3.07 \mathrm{E}-02$ & & $2.38 \mathrm{E}-02$ & $2.06 E-02$ & $1.03 E-05$ & \\
\hline $\mathrm{Cu}+2$ & $N / A$ & $\mathrm{~N} / \mathrm{A}$ & $\mathrm{N} / \mathrm{A}$ & $\mathrm{N} / \mathrm{A}$ & IN/A & N/A & N/A & N/A \\
\hline $\mathrm{F}$ & $5.38 \mathrm{E}+02$ & $3.95 E+01$ & $2.20 \mathrm{E}+01$ & 4.10E + 02 & $7.89 E+01$ & $3.29 E+01$ & & \\
\hline $\mathrm{Fe}+3$ & $7.38 E+03$ & $6.09 E+03$ & $2.29 E+03$ & $4.74 E+03$ & $1.39 \mathrm{E}+03$ & $3.24 \mathrm{E}+03$ & $3.24 \mathrm{E}+01$ & $1.62 E+01$ \\
\hline $\mathrm{Hg}+2$ & N/A & N/A & N/A & N/A & IN/A & N/A & $\mathrm{N} / \mathrm{A}$ & $\mathrm{N} / \mathrm{A}$ \\
\hline $\mathrm{K}+$ & & & $1.12 E+01$ & & & $4.78 \mathrm{E}+00$ & & \\
\hline \multicolumn{9}{|l|}{$\mathrm{La}+3$} \\
\hline $\mathrm{Mg}+2$ & N/A & N/A & $\mathrm{N} / \mathrm{A}$ & $\mathrm{N} / \mathrm{A}$ & IN/A & N/A & N/A & $N / A$ \\
\hline \multicolumn{9}{|l|}{$M n+4$} \\
\hline $\mathrm{Mo}+6$ & N/A & N/A & N/A & N/A & IN/A & N/A & N/A & N/A \\
\hline $\mathrm{Na}+$ & $9.31 \mathrm{E}+03$ & $2.11 E+03$ & $1.53 E+03$ & $6.50 \mathrm{E}+03$ & $1.59 E+03$ & $2.39 E+03$ & $6.56 \mathrm{E}+01$ & $3.28 \mathrm{E}+01$ \\
\hline $\mathrm{Ni}+3$ & $1.36 \mathrm{E}+03$ & $1.77 E+03$ & $8.51 E+03$ & $1.04 \mathrm{E}+03$ & $1.25 E+03$ & $1.33 E+04$ & $1.25 E+01$ & $6.24 \mathrm{E}+00$ \\
\hline NO2. & $5.87 E+01$ & $5.26 \mathrm{E}+00$ & $1.73 E+01$ & $4.43 E+01$ & $3.111 \mathrm{E}+01$ & $3.36 \mathrm{E}+01$ & & \\
\hline NO3- & $5.80 E+02$ & $5.88 \mathrm{E}+02$ & $6.05 E+02$ & $4.10 E+02$ & $1.96 \mathrm{GE}+02$ & $9.51 \mathrm{E}+02$ & $1.46 \mathrm{E}+00$ & $7.29 \mathrm{E}-01$ \\
\hline $\mathrm{OH}-$ & $2.39 \mathrm{E}+04$ & $9.58 E+03$ & $1.57 E+04$ & $2.26 \mathrm{E}+04$ & $2.3: 2 E+04$ & $3.79 \mathrm{E}+04$ & $2.06 \mathrm{E}+02$ & $1.03 E+02$ \\
\hline $\mathrm{Pb}+4$ & & & $1.75 E+04$ & & & $7.49 \mathrm{E}+03$ & & \\
\hline PO43 & $9.01 E+04$ & $7.81 E+03$ & $5.12 E+03$ & $6.86 E+04$ & 1.3 나E +04 & $7.74 \mathrm{E}+03$ & $2.47 \mathrm{E}+0.2$ & $1.24 E+02$ \\
\hline $\mathbf{R b}+$ & N/A & $N / A$ & N/A & N/A & IN/A & N/A & N/A & N/A \\
\hline $\mathrm{Re}+7$ & $\mathrm{~N} / \mathrm{A}$ & $\mathrm{N} / \mathrm{A}$ & N/A & N/A & N/A & $N / A$ & N/A & N/A \\
\hline $\mathrm{Rh}+\mathbf{3}$ & N/A & N/A & N/A & N/A & IJ/A & N/A & N/A & N/A \\
\hline$R u+3$ & N/A & N/A & N/A & N/A & IN/A & N/A & N/A & N/A \\
\hline $\mathrm{Se}+6$ & $N / A$ & N/A & N/A & N/A & IN/A & $N / A$ & N/A & N/A \\
\hline $5 i+4$ & & & & & & & $6.78 \mathrm{E}-02$ & $3.39 \mathrm{E}-02$ \\
\hline SO4-2 & $3.66 \mathrm{E}+01$ & $1.25 E+02$ & $4.68 \mathrm{E}+00$ & $2.22 E+01$ & $4.63 E+00$ & $5.54 E+O O$ & $5.03 E+00$ & $2.51 E+O 0$ \\
\hline $\mathrm{Sr}+2$ & 4.19E-01 & $1.12 \mathrm{E}-01$ & $3.44 \mathrm{E}-\mathrm{O} 4$ & $6.09 \mathrm{E}-02$ & 4.47E-01 & 1.87E-01 & $3.06 \mathrm{E}-05$ & \\
\hline Te +6 & $N / A$ & N/A & N/A & N/A & I I/A & N/A & N/A & $\mathrm{N} / \mathrm{A}$ \\
\hline$T h+4$ & N/A & N/A & N/A & N/A & IN/A & $N / A$ & N/A & N/A \\
\hline $\mathrm{TI}+4$ & N/A & N/A & N/A & N/A & $\mathbf{W} / \mathrm{A}$ & $\mathrm{N} / \mathrm{A}$ & N/A & $N / A$ \\
\hline TOC & $1.04 \mathrm{E}+03$ & $4.55 \mathrm{E}+01$ & $7.77 E+02$ & & 7.43E+01 & $1.12 E+03$ & & \\
\hline $\mathrm{UO} 2+2$ & $1.09 E+02$ & $7.39 \mathrm{E}+03$ & $1.29 E+04$ & $8.33 E+01$ & $3.08 \mathrm{E}+03$ & $2.34 E+04$ & $2.12 \mathrm{E}+\mathrm{O} 3$ & $1.06 \mathrm{E}+03$ \\
\hline$Z n+2$ & N/A & N/A & N/A & N/A & IN/A & $\mathrm{N} / \mathrm{A}$ & N/A & $\mathrm{N} / \mathrm{A}$ \\
\hline $\mathrm{ZrO} 2: 2 \mathrm{H} 2 \mathrm{O}$ & $7.80 E+03$ & $5.73 E+02$ & $3.18 \mathrm{E}+02$ & $5.94 \mathrm{E}+03$ & $1.14 \mathrm{LE}+03$ & $4.76 \mathrm{E}+02$ & & \\
\hline Total & $2.46 \mathrm{E}+05$ & $4.28 E+04$ & $7.08 E+04$ & $1.68 E+05$ & $6.63 E+04$ & $1.17 E+05$ & $2.72 \mathrm{E}+03$ & $1.36 \mathrm{E}+03$ \\
\hline
\end{tabular}


WHC-SD-WM-TI-784

Revision 0

Table A-1. Chemical Inventory Estimates for Single-Shell Tanks

\begin{tabular}{|c|c|c|c|c|c|c|c|c|}
\hline $\begin{array}{c}\text { Insoluble } \\
\text { (kg) }\end{array}$ & $C-107$ & C-108 & C- 109 & C-110 & C. 111 & $\mathrm{C}-112$ & C-201 & $\mathrm{C}-202$ \\
\hline \multicolumn{9}{|l|}{$\mathrm{Ag}+$} \\
\hline $\mathrm{Al}+3$ & $2.99 E+04$ & $2.48 E+03$ & $1.38 \mathrm{E}+03$ & $2.57 E+04$ & $1.4 \mid E+04$ & $1.25 E+04$ & & \\
\hline As +5 & N/A & N/A & $N / A$ & N/A & IN/A & N/A & N/A & N/A \\
\hline$B+3$ & $\mathrm{~N} / \mathrm{A}$ & N/A & N/A & $\mathrm{N} / \mathrm{A}$ & IS/A & N/A & N/A & N/A \\
\hline $\mathrm{Ba}+2$ & $2.31 E-01$ & $4.60 \mathrm{E}-01$ & 6.15E-01 & $7.57 \mathrm{E}-02$ & 5.84E-01 & $3.06 \mathrm{E}-01$ & $8.80 E-04$ & $1.08 \mathrm{E}-02$ \\
\hline $\mathrm{Be}+2$ & N/A & N/A & N/A & N/A & IN/A & N/A & $N / A$ & $\mathrm{~N} / \mathrm{A}$ \\
\hline$B i+3$ & $7.02 E+03$ & $5.15 E+02$ & $2.86 E+02$ & $5.35 E+03$ & 1.0.SE + 03 & $4.29 E+0.2$ & & \\
\hline $\mathrm{Ca}+2$ & $1.40 \mathrm{E}+0.3$ & $1.01 E+03$ & $2.09 E+03$ & $8.42 E+02$ & 4.9 4 E + 02 & $3.50 E+03$ & $9.75 E+00$ & $4.87 E+00$ \\
\hline Cancrinite & $6.50 E+04$ & $2.45 E+03$ & $1.36 E+03$ & $2.54 E+04$ & $5.1: E+03$ & $2.04 E+03$ & & \\
\hline $\mathrm{Cd}+2$ & N/A & N/A & $\mathrm{N} / \mathrm{A}$ & $\mathrm{N} / \mathrm{A}$ & IN/A & N/A & N/A & N/A \\
\hline $\mathrm{Ce}+3$ & $1.69 \mathrm{E}+01$ & $3.53 \mathrm{E}-01$ & $5.56 \mathrm{E}+01$ & & 3.4:E + 01 & $1.55 \mathrm{E}+02$ & & \\
\hline $\mathrm{Cl}-$ & $3.61 E+01$ & $2.97 E+01$ & $2.15 E+01$ & $1.75 \mathrm{E}+01$ & $6.28 \mathrm{E}+00$ & $3.20 \mathrm{E}+01$ & $4.13 \mathrm{E}-\mathrm{O} 2$ & 2.06E-02 \\
\hline $\mathrm{Co}+3$ & N/A & $\mathrm{N} / \mathrm{A}$ & N/A & $\mathrm{N} / \mathrm{A}$ & N/A & N/A & N/A & $\mathrm{N} / \mathrm{A}$ \\
\hline $\cos -2$ & $2.74 E+02$ & $2.18 E+02$ & $2.83 E+02$ & $1.14 E+02$ & $6.67 E+01$ & $4.73 E+02$ & $2.17 E+01$ & $1.08 \mathrm{E}+01$ \\
\hline $\mathrm{Cr}_{\mathrm{r}}+3$ & $9.48 E+01$ & $2.19 E+01$ & $6.58 \mathrm{E}+00$ & $7.22 \mathrm{E}+01$ & 2.02E+01 & $1.27 E+01$ & 4.79E-01 & $2.40 \mathrm{E}-01$ \\
\hline Cst & $4.77 \mathrm{E}-02$ & $2.55 \mathrm{E}-0.5$ & $3.07 E-02$ & & $2.38 \mathrm{BE}-02$ & $2.06 \mathrm{E}-02$ & $1.03 \mathrm{E}-05$ & \\
\hline $\mathrm{Cu}+2$ & N/A & N/A & $\mathrm{N} / \mathrm{A}$ & N/A & $\mathrm{N} / \mathrm{A}$ & $N / A$ & N/A & $\mathrm{N} / \mathrm{A}$ \\
\hline F- & $5.38 \mathrm{E}+\mathrm{O} 2$ & $3.95 \mathrm{E}+01$ & $2.20 \mathrm{E}+01$ & $4.10 \mathrm{E}+02$ & $7.8 \mathrm{~S}+\mathrm{E}+01$ & $3.29 \mathrm{E}+01$ & & \\
\hline $\mathrm{Fe}+3$ & $7.38 \mathrm{E}+03$ & $6.09 E+03$ & $2.29 E+03$ & $4.74 E+03$ & $1.3 \leqslant E+03$ & $3.24 \mathrm{E}+03$ & $3.24 \mathrm{E}+01$ & $1.62 \mathrm{E}+01$ \\
\hline $\mathrm{Hg}+2$ & $N / A$ & $N / A$ & N/A & N/A & $\mathrm{N} / \mathrm{A}$ & N/A & N/A & N/A \\
\hline$K+$ & & & $1.12 \mathrm{E}+01$ & & & $4.78 \mathrm{E}+00$ & & \\
\hline \multicolumn{9}{|l|}{$\mathrm{La}+3$} \\
\hline $\mathrm{Mg}+2$ & $\mathrm{~N} / \mathrm{A}$ & $N / A$ & N/A & N/A & N/A & $\mathrm{N} / \mathrm{A}$ & N/A & N/A \\
\hline \multicolumn{9}{|l|}{$\mathrm{Mn}+4$} \\
\hline $\mathrm{Mo}+6$ & N/A & $\mathrm{N} / \mathrm{A}$ & $N / A$ & N/A & $\mathbf{N} / \mathrm{A}$ & $\mathrm{N} / \mathrm{A}$ & N/A & N/A \\
\hline $\mathrm{Na}+$ & $9.31 \mathrm{E}+03$ & $2.11 E+03$ & $1.53 E+03$ & $6.50 E+03$ & $1.5 \mathrm{sE}+03$ & $2.39 E+03$ & $6.56 \mathrm{E}+01$ & $3.28 E+01$ \\
\hline $\mathrm{Ni}+3$ & $1.36 E+03$ & $1.77 E+03$ & $8.51 E+03$ & $1.04 E+03$ & $1.25 E+03$ & $1.33 E+04$ & $1.25 E+01$ & $6.24 \mathrm{E}+00$ \\
\hline $\mathrm{NO} 2-$ & $5.87 E+07$ & $5.26 \mathrm{E}+\infty$ & $1.73 \mathrm{E}+01$ & $4,43 E+01$ & $3.1 \varepsilon \mathrm{E}+01$ & $3.36 E+01$ & & \\
\hline NO3- & $5.80 E+02$ & $5.88 \mathrm{E}+02$ & $6.05 E+02$ & $4.10 \mathrm{E}+02$ & $1.96 E+02$ & $9.51 E+02$ & $1.46 \mathrm{E}+00$ & $7.29 \mathrm{E}-01$ \\
\hline $\mathrm{OH}-$ & $2.39 \mathrm{E}+04$ & $9.58 \mathrm{E}+03$ & $1.57 \mathrm{E}+04$ & $2.26 \mathrm{E}+04$ & $2.32 E+04$ & $3.79 E+04$ & $2.06 \mathrm{E}+02$ & $1.03 E+02$ \\
\hline $\mathrm{Pb}+4$ & & & $1.75 \mathrm{E}+04$ & & & $7.49 \mathrm{E}+03$ & & \\
\hline PO4-3 & $9.01 E+04$ & $7.81 E+03$ & $5.12 \mathrm{E}+03$ & $6.86 E+04$ & $1.34 E+04$ & $7.74 E+03$ & $2.47 \mathrm{E}+\mathrm{O} 2$ & $1.24 \mathrm{E}+02$ \\
\hline $\mathrm{Rb}+$ & N/A & N/A & $N / A$ & $N / A$ & N/A & N/A & $N / A$ & $N / A$ \\
\hline $\mathrm{Re}+7$ & N/A & N/A & $N / A$ & N/A & N/A & $\mathrm{N} / \mathrm{A}$ & N/A & N/A \\
\hline $\mathrm{Rh}+\mathbf{3}$ & N/A & N/A & $N / A$ & $N / A$ & N/A & $\mathrm{N} / \mathrm{A}$ & $N / A$ & $N / A$ \\
\hline$R u+3$ & N/A & N/A & N/A & $N / A$ & N/A & N/A & $\mathrm{N} / \mathrm{A}$ & N/A \\
\hline $\mathrm{Se}+6$ & N/A & N/A & $N / A$ & N/A & N/A & N/A & N/A & N/A \\
\hline$S i+4$ & & & & & & & $6.78 \mathrm{E}-\mathrm{O} 2$ & $3.39 \mathrm{E}-02$ \\
\hline SO4-2 & $3.66 \mathrm{E}+01$ & $1.25 E+02$ & $4.68 E+00$ & $2.22 E+01$ & $4.63 E+00$ & $5.54 \mathrm{E}+00$ & $5.03 E+00$ & $2.51 E+00$ \\
\hline $\mathrm{Sr}+2$ & 4.19E-01 & $1.12 \mathrm{E}-01$ & $3.44 E-04$ & $6.09 \mathrm{E}-02$ & 4.47E-01 & $1.87 \mathrm{E}-01$ & $3.06 \mathrm{E}-05$ & \\
\hline$T_{\theta}+6$ & N/A & N/A & N/A & N/A & $\mathrm{A} / \mathrm{A}$ & N/A & N/A & $N / A$ \\
\hline$T h+4$ & N/A & N/A & N/A & N/A & $\mathbf{N} / \mathbf{A}$ & $N / A$ & $\mathrm{~N} / \mathrm{A}$ & $\mathrm{N} / \mathrm{A}$ \\
\hline $\mathrm{TI}+4$ & N/A & N/A & $N / A$ & N/A & $\mathrm{N} / \mathrm{A}$ & N/A & N/A & N/A \\
\hline TOC & $1.04 E+03$ & $4.55 E+01$ & $7.77 \mathrm{E}+02$ & & $7.43 \mathrm{E}+01$ & $1.12 \mathrm{E}+03$ & & \\
\hline$\cup 02+2$ & $1.09 \mathrm{E}+02$ & $7.39 E+03$ & $1.29 \mathrm{E}+04$ & $8.33 E+01$ & $3.08 \mathrm{E}+03$ & $2.34 E+04$ & $2.12 \mathrm{E}+03$ & $1.06 \mathrm{E}+03$ \\
\hline$Z n+2$ & $\mathrm{~N} / \mathrm{A}$ & $N / A$ & $\mathrm{~N} / \mathrm{A}$ & $\mathrm{N} / \mathrm{A}$ & $\mathrm{A} / \mathrm{A}$ & N/A & $N / A$ & $N / A$ \\
\hline ZrO2:2H2O & $7.80 \mathrm{E}+03$ & $5.73 E+02$ & $3.18 \mathrm{E}+02$ & $5.94 \mathrm{E}+03$ & $1.14 \mathrm{E}+03$ & $4.76 \mathrm{E}+02$ & & \\
\hline Total & $2.46 \mathrm{E}+05$ & $4.28 E+04$ & $7.08 \mathrm{E}+04$ & $1.68 \mathrm{E}+05$ & $6.63 E+04$ & $1.17 \mathrm{E}+05$ & $2.72 \mathrm{E}+\mathrm{O} 3$ & $1.36 E+03$ \\
\hline
\end{tabular}


WHC-SD-WM-TI-784

Revision 0

Table A-1. Chemical Inventory Estimates for Single-Shell Tanks

\begin{tabular}{|c|c|c|c|c|c|c|c|c|}
\hline $\begin{array}{c}\text { Insoluble } \\
(\mathrm{kg})\end{array}$ & $C-203$ & C-204 & S-101 & S-102 & S-103 & s-104 & S-105 & S-106 \\
\hline \multicolumn{9}{|l|}{$\mathrm{Ag}+$} \\
\hline $\mathrm{Al}+3$ & & & $1.12 E+05$ & $1.99 E+03$ & $7.9 .4 E+01$ & $1.53 E+05$ & $1.16 E+03$ & $2.19 E+04$ \\
\hline $\mathrm{As}+5$ & N/A & N/A & $N / A$ & N/A & $\mathbf{N} / \mathbf{A}$ & N/A & $N / A$ & $N / A$ \\
\hline$B+3$ & N/A & N/A & N/A & N/A & N/A & N/A & $N / A$ & N/A \\
\hline$B a+2$ & $1.04 \mathrm{E}-02$ & & $3.16 \mathrm{E}+00$ & $3.05 E+00$ & $3.91 E+00$ & $2.68 E+00$ & $2.06 E+00$ & $2.10 \mathrm{E}+00$ \\
\hline $\mathrm{Be}+2$ & N/A & N/A & N/A & N/A & N/A & N/A & N/A & N/A \\
\hline$B i+3$ & & & $3.96 \mathrm{E}-01$ & 1.17E + OO & 4.EOE-O1 & & 7.04E-01 & $7.92 \mathrm{E}-01$ \\
\hline $\mathrm{Ca}+2$ & $2.44 E+01$ & $1.46 E+01$ & $1.35 E+03$ & $1.12 E+02$ & $1.42 E+01$ & $2.53 E+03$ & $5.28 E+01$ & $2.73 \mathrm{E}+02$ \\
\hline Cancrinite & & & $1.13 E+04$ & $1.60 E+04$ & $6.7 .2 E+03$ & $2.96 E+04$ & $1.31 E+04$ & $1.47 E+04$ \\
\hline $\mathrm{Cd}+2$ & N/A & N/A & N/A & N/A & IN/A & N/A & N/A & $N / A$ \\
\hline $\mathrm{Ce}+3$ & & & $5.88 \mathrm{E}+02$ & & & & & $1.48 \mathrm{E}-01$ \\
\hline $\mathrm{Cl}-$ & 1.03E-01 & $6.19 \mathrm{E}-02$ & $9.31 \mathrm{E}+01$ & $1.04 E+02$ & $4.113 E+01$ & $8.10 E+01$ & $7.26 E+01$ & $8.36 E+01$ \\
\hline $\mathrm{Co}+3$ & N/A & N/A & N/A & N/A & $\mathrm{IN} / \mathrm{A}$ & N/A & N/A & N/A \\
\hline $\mathrm{CO} \cdot 2$ & $5.42 \mathrm{E}+01$ & & $3.57 E+02$ & $4.16 \mathrm{E}+\mathrm{O} 2$ & $1.9: 2 \mathrm{E}+02$ & $3.41 E+02$ & $4.94 E+02$ & $5.87 \mathrm{E}+02$ \\
\hline $\mathrm{Cr}+3$ & $1.20 \mathrm{E}+00$ & $7.19 \mathrm{E}-01$ & $4.92 \mathrm{E}+03$ & $5.95 E+02$ & $8.40 \mathrm{E}+00$ & $1.14 E+04$ & $7.24 \mathrm{E}+01$ & $3.40 E+01$ \\
\hline $\mathrm{Cs}+$ & & & $5.32 \mathrm{E}-01$ & & & $1.89 \mathrm{E}-01$ & 5.21E-01 & $1.03 E+00$ \\
\hline $\mathrm{Cu}+2$ & N/A & N/A & N/A & N/A & IN/A & N/A & N/A & N/A \\
\hline $\mathrm{F}-$ & & & $1.84 E+01$ & $5.45 E+01$ & $2.1 .4 E+01$ & & $3.27 \mathrm{E}+01$ & $3.68 \mathrm{E}+01$ \\
\hline $\mathrm{Fe}+3$ & $8.11 E+01$ & $4.86 \mathrm{E}+01$ & $5.46 \mathrm{E}+03$ & $2.10 \mathrm{E}+02$ & $9.84 E-01$ & $1.11 \mathrm{E}+04$ & $5.51 E+01$ & $1.75 E+03$ \\
\hline $\mathrm{Hg}+2$ & N/A & N/A & N/A & N/A & IN/A & N/A & $\mathrm{N} / \mathrm{A}$ & N/A \\
\hline $\mathrm{K}+$ & & & $7.67 \mathrm{E}+00$ & $2.13 E+01$ & $8.75 E+00$ & & $1.58 \mathrm{E}+01$ & $1.78 \mathrm{E}+01$ \\
\hline \multicolumn{9}{|l|}{$\mathrm{La}+3$} \\
\hline $\mathrm{Mg}+2$ & N/A & N/A & N/A & N/A & N $/ A$ & N/A & N/A & N/A \\
\hline $\mathrm{Mn}+4$ & & & $9.95 E+02$ & $2.76 \mathrm{E}+03$ & $1.13 \mathrm{E}+03$ & & $2.05 E+03$ & $2.30 \mathrm{E}+03$ \\
\hline $\mathrm{Mo}+6$ & $N / A$ & N/A & N/A & $N / A$ & IN/A & N/A & N/A & N/A \\
\hline $\mathrm{Na}+$ & $1.64 E+02$ & $9.84 \mathrm{E}+01$ & $5.06 \mathrm{E}+03$ & $4.12 \mathrm{E}+03$ & 1.74E + 03 & $4.78 E+03$ & $3.75 E+03$ & $4.90 E+03$ \\
\hline $\mathrm{Ni}+3$ & $3.12 \mathrm{E}+01$ & $1.87 \mathrm{E}+01$ & $1.76 \mathrm{E}+03$ & $1.22 E+02$ & $5.45 E+00$ & $3.46 \mathrm{E}+03$ & $3.19 E+01$ & $3.79 \mathrm{E}+\mathrm{O} 2$ \\
\hline NO2- & & & $8.14 \mathrm{E}+02$ & $2.20 \mathrm{E}+03$ & & $6.88 \mathrm{E}+01$ & $1.38 E+03$ & $1.57 E+03$ \\
\hline NO3- & $3.65 E+00$ & & $8.02 \mathrm{E}+03$ & $1.39 E+04$ & $5.84 \mathrm{E}+03$ & $2.89 E+03$ & $1.55 E+04$ & $1.76 \mathrm{E}+04$ \\
\hline $\mathrm{OH}$ & $5.14 \mathrm{E}+02$ & & $2.24 \mathrm{E}+05$ & $2.74 \mathrm{E}+03$ & & $3.18 E+05$ & & $4.20 E+04$ \\
\hline $\mathrm{Pb}+4$ & & & $1.15 \mathrm{E}-03$ & $3.20 \mathrm{E}-03$ & $1.31 \mathrm{E}-03$ & & $2.37 \mathrm{E}-03$ & $2.67 \mathrm{E}-03$ \\
\hline $\mathrm{PO} 4-3$ & $6.18 \mathrm{E}+02$ & $3.71 E+02$ & $7.13 \mathrm{E}+01$ & $2.08 E+02$ & $8.24 \mathrm{E}+01$ & & $1.32 E+02$ & $1.49 \mathrm{E}+02$ \\
\hline $\mathrm{Rb}+$ & N/A & $N / A$ & $N / A$ & $N / A$ & IN/A & N/A & N/A & $\mathrm{N} / \mathrm{A}$ \\
\hline$R \theta+7$ & $N / A$ & $N / A$ & N/A & N/A & N/A & N/A & N/A & N/A \\
\hline$R h+3$ & $\mathrm{~N} / \mathrm{A}$ & N/A & $N / A$ & N/A & IN/A & N/A & N/A & $\mathrm{N} / \mathrm{A}$ \\
\hline $\mathrm{Ru}+\mathbf{3}$ & $N / A$ & $N / A$ & $N / A$ & N/A & IN/A & N/A & N/A & $\mathrm{N} / \mathrm{A}$ \\
\hline Se+6 & $N / A$ & N/A & N/A & N/A & IN/A & N/A & N/A & $N / A$ \\
\hline $\mathrm{Si}+4$ & $1.70 \mathrm{E}-01$ & $1.02 \mathrm{E}-01$ & & & & & & \\
\hline SO4-2 & $1.26 E+01$ & $7.54 \mathrm{E}+00$ & $7.38 \mathrm{E}+01$ & $1.70 \mathrm{E}+02$ & 7.3:E +01 & $1.15 \mathrm{E}+01$ & $1.54 \mathrm{E}+\mathrm{O} 2$ & $1.73 E+02$ \\
\hline $\mathrm{Sr}+2$ & 3.2OE-03 & & $2.52 \mathrm{E}+01$ & 2.67E-01 & & $4.50 E+01$ & $2.40 \mathrm{E}-01$ & $2.60 E+01$ \\
\hline Te+6 & N/A & N/A & N/A & N/A & N/A & N/A & N/A & $N / A$ \\
\hline$T h+4$ & N/A & $N / A$ & N/A & N/A & N/A & $N / A$ & $\mathrm{~N} / \mathrm{A}$ & N/A \\
\hline$T+4$ & $\mathrm{~N} / \mathrm{A}$ & N/A & N/A & N/A & N/A & N/A & N/A & N/A \\
\hline TOC & & & 4.47E +01 & $1.40 E+02$ & 5.4EE + 01 & & $8.17 E+01$ & $9.16 \mathrm{E}+01$ \\
\hline $\mathrm{U} 02+2$ & $5.29 E+03$ & $2.80 E+00$ & $7.43 E+03$ & $7.54 E+02$ & $6.24 \mathrm{E}-01$ & $2.08 \mathrm{E}+04$ & $2.80 E+01$ & $7.56 \mathrm{E}+03$ \\
\hline$Z n+2$ & N/A & N/A & N/A & N/A & N/A & N/A & N/A & N/A \\
\hline \multicolumn{9}{|l|}{$\mathrm{ZrO} 2: 2 \mathrm{H} 2 \mathrm{O}$} \\
\hline Total & $6.80 \mathrm{E}+03$ & $5.62 \mathrm{E}+02$ & $3.84 E+05$ & $4.67 E+04$ & $1.60 \mathrm{E}+04$ & $5.59 E+05$ & $3.82 \mathrm{E}+04$ & $1.16 \mathrm{E}+05$ \\
\hline
\end{tabular}


WHC-SD-WM-TI-784

Revision 0

Table A-1. Chemical Inventory Estimates for Single-Shell Tanks

\begin{tabular}{|c|c|c|c|c|c|c|c|c|}
\hline $\begin{array}{c}\text { Insoluble } \\
\text { (kg) }\end{array}$ & S-107 & $5-108$ & S- 109 & S.110 & s.111 & S-112 & SX-101 & SX-102 \\
\hline $\mathrm{Ag}+$ & & & & & & 2.34E-05 & & \\
\hline$A \mathbf{I}+\mathbf{3}$ & $9.90 E+04$ & $2.46 E+03$ & $9.05 E+03$ & $5.72 \mathrm{E}+04$ & $4.1 \mathrm{IE}+04$ & $3.49 E+03$ & $1.69 E+05$ & $2.87 E+04$ \\
\hline$A s+5$ & N/A & $\mathrm{N} / \mathrm{A}$ & N/A & N/A & $I V / A$ & N/A & N/A & N/A \\
\hline$B+3$ & N/A & $N / A$ & $N / A$ & N/A & $\mathrm{N} / \mathrm{A}$ & N/A & N/A & N/A \\
\hline $\mathrm{Ba}+2$ & $1.11 E+00$ & $2.01 E+00$ & $5.03 E+00$ & $4.63 E+00$ & 4.5:3E+O0 & $1.20 E+01$ & $4.22 E+00$ & $3.72 \mathrm{E}+\infty$ \\
\hline $\mathrm{Be}+2$ & N/A & $\mathrm{N} / \mathrm{A}$ & $\mathrm{N} / \mathrm{A}$ & $\mathrm{N} / \mathrm{A}$ & $\mathrm{IN} / \mathrm{A}$ & N/A & $N / A$ & $\mathrm{~N} / \mathrm{A}$ \\
\hline$B i+3$ & 2.07E-01 & $9.28 \mathrm{E}-01$ & $8.60 \mathrm{E}-01$ & $4.29 \mathrm{E}-01$ & $6.92 \mathrm{E}-01$ & $9.77 \mathrm{E}-01$ & $2.41 \mathrm{E}-01$ & $7.95 \mathrm{E}-01$ \\
\hline $\mathrm{Ca}+2$ & $1.35 E+03$ & $1.58 \mathrm{E}+02$ & $1.38 E+02$ & $7.38 E+02$ & $6.613 \mathrm{E}+02$ & $1.66 \mathrm{E}+02$ & $2.11 E+03$ & $4.62 E+02$ \\
\hline Cancrinite & $1.19 E+04$ & $1.73 E+04$ & $1.60 E+04$ & $9.21 E+03$ & $1.5 \mathrm{IE}+04$ & $1.82 E+04$ & $7.26 \mathrm{E}+03$ & $4.37 E+04$ \\
\hline $\mathrm{Cd}+2$ & N/A & N/A & N/A & N/A & IN/A & $\mathrm{N} / \mathrm{A}$ & $\mathrm{N} / \mathrm{A}$ & $N / A$ \\
\hline $\mathrm{C}_{\mathrm{B}}+3$ & & $7.20 E+02$ & $1.00 \mathrm{E}+03$ & $9.85 \mathrm{E}+01$ & $1.013 \mathrm{E}+02$ & $6.81 \mathrm{E}+03$ & & $2.67 E+00$ \\
\hline Cl- & $7.44 \mathrm{E}+01$ & $9.71 E+01$ & $8.90 E+01$ & $7.73 E+01$ & $9.113 E+01$ & $1.02 E+02$ & $1.20 E+02$ & $9.76 \mathrm{E}+01$ \\
\hline $\mathrm{Co}+3$ & N/A & $\mathrm{N} / \mathrm{A}$ & N/A & N/A & IV/A & N/A & N/A & N/A \\
\hline $\mathrm{CO}-2$ & $2.60 E+02$ & $6.63 E+02$ & $6.15 E+02$ & $3.96 \mathrm{E}+02$ & $5.70 \mathrm{E}+02$ & $6.98 \mathrm{E}+02$ & $3.88 \mathrm{E}+02$ & $5.58 \mathrm{E}+02$ \\
\hline $\mathrm{Cr}+3$ & $4.77 E+03$ & $7.64 E+02$ & $2.86 \mathrm{E}+01$ & $3.00 E+03$ & $1.99 \mathrm{E}+03$ & $5.31 \mathrm{E}+02$ & $8.80 E+03$ & $4.59 E+03$ \\
\hline $\mathrm{Cs}+$ & & $1.39 E+00$ & $7.82 E+00$ & $8.73 E+00$ & 8.01:5E + 00 & $2.71 E+01$ & & $2.41 \mathrm{E}-03$ \\
\hline $\mathrm{Cu}+2$ & N/A & $\mathrm{N} / \mathrm{A}$ & N/A & N/A & IN/A & N/A & N/A & $\mathrm{N} / \mathrm{A}$ \\
\hline F- & $1.62 \mathrm{E}+03$ & $4.31 E+01$ & $3.99 \mathrm{E}+01$ & $1.99 E+01$ & 3.2 $E+01$ & $4.54 E+01$ & $1.12 \mathrm{E}+01$ & $3.69 E+01$ \\
\hline $\mathrm{Fe}+3$ & $6.52 E+03$ & $3.03 E+02$ & $5.86 \mathrm{E}+02$ & $2.95 E+03$ & $2.018 E+03$ & $3.90 \mathrm{E}+\mathrm{O} 2$ & $8.68 E+03$ & $3.98 E+03$ \\
\hline $\mathrm{Hg}+2$ & N/A & N/A & N/A & $\mathrm{N} / \mathrm{A}$ & IN/A & N/A & N/A & N/A \\
\hline $\mathrm{K}+$ & $5.18 \mathrm{E}+00$ & $2.08 \mathrm{E}+01$ & $1.93 E+01$ & $9.63 \mathrm{E}+00$ & $1.55 \mathrm{E}+01$ & $2.19 \mathrm{E}+01$ & $4.64 E+00$ & $1.72 E+01$ \\
\hline \multicolumn{9}{|l|}{$\mathrm{La}+3$} \\
\hline $\mathrm{Mg}+2$ & N/A & N/A & N/A & N/A & IN/A & $N / A$ & N/A & $N / A$ \\
\hline$M n+4$ & $3.31 \mathrm{E}+02$ & $2.70 \mathrm{E}+03$ & $2.50 \mathrm{E}+03$ & $1.25 E+03$ & $2.0^{\circ} \mathrm{E}+03$ & $2.84 \mathrm{E}+03$ & $6.01 \mathrm{E}+02$ & $2.23 E+03$ \\
\hline $\mathrm{Mo}+6$ & $N / A$ & $\mathrm{~N} / \mathrm{A}$ & N/A & N/A & N/A & $\mathrm{N} / \mathrm{A}$ & N/A & $\mathrm{N} / \mathrm{A}$ \\
\hline $\mathrm{Na}+$ & $5.09 E+03$ & $5.05 E+03$ & $4.84 E+03$ & $4.03 E+03$ & $4.8 B E+03$ & $5.28 E+03$ & $6.12 \mathrm{E}+03$ & $4.85 E+03$ \\
\hline $\mathrm{Ni}+3$ & $1.98 E+03$ & $1.63 E+02$ & $1.49 E+02$ & $9.39 E+02$ & $8.18 \mathrm{E}+02$ & $1.79 E+02$ & $2.74 E+03$ & $6.84 E+02$ \\
\hline NO2- & $4.22 E+02$ & $1.82 E+03$ & $1.69 E+03$ & $8.41 E+02$ & $1.38 \mathrm{E}+03$ & $1.92 \mathrm{E}+03$ & $4.57 E+02$ & $1.54 \varepsilon+03$ \\
\hline $\mathrm{NO3}-$ & $8.08 E+03$ & $2.05 E+04$ & $1.90 E+04$ & $1.07 \mathrm{E}+04$ & $1.60 \mathrm{E}+04$ & $2.16 \mathrm{E}+04$ & $7.24 E+03$ & $1.65 E+04$ \\
\hline $\mathrm{OH}$ & $2.00 E+05$ & $3.10 \mathrm{E}+03$ & $1.58 E+04$ & $1.14 \mathrm{E}+05$ & $8.10 E+04$ & $7.00 E+03$ & $3.41 E+05$ & $6.13 E+04$ \\
\hline $\mathrm{Pb}+4$ & $3.84 \mathrm{E}-04$ & $3.13 \mathrm{E}-03$ & $2.90 \mathrm{E}-03$ & $1.45 \mathrm{E}-03$ & $2.33 \mathrm{E}-03$ & 3.30E-03 & 6.97E-04 & $2.58 \mathrm{E}-03$ \\
\hline PO4-3 & $3.37 \mathrm{E}+01$ & $1.74 \mathrm{E}+\mathrm{O} 2$ & $1.61 E+02$ & $8.05 E+01$ & $1.30 E+02$ & $1.83 E+02$ & $4.33 \mathrm{E}+01$ & $1.47 E+02$ \\
\hline $\mathbf{R b}+$ & $N / A$ & N/A & N/A & N/A & IN/A & $N / A$ & N/A & N/A \\
\hline$R e+7$ & N/A & $\mathrm{N} / \mathrm{A}$ & N/A & N/A & $\mathrm{N} / \mathrm{A}$ & $N / A$ & $N / A$ & $\mathrm{~N} / \mathrm{A}$ \\
\hline$R h+3$ & $\mathrm{~N} / \mathrm{A}$ & N/A & $\mathrm{N} / \mathrm{A}$ & N/A & $\mathrm{N} / \mathrm{A}$ & N/A & N/A & N/A \\
\hline$R u+3$ & $N / A$ & N/A & $\mathrm{N} / \mathrm{A}$ & $\mathrm{N} / \mathrm{A}$ & $\mathrm{N} / \mathrm{A}$ & $\mathrm{N} / \mathrm{A}$ & N/A & $\mathrm{N} / \mathrm{A}$ \\
\hline $5 e+6$ & $N / A$ & N/A & $\mathrm{N} / \mathrm{A}$ & $\mathrm{N} / \mathrm{A}$ & $\mathrm{N} / \mathrm{A}$ & N/A & N/A & N/A \\
\hline \multicolumn{9}{|l|}{$\mathrm{Si}+4$} \\
\hline 504-2 & $5.50 E+01$ & $2.02 \mathrm{E}+02$ & $1.87 E+02$ & $9.84 E+01$ & $1.54 \mathrm{E}+02$ & $2.13 E+02$ & $5.40 E+01$ & $1.64 \mathrm{E}+02$ \\
\hline $\mathrm{s} r+2$ & $1.36 \mathrm{E}+01$ & $3.71 \mathrm{E}-01$ & $1.10 E+01$ & $2.89 \mathrm{E}+00$ & $1.3 \leqslant E+01$ & $9.58 \mathrm{E}+\infty 0$ & $3.64 E+00$ & $1.03 E+01$ \\
\hline Te + 6 & $N / A$ & N/A & N/A & $N / A$ & $N / A$ & $N / A$ & $N / A$ & N/A \\
\hline Th +4 & $N / A$ & N/A & N/A & $\mathrm{N} / \mathrm{A}$ & $\mathrm{N} / \mathrm{A}$ & $\mathrm{N} / \mathrm{A}$ & $N / A$ & N/A \\
\hline $\mathrm{Tl}+4$ & N/A & N/A & N/A & $N / A$ & $\mathrm{~N} / \mathrm{A}$ & N/A & $N / A$ & $\mathrm{~N} / \mathrm{A}$ \\
\hline TOC & $1.75 \mathrm{E}+01$ & $1.08 E+02$ & $9.97 \mathrm{E}+01$ & $4.78 \mathrm{E}+01$ & $7.85 \mathrm{E}+01$ & $1.13 E+02$ & $2.63 E+01$ & $9.40 E+01$ \\
\hline $\mathrm{UO2}+2$ & $4.51 E+03$ & $9.03 E+02$ & $2.85 \mathrm{E}+\mathrm{O} 3$ & $1.51 E+03$ & $3.74 E+03$ & $1.30 E+03$ & $4.45 E+03$ & $2.24 \mathrm{E}+03$ \\
\hline$Z n+2$ & N/A & N/A & N/A & N/A & II/A & N/A & N/A & N/A \\
\hline $\mathrm{ZrO} 2: 2 \mathrm{H} 2 \mathrm{O}$ & $2.33 E+05$ & & & & & & & \\
\hline Total & $5.79 \mathrm{E}+05$ & $5.72 \mathrm{E}+04$ & $7.48 E+04$ & $2.07 E+05$ & $1.7: E+05$ & $7.11 \mathrm{E}+04$ & $5.59 E+05$ & $1.72 \mathrm{E}+05$ \\
\hline
\end{tabular}


WHC-SD-WM-TI-784

Revision 0

Table A-1. Chemical Inventory Estimates for Single-Shell Tanks

\begin{tabular}{|c|c|c|c|c|c|c|c|c|}
\hline $\begin{array}{c}\text { Insoluble } \\
(\mathrm{kg})\end{array}$ & sx-103 & sx-104 & sx-105 & sX-106 & $5 x-107$ & $5 x-108$ & sx-109 & $5 x-110$ \\
\hline $\mathrm{Ag}+$ & $1.14 \mathrm{E}-05$ & & & & & & & \\
\hline$A l+3$ & $5.67 E+04$ & $8.55 E+04$ & $2.70 E+04$ & $6.16 \mathrm{E}+02$ & 4.8 $5 \mathrm{E}+04$ & $5.41 E+04$ & $3.70 E+04$ & $8.70 E+03$ \\
\hline$A s+5$ & N/A & N/A & N/A & N/A & N $/ \mathbf{A}$ & N/A & $N / A$ & $N / A$ \\
\hline$B+3$ & N/A & N/A & N/A & $\mathrm{N} / \mathrm{A}$ & IV/A & N/A & N/A & N/A \\
\hline $\mathrm{Ba}+2$ & $1.90 E+01$ & $4.33 \mathrm{E}+00$ & $3.71 E+00$ & $2.35 E-01$ & $6.13 \mathrm{E}+00$ & $6.04 \mathrm{E}+00$ & $4.82 \mathrm{E}+\infty 0$ & $1.32 E+01$ \\
\hline $\mathrm{Be}+2$ & N/A & N/A & N/A & N/A & IN/A & N/A & $N / A$ & $N / A$ \\
\hline$B i+3$ & $8.36 \mathrm{E}-01$ & $6.89 \mathrm{E}-01$ & $9.67 \mathrm{E}-01$ & $1.11 E+00$ & & & & \\
\hline $\mathrm{Ca}+2$ & $7.56 \mathrm{E}+02$ & $1.11 E+03$ & $5.85 \mathrm{E}+02$ & $4.05 E+01$ & $1.06 E+03$ & $1.13 E+03$ & $1.31 E+03$ & $9.48 E+02$ \\
\hline Cancrinite & $1.67 \mathrm{E}+04$ & $1.46 E+04$ & $3.04 E+04$ & $1.40 E+04$ & $2.50 E+04$ & $2.51 E+04$ & $4.59 \mathrm{E}+04$ & $3.49 \mathrm{E}+04$ \\
\hline $\mathrm{Cd}+2$ & N/A & N/A & N/A & N/A & IN/A & $N / A$ & $\mathrm{~N} / \mathrm{A}$ & N/A \\
\hline $\mathrm{C}_{\theta+3}$ & $4.63 E+03$ & $9.55 E+02$ & $1.58 E+03$ & $2.93 E+03$ & & & & \\
\hline $\mathrm{Cl}$ & $1.19 E+02$ & $1.20 \mathrm{E}+\mathrm{O} 2$ & $1.17 E+02$ & $9.42 \mathrm{E}+01$ & $3.5: 2 \mathrm{E}+01$ & $3.85 \mathrm{E}+01$ & $1.85 \mathrm{E}+01$ & $1.02 \mathrm{E}+01$ \\
\hline $\mathrm{Co}+3$ & N/A & N/A & N/A & $N / A$ & IN/A & $\mathrm{N} / \mathrm{A}$ & N/A & N/A \\
\hline $\mathrm{CO}-2$ & $6.80 \mathrm{E}+02$ & $6.26 \mathrm{E}+\mathrm{O} 2$ & $7.37 E+02$ & $3.06 \mathrm{E}+02$ & $1.43 E+02$ & $1.53 E+02$ & $1.56 E+02$ & $1.16 E+02$ \\
\hline $\mathrm{Cr}+3$ & $2.99 \mathrm{E}+03$ & $4.49 \mathrm{E}+03$ & $3.70 \mathrm{E}+03$ & $1.97 E+02$ & $7.72 \mathrm{E}+03$ & $8.01 E+03$ & $8.59 E+03$ & $7.41 \mathrm{E}+03$ \\
\hline $\mathrm{Cs}+$ & $1.01 E+01$ & $6.91 \mathrm{E}-01$ & $1.43 E+O O$ & $1.39 \mathrm{E}+00$ & & & & \\
\hline $\mathrm{Cu}+2$ & N/A & N/A & N/A & N/A & IV/A & $\mathrm{N} / \mathrm{A}$ & N/A & N/A \\
\hline$F_{-}$ & $3.88 \mathrm{E}+01$ & $3.20 \mathrm{E}+01$ & $4.49 \mathrm{E}+01$ & $5.16 \mathrm{E}+01$ & & & & \\
\hline $\mathrm{Fe}+3$ & $2.93 \mathrm{E}+0.3$ & $4.41 \mathrm{E}+03$ & $2.49 \mathrm{E}+03$ & $7.40 E+01$ & $5.06 \mathrm{E}+03$ & $5.35 E+03$ & $4.27 E+03$ & $3.64 \mathrm{E}+03$ \\
\hline $\mathrm{Hg}+2$ & N/A & $N / A$ & N/A & N/A & N/A & N/A & N/A & N/A \\
\hline $\mathrm{K}+$ & $1.88 \mathrm{E}+01$ & $1.55 E+01$ & $2.12 \mathrm{E}+01$ & $1.92 \mathrm{E}+01$ & & & & \\
\hline \multicolumn{9}{|l|}{$\mathrm{La}+3$} \\
\hline $\mathrm{Mg}+2$ & $N / A$ & N/A & N/A & N/A & IN/A & N/A & $\mathrm{N} / \mathrm{A}$ & N/A \\
\hline $\mathrm{Mn}+4$ & $2.43 E+03$ & $2.00 E+03$ & $2.75 \mathrm{E}+03$ & $2.49 \mathrm{E}+03$ & & & & \\
\hline $\mathrm{Mo}+6$ & $N / A$ & N/A & N/A & N/A & IN/A & N/A & N/A & $N / A$ \\
\hline $\mathrm{Na}+$ & $6.16 \mathrm{E}+03$ & $6.27 \mathrm{E}+03$ & $6.19 \mathrm{E}+03$ & $3.39 \mathrm{E}+03$ & $1.80 E+03$ & $1.97 \mathrm{E}+\mathrm{O} 3$ & $2.42 E+O 3$ & $1.76 \mathrm{E}+03$ \\
\hline $\mathrm{Ni}+3$ & $9.41 E+0.2$ & $1.40 \mathrm{E}+03$ & $7.39 \mathrm{E}+02$ & $3.66 \mathrm{E}+01$ & $1.45 \mathrm{E}+03$ & $1.54 E+03$ & $1.66 E+03$ & $1.10 E+03$ \\
\hline No2- & $1.64 \mathrm{E}+03$ & $1.35 \mathrm{E}+03$ & $1.89 \mathrm{E}+03$ & $2.07 E+03$ & & & $5.68 E+01$ & $1.17 E+01$ \\
\hline NO3- & $1.97 E+04$ & $1.71 \mathrm{E}+04$ & $2.24 \mathrm{E}+04$ & $1.09 E+04$ & $1.1 E+03$ & $1.23 E+03$ & $1.84 E+04$ & $3.84 E+03$ \\
\hline $\mathrm{OH}-$ & $1.14 \mathrm{E}+05$ & $1.71 \mathrm{E}+05$ & $5.58 \mathrm{E}+04$ & $9.87 \mathrm{E}+02$ & $1.0 \% \mathrm{E}+05$ & $1.18 \mathrm{E}+05$ & $8.12 E+04$ & $2.92 \mathrm{E}+04$ \\
\hline $\mathrm{Pb}+4$ & $2.82 \mathrm{E}-03$ & $2.32 \mathrm{E}-03$ & $3.19 \mathrm{E}-03$ & 2.89E-03 & & & & \\
\hline PO4-3 & $1.57 E+02$ & $1.29 \mathrm{E}+02$ & $1.80 E+02$ & $1.94 E+02$ & & & & \\
\hline $\mathbf{R b}+$ & $N / A$ & N/A & $\mathrm{N} / \mathrm{A}$ & $N / A$ & N/A & N/A & N/A & N/A \\
\hline $\mathrm{Re}+7$ & N/A & N/A & N/A & $N / A$ & N/A & N/A & N/A & N/A \\
\hline $\mathrm{Rh}+3$ & $N / A$ & $N / A$ & $N / A$ & $N / A$ & IN/A & N/A & N/A & N/A \\
\hline $\mathrm{Ru}+3$ & N/A & N/A & N/A & N/A & IN/A & N/A & N/A & N/A \\
\hline Se+6 & $N / A$ & $N / A$ & $N / A$ & $N / A$ & N/A & N/A & N/A & N/A \\
\hline \multicolumn{9}{|l|}{$S i+4$} \\
\hline SO4-2 & $1.87 \mathrm{E}+02$ & $1.57 \mathrm{E}+02$ & $2.15 E+O 2$ & $1.44 E+02$ & $4.8 \% E+\infty$ & $5.35 \mathrm{E}+00$ & $3.46 E+0 O$ & $1.25 E+00$ \\
\hline$S r+2$ & $3.54 E+00$ & $3.64 \mathrm{E}-01$ & $1.86 \mathrm{E}+00$ & $8.16 \mathrm{E}-01$ & 9.0\%E+OO & $5.64 E+00$ & $1.70 E+O O$ & $3.71 E+00$ \\
\hline$T_{\theta}+6$ & N/A & N/A & N/A & N/A & N/A & N/A & N/A & N/A \\
\hline $\mathrm{Th}+4$ & N/A & N/A & N/A & N/A & IN/A & $N / A$ & $N / A$ & $\mathrm{~N} / \mathrm{A}$ \\
\hline$T i+4$ & N/A & N/A & N/A & N/A & N/A & $N / A$ & N/A & N/A \\
\hline TOC & $9.47 E+01$ & $7.69 E+01$ & $1.10 E+02$ & $1.33 E+02$ & & & & \\
\hline $\mathrm{uO2}+2$ & $1.50 E+03$ & $2.26 \mathrm{E}+03$ & $3.35 \mathrm{E}+03$ & $2.18 E+02$ & $7.25 \mathrm{E}+03$ & $7.40 \mathrm{E}+03$ & $7.25 E+03$ & $8.41 E+03$ \\
\hline$Z n+2$ & N/A & N/A & N/A & $\mathrm{N} / \mathrm{A}$ & N/A & $N / A$ & N/A & N/A \\
\hline \multicolumn{9}{|l|}{$\mathrm{ZrO} 2: 2 \mathrm{H} 2 \mathrm{O}$} \\
\hline Total & $2.32 \mathrm{E}+05$ & $3.13 E+05$ & $1.60 E+05$ & $3.89 E+04$ & $2.06 \mathrm{E}+05$ & $2.24 \mathrm{E}+05$ & $2.08 E+05$ & $1.00 E+05$ \\
\hline
\end{tabular}


WHC-SD-WM-TI-784

Revision 0

Table A-1. Chemical Inventory Estimates for Single-Shell Tanks

\begin{tabular}{|c|c|c|c|c|c|c|c|c|}
\hline $\begin{array}{c}\text { Insoluble } \\
\text { (kg) }\end{array}$ & $s \times-111$ & $s x-112$ & $5 x-113$ & sx-114 & $s \times-115$ & $\mathrm{~T}-101$ & $\mathrm{~T}-102$ & $T-103$ \\
\hline \multicolumn{9}{|l|}{$\mathrm{AQ}+$} \\
\hline$A \mathrm{I}+3$ & $3.19 E+04$ & $3.10 E+04$ & $1.02 E+03$ & $1.02 E+04$ & $1.65 E+03$ & $2.28 E+04$ & $1.10 \mathrm{E}+04$ & $1.10 E+04$ \\
\hline $\mathrm{As}+5$ & $\mathrm{~N} / \mathrm{A}$ & N/A & $N / A$ & N/A & $N / A$ & N/A & N/A & $\mathrm{N} / \mathrm{A}$ \\
\hline$B+3$ & N/A & N/A & $N / A$ & $N / A$ & $N / A$ & N/A & N/A & $\mathrm{N} / \mathrm{A}$ \\
\hline $\mathrm{Ba}+2$ & $9.34 \mathrm{E}+00$ & $4.28 \mathrm{E}+00$ & $4.34 \mathrm{E}+00$ & $1.16 \mathrm{E}+01$ & $8.25 \mid \equiv+\infty$ & $4.50 \mathrm{E}-01$ & $2.36 \mathrm{E}-03$ & 9.27E-O2 \\
\hline $\mathrm{Be}+2$ & N/A & N/A & N/A & N/A & N/A & N/A & N/A & $\mathrm{N} / \mathrm{A}$ \\
\hline$B i+3$ & & & & & & & & 5.79E-01 \\
\hline $\mathrm{Ca}+2$ & $1.53 E+03$ & $1.04 \mathrm{E}+03$ & $1.27 E+01$ & $1.51 E+03$ & $9.89 E+01$ & $5.68 E+02$ & $2.78 E+02$ & $2.75 \mathrm{E}+02$ \\
\hline Cancrinite & $4.57 E+04$ & $2.69 E+04$ & $2.22 \mathrm{E}+01$ & $6.11 E+04$ & $6.62 \mathrm{I}+03$ & $3.72 E+00$ & $3.7 \mathrm{OE}+00$ & $1.32 \mathrm{E}+02$ \\
\hline $\mathrm{Cd}+2$ & N/A & N/A & N/A & N/A & $\mathbf{N} / \mathbf{A}$ & N/A & $N / A$ & N/A \\
\hline $\mathrm{Ce}+3$ & & & & & & $7.94 E+02$ & $1.35 E+01$ & 2.74E-01 \\
\hline $\mathrm{Cl} \cdot$ & $2.35 E+01$ & $2.09 E+01$ & 5.93E-01 & $1.38 \mathrm{E}+01$ & $1.611 E+\infty$ & $3.81 E+00$ & $1.38 \mathrm{E}+00$ & $2.06 \mathrm{E}+00$ \\
\hline $\mathrm{C}_{0}+3$ & N/A & $\mathrm{N} / \mathrm{A}$ & N/A & N/A & $N / A$ & N/A & N/A & N/A \\
\hline $\mathrm{CO}-2$ & $1.90 E+02$ & $1.31 E+02$ & $1.71 E+\infty 0$ & $1.90 E+02$ & $1.25 \equiv+01$ & $9.71 E+01$ & $5.78 E+01$ & $4.84 E+01$ \\
\hline $\mathrm{Cr}+3$ & $1.07 \mathrm{E}+04$ & $6.97 E+03$ & $5.31 \mathrm{E}+01$ & $1.09 E+04$ & $1.15 \equiv+03$ & $1.31 E+01$ & $6.55 \mathrm{E}+00$ & $6.77 E+00$ \\
\hline $\mathrm{Cs}+$ & & $1.68 \mathrm{E}-02$ & $2.52 \mathrm{E}+00$ & & $5.10 \equiv+\infty 0$ & $1.51 E-01$ & $2.56 \mathrm{E}-03$ & $1.23 \mathrm{E}-02$ \\
\hline $\mathrm{Cu}+2$ & N/A & N/A & N/A & $N / A$ & NIA & N/A & $N / A$ & N/A \\
\hline F- & & & & & & & & $1.36 \mathrm{E}+00$ \\
\hline $\mathrm{Fe}+3$ & $5.79 E+03$ & $4.10 E+03$ & $5.24 E+01$ & $4.59 E+03$ & $6.671 \equiv+02$ & $2.37 E+03$ & $1.16 \mathrm{E}+03$ & $1.15 \mathrm{E}+03$ \\
\hline $\mathrm{Hg}+2$ & $\mathrm{~N} / \mathrm{A}$ & N/A & N/A & N/A & $N / A$ & N/A & N/A & $\mathrm{N} / \mathrm{A}$ \\
\hline \multicolumn{9}{|l|}{$\mathrm{K}+$} \\
\hline \multicolumn{9}{|l|}{$\operatorname{La}+3$} \\
\hline $\mathrm{Mg}+2$ & $\mathrm{~N} / \mathrm{A}$ & N/A & N/A & N/A & N/A & N/A & $N / A$ & N/A \\
\hline \multicolumn{9}{|l|}{$\mathrm{Mn}+4$} \\
\hline $\mathrm{Mo}+6$ & N/A & N/A & $N / A$ & N/A & NIA & N/A & $N / A$ & $\mathrm{~N} / \mathrm{A}$ \\
\hline $\mathrm{Ne}+$ & $3.14 \varepsilon+03$ & $2.16 \mathrm{E}+03$ & $3.12 \mathrm{E}+01$ & $3.93 \mathrm{E}+03$ & $2.39 \mathrm{E}+02$ & $4.12 \mathrm{E}+02$ & $2.14 \mathrm{E}+\mathrm{O} 2$ & $2.28 E+02$ \\
\hline $\mathrm{Ni}+3$ & $1.78 E+03$ & $1.24 \mathrm{E}+03$ & $1.65 E+01$ & $1.67 \mathrm{E}+03$ & $1.551 \equiv+02$ & $8.58 E+02$ & $4.20 E+02$ & $4.16 \mathrm{E}+02$ \\
\hline NO2- & $1.60 E+01$ & $7.43 E+00$ & & $5.20 E+01$ & $2.35 E+00$ & $1.55 E+01$ & $7.50 \mathrm{E}+00$ & $1.85 E+01$ \\
\hline No3- & $5.71 E+03$ & $3.02 E+03$ & 2.27E + 01 & $1.62 E+04$ & $7.651 \equiv+02$ & $1.44 \mathrm{E}+02$ & $5.60 E+01$ & $3.41 \mathrm{E}+02$ \\
\hline $\mathrm{OH}-$ & $7.97 E+04$ & $7.18 \mathrm{E}+04$ & $2.06 E+03$ & $3.53 E+04$ & $5.001 \equiv+03$ & $4.78 \mathrm{E}+04$ & $2.29 E+04$ & $2.28 \mathrm{E}+04$ \\
\hline \multicolumn{9}{|l|}{$\mathrm{Pb}+4$} \\
\hline PO4-3 & & & & & & $2.48 \mathrm{E}+\mathrm{O} 2$ & $2.46 \mathrm{E}+\mathrm{O} 2$ & $1.24 E+02$ \\
\hline $\mathrm{Rb}+$ & N/A & N/A & $\mathrm{N} / \mathrm{A}$ & N/A & NIA & $N / A$ & N/A & $\mathrm{N} / \mathrm{A}$ \\
\hline $\mathrm{Re}+7$ & N/A & N/A & N/A & N/A & N/A & $\mathrm{N} / \mathrm{A}$ & N/A & N/A \\
\hline $\mathrm{Rh}+3$ & N/A & N/A & $N / A$ & N/A & N/A & N/A & N/A & $\mathrm{N} / \mathrm{A}$ \\
\hline$R u+3$ & N/A & N/A & N/A & N/A & NIA & N/A & N/A & N/A \\
\hline $\mathrm{Se}+6$ & $\mathrm{~N} / \mathrm{A}$ & N/A & N/A & N/A & N/A & N/A & N/A & N/A \\
\hline \multicolumn{9}{|l|}{$\mathrm{Si}+4$} \\
\hline SO4-2 & $2.77 E+00$ & $2.32 E+00$ & $8.88 \mathrm{E}-02$ & $2.63 E+00$ & 2.3EE-01 & $5.34 \mathrm{E}+00$ & $5.15 E+\infty 0$ & $4.47 \mathrm{E}+00$ \\
\hline $\mathrm{Sr}+2$ & $5.19 E+00$ & $5.99 E+00$ & $1.58 \mathrm{E}+00$ & $5.44 \mathrm{E}+00$ & $3.901 \equiv+\infty 0$ & $2.84 \mathrm{E}+01$ & $2.15 \mathrm{E}-04$ & $1.06 \mathrm{E}-01$ \\
\hline To +6 & N/A & N/A & $N / A$ & N/A & N/A & N/A & $N / A$ & N/A \\
\hline$T h+4$ & N/A & $\mathrm{N} / \mathrm{A}$ & N/A & N/A & NIA & $N / A$ & N/A & N/A \\
\hline $\mathrm{TI}+4$ & N/A & N/A & N/A & N/A & NIA & N/A & N/A & $\mathrm{N} / \mathrm{A}$ \\
\hline TOC & & & & & & & & $2.20 E+00$ \\
\hline $\mathrm{UO} 2+2$ & $1.15 E+04$ & $7.09 E+03$ & $2.69 E+01$ & $1.22 E+04$ & $5.30 \mathrm{OE}+02$ & $1.13 E+04$ & $5.74 \mathrm{E}+03$ & $4.71 \mathrm{E}+03$ \\
\hline$Z n+2$ & N/A & $\mathrm{N} / \mathrm{A}$ & N/A & N/A & $N / A$ & N/A & N/A & $\mathrm{N} / \mathrm{A}$ \\
\hline $\mathrm{ZrO} 2: 2 \mathrm{H} 2 \mathrm{O}$ & & & & & & & & $1.79 E+01$ \\
\hline Total & $1.98 \mathrm{E}+05$ & $1.55 E+05$ & $3.33 E+03$ & $1.58 E+05$ & $1.691 \equiv+04$ & $8.74 E+04$ & $4.21 E+04$ & $4.14 \mathrm{E}+04$ \\
\hline
\end{tabular}


WHC-SD-WM-TI-784

Revision 0

Table A-1. Chemical Inventory Estimates for Single-Shell Tanks

\begin{tabular}{|c|c|c|c|c|c|c|c|c|}
\hline $\begin{array}{c}\text { Insoluble } \\
\text { (kg) }\end{array}$ & $T-104$ & $T-105$ & $T-106$ & $T-107$ & $T-108$ & T-109 & $T-110$ & $T-111$ \\
\hline \multicolumn{9}{|l|}{$\mathrm{Ag}+$} \\
\hline$A l+3$ & $4.98 E+04$ & $6.17 E+01$ & $6.91 E+03$ & $1.80 E+04$ & $2.69 \mathrm{E}+03$ & & & \\
\hline$A s+5$ & N/A & N/A & $N / A$ & N/A & $\mathrm{N} / \mathrm{A}$ & N/A & N/A & N/A \\
\hline$B+3$ & $\mathrm{~N} / \mathrm{A}$ & N/A & N/A & $\mathrm{N} / \mathrm{A}$ & $\mathrm{N} / \mathrm{A}$ & N/A & N/A & N/A \\
\hline $\mathrm{Ba}+2$ & $1.77 \mathrm{E}-01$ & $1.52 \mathrm{E}-01$ & 6.12E-01 & $3.71 \mathrm{E}-01$ & $3.46 \mathrm{E}-01$ & 3.32E-01 & $2.47 \mathrm{E}-04$ & \\
\hline $\mathrm{Be}+2$ & N/A & N/A & $\mathrm{N} / \mathrm{A}$ & N/A & $N / \mu$ & N/A & $N / A$ & N/A \\
\hline $\mathrm{Bi}+3$ & $9.97 E+03$ & $3.22 E+03$ & $1.59 E+02$ & $3.75 E+03$ & $6.00 \mathrm{E}+02$ & $1.14 \mathrm{E}+00$ & $1.49 \mathrm{E}+04$ & $2.10 E+04$ \\
\hline $\mathrm{Ca}+2$ & $1.70 \mathrm{E}+03$ & $6.37 E+02$ & $1.09 E+02$ & $5.90 \mathrm{E}+02$ & $9.82 \mathrm{E}+01$ & $9.71 E+00$ & $3.08 E+03$ & $5.42 \mathrm{E}+03$ \\
\hline Cancrinite & $4.89 E+04$ & $1.96 \mathrm{E}+04$ & $1.73 E+03$ & $1.78 E+04$ & $4.58 \mathrm{E}+03$ & 2.10E + 03 & & \\
\hline $\mathrm{Cd}+2$ & N/A & N/A & N/A & $\mathrm{N} / \mathrm{A}$ & $\mathrm{N} / \mathrm{A}$ & N/A & N/A & $\mathrm{N} / \mathrm{A}$ \\
\hline $\mathrm{Ce}+3$ & $1.15 \mathrm{E}+04$ & & $4.46 \mathrm{E}+01$ & & $1.23 E+01$ & $6.76 E+02$ & & \\
\hline Cl- & $4.54 E+01$ & $1.64 \mathrm{E}+01$ & $1.89 \mathrm{E}+00$ & $1.23 E+01$ & $4.18 \mathrm{E}+00$ & $5.61 E+00$ & $7.05 E+01$ & $8.54 E+01$ \\
\hline $\mathrm{Co}+3$ & N/A & $N / A$ & N/A & $N / A$ & $N / 4$ & N/A & N/A & $\mathrm{N} / \mathrm{A}$ \\
\hline $\cos -2$ & $2.29 E+02$ & & $1.47 \mathrm{E}+01$ & $7.96 \mathrm{E}+01$ & $2.03 E+01$ & $1.91 E+01$ & & $7.32 E+02$ \\
\hline $\mathrm{Cr}+3$ & $1.90 \mathrm{E}+02$ & $4.00 E+01$ & $8.16 E+00$ & $5.06 \mathrm{E}+01$ & $8.29 E+00$ & $4.98 \mathrm{E}-01$ & $1.53 \mathrm{E}+02$ & $2.82 \mathrm{E}+02$ \\
\hline Cst & $6.40 \mathrm{E}-02$ & & $3.62 \mathrm{E}-03$ & & $7.481:-03$ & $3.14 \mathrm{E}-02$ & & \\
\hline $\mathrm{Cu}+2$ & N/A & $N / A$ & $N / A$ & N/A & $N / A$ & N/A & N/A & $\mathrm{N} / \mathrm{A}$ \\
\hline F. & $8.64 E+02$ & $2.37 E+02$ & $1.70 \mathrm{E}+01$ & $2.87 E+02$ & $6.68 \mathrm{E}+01$ & $5.28 \mathrm{E}+01$ & $1.11 E+03$ & $1.18 \mathrm{E}+03$ \\
\hline $\mathrm{Fo}+3$ & $8.82 E+03$ & $3.71 E+03$ & $4.42 \mathrm{E}+02$ & $3.32 \mathrm{E}+03$ & $5.32 \mathrm{E}+02$ & $6.74 \mathrm{E}-01$ & $1.83 \varepsilon+04$ & $3.13 E+04$ \\
\hline $\mathrm{Hg}+2$ & $N / A$ & N/A & N/A & N/A & N/A & N/A & N/A & $N / A$ \\
\hline $\mathrm{K}+$ & & & & & & & $5.47 E+01$ & $1.97 \mathrm{E}+02$ \\
\hline$L a+3$ & & & & & & & $5.78 E+01$ & $2.08 E+02$ \\
\hline $\mathrm{Mg}+2$ & N/A & N/A & N/A & N/A & N/A & $\mathrm{N} / \mathrm{A}$ & N/A & $N / A$ \\
\hline $\mathrm{Mn}+4$ & & & & & & & $2.20 \mathrm{E}+03$ & $7.92 \mathrm{E}+03$ \\
\hline $\mathrm{Mo}+6$ & N/A & N/A & N/A & N/A & $\mathrm{N} / \mathrm{A}$ & N/A & N/A & $N / A$ \\
\hline $\mathrm{Na}+$ & $1.35 E+04$ & $3.03 E+03$ & $4.37 E+02$ & $4.56 \mathrm{E}+03$ & $8.97 E+02$ & $3.99 E+02$ & $7.28 E+04$ & $9.98 \mathrm{E}+03$ \\
\hline $\mathrm{Ni}+3$ & $1.98 E+03$ & $8.48 E+02$ & $1.44 E+02$ & $7.26 E+02$ & $1.18 E+02$ & $3.74 \mathrm{E}+00$ & $4.26 \mathrm{E}+03$ & $7.91 E+03$ \\
\hline No2:- & $1.16 \mathrm{E}+02$ & & $2.04 \mathrm{E}+01$ & $3.11 E+01$ & 1.37E +01 & $2.22 E+01$ & & \\
\hline NO3- & $1.11 E+03$ & & $6.56 \mathrm{E}+01$ & $2.87 \mathrm{E}+02$ & $4.21 E+02$ & $9.51 \mathrm{E}+02$ & & $2.38 \mathrm{E}+03$ \\
\hline $\mathrm{OH}-$ & $4.68 \mathrm{E}+04$ & & $1.29 E+04$ & $1.58 E+04$ & $2.08 \mathrm{E}+03$ & 4.63E-01 & & $2.16 \mathrm{E}+04$ \\
\hline \multicolumn{9}{|l|}{$\mathrm{Pb}+4$} \\
\hline PO4-3 & $1.35 \mathrm{E}+05$ & $2.78 E+04$ & $2.39 E+03$ & $4.81 E+04$ & $7.84 \mathrm{E}+03$ & $3.75 E+02$ & $1.06 E+05$ & $6.65 E+04$ \\
\hline $\mathbf{R b}+$ & N/A & N/A & N/A & N/A & N'A & $\mathrm{N} / \mathrm{A}$ & N/A & N/A \\
\hline $\mathrm{Re}+7$ & N/A & N/A & N/A & N/A & N:A & N/A & N/A & N/A \\
\hline $\mathrm{Rh}+3$ & N/A & $\mathrm{N} / \mathrm{A}$ & N/A & N/A & $N ! A$ & N/A & N/A & $N / A$ \\
\hline $\mathrm{Ru}+3$ & $\mathrm{~N} / \mathrm{A}$ & N/A & $\mathrm{N} / \mathrm{A}$ & $\mathrm{N} / \mathrm{A}$ & N:A & N/A & N/A & $\mathrm{N} / \mathrm{A}$ \\
\hline $5 \theta+6$ & N/A & N/A & N/A & N/A & NIA & N/A & N/A & $\mathrm{N} / \mathrm{A}$ \\
\hline $\mathrm{Si}+4$ & & & & & & $1.12 E+00$ & $4.33 E+03$ & $4.11 E+03$ \\
\hline 504-2 & $5.83 E+01$ & $1.15 E+01$ & $1.55 E+00$ & $1.56 E+01$ & $2.31 E+01$ & $5.21 E+01$ & $3.90 E+01$ & $4.22 E+01$ \\
\hline $\mathrm{Sr}+2$ & $2.00 E-01$ & $2.44 \mathrm{E}-02$ & $7.63 \mathrm{E}+00$ & $1.77 \mathrm{E}-01$ & 1.77 E-O2 & 3.58E-04 & $1.10 \mathrm{E}+03$ & $3.95 E+03$ \\
\hline$T \theta+6$ & $\mathrm{~N} / \mathrm{A}$ & N/A & $N / A$ & N/A & N/A & $N / A$ & N/A & N/A \\
\hline $\mathrm{Th}+4$ & $\mathrm{~N} / \mathrm{A}$ & N/A & N/A & N/A & N/A & N/A & N/A & N/A \\
\hline $\mathrm{TI}+4$ & N/A & N/A & N/A & N/A & $\mathrm{N} / \mathrm{A}$ & $N / A$ & N/A & N/A \\
\hline TOC & & & & & & & $2.84 \mathrm{E}+01$ & $9.86 \mathrm{E}+01$ \\
\hline $402+2$ & $4.60 \mathrm{E}+02$ & $4.83 E+01$ & $1.87 \mathrm{E}+03$ & $5.84 E+01$ & $9.36 \mathrm{E}+00$ & $5.49 \mathrm{E}-02$ & $2.23 E+01$ & $2.35 \mathrm{E}+01$ \\
\hline$Z n+2$ & N/A & $N / A$ & $\mathrm{~N} / \mathrm{A}$ & $N / A$ & N/A & N/A & N/A & $N / A$ \\
\hline ZrO2:2H2O & $1.84 E+04$ & $8.71 \mathrm{E}+02$ & $3.35 E+02$ & $6.45 \mathrm{E}+03$ & $1.03 \mathrm{E}+03$ & & & \\
\hline Total & $3.50 E+05$ & $6.01 E+04$ & $2.76 \mathrm{E}+04$ & $1.20 E+05$ & $2.10 E+04$ & $4.67 E+03$ & $1.68 \mathrm{E}+05$ & $1.85 E+05$ \\
\hline
\end{tabular}


Table A-1. Chemical Inventory Estimates for Single-Shell Tanks

\begin{tabular}{|c|c|c|c|c|c|c|c|c|}
\hline $\begin{array}{c}\text { Insoluble } \\
\text { (kg) }\end{array}$ & $T-112$ & $T-201$ & $T-202$ & $T-203$ & $T-204$ & $T X-101$ & TX-102 & $T X-103$ \\
\hline \multicolumn{9}{|l|}{$\mathrm{Ag}+$} \\
\hline $\mathrm{Al}+3$ & & & & & & $3.68 \mathrm{E}+04$ & $2.05 E+01$ & $2.85 E+01$ \\
\hline$A 8+5$ & N/A & N/A & N/A & N/A & $N / A_{1}$ & N/A & $\mathrm{N} / \mathrm{A}$ & $\mathrm{N} / \mathrm{A}$ \\
\hline$B+3$ & N/A & N/A & N/A & $\mathrm{N} / \mathrm{A}$ & $\mathbf{N} / \boldsymbol{A}_{1}$ & N/A & $\mathrm{N} / \mathrm{A}$ & N/A \\
\hline $\mathrm{Ba}+2$ & $7.68 \mathrm{E}-04$ & & & & & $2.22 \mathrm{E}+00$ & $2.31 E+00$ & $5.75 \mathrm{E}-01$ \\
\hline $\mathrm{B \theta}+2$ & N/A & N/A & N/A & N/A & $\mathbf{N} / \mathbf{A}_{1}$ & N/A & $\mathrm{N} / \mathrm{A}$ & N/A \\
\hline $\mathrm{Bi}+3$ & 3.03E + 03 & $1.05 E+03$ & $7.91 \mathrm{E}+02$ & $1.31 \mathrm{E}+03$ & $1.43 \mathrm{E}+\mathrm{O} 3$ & $9.34 \mathrm{E}-03$ & $1.30 \mathrm{E}-01$ & $1.81 \mathrm{E}-01$ \\
\hline $\mathrm{Ca}+2$ & $8.33 E+02$ & $3.47 E+02$ & $2.61 E+02$ & $4.33 E+02$ & $4.70 \mathrm{E}+02$ & $4.78 E+02$ & $2.32 E+01$ & $3.34 E+01$ \\
\hline Cancrinite & & & & & & $2.14 E+02$ & $2.89 E+03$ & $4.03 E+03$ \\
\hline $\mathrm{Cd}+2$ & N/A & N/A & $N / A$ & N/A & $N / A_{1}$ & N/A & $\mathrm{N} / \mathrm{A}$ & $N / A$ \\
\hline $\mathrm{Ce}+3$ & & & & & & & $4.24 E+00$ & 4.11E+01 \\
\hline $\mathrm{Cl}-$ & $1.11 E+01$ & $5.92 E+00$ & $4.45 \mathrm{E}+00$ & $7.39 \mathrm{E}+\infty 0$ & $8.02 \mathrm{E}+00$ & $2.43 E+01$ & $1.54 E+01$ & $2.15 \mathrm{E}+01$ \\
\hline $\mathrm{Co}+3$ & N/A & N/A & $N / A$ & N/A & $\mathbf{N} / \mathbf{A}_{1}$ & $\mathrm{~N} / \mathrm{A}$ & N/A & $N / A$ \\
\hline $\mathrm{CO}-2$ & $1.12 \mathrm{E}+02$ & $4.68 E+01$ & $3.52 E+01$ & $5.84 E+01$ & $6.35 \mathrm{E}+01$ & $1.04 E+02$ & $4.87 E+01$ & $7.04 \mathrm{E}+01$ \\
\hline $\mathrm{Cr}_{\mathrm{r}+3}$ & 4.55E + 01 & $1.22 \mathrm{E}+01$ & $9.15 \mathrm{E}+00$ & $1.52 \mathrm{E}+01$ & $1.65 \mathrm{E}+01$ & $1.85 E+03$ & $9.88 E+00$ & $1.38 \mathrm{E}+01$ \\
\hline $\mathrm{Cs}_{s+}$ & & & & & & & $1.26 \mathrm{E}-02$ & $1.75 \mathrm{E}-02$ \\
\hline $\mathrm{Cu}+2$ & N/A & $\mathrm{N} / \mathrm{A}$ & N/A & N/A & $\mathbf{N} / \mathbf{A}_{\mathrm{i}}$ & $N / A$ & N/A & N/A \\
\hline F. & $4.60 E+01$ & $4.58 \mathrm{E}+02$ & $3.45 E+02$ & $5.72 E+02$ & $6.22 \mathrm{E}+\mathrm{O} 2$ & $4.34 \mathrm{E}-01$ & $6.02 \mathrm{E}+00$ & $8.39 E+00$ \\
\hline $\mathrm{Fe}+3$ & $4.93 E+03$ & $1.39 \mathrm{E}+03$ & $1.05 E+03$ & $1.74 \mathrm{E}+03$ & $1.89 \mathrm{E}+03$ & $1.95 E+03$ & $3.34 E+01$ & $5.01 E+01$ \\
\hline $\mathrm{Hg}+2$ & N/A & N/A & N/A & $\mathbf{N} / \mathbf{A}$ & $N / A_{1}$ & N/A & $\mathrm{N} / \mathrm{A}$ & $\mathrm{N} / \mathrm{A}$ \\
\hline $\mathrm{K}+$ & & $1.53 \mathrm{E}+02$ & $1.15 E+02$ & $1.91 E+02$ & $2.07 \mathrm{E}+02$ & & & \\
\hline $\mathrm{La}+3$ & & $1.62 \mathrm{E}+02$ & $1.22 \mathrm{E}+\mathrm{O} 2$ & $2.02 \mathrm{E}+02$ & $2.19 \mathrm{E}+\mathrm{O} 2$ & & & \\
\hline $\mathrm{Mg}+2$ & N/A & N/A & N/A & N/A & $N / A_{1}$ & N/A & $\mathrm{N} / \mathrm{A}$ & $\mathrm{N} / \mathrm{A}$ \\
\hline$M n+4$ & & $6.16 \mathrm{E}+03$ & $4.63 E+03$ & $7.69 \mathrm{E}+03$ & $8.35 E+03$ & & & \\
\hline $\mathrm{Mo}+6$ & N/A & N/A & $\mathrm{N} / \mathrm{A}$ & N/A & $\mathrm{N} / \boldsymbol{A}_{1}$ & N/A & $\mathrm{N} / \mathrm{A}$ & N/A \\
\hline $\mathrm{Na}+$ & $8.43 E+02$ & 8.97E + O2 & $6.74 \mathrm{E}+02$ & $1.12 \mathrm{E}+03$ & $1.22 \mathrm{E}+03$ & $1.32 \mathrm{E}+03$ & $1.08 E+03$ & $1.51 E+03$ \\
\hline $\mathrm{Ni}+3$ & $1.24 E+03$ & $5.05 E+02$ & $3.80 \mathrm{E}+\mathrm{O} 2$ & $6.31 \mathrm{E}+0.2$ & $6.85 E+02$ & $6.22 \mathrm{E}+\mathrm{O} 2$ & $1.77 \mathrm{E}+01$ & $2.60 E+01$ \\
\hline NO2- & & & & & & $1.76 \mathrm{E}+01$ & & \\
\hline NO3. & $2.92 E+02$ & $2.14 E+02$ & $1.61 \mathrm{E}+02$ & $2.68 E+02$ & $2.91 \mathrm{E}+02$ & $1.29 \mathrm{E}+03$ & $4.04 E+03$ & $5.73 E+03$ \\
\hline OH. & $5.26 E+03$ & $2.99 \mathrm{E}+03$ & $2.25 E+03$ & $3.73 E+03$ & $4.05 E+03$ & $7.45 \mathrm{E}+04$ & & \\
\hline \multicolumn{9}{|l|}{$\mathrm{Pb}+4$} \\
\hline PO4-3 & $4.99 E+03$ & $1.16 \mathrm{E}+03$ & $8.70 E+02$ & $1.45 E+03$ & $1.57 \varepsilon+03$ & $3.72 \mathrm{E}+02$ & $2.64 E+02$ & $3.96 E+02$ \\
\hline $\mathrm{Rb}+$ & N/A & N/A & $\mathrm{N} / \mathrm{A}$ & $\mathrm{N} / \mathrm{A}$ & $N / A_{1}$ & N/A & N/A & N/A \\
\hline $\mathrm{Re}+7$ & N/A & N/A & N/A & $\mathrm{N} / \mathrm{A}$ & $N / A_{i}$ & N/A & N/A & N/A \\
\hline$R h+3$ & N/A & N/A & N/A & N/A & $\mathrm{N} / \mathrm{f}_{1}$ & N/A & N/A & N/A \\
\hline $\mathrm{Ru}+3$ & N/A & $N / A$ & $N / A$ & $N / A$ & $N / A$ & N/A & N/A & N/A \\
\hline $\mathrm{Se}+6$ & N/A & $N / A$ & N/A & N/A & $N / A$ & $\mathrm{~N} / \mathrm{A}$ & N/A & N/A \\
\hline $\mathrm{Si}+4$ & $5.22 \mathrm{E}+02$ & & & & & & & \\
\hline SO4-2 & $5.76 \mathrm{E}+00$ & 2.63E-01 & $1.98 \mathrm{E}-01$ & $3.28 \mathrm{E}-01$ & 3.57E-01 & $1.36 E+01$ & $4.51 E+01$ & $6.34 E+01$ \\
\hline $\mathrm{Sr}+2$ & $2.29 \mathrm{E}-02$ & $3.08 \mathrm{E}+03$ & $2.31 E+03$ & $3.84 \mathrm{E}+03$ & 4.17E+O3 & $4.29 \mathrm{E}-\mathrm{O} 2$ & $1.64 \mathrm{E}-03$ & $3.65 \mathrm{E}-03$ \\
\hline $\mathrm{Te}+6$ & N/A & $N / A$ & N/A & N/A & $\mathrm{N} / \mathrm{f}$ & $N / A$ & N/A & N/A \\
\hline$T h+4$ & N/A & N/A & N/A & N/A & $N / A$ & $\mathrm{~N} / \mathrm{A}$ & $N / A$ & N/A \\
\hline$T I+4$ & N/A & N/A & N/A & N/A & $\mathrm{N} / \mathrm{A}$ & N/A & $\mathrm{N} / \mathrm{A}$ & $N / A$ \\
\hline Toc & & $8.49 \mathrm{E}+01$ & $6.39 \mathrm{E}+01$ & $1.06 \mathrm{E}+02$ & $1.15 E+02$ & $3.24 \mathrm{E}-01$ & $5.34 E+00$ & $7.44 E+00$ \\
\hline $402+2$ & $3.20 E+00$ & & & & & $4.11 \mathrm{E}+03$ & $2.12 \mathrm{E}+03$ & $3.19 E+03$ \\
\hline$Z n+2$ & N/A & N/A & N/A & $\mathrm{N} / \mathrm{A}$ & $N / 4$ & $N / A$ & N/A & N/A \\
\hline \multicolumn{9}{|l|}{$\mathrm{ZrO} 2: 2 \mathrm{H} 2 \mathrm{O}$} \\
\hline Total & $2.22 E+04$ & $1.87 E+04$ & $1.41 E+04$ & $2.34 E+04$ & $2.54 \mathrm{E}+04$ & $1.24 E+05$ & $1.06 \mathrm{E}+04$ & $1.52 \mathrm{E}+04$ \\
\hline
\end{tabular}


WHC-SD-WM-TI-784

Revision 0

Table A-1. Chemical Inventory Estimates for Single-Shell Tanks

\begin{tabular}{|c|c|c|c|c|c|c|c|c|}
\hline $\begin{array}{c}\text { Insoluble } \\
\text { (kg) }\end{array}$ & $T X-104$ & TX-105 & TX-106 & $T \times-107$ & $T X-11) 8$ & $T X-109$ & $\mathrm{TX}-110$ & TX-111 \\
\hline \multicolumn{9}{|l|}{$A_{Q}+$} \\
\hline $\mathrm{Al}+3$ & $8.48 E+00$ & t.11E+O2 & $1.58 E+03$ & $4.98 E+00$ & $2.36 E-01$ & $5.30 E+04$ & $4.01 E+03$ & $4.02 E+03$ \\
\hline$A 8+5$ & N/A & N/A & N/A & N/A & $N / A$ & N/A & N/A & N/A \\
\hline$B+3$ & N/A & $N / A$ & $\mathrm{~N} / \mathrm{A}$ & $\mathrm{N} / \mathrm{A}$ & $N / A$ & N/A & N/A & $N / A$ \\
\hline $\mathrm{Ba}+2$ & $1.07 E+00$ & $3.83 E+00$ & $1.33 \mathrm{E}-01$ & & $1.22 \mathrm{E}+00$ & $2.62 \mathrm{E}+00$ & $1.29 \mathrm{E}+00$ & $6.95 \mathrm{E}-01$ \\
\hline $\mathrm{Be}+2$ & N/A & N/A & N/A & N/A & $N / A$ & N/A & N/A & $N / A$ \\
\hline $\mathrm{Bi}+3$ & $5.37 \mathrm{E}-02$ & 7.03E-01 & $5.23 E-01$ & $3.15 \mathrm{E}-\mathrm{O} 2$ & $1.50 \mathrm{E} 01$ & $1.10 E+O 4$ & $7.05 E+02$ & $6.85 E+02$ \\
\hline $\mathrm{Ca}+2$ & $9.33 E+01$ & $1.12 E+02$ & $7.68 E+01$ & $4.23 E+01$ & $3.24 E+02$ & $1.73 E+03$ & $1.80 E+02$ & $1.74 \mathrm{E}+\mathrm{O} 2$ \\
\hline Cancrinite & $1.23 E+03$ & $1.57 E+04$ & $1.16 E+04$ & $7.13 E+02$ & $3.34 E+03$ & $5.23 E+04$ & $1.46 \mathrm{E}+04$ & $1.21 E+04$ \\
\hline $\mathrm{Cd}+2$ & $N / A$ & N/A & $\mathrm{N} / \mathrm{A}$ & N/A & N/A & N/A & N/A & N/A \\
\hline $\mathrm{Ce}+3$ & & $4.98 E+01$ & & & $2.53 E+04$ & $2.17 \mathrm{E}+04$ & $1.69 E+04$ & $4.41 E+03$ \\
\hline $\mathrm{Cl}-$ & $6.74 E+00$ & $8.34 E+01$ & $6.29 E+01$ & $3.85 E+00$ & $2.63 E+01$ & $3.61 \mathrm{E}+01$ & $6.29 \mathrm{E}+01$ & $5.01 E+01$ \\
\hline $\mathrm{Co}+3$ & $N / A$ & $\mathrm{~N} / \mathrm{A}$ & $N / A$ & N/A & $N / A$ & N/A & N/A & $\mathrm{N} / \mathrm{A}$ \\
\hline $\mathrm{CO} 3-2$ & $2.07 E+02$ & $2.62 E+02$ & $1.20 E+02$ & $1.22 E+02$ & $1.49 \mathrm{E}+02$ & $2.34 \mathrm{E}+02$ & $1.21 E+02$ & $9.77 E+01$ \\
\hline $\mathrm{Cr}+3$ & $8.21 E+00$ & $5.23 E+01$ & $1.18 \mathrm{E}+02$ & $3.63 E+00$ & $1.90 E+01$ & $1.49 \mathrm{E}+02$ & $5.29 \mathrm{E}+01$ & $4.81 E+01$ \\
\hline Cs + & & $1.22 \mathrm{E}-01$ & $1.97 \mathrm{E}-01$ & & 7.31E 01 & $5.25 \mathrm{E}+00$ & $2.20 E+00$ & $4.99 \mathrm{E}-01$ \\
\hline $\mathrm{Cu}+2$ & N/A & N/A & $\mathrm{N} / \mathrm{A}$ & $N / A$ & $N / A$ & N/A & $\mathrm{N} / \mathrm{A}$ & N/A \\
\hline F- & $2.50 E+00$ & $3.26 \varepsilon+01$ & $2.43 E+01$ & $1.46 \mathrm{E}+00$ & $6.95 \mathrm{E}+00$ & $8.44 E+02$ & $9.03 E+01$ & $9.05 E+01$ \\
\hline $\mathrm{Fe}+3$ & $2.92 \mathrm{E}+02$ & $1.35 E+02$ & $9.98 E+01$ & $1.30 E+02$ & $6.07 \mathrm{E}+\mathrm{O} 2$ & $9.76 \mathrm{E}+03$ & $6.24 \mathrm{E}+02$ & $6.04 E+02$ \\
\hline $\mathrm{Hg}+2$ & N/A & $\mathrm{N} / \mathrm{A}$ & N/A & $\mathrm{N} / \mathrm{A}$ & N/A & N/A & N/A & N/A \\
\hline \multicolumn{9}{|l|}{$\mathrm{K}+$} \\
\hline \multicolumn{9}{|l|}{$\mathrm{La}+3$} \\
\hline $\mathrm{Mg}+2$ & N/A & N/A & $N / A$ & N/A & N/A & N/A & $\mathrm{N} / \mathrm{A}$ & N/A \\
\hline \multicolumn{9}{|l|}{$M n+4$} \\
\hline Mo+6 & N/A & N/A & N/A & N/A & $\mathrm{N} / \mathrm{A}$ & N/A & $\mathrm{N} / \mathrm{A}$ & $\mathrm{N} / \mathrm{A}$ \\
\hline $\mathrm{Na}+$ & $1.01 E+03$ & $5.78 \mathrm{E}+03$ & $4.16 E+03$ & $5.34 \mathrm{E}+02$ & $1.93 E+03$ & $1.34 E+04$ & $4.94 E+03$ & $4.11 E+03$ \\
\hline $\mathrm{Ni}+3$ & $1.14 E+02$ & $7.81 \mathrm{E}+01$ & $5.02 \mathrm{E}+01$ & $5.12 \mathrm{E}+01$ & $3.29 \mathrm{E}+\mathrm{O} 2$ & $2.13 E+03$ & $1.62 E+02$ & $1.57 E+02$ \\
\hline NO2- & $1.01 \mathrm{E}+02$ & & & $5.92 \mathrm{E}+01$ & $2.80 \mathrm{E}+02$ & $9.13 E+01$ & $9.42 E+02$ & $7.28 \mathrm{E}+02$ \\
\hline No3. & $2.63 E+03$ & $2.30 E+04$ & $2.53 E+04$ & $1.54 E+03$ & $7.34 \mathrm{E}+03$ & $8.44 E+02$ & $2.43 E+04$ & $1.87 E+04$ \\
\hline $\mathrm{OH}-$ & $1.41 E+03$ & & & $1.31 E+03$ & $9.43 \mathrm{E}+03$ & $5.44 \mathrm{E}+04$ & $5.7 O E+O 3$ & $2.02 E+03$ \\
\hline \multicolumn{9}{|l|}{$\mathrm{Pb}+4$} \\
\hline PO4-3 & $2.23 E+03$ & $1.08 \mathrm{E}+03$ & $1.62 \mathrm{E}+02$ & $9.92 E+02$ & $8.82 \mathrm{E}+02$ & $1.41 \mathrm{E}+05$ & $1.01 E+04$ & $1.03 E+04$ \\
\hline $\mathrm{Rb}+$ & N/A & N/A & N/A & N/A & $N / A_{1}$ & N/A & $\mathrm{N} / \mathrm{A}$ & N/A \\
\hline$R e+7$ & N/A & $\mathrm{N} / \mathrm{A}$ & N/A & N/A & $N / A$ & N/A & $\mathrm{N} / \mathrm{A}$ & N/A \\
\hline $\mathrm{Rh}+3$ & N/A & $\mathrm{N} / \mathrm{A}$ & $\mathrm{N} / \mathrm{A}$ & $N / A$ & $N / A$ & N/A & N/A & $N / A$ \\
\hline $\mathrm{Ru}+3$ & $\mathrm{~N} / \mathrm{A}$ & N/A & N/A & N/A & $\mathrm{N} / \mathrm{A}_{\mathrm{A}}$ & N/A & $N / A$ & N/A \\
\hline $\mathrm{Se}+6$ & $\mathrm{~N} / \mathrm{A}$ & $\mathrm{N} / \mathrm{A}$ & N/A & N/A & $N / A_{1}$ & N/A & N/A & N/A \\
\hline \multicolumn{9}{|l|}{$\mathrm{Si}+4$} \\
\hline SO4-2 & $6.19 \mathrm{E}+01$ & $2.38 E+02$ & $1.64 \mathrm{E}+02$ & $2.99 E+01$ & $7.81 \mathrm{E}+01$ & $4.57 E+01$ & $1.59 E+02$ & $1.24 \mathrm{E}+02$ \\
\hline $\mathrm{Sr}+2$ & & $1.61 \mathrm{E}-02$ & & & $2.58 \mathrm{E} \cdot 02$ & $7.40 E-01$ & $5.19 \mathrm{E}-01$ & $2.45 \mathrm{E}-01$ \\
\hline$T \theta+6$ & $\mathrm{~N} / \mathrm{A}$ & N/A & N/A & N/A & $N / A_{1}$ & $\mathrm{~N} / \mathrm{A}$ & $\mathrm{N} / \mathrm{A}$ & $N / A$ \\
\hline$T h+4$ & N/A & N/A & N/A & N/A & $\mathrm{N} / \mathrm{A}_{\mathrm{i}}$ & N/A & $\mathrm{N} / \mathrm{A}$ & N/A \\
\hline $\mathrm{TI}+4$ & $\mathrm{~N} / \mathrm{A}$ & N/A & N/A & N/A & $\mathrm{N} / A_{i}$ & N/A & N/A & N/A \\
\hline TOC & $2.15 E+00$ & $2.90 \mathrm{E}+01$ & $2.15 E+01$ & $1.27 E+00$ & $6.13 E+00$ & & $2.01 E+01$ & $1.53 E+01$ \\
\hline $\mathrm{uO} 2+2$ & $1.91 E+04$ & $1.44 \mathrm{E}+04$ & $8.49 E+02$ & $1.44 E+04$ & $5.13 E+03$ & 1.71E + 02 & $5.69 \mathrm{E}+01$ & $7.68 \mathrm{E}+01$ \\
\hline $2 n+2$ & N/A & $\mathrm{N} / \mathrm{A}$ & $N / A$ & $N / A$ & $\mathrm{~N} / \mathrm{A}_{1}$ & N/A & $N / A$ & $N / A$ \\
\hline $\mathrm{ZrO} 2: 2 \mathrm{H} 2 \mathrm{O}$ & & & & & & $1.89 E+04$ & $1.38 \mathrm{E}+03$ & $1.44 \mathrm{E}+03$ \\
\hline Total & $2.85 \varepsilon+04$ & $6.12 E+04$ & $4.44 E+0.4$ & $1.99 E+04$ & $5.52 \mathrm{E}+04$ & $3.82 E+05$ & $8.52 E+04$ & $6.00 E+04$ \\
\hline
\end{tabular}


Table A-1. Chemical Inventory Estimates for Single-Shell Tanks

\begin{tabular}{|c|c|c|c|c|c|c|c|c|}
\hline $\begin{array}{c}\text { Insoluble } \\
(\mathrm{kg})\end{array}$ & $T X-112$ & $T X-113$ & $T X-114$ & $T X-115$ & $\mathrm{Tx}-116$ & $T X-117$ & $T X-118$ & TY-101 \\
\hline $\mathrm{Ag}+$ & & & & & & & $1.56 \mathrm{E}-05$ & \\
\hline$A l+3$ & $1.15 E+02$ & $2.67 E+04$ & $5.66 \mathrm{E}+02$ & $1.17 E+02$ & $5.07 E+01$ & $7,64 \mathrm{E}+01$ & $1.21 E+04$ & \\
\hline$A s+5$ & $\mathrm{~N} / \mathrm{A}$ & N/A & N/A & N/A & $N / A$ & $N / A$ & $N / A$ & N/A \\
\hline$B+3$ & N/A & N/A & N/A & N/A & N/A & $N / A$ & N/A & $\mathrm{N} / \mathrm{A}$ \\
\hline $\mathrm{Ba}+2$ & $8.09 E-01$ & 3.63E-01 & 9.81E-01 & $1.80 \mathrm{E}-01$ & $4.71 \mathrm{E} 01$ & $4.64 E-01$ & $8.94 E+00$ & $3.78 \mathrm{E}-01$ \\
\hline $\mathrm{Be}+2$ & N/A & N/A & N/A & N/A & $\mathrm{N} / \mathrm{A}$ & N/A & N/A & N/A \\
\hline $\mathrm{Bi}+3$ & $1.20 E+00$ & $5.23 E+03$ & $1.16 \mathrm{E}+02$ & $7.38 \mathrm{E}-01$ & $6.66 \mathrm{E}+00$ & $4.35 E+00$ & $4.78 \varepsilon-01$ & $4.30 E+O 3$ \\
\hline $\mathrm{Ca}+2$ & $7.99 E+01$ & $8.74 E+02$ & $7.34 E+01$ & $2.17 E+02$ & $8.89 E+01$ & $8.41 E+01$ & $2.74 \mathrm{E}+02$ & $7.94 E+02$ \\
\hline Cancrinite & $1.81 E+04$ & $3.59 E+04$ & $1.72 E+04$ & $1.64 E+04$ & $3.18 \mathrm{E}+04$ & $2.58 \mathrm{E}+04$ & $8.48 \mathrm{E}+03$ & $1.67 E+03$ \\
\hline $\mathrm{Cd}+2$ & $N / A$ & N/A & N/A & N/A & $N / A$ & $N / A$ & N/A & N/A \\
\hline $\mathrm{Co}+3$ & $2.23 E+04$ & $1.44 E+04$ & $4.00 E+03$ & $6.19 E+03$ & $3.34 \mathrm{E}+03$ & $1.35 E+03$ & $1.39 E+04$ & $5.61 E+02$ \\
\hline $\mathrm{Cl}-$ & $8.89 E+01$ & $7.58 E+01$ & $7.09 E+01$ & $9.22 E+01$ & $7.10 \mathrm{E}+01$ & $7.74 \mathrm{E}+01$ & $6.99 \mathrm{E}+01$ & $7.54 \mathrm{E}+00$ \\
\hline $\mathrm{Co}+3$ & N/A & $N / A$ & $N / A$ & N/A & $\mathbf{N} / \mathbf{A}$ & $N / A$ & N/A & N/A \\
\hline $\mathrm{CO} 3-2$ & $1.60 E+02$ & $2.14 E+02$ & $1.35 \mathrm{E}+\mathrm{O} 2$ & $1.87 E+02$ & $1.76 E+02$ & $1.68 \mathrm{E}+02$ & $1.82 \mathrm{E}+02$ & \\
\hline $\mathrm{Cr}+3$ & $5.32 \mathrm{E}+01$ & $1.06 E+02$ & $3.53 E+01$ & $5.62 E+01$ & $2.72 \mathrm{E}+01$ & $3.74 \mathrm{E}+01$ & $4.21 \mathrm{E}+01$ & $2.72 \mathrm{E}+00$ \\
\hline $\mathrm{Cs}+$ & $3.87 \mathrm{E}-01$ & $1.33 \mathrm{E}-01$ & $3.86 \mathrm{E}-01$ & $2.16 \mathrm{E}-01$ & $9.18 \mathrm{E} 02$ & $3.72 \mathrm{E}-02$ & $6.12 \mathrm{E}+00$ & $3.57 \mathrm{E}-02$ \\
\hline $\mathrm{Cu}+2$ & $\mathrm{~N} / \mathrm{A}$ & $\mathrm{N} / \mathrm{A}$ & N/A & N/A & $N / A$ & N/A & N/A & N/A \\
\hline F- & $5.58 E+01$ & $4.24 E+02$ & $8.70 E+01$ & $3.43 E+01$ & $3.09 E+02$ & $2.02 \mathrm{E}+02$ & $2.22 \mathrm{E}+01$ & $2.88 \mathrm{E}+02$ \\
\hline $\mathrm{Fe}+3$ & $5.55 E+00$ & $4.64 E+03$ & $1.05 E+02$ & $9.94 E+02$ & $6.17 \mathrm{E}+00$ & $5.84 \mathrm{E}+00$ & $8.19 E+02$ & $1.87 E+03$ \\
\hline $\mathrm{Hg}+2$ & $N / A$ & $N / A$ & N/A & N/A & $N / A$ & $\mathrm{~N} / \mathrm{A}$ & N/A & N/A \\
\hline \multicolumn{9}{|l|}{$K+$} \\
\hline \multicolumn{9}{|l|}{$L a+3$} \\
\hline $\mathrm{Mg}+2$ & N/A & N/A & $\mathrm{N} / \mathrm{A}$ & N/A & $\mathbf{N} / \mathbf{A}$ & $N / A$ & $\mathrm{~N} / \mathrm{A}$ & $\mathrm{N} / \mathrm{A}$ \\
\hline \multicolumn{9}{|l|}{$M n+4$} \\
\hline $\mathrm{Mo}+6$ & N/A & N/A & $\mathrm{N} / \mathrm{A}$ & N/A & $N / A$ & N/A & N/A & N/A \\
\hline $\mathrm{Na}+$ & $5 . \mathbf{8 8 E}+03$ & $1.04 E+04$ & 4.77E+03 & $6.02 E+03$ & $4.83 \mathrm{E}+03$ & $5.20 \mathrm{E}+03$ & $3.62 \mathrm{E}+\mathrm{O3}$ & $5.36 E+03$ \\
\hline $\mathrm{Ni}+3$ & $3.07 E+01$ & $1.03 E+03$ & $4.34 E+01$ & $2.29 E+02$ & $3.42 \mathrm{E}+01$ & $3.24 E+01$ & $3.15 E+02$ & $2.86 E+03$ \\
\hline NO2- & $1.38 \mathrm{E}+03$ & $9.71 E+02$ & & & & & $6.78 \mathrm{E}+02$ & \\
\hline NO3. & $3.60 \mathrm{E}+04$ & $2.45 E+04$ & $2.15 E+04$ & $3.31 E+04$ & $1.52 \mathrm{E}+04$ & $1.60 \mathrm{E}+04$ & $1.81 E+04$ & \\
\hline $\mathrm{OH}$ - & $1.97 \mathrm{E}+03$ & $2.62 E+04$ & & & & & $2.61 E+04$ & \\
\hline \multicolumn{9}{|l|}{$\mathrm{Pb}+4$} \\
\hline PO4-3 & $2.51 E+02$ & $6.71 E+04$ & $1.91 \mathrm{E}+03$ & $2.92 \mathrm{E}+02$ & $2.13 E+03$ & $1.34 \mathrm{E}+03$ & $8.71 E+01$ & $5.87 E+04$ \\
\hline $\mathrm{Rb}+$ & N/A & N/A & N/A & N/A & $\mathbf{N} / \mathbf{A}$ & N/A & $N / A$ & $N / A$ \\
\hline$R e+7$ & N/A & N/A & N/A & N/A & N/A & N/A & N/A & N/A \\
\hline$R h+3$ & N/A & $N / A$ & N/A & N/A & $N / A$ & $\mathrm{~N} / \mathrm{A}$ & N/A & N/A \\
\hline$R u+3$ & N/A & N/A & $N / A$ & N/A & $N / A$ & N/A & N/A & $\mathrm{N} / \mathrm{A}$ \\
\hline $\mathrm{Se}+6$ & N/A & N/A & $N / A$ & N/A & $N / A$ & N/A & N/A & $\mathrm{N} / \mathrm{A}$ \\
\hline$S i+4$ & & & & & & & & $8.81 E+02$ \\
\hline so4-2 & $2.48 \mathrm{E}+02$ & $1.75 \mathrm{E}+\mathrm{O} 2$ & $2.23 E+02$ & $2.50 \mathrm{E}+\mathrm{O} 2$ & $3.94 \mathrm{E}+02$ & $3.29 \mathrm{E}+0.2$ & $1.17 \mathrm{E}+02$ & $4.55 E+01$ \\
\hline $\mathrm{Sr}+2$ & 3.88E-02 & 4.16E-O2 & 3.89E-03 & $1.52 \mathrm{E}-01$ & $1.97 \mathrm{E} 01$ & $3.75 \mathrm{E}-01$ & $3.86 E+00$ & $8.61 \mathrm{E}-02$ \\
\hline $\mathrm{Te}+6$ & $N / A$ & N/A & N/A & N/A & $N / A$ & N/A & N/A & $\mathrm{N} / \mathrm{A}$ \\
\hline$T h+4$ & $\mathrm{~N} / \mathrm{A}$ & N/A & N/A & N/A & $N / A_{1}$ & N/A & $N / A$ & $N / A$ \\
\hline $\mathrm{TI}+4$ & N/A & N/A & $\mathrm{N} / \mathrm{A}$ & N/A & $N / A_{1}$ & $\mathrm{~N} / \mathrm{A}$ & N/A & $\mathrm{N} / \mathrm{A}$ \\
\hline TOC & $3.01 E+01$ & $1.92 \mathrm{E}+01$ & $2.21 E+01$ & $3.04 E+01$ & $1.33 \mathrm{E}+01$ & $1.98 \mathrm{E}+01$ & $1.45 E+01$ & $2.37 E+02$ \\
\hline $\mathrm{UO} 2+2$ & $8.12 E+00$ & $8.69 E+01$ & $6.18 E+00$ & $1.17 E+03$ & $4.03 E+00$ & $5.65 E+00$ & $4.00 E+00$ & $4.14 \mathrm{E}+01$ \\
\hline $2 n+2$ & N/A & N/A & N/A & N/A & $N / A_{1}$ & N/A & N/A & N/A \\
\hline $\mathrm{ZrO} 2: 2 \mathrm{H} 2 \mathrm{O}$ & & $8.99 E+03$ & $1.93 E+02$ & & & & & $6.32 \mathrm{E}+03$ \\
\hline Total & $8.68 E+04$ & $2.28 \mathrm{E}+05$ & $5.11 E+04$ & $6.54 \mathrm{E}+04$ & $5.85 \mathrm{E}+04$ & $5.08 E+04$ & $8.50 \varepsilon+04$ & $8.39 E+04$ \\
\hline
\end{tabular}


WHC-SD-WM-TI-784

Revision 0

Table A-1. Chemical Inventory Estimates for Single-Shell Tanks

\begin{tabular}{|c|c|c|c|c|c|c|c|c|}
\hline $\begin{array}{c}\text { Insoluble } \\
(\mathrm{kg})\end{array}$ & TY-102 & TY-103 & TY-104 & TY-105 & TY-1016 & U-101 & U-102 & $\mathrm{U}-103$ \\
\hline \multicolumn{9}{|l|}{$\mathrm{Ag}+$} \\
\hline$A \mathrm{I}+3$ & $6.25 E+00$ & $7.54 E+00$ & & & & & $7.11 E+01$ & $1.45 \mathrm{E}+02$ \\
\hline As +5 & N/A & $N / A$ & N/A & N/A & N/A & N/A & N/A & $N / A$ \\
\hline $\mathrm{B}+3$ & N/A & N/A & N/A & N/A & N/A & $\mathrm{N} / \mathrm{A}$ & N/A & $N / A$ \\
\hline $\mathrm{Ba}+2$ & $9.94 \mathrm{E}-01$ & $1.65 E+\infty 0$ & 2.46E-01 & $1.55 E+O 0$ & 3.17E-01 & $7.90 \mathrm{E}-01$ & $1.39 E+00$ & $2.39 E+00$ \\
\hline $\mathrm{Be}+2$ & N/A & N/A & N/A & N/A & $N / A$ & N/A & N/A & N/A \\
\hline $\mathrm{Bi}+3$ & 6.10E-01 & $2.81 E+03$ & & & & & 4.97E-01 & $7.71 E-01$ \\
\hline $\mathrm{Ca}+2$ & $9.11 E+00$ & $1.59 E+03$ & $7.57 \mathrm{E}+02$ & $2.78 \mathrm{E}+03$ & $2.82 E+.02$ & $1.07 E+02$ & $1.89 E+02$ & $1.86 \mathrm{E}+02$ \\
\hline Cancrinite & $3.10 E+03$ & $1.39 E+04$ & & & & & $2.28 E+04$ & $1.23 E+04$ \\
\hline $\mathrm{Cd}+2$ & N/A & N/A & N/A & $N / A$ & N/A & $\mathrm{N} / \mathrm{A}$ & N/A & N/A \\
\hline $\mathrm{Ce}+3$ & $4.38 E+02$ & $2.76 E+O 2$ & $2.27 E+02$ & $2.79 E+O O$ & & & $4.93 E+03$ & $1.40 \mathrm{E}-02$ \\
\hline $\mathrm{Cl}-$ & $7.66 \mathrm{E}+00$ & $4.58 E+01$ & $2.56 \mathrm{E}+01$ & $9.39 E+01$ & $9.52 E+00$ & 4.53E-01 & $1.85 E+02$ & $2.7 \mathrm{OE}+02$ \\
\hline $\mathrm{Co}+3$ & N/A & N/A & $N / A$ & N/A & $\mathrm{N} / \mathrm{A}$ & N/A & N/A & N/A \\
\hline $\mathrm{CO}-2$ & $1.81 E+01$ & & $1.80 E+02$ & $6.60 \mathrm{E}+02$ & $6.69 E+.01$ & $2.38 \mathrm{E}+\mathrm{O} 2$ & $5.26 E+02$ & $7.39 \mathrm{E}+02$ \\
\hline $\mathrm{Cr}+3$ & $3.22 \mathrm{E}+00$ & $2.64 \mathrm{E}+01$ & $1.43 E+01$ & $5.25 \mathrm{E}+01$ & $5.32 E+.00$ & $5.26 \mathrm{E}+00$ & $6.54 E+01$ & $2.41 E+01$ \\
\hline Cs + & 4.11E-01 & $8.22 \mathrm{E}-01$ & $9.35 \mathrm{E}-03$ & $1.63 \mathrm{E}-01$ & & $3.54 \mathrm{E}-01$ & 8.90E-01 & $1.46 \mathrm{E}-04$ \\
\hline $\mathrm{Cu}+2$ & N/A & N/A & N/A & N/A & N/A & N/A & N/A & N/A \\
\hline F. & $2.83 E+01$ & $1.64 \mathrm{E}+02$ & & & & & $2.31 \mathrm{E}+01$ & $3.58 E+01$ \\
\hline $\mathrm{Fe}+3$ & $6.32 \mathrm{E}-01$ & $8.77 E+03$ & $5.32 \mathrm{E}+03$ & $1.95 E+04$ & $1.98 E+03$ & $3.55 \mathrm{E}+02$ & $6.50 E+02$ & $5.20 E+02$ \\
\hline $\mathrm{Hg}+2$ & N/A & N/A & N/A & $N / A$ & N/A & N/A & N/A & N/A \\
\hline $\mathrm{K}+$ & & & & & & & $4.12 \mathrm{E}+00$ & $1.55 E+01$ \\
\hline \multicolumn{9}{|l|}{$\mathrm{La}+3$} \\
\hline $\mathrm{Mg}+2$ & N/A & N/A & N/A & N/A & $\mathrm{N} / \mathrm{A}$ & N/A & N/A & N/A \\
\hline $\mathrm{Mn}+4$ & & & & & & & $5.34 \mathrm{E}+02$ & $2.01 E+03$ \\
\hline $\mathrm{Mo}+6$ & $\mathrm{~N} / \mathrm{A}$ & N/A & $N / A$ & N/A & N/A & N/A & N/A & $N / A$ \\
\hline $\mathrm{Na}+$ & $5.18 E+02$ & $5.63 E+03$ & $1.35 E+03$ & $4.96 \mathrm{E}+03$ & $5.02 E+02$ & $7.19 E+02$ & $4.31 E+03$ & $4.34 E+03$ \\
\hline $\mathrm{Ni}+3$ & $3.50 E+00$ & $3.39 E+03$ & $1.07 E+03$ & $3.94 E+03$ & $3.99 \mathrm{E}+02$ & $1.37 E+02$ & $2.22 \mathrm{E}+02$ & $2.11 \mathrm{E}+\mathrm{O} 2$ \\
\hline $\mathrm{NO} 2-$ & & & & & & & $9.24 \mathrm{E}+02$ & $1.48 \mathrm{E}+03$ \\
\hline NO3- & $1.84 \mathrm{E}+03$ & & $4.89 \mathrm{E}+02$ & $1.80 E+03$ & $1.82 \mathrm{E}+02$ & $1.60 E+01$ & $1.42 E+04$ & $1.28 E+04$ \\
\hline $\mathrm{OH}-$ & & & $6.67 \mathrm{E}+03$ & $2.42 \mathrm{E}+04$ & $2.45 E+03$ & $2.26 \mathrm{E}+03$ & $7.36 E+03$ & $1.55 E+03$ \\
\hline $\mathrm{Pb}+4$ & & & & & & & $6.19 E-04$ & $2.33 \mathrm{E}-03$ \\
\hline PO4-3 & $1.93 E+02$ & $3.96 \mathrm{E}+04$ & $1.05 E+03$ & $3.85 \mathrm{E}+03$ & $3.90 \mathrm{E}+02$ & $2.71 E+03$ & $7.30 E+02$ & $4.09 E+03$ \\
\hline $\mathrm{Rb}+$ & N/A & N/A & N/A & N/A & N/A & N/A & N/A & N/A \\
\hline $\operatorname{Re}+7$ & N/A & N/A & N/A & N/A & N/A & N/A & N/A & N/A \\
\hline$R h+3$ & $\mathrm{~N} / \mathrm{A}$ & N/A & N/A & N/A & $\mathrm{N} / \mathrm{A}$ & N/A & N/A & $N / A$ \\
\hline$R u+3$ & N/A & N/A & N/A & N/A & $\mathrm{N} / \mathrm{A}$ & $N / A$ & N/A & N/A \\
\hline $\mathrm{Se}+6$ & N/A & $N / A$ & N/A & N/A & $\mathrm{N} / \mathrm{A}$ & N/A & $N / A$ & N/A \\
\hline $5 i+4$ & & & & & & $7.43 \mathrm{E}-01$ & & \\
\hline so4-2 & $3.87 E+01$ & $1.88 \mathrm{E}+02$ & $1.17 E+02$ & $4.30 E+02$ & $4.35 E+01$ & $5.51 E+01$ & $1.92 \mathrm{E}+02$ & $2.18 E+02$ \\
\hline $\mathrm{Sr}+2$ & $6.09 \mathrm{E}-02$ & $5.49 E-01$ & $3.11 \mathrm{E}-02$ & $1.29 \varepsilon+00$ & $4.73 \mathrm{E}-\mathrm{O} 2$ & 6.75E-02 & 2.58E-01 & $1.06 \mathrm{E}-05$ \\
\hline $\mathrm{Te}+6$ & N/A & N/A & N/A & N/A & $\mathrm{N} / \mathrm{A}$ & N/A & N/A & $N / A$ \\
\hline$T h+4$ & N/A & N/A & $N / A$ & N/A & $\mathrm{N} / \mathrm{A}$ & N/A & N/A & N/A \\
\hline$T I+4$ & N/A & $N / A$ & $N / A$ & N/A & $N / A$ & N/A & N/A & N/A \\
\hline TOC & $1.63 E+00$ & $1.55 E+02$ & & & & & $4.16 E+01$ & $9.09 E+01$ \\
\hline$\cup 02+2$ & $4.81 \mathrm{E}-01$ & $8.86 \mathrm{E}+03$ & $6.23 \mathrm{E}+03$ & $2.29 \mathrm{E}+\mathrm{O} 4$ & $2.32 \mathrm{E}+03$ & $2.32 E+04$ & $5.18 E+04$ & $3.38 E+04$ \\
\hline$Z n+2$ & $N / A$ & $N / A$ & N/A & N/A & $\mathrm{N} / \mathrm{A}$ & $\mathrm{N} / \mathrm{A}$ & N/A & N/A \\
\hline $\mathrm{ZrO} 2: 2 \mathrm{H} 2 \mathrm{O}$ & & $4.13 E+03$ & & & & & & \\
\hline Total & $6.20 \mathrm{E}+03$ & $8.96 \mathrm{E}+04$ & $2.35 E+04$ & $8.52 E+04$ & $8.63 E+03$ & $2.98 \mathrm{E}+04$ & $1.10 \mathrm{E}+05$ & $7.48 \mathrm{E}+04$ \\
\hline
\end{tabular}


Table A-1. Chemical Inventory Estimates for Single-Shell Tanks

\begin{tabular}{|c|c|c|c|c|c|c|c|c|}
\hline $\begin{array}{c}\text { Insoluble } \\
\text { (kg) }\end{array}$ & U-104 & U-105 & U-106 & U-107 & U-108 & U-109 & $\mathrm{U}-110$ & U-111 \\
\hline \multicolumn{9}{|l|}{$\mathrm{Ag}+$} \\
\hline$A \mathrm{I}+3$ & & $1.09 E+02$ & $6.28 \mathrm{E}+01$ & $2.48 E+04$ & $1.68 \mathrm{E}+04$ & $1.56 \mathrm{E}+04$ & $1.51 \mathrm{E}+04$ & $8.51 E+03$ \\
\hline$A s+5$ & N/A & N/A & N/A & N/A & $\mathrm{N} / \mathrm{A}$ & $N / A$ & N/A & N/A \\
\hline$B+3$ & N/A & $N / A$ & N/A & N/A & N/A & $\mathrm{N} / \mathrm{A}$ & N/A & N/A \\
\hline $\mathrm{Ba}+2$ & & 4.17E-01 & $6.40 E-01$ & $6.88 \mathrm{E}-01$ & 8.90 E-01 & 5.07E-01 & $6.77 \mathrm{E}-01$ & $1.57 \mathrm{E}+00$ \\
\hline $\mathrm{Be}+2$ & N/A & $N / A$ & $N / A$ & N/A & N/A & N/A & N/A & N/A \\
\hline $\mathrm{Bi}+3$ & & 7.77E-01 & 2.87E-01 & $7.33 \mathrm{E}-01$ & $8.21 \mathrm{E}-01$ & 7.87E-01 & $2.82 \mathrm{E}+03$ & $2.32 \mathrm{E}+02$ \\
\hline $\mathrm{Ca}+2$ & $1.95 E+02$ & $1.50 E+02$ & $1.43 E+02$ & $6.43 E+02$ & $4.55 E+02$ & $4.82 E+02$ & $5.97 E+02$ & $1.54 E+02$ \\
\hline Cancrinite & & $1.51 E+04$ & $5.37 E+03$ & $1.47 E+04$ & $1.21 \mathrm{E}+04$ & $1.47 E+04$ & $1.47 E+04$ & $1.01 E+04$ \\
\hline $\mathrm{Cd}+2$ & N/A & N/A & N/A & N/A & $N / A$ & $N / A$ & N/A & $N / A$ \\
\hline $\mathrm{C}_{\theta}+3$ & & & & $1.05 E+03$ & $3.7 \& \mathrm{E}-\mathrm{O} 2$ & $9.61 E+00$ & $6.10 E-01$ & $2.82 E+02$ \\
\hline $\mathrm{Cl}-$ & $8.26 \mathrm{E}-01$ & $2.48 \mathrm{E}+02$ & $1.09 E+02$ & $2.29 E+02$ & $2.79 E+02$ & $2.67 E+02$ & $2.01 E+01$ & $2.17 E+02$ \\
\hline $\mathrm{Co}+3$ & N/A & N/A & N/A & N/A & N/A & N/A & N/A & N/A \\
\hline $\cos -2$ & $4.34 \mathrm{E}+02$ & $5.04 \mathrm{E}+02$ & $4.83 E+02$ & $6.36 \mathrm{E}+02$ & $4.38 E+02$ & $6.36 E+02$ & $1.72 \mathrm{E}+02$ & $2.42 \mathrm{E}+02$ \\
\hline $\mathrm{Cr}+3$ & $9.59 E+00$ & $2.69 E+01$ & $1.41 E+01$ & $3.23 E+01$ & $2.53 E+01$ & $2.88 E+01$ & $8.64 E+01$ & $3.60 \mathrm{E}+\mathrm{O2}$ \\
\hline Cst & & $2.74 \mathrm{E}-04$ & & $5.02 \mathrm{E}-01$ & $3.94 \mathrm{E}-04$ & $1.22 \mathrm{E}-01$ & $6.35 \mathrm{E}-03$ & $1.33 \mathrm{E}-07$ \\
\hline $\mathrm{Cu}+2$ & N/A & $\mathrm{N} / \mathrm{A}$ & $N / A$ & $N / A$ & $N / A$ & $\mathrm{~N} / \mathrm{A}$ & N/A & N/A \\
\hline F- & & $3.61 E+01$ & $1.33 \mathrm{E}+01$ & $3.40 E+01$ & $3.81 \mathrm{E}+01$ & $3.65 E+01$ & $3.00 \mathrm{E}+02$ & $5.68 \mathrm{E}+01$ \\
\hline $\mathrm{Fe}+3$ & $6.48 E+02$ & $5.02 \mathrm{E}+02$ & $4.23 E+02$ & $1.56 \mathrm{E}+03$ & $1.79 \equiv+03$ & $1.04 \mathrm{E}+03$ & $2.62 \mathrm{E}+03$ & $5.42 \mathrm{E}+02$ \\
\hline $\mathrm{Hg}+2$ & $\mathrm{~N} / \mathrm{A}$ & N/A & $N / A$ & $N / A$ & $N / A$ & $\mathrm{~N} / \mathrm{A}$ & N/A & N/A \\
\hline $\mathrm{K}+$ & & $1.15 E+01$ & $6.44 E+00$ & $1.19 E+01$ & $1.57 \equiv+01$ & $1.50 E+01$ & & $1.19 E+01$ \\
\hline \multicolumn{9}{|l|}{$\mathrm{La}+3$} \\
\hline $\mathrm{Mg}+2$ & $\mathrm{~N} / \mathrm{A}$ & $N / A$ & $\mathrm{~N} / \mathrm{A}$ & N/A & N/A & N/A & N/A & N/A \\
\hline $\mathrm{Mn}+4$ & & $1.49 E+03$ & $8.35 E+02$ & $1.54 E+03$ & $2.04 i+03$ & $1.94 \mathrm{E}+03$ & & $1.54 E+03$ \\
\hline $\mathrm{Mo}+6$ & N/A & N/A & $\mathrm{N} / \mathrm{A}$ & N/A & $N / A$ & N/A & $N / A$ & N/A \\
\hline $\mathrm{Na}+$ & $1.31 E+03$ & $3.67 E+03$ & $2.37 E+03$ & 3.77E + O3 & $3.501=+03$ & $4.05 E+03$ & $4.84 E+03$ & $2.87 E+03$ \\
\hline $\mathrm{Ni}+3$ & $2.50 \mathrm{E}+\mathrm{O} 2$ & $1.81 \mathrm{E}+02$ & $1.69 \mathrm{E}+02$ & $7.27 \mathrm{E}+02$ & $6.561=02$ & $5.75 E+02$ & $6.40 E+02$ & $1.78 \mathrm{E}+02$ \\
\hline NO2- & & $1.43 E+03$ & $5.62 \mathrm{E}+02$ & $1.44 E+03$ & $1.57 \mid \equiv+03$ & $1.55 E+03$ & $5.17 E+01$ & $1.23 E+03$ \\
\hline NO3- & $2.92 \mathrm{E}+01$ & $9.82 \mathrm{E}+03$ & $6.33 E+03$ & $6.77 \mathrm{E}+03$ & $1.17 \equiv+04$ & $1.11 E+04$ & $5.09 E+02$ & $7.85 E+03$ \\
\hline $\mathrm{OH}$ & $4.11 \mathrm{E}+03$ & $3.65 E+03$ & $1.79 E+03$ & $5.26 \mathrm{E}+04$ & $3.341 \equiv+0.4$ & $3.19 E+04$ & $1.32 E+04$ & $1.43 \mathrm{E}+04$ \\
\hline $\mathrm{Pb}+4$ & & $1.72 \mathrm{E}-03$ & $9.67 E-04$ & $1.78 \mathrm{E}-03$ & $2.36 \mathrm{E}-03$ & $2.25 E-03$ & & $1.78 \mathrm{E}-03$ \\
\hline PO4-3 & $4.94 \mathrm{E}+03$ & $1.35 E+03$ & $3.27 \mathrm{E}+03$ & $4.82 E+03$ & $5.18 E+02$ & $3.10 \mathrm{E}+03$ & $4.34 E+04$ & $4.19 E+03$ \\
\hline $\mathrm{Rb}+$ & N/A & $N / A$ & $N / A$ & $N / A$ & N/A & N/A & N/A & N/A \\
\hline$R \theta+7$ & N/A & N/A & $N / A$ & $N / A$ & $N / A$ & $\mathrm{~N} / \mathrm{A}$ & N/A & $N / A$ \\
\hline $\mathrm{Rh}+\mathbf{3}$ & N/A & $\mathrm{N} / \mathrm{A}$ & $\mathrm{N} / \mathrm{A}$ & N/A & N/A & N/A & N/A & $\mathrm{N} / \mathrm{A}$ \\
\hline $\mathrm{Ru}+3$ & N/A & N/A & N/A & $\mathrm{N} / \mathrm{A}$ & NIA & N/A & N/A & N/A \\
\hline$S e+6$ & N/A & N/A & N/A & N/A & $N / A$ & N/A & N/A & $N / A$ \\
\hline$S i+4$ & $1.36 \mathrm{E}+00$ & & & & & & & \\
\hline so4-2 & $1.00 E+02$ & $1.80 E+02$ & $1.28 \mathrm{E}+02$ & $1.85 E+02$ & $1.40 \mathrm{O}=02$ & $1.86 \mathrm{E}+02$ & $4.85 E+01$ & $9.63 E+01$ \\
\hline $\mathrm{Sr}+2$ & & $1.65 \mathrm{E}-05$ & & $3.30 E+01$ & $2.141 \equiv+01$ & $2.06 \mathrm{E}+01$ & 1.57E + 00 & 2.01E-01 \\
\hline $\mathrm{Te}+6$ & N/A & N/A & N/A & N/A & $N / A$ & N/A & $\mathrm{N} / \mathrm{A}$ & $N / A$ \\
\hline$T h+4$ & N/A & N/A & N/A & N/A & $N / A$ & $N / A$ & N/A & $\mathrm{N} / \mathrm{A}$ \\
\hline$T I+4$ & N/A & N/A & N/A & N/A & $N / A$ & N/A & N/A & N/A \\
\hline TOC & & $8.78 \mathrm{E}+01$ & $3.30 \mathrm{E}+01$ & $8.68 \mathrm{E}+01$ & $9.761 \pm+01$ & $9.35 \mathrm{E}+01$ & & $7.74 E+01$ \\
\hline $\mathrm{UO}_{2}+2$ & $4.23 E+04$ & $3.60 E+04$ & $2.75 E+04$ & $4.76 E+04$ & $9.971 \equiv+0.3$ & $3.01 E+04$ & $9.83 E+03$ & $1.82 E+02$ \\
\hline $2 n+2$ & N/A & N/A & N/A & N/A & $N / A$ & N/A & N/A & $N / A$ \\
\hline $\mathrm{ZrO} 2: 2 \mathrm{H} 2 \mathrm{O}$ & & & & & & & $3.83 \mathrm{E}+03$ & $3.81 E+02$ \\
\hline Total & $5.44 \mathrm{E}+04$ & $7.45 E+04$ & $4.97 E+04$ & $1.63 E+05$ & $9.561 \equiv+04$ & $1.17 \mathrm{E}+05$ & $1.13 \mathrm{E}+05$ & $5.36 \mathrm{E}+04$ \\
\hline
\end{tabular}


WHC-SD-WM-TI-784

Revision 0

Table A-1. Chemical Inventory Estimates for Single-Shell Tanks

\begin{tabular}{|c|c|c|c|c|c|c|c|c|}
\hline $\begin{array}{c}\text { Insoluble } \\
\text { (kg) }\end{array}$ & $U-112$ & $U-201$ & $\mathrm{U}-202$ & U-203 & U-204 & Total & $\begin{array}{l}\text { SALTWELL } \\
\text { LIO. PUMPED }\end{array}$ & NET TOTAL \\
\hline$A g+$ & & & & & & $2.80 \mathrm{E}-04$ & & $2.80 \mathrm{E}-04$ \\
\hline$A \mathrm{I}+3$ & $1.20 E+04$ & $2.71 E+03$ & $2.71 E+03$ & $1.35 E+03$ & $1.35 E+03$ & $2.20 E+06$ & & $2.20 E+06$ \\
\hline$A s+5$ & N/A & N/A & N/A & $\mathrm{N} / \mathrm{A}$ & $\mathrm{N} / \mathrm{A}$ & & & \\
\hline$B+3$ & N/A & N/A & N/A & N/A & N/A & & & \\
\hline $\mathrm{Ba}+2$ & $1.22 \mathrm{E}+00$ & $1.74 \mathrm{E}-01$ & $1.84 \mathrm{E}-01$ & $1.62 E-01$ & $4.231 \equiv-02$ & $5.89 E+02$ & & $5.89 E+02$ \\
\hline$B o+2$ & N/A & N/A & N/A & N/A & $N / A$ & & & \\
\hline$B i+3$ & $9.15 E+02$ & & & & & $2.52 E+05$ & & $2.52 E+05$ \\
\hline $\mathrm{Ca}+2$ & $2.28 E+02$ & $2.63 E+01$ & $2.63 E+01$ & $1.32 E+01$ & $1.32 E+01$ & $1.22 E+05$ & & $1.22 E+05$ \\
\hline Cancrinite & $5.20 E+03$ & $5.14 E+02$ & $5.14 E+02$ & $2.57 E+02$ & & $2.70 E+06$ & & $2.70 E+06$ \\
\hline$C d+2$ & N/A & N/A & N/A & N/A & $N / A$ & & & \\
\hline $\mathrm{Ce}_{\theta}+3$ & $3.48 \mathrm{E}+03$ & $1.83 E+02$ & $1.82 \mathrm{E}+\mathrm{O} 2$ & $1.60 E+02$ & $3.67 E+01$ & $2.32 E+05$ & & $2.32 E+05$ \\
\hline $\mathrm{Cl}-$ & $5.54 E+\infty 0$ & 3.13E-01 & $3.13 \mathrm{E}-01$ & $1.56 \mathrm{E}-01$ & $1.561:-01$ & $7.63 E+03$ & & $7.63 E+03$ \\
\hline $\mathrm{Co}+3$ & N/A & N/A & N/A & N/A & $N / A$ & & & \\
\hline co3-2 & $3.07 E+01$ & $3.55 E+00$ & $3.55 E+\infty$ & $1.77 E+00$ & $1.77 E+00$ & $3.86 \mathrm{E}+04$ & & $3.86 E+04$ \\
\hline $\mathrm{Cr}+3$ & $2.00 E+02$ & $1.53 E+00$ & $1.53 E+00$ & $7.64 \mathrm{E}-01$ & $7.64 \mathrm{E}=-01$ & $1.21 E+05$ & & $1.21 E+05$ \\
\hline Cst & $5.78 \mathrm{E}-01$ & $9.12 E-02$ & $9.58 \mathrm{E}-02$ & $8.44 \mathrm{E} \cdot 02$ & $2.24 \mid=-02$ & $1.75 E+02$ & & $1.75 E+02$ \\
\hline $\mathrm{Cu+2}$ & N/A & N/A & N/A & N/A & $N / A$ & & & \\
\hline F. & $7.01 E+01$ & & & & & $2.62 E+04$ & & $2.62 E+04$ \\
\hline $\mathrm{Fe}+3$ & $1.14 E+03$ & $9.57 E+01$ & $9.57 E+01$ & $4.79 E+01$ & $4.79 E+01$ & $6.07 E+05$ & & $6.07 \mathrm{E}+05$ \\
\hline $\mathrm{Hg}+2$ & N/A & N/A & N/A & N/A & $N / 4$ & & & \\
\hline$K+$ & & & & & & $2.43 E+03$ & & $2.43 E+03$ \\
\hline $\mathrm{La+3}$ & & & & & & $1.86 E+03$ & & $1.86 \mathrm{E}+03$ \\
\hline$M g+2$ & N/A & N/A & N/A & N/A & N/A & & & \\
\hline$M n+4$ & & & & & & $1.18 E+05$ & & $1.18 E+05$ \\
\hline$M o+6$ & N/A & N/A & N/A & N/A & $N / A$ & & & \\
\hline $\mathrm{Na}+$ & $1.36 \mathrm{E}+03$ & $9.18 E+01$ & $9.18 E+01$ & $4.59 \mathrm{E}+01$ & $4.59 \mathrm{E}+01$ & $5.42 E+05$ & & $5.42 E+05$ \\
\hline $\mathrm{Ni}+3$ & $2.89 \mathrm{E}+02$ & $3.59 E+01$ & $3.59 \mathrm{E}+01$ & $1.79 E+01$ & $1.79 E+01$ & $1.99 E+05$ & & $1.99 \mathrm{E}+05$ \\
\hline NO2- & $2.18 E+01$ & $9.43 E+\infty 0$ & $9.43 E+\infty 0$ & $4.72 E+00$ & $4.72 \mathrm{E}+00$ & $5.34 E+04$ & & $5.34 E+04$ \\
\hline NO3- & $1.74 \mathrm{E}+02$ & $1.65 E+01$ & $1.65 \mathrm{E}+01$ & $8.27 E+00$ & $8.27 E+\infty 0$ & $9.42 E+05$ & & $9.42 E+05$ \\
\hline $\mathrm{OH}-$ & $2.04 E+04$ & $5.46 \mathrm{E}+03$ & $5.46 \mathrm{E}+03$ & $2.76 \mathrm{E}+03$ & $2.66 \mathrm{E}+03$ & $4.55 E+06$ & & $4.55 E+06$ \\
\hline $\mathrm{Pb}+4$ & & & & & & $2.50 E+04$ & & $2.50 E+04$ \\
\hline PO4-3 & $1.17 E+04$ & & & & & $1.83 E+06$ & & $1.83 E+06$ \\
\hline $\mathrm{Rb}+$ & N/A & $N / A$ & N/A & N/A & $N / 4$ & & & \\
\hline $\mathrm{Re}+7$ & $\mathrm{~N} / \mathrm{A}$ & N/A & $N / A$ & $N / A$ & $N / A$ & & & \\
\hline $\mathrm{Rh}+3$ & N/A & N/A & N/A & N/A & N/A & & & \\
\hline $\mathrm{Ru}+3$ & $\mathrm{~N} / \mathrm{A}$ & N/A & N/A & N/A & $N / 4$ & & & \\
\hline$S_{\theta+6}$ & $\mathrm{~N} / \mathrm{A}$ & N/A & N/A & $N / A$ & $N / 4$ & & & \\
\hline$S i+4$ & & & & & & $1.41 E+04$ & & $1.41 E+04$ \\
\hline SO4-2 & $4.16 E+00$ & $3.67 \mathrm{E}-02$ & $3.67 \mathrm{E}-02$ & $1.84 \mathrm{E}-02$ & $1.84:-02$ & $2.19 E+04$ & & $2.19 E+04$ \\
\hline$S r+2$ & $5.16 E+00$ & $3.42 E+\infty 0$ & $3.42 E+00$ & $1.71 E+00$ & $1.71 E+00$ & $3.59 \mathrm{E}+04$ & & $3.59 E+04$ \\
\hline $\mathrm{Te}_{\theta}+6$ & N/A & N/A & N/A & N/A & $N / A$ & & & \\
\hline $\mathrm{Th}+4$ & N/A & N/A & N/A & $N / A$ & $N / 4$ & & & \\
\hline$T I+4$ & N/A & N/A & N/A & $N / A$ & $N / 4$ & & & \\
\hline TOC & & & & & & $1.51 E+04$ & & $1.51 E+04$ \\
\hline $002+2$ & $1.25 E+03$ & $7.60 \mathrm{E}+02$ & $7.60 E+02$ & $3.80 E+02$ & $5.91 \mathrm{E}+00$ & $1.50 E+06$ & & $1.50 E+06$ \\
\hline $2 n+2$ & N/A & N/A & N/A & N/A & $N / A$ & & & \\
\hline $\mathrm{ZrO} 2: 2 \mathrm{H} 2 \mathrm{O}$ & $1.02 E+03$ & & & & & $6.61 E+05$ & & $6.61 \mathrm{E}+05$ \\
\hline Total & $5.96 \mathrm{E}+04$ & $9.91 E+03$ & $9.91 E+03$ & $5.05 E+03$ & $4.20 E+03$ & $1.68 E+07$ & & $1.68 E+07$ \\
\hline
\end{tabular}


Table A-2. Radionuclide Inventory Estimates for Single-Shell Tanks

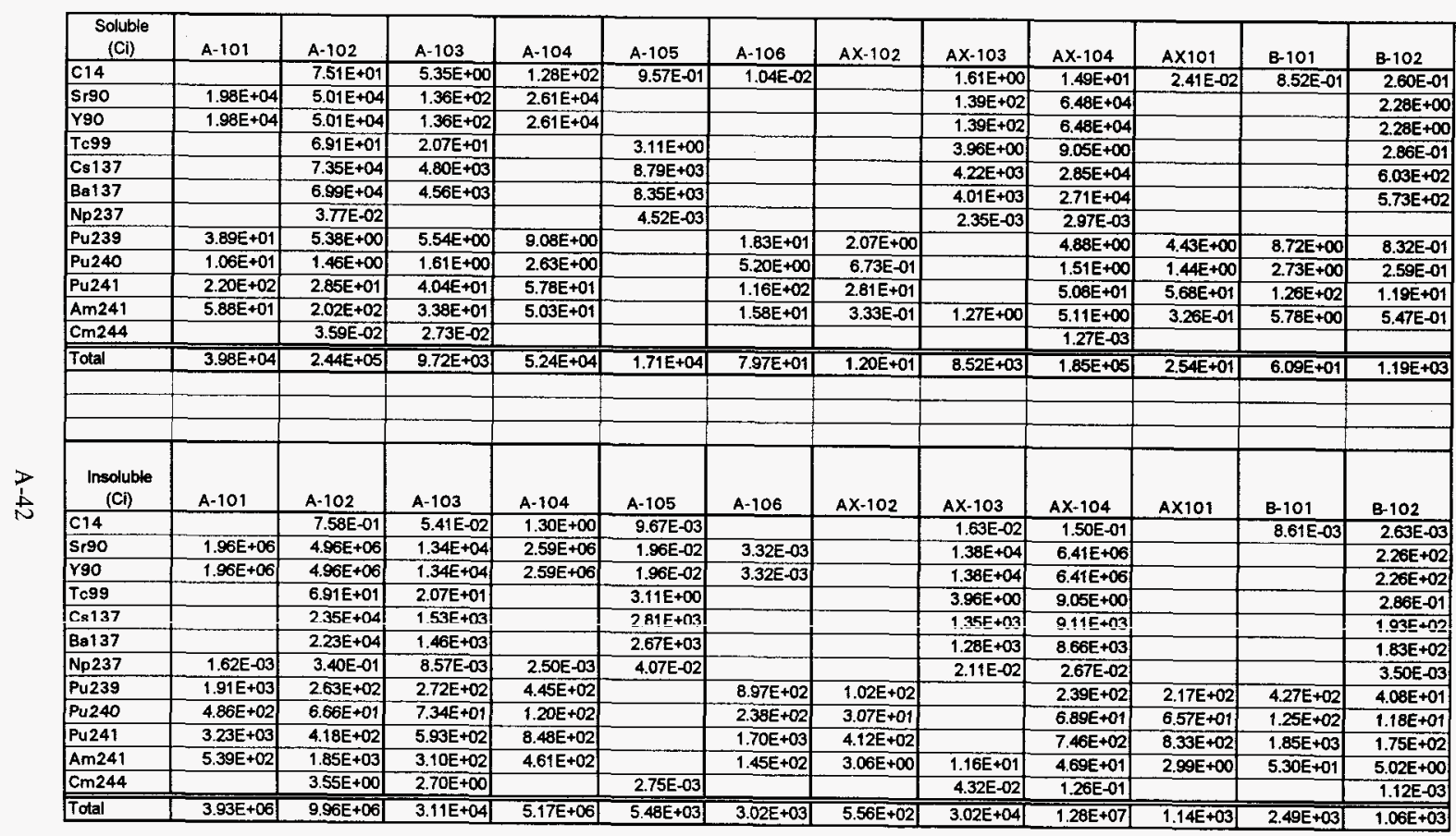


Table A-2. Radionuclide Inventory Estimates for Single-Shell Tanks

\begin{tabular}{|c|c|c|c|c|c|c|c|c|c|c|c|c|}
\hline $\begin{array}{l}\text { Soluble } \\
\text { (C) }\end{array}$ & B-103 & B-104 & B-105 & B-106 & B- 107 & B-108 & B-109 & B-110 & B-111 & B-112 & B-201 & B-202 \\
\hline $\mathrm{C} 14$ & $6.20 \mathrm{E}-01$ & $8.10 \mathrm{E}-02$ & $2.02 E+00$ & $8.49 E-02$ & $2.41 E+00$ & $1.81 \mathrm{E}+00$ & $1.27 E+00$ & $2.14 E+01$ & $4.52 E+02$ & $1.40 \mathrm{E}+01$ & & \\
\hline Sr9o & $6.84 \mathrm{E}+01$ & $5.64 E+01$ & $8,47 \mathrm{E}+00$ & $9.11 E+00$ & $1.67 \mathrm{E}+02$ & $2.99 \mathrm{E}+02$ & $3.89 \mathrm{E}+01$ & $4.36 \mathrm{E}+02$ & & $9.40 \mathrm{E}+02$ & & \\
\hline Y90 & $6.84 E+01$ & $5.64 E+01$ & $8.47 \mathrm{E}+00$ & $1.11 \mathrm{E}+00$ & $1.67 E+02$ & $2.99 \mathrm{E}+02$ & $3.89 E+01$ & $4.36 \mathrm{E}+02$ & & $9.40 E+02$ & & \\
\hline Tc99 & $1.59 \mathrm{E}+00$ & $5.57 \mathrm{E}-01$ & $4.38 E+00$ & 2.07E-01 & $3.99 \bar{E}+00$ & $8.36 E+00$ & $2.89 E+00$ & $9.27 \mathrm{E}+01$ & $9.43 E+02$ & $6.37 \mathrm{E}+01$ & & \\
\hline Cs137 & $2.44 E+03$ & $1.14 E+03$ & $9.49 E+03$ & $4.29 E+02$ & $1.11 \mathrm{E}+04$ & $7.77 \mathrm{E}+03$ & $3.13 E+03$ & $1.56 \mathrm{E}+04$ & $1.39 E+05$ & $5.71 E+04$ & & \\
\hline Ba137 & $2.31 E+03$ & $1.08 \mathrm{E}+03$ & $9.01 E+03$ & $4.08 E+02$ & $1.06 E+04$ & $7.38 \mathrm{E}+03$ & $2.97 E+03$ & $1.48 E+04$ & $1.32 E+05$ & $5.42 \mathrm{E}+04$ & & \\
\hline Np237 & $1.89 E-03$ & & 5.47E-03 & & $6.86 \mathrm{E}-03$ & 9.19E-03 & $3.23 E-03$ & $3.03 E-03$ & $1.42 E+\infty$ & $6.86 \mathrm{E}-02$ & & \\
\hline Pu239 & $2.85 E+00$ & $1.69 E+00$ & $1.69 E-01$ & $1.88 \mathrm{E}-02$ & 6.67E-01 & $6.67 \mathrm{E}-02$ & $7.40 \mathrm{E}-03$ & $3.91 E+\infty$ & $5.38 \mathrm{E}-01$ & $5.59 \mathrm{E}-02$ & & $3.11 \mathrm{E}+\infty$ \\
\hline Pu240 & $8.83 \mathrm{E}-01$ & $1.86 \mathrm{E}-01$ & $1.86 \mathrm{E}-02$ & $2.07 \mathrm{E}-03$ & 6.02E-02 & $6.02 \mathrm{E}-03$ & & $8.36 E-01$ & $1.07 \mathrm{E}-01$ & $1.10 \mathrm{E}-02$ & & 9.25E-01 \\
\hline \begin{tabular}{|l|} 
Pu241 \\
\end{tabular} & $2.31 \mathrm{E}+04$ & $8.41 \mathrm{E}-01$ & $8.41 E-02$ & $9.32 \mathrm{E}-03$ & $1.97 \mathrm{E}-01$ & $1.97 \mathrm{E}-02$ & 2.19E-03 & $2.68 \mathrm{E}+01$ & $1.04 E+\infty$ & $1.05 \mathrm{E}-01$ & & $2.03 E+01$ \\
\hline Am241 & $3.61 E+\infty$ & $2.56 \mathrm{E}-01$ & $2.62 E-02$ & $1.48 \mathrm{E}-02$ & $7.33 \mathrm{E}-02$ & 3.28E-01 & $1.21 E-01$ & $8.20 E+01$ & $2.48 \mathrm{E}-01$ & $2,44 E+00$ & & $2.26 E+\infty 0$ \\
\hline $\mathrm{Cm} 244$ & & & & & & & & $1.02 \mathrm{E}-01$ & $7.95 E-03$ & 5.24E-03 & & \\
\hline Total & $4.90 \mathrm{E}+03$ & $2.34 \mathrm{E}+03$ & $1.85 E+04$ & $8.40 E+02$ & $2.20 E+04$ & $1.58 \mathrm{E}+04$ & $6.19 \mathrm{E}+03$ & $3.14 \mathrm{E}+04$ & $2.72 E+05$ & $1.13 \mathrm{E}+05$ & & $1.26 \mathrm{E}+01$ \\
\hline & & & & & & & & & & & & \\
\hline & & & & & & & & & & & & \\
\hline & & & & & & & & & & & & \\
\hline $\begin{array}{c}\text { Insoluble } \\
\text { (Ci) }\end{array}$ & B-103 & B-104 & B-105 & B-106 & B-107 & B-108 & B-109 & B-110 & B-111 & B-112 & B-201 & B-202 \\
\hline C14 & $6.26 \mathrm{E}-03$ & & $2.04 E-02$ & & 2.44E-02 & $4.83 \mathrm{E}=02$ & $1.29 \mathrm{E}-02$ & $2.16 \mathrm{E}-01$ & $4.57 E+\infty$ & $1.41 \mathrm{E}-01$ & & \\
\hline Sr90 & $6.78 \mathrm{E}+03$ & $5.58 \mathrm{E}+03$ & $8,38 E+02$ & $1.10 \mathrm{E}+02$ & $1.65 E+04$ & $2.96 \mathrm{E}+04$ & $3.85 E+03$ & $4.32 \mathrm{E}+04$ & & $9.30 E+04$ & & \\
\hline Y90 & $6.78 E+03$ & $5.58 E+03$ & $8.38 E+02$ & $1.10 E+02$ & $1.65 E+04$ & $2.96 \mathrm{E}+04$ & $3.85 \mathrm{E}+03$ & $4.32 E+04$ & & $9.30 \mathrm{E}+04$ & & \\
\hline Tc99 & $1.59 E+00$ & 5.57E-01 & $4.38 \mathrm{E}+00$ & 2.07E-01 & $3.99 E+\infty$ & $8.36 \mathrm{E}+00$ & $2.69 E+00$ & $9.27 \mathrm{E}+01$ & $9.43 E+02$ & $6.37 E+01$ & & \\
\hline Cs137 & $7.78 E+02$ & $3.64 E+02$ & $3.03 E+03$ & $1.37 \dot{E}+02$ & $355 E+03$ & $2.48 \mathrm{E}+03$ & $100 F+03$ & $497 F+03$ & $444 F+04$ & $183 F+04$ & & \\
\hline $\operatorname{Ba137}$ & $7.39 \mathrm{E}+02$ & $3.46 E+02$ & $2.88 \mathrm{E}+03$ & $1.30 \mathrm{E}+02$ & $3.37 E+03$ & $2.36 \mathrm{E}+03$ & $9.50 \mathrm{E}+02$ & $4.72 E+03$ & $4.22 \mathrm{E}+04$ & $1.73 \mathrm{E}+04$ & & \\
\hline Np237 & $1.71 \mathrm{E}-02$ & $6.30 \mathrm{E}-03$ & 4.93E-02 & 2.48E-03 & $6.18 E-02$ & 8.27E-02 & $2.90 E-02$ & $2.73 E-02$ & $1.27 E+01$ & $6.18 \mathrm{E}-01$ & & \\
\hline Pu239 & $1.40 \mathrm{E}+02$ & $8.30 \mathrm{E}+01$ & $8.30 E+C 0$ & $9.22 \mathrm{E}-01$ & $3.27 E+01$ & $3.27 \mathrm{E}+00$ & 3.63E-01 & $1.92 \mathrm{E}+02$ & $2.63 E+01$ & $2.74 E+00$ & & $1.53 \mathrm{E}+02$ \\
\hline Pu240 & $4.03 E+01$ & $8.51 \mathrm{E}+00$ & $8.51 E-01$ & $9.45 E-02$ & $2.75 \mathrm{E}+\infty$ & $275 \mathrm{E}-01$ & $3.07 \mathrm{E}-02$ & $3.82 \mathrm{E}+01$ & $4.91 \mathrm{E}+00$ & 5.04E-01 & & $4.22 \mathrm{E}+01$ \\
\hline Pu241 & $3.39 \mathrm{E}+02$ & $1.23 \mathrm{E}+01$ & $1.23 \mathrm{E}+00$ & 1.37E-01 & $2.89 \mathrm{E}+\infty 0$ & $2.89 \mathrm{E}-01$ & $3.21 \mathrm{E}-02$ & $3.93 E+02$ & $1.52 E+01$ & $1.54 E+00$ & & $2.98 E+02$ \\
\hline Am241 & $3.31 \mathrm{E}+01$ & $2.35 \mathrm{E}+00$ & $2,40 \mathrm{E}-01$ & $1,35 \mathrm{E}-01$ & $6.72 \mathrm{E}-01$ & $3.01 E+00$ & $1.11 \mathrm{E}+00$ & $7.52 \mathrm{E}+02$ & $2.27 \mathrm{E}+00$ & $2.23 E+01$ & & 2.07E+01 \\
\hline Cm244 & $1.10 \mathrm{E}-02$ & & & & $3.16 \mathrm{E}-03$ & $6.88 E-02$ & $2.18 \mathrm{E}-02$ & $1.01 \mathrm{E}+01$ & 7.87E-01 & $5.18 \mathrm{E}-01$ & & \\
\hline Total & $1.56 \mathrm{E}+04$ & $1.20 \mathrm{E}+04$ & $7.60 \mathrm{E}+03$ & $4.89 \mathrm{E}+02$ & $4.01 \mathrm{E}+04$ & $6.41 \mathrm{E}+04$ & $9.65 \mathrm{E}+03$ & $9.76 \mathrm{E}+04$ & $8.75 E+04$ & $2.22 \mathrm{E}+05$ & & $5.20 \mathrm{E}+02$ \\
\hline
\end{tabular}


Table A-2. Radionuclide Inventory Estimates for Single-Shell Tanks

\begin{tabular}{|c|c|c|c|c|c|c|c|c|c|c|c|c|}
\hline $\begin{array}{c}\text { Soluble } \\
\text { (Ci) }\end{array}$ & B-203 & B-204 & BX-101 & $\mathrm{BX}-102$ & $\mathrm{BX}-103$ & $3 x-104$ & $\mathrm{BX}-105$ & $\mathrm{BX}-106$ & $\mathrm{BX}-107$ & $B X-108$ & $B X-109$ & $B X-110$ \\
\hline $\mathrm{C14}$ & & & $4.25 E+00$ & $1.03 E+02$ & $1.61 E+01$ & $2.11 E+02$ & $1.43 E+02$ & $3.92 \mathrm{E}+02$ & $4.55 \mathrm{E}-02$ & $1.47 \mathrm{E}-02$ & $1.24 E+00$ & $2.21 E+01$ \\
\hline Sr9o & & & & $8.90 \mathrm{E}+02$ & $1.74 \mathrm{E}+03$ & $1.31 \mathrm{E}+02$ & $2.81 E+04$ & $1.15 E+04$ & $3.10 \mathrm{E}+02$ & $3.39 \mathrm{E}+01$ & $2.74 E+03$ & $1.53 E+03$ \\
\hline Y90 & & & & $8.90 E+02$ & $1.74 \mathrm{E}+03$ & $1.31 \mathrm{E}+02$ & $2.81 E+04$ & $1.15 E+04$ & $3.10 \mathrm{E}+02$ & $3.39 E+01$ & $2.74 E+03$ & $1.53 E+03$ \\
\hline To99 & & & & $1.99 \mathrm{E}+02$ & & $1.02 \mathrm{E}+03$ & $4.79 \mathrm{E}+02$ & $7.44 E+02$ & $1.64 E+00$ & $3.81 E-02$ & $1.40 \mathrm{E}+00$ & $1.01 E+02$ \\
\hline Cs 137 & & & & $6.43 E+04$ & & $1.54 E+05$ & $3.66 \mathrm{E}+04$ & $1.37 \mathrm{E}+06$ & $3.36 E+03$ & $1.10 E * 02$ & $3.80 \mathrm{E}+03$ & $9.05 E+04$ \\
\hline Ba137 & & & & $6.10 \mathrm{E}+04$ & & $1.46 E+05$ & $3.48 E+04$ & 1.30E+06 & $3.20 \mathrm{E}+03$ & $1.05 E+02$ & $3.61 \mathrm{E}+03$ & $8.59 E+04$ \\
\hline Np237 & & & & $1.05 E-01$ & & $1.13 E+\infty$ & $1.22 \mathrm{E}-01$ & 4.89E-01 & 2.11E-03 & & $2.87 E-03$ & 1.09E-01 \\
\hline Pu239 & $1.05 \mathrm{E}-02$ & $1.05 E-01$ & $7.64 E+00$ & 4.31E-01 & & $1.00 \mathrm{E}+00$ & & & $7.40 E-01$ & $7.40 E-02$ & 9.29E-01 & 2.85E-01 \\
\hline Pu240 & $2.69 E-03$ & $2.69 E-02$ & $2.46 E+\infty$ & 1.57E-01 & & $3.10 \mathrm{E}-01$ & & & $8.11 \mathrm{E}-02$ & 8.11E-03 & 1.34E-01 & $4.26 \mathrm{E}-02$ \\
\hline Pu241 & $3.08 E-02$ & $3.08 E-01$ & $1.01 E+02$ & $7.04 E+\infty 0$ & & $7.95 E+\infty$ & & & $3.83 E-01$ & $3.83 E-02$ & 8.41E-01 & $2.60 \mathrm{E}-01$ \\
\hline Am 241 & 6.71E-03 & 6.71E-02 & $6.14 E+00$ & $4.40 \mathrm{E}+01$ & & $1.12 \mathrm{E}+00$ & $2.26 E+02$ & $1.59 E+02$ & $1.82 \mathrm{E}-01$ & $1.82 E-02$ & $1.57 \mathrm{E}+00$ & $3.92 E+00$ \\
\hline $\mathrm{Crn} 244$ & & & & $2.47 \mathrm{E}-02$ & & 1.08E-02 & $1.03 E-01$ & $8.58 \mathrm{E}-02$ & & & & $8.29 \mathrm{E}-03$ \\
\hline Total & $2.93 \mathrm{E}-02$ & 2.93E-01 & $5.21 \mathrm{E}+01$ & $1.27 \mathrm{E}+05$ & $3.49 \mathrm{E}+03$ & $3.01 \mathrm{E}+05$ & $1.28 \mathrm{E}+05$ & $2.70 E+06$ & $7.18 \mathrm{E}+03$ & $2.83 E+02$ & $1.29 \mathrm{E}+04$ & $1.80 \mathrm{E}+05$ \\
\hline & & & & & & & & & & & & \\
\hline & & & & & & & & & & & & \\
\hline & & & & & & & & & & & & \\
\hline $\begin{array}{c}\text { Insoluble } \\
\text { (Ci) }\end{array}$ & B-203 & B-204 & BX-101 & BX-102 & BX-103 & BX-104 & $B X-105$ & BX-106 & BX-107 & BX-108 & BX-109 & BX-110 \\
\hline C14 & & & $4.29 \mathrm{E}-02$ & $1.05 E+00$ & $1.63 \mathrm{E}-01$ & $2.13 E+00$ & $1.45 \mathrm{E}+00$ & $3.96 E+\infty$ & & & $1.25 E-02$ & $2.23 E-01$ \\
\hline $5 r 90$ & & & & $8.81 E+04$ & $1.72 \mathrm{E}+05$ & $1.29 \mathrm{E}+04$ & $2.78 \mathrm{E}+06$ & $1.14 \mathrm{E}+06$ & $3.07 E+04$ & $3.36 \mathrm{E}+03$ & $2.71 E+05$ & $1.51 E+05$ \\
\hline Y90 & & & & $8.81 \mathrm{E}+04$ & $1.72 \mathrm{E}+05$ & $1.29 \mathrm{E}+04$ & $2.78 \mathrm{E}+06$ & $1.14 E+06$ & $3.07 E+04$ & $3.36 \mathrm{E}+03$ & $2.71 E+05$ & $1.51 E+05$ \\
\hline To99 & & & & $1.99 \mathrm{E}+02$ & & $1.02 \mathrm{E}+03$ & $4.79 \mathrm{E}+02$ & $7.44 \mathrm{E}+02$ & $1,64 \mathrm{E}+00$ & $3.81 \mathrm{E}-02$ & $4.40 \mathrm{E}+00$ & $1.01 \mathrm{E}+02$ \\
\hline Cs137 & & & & $2.05 E+04$ & & $4.90 \mathrm{E}+04$ & $1.17 \mathrm{E}+04$ & $4.38 E+05$ & $1.07 \mathrm{E}+03$ & $3.53 E+01$ & $1.21 \mathrm{E}+03$ & $2.89 E+04$ \\
\hline Ba137 & & & & $1.95 \mathrm{E}+04$ & & $4.66 E+04$ & $1.11 E+04$ & $4.16 \mathrm{E}+\mathrm{CS}$ & $1.02 \mathrm{E}+03$ & $3.35 \mathrm{E}+01$ & $1.15 E+03$ & $2.74 E+04$ \\
\hline Np237 & & & & $9.45 E-01$ & & $1.02 \mathrm{E}+01$ & $1.10 \mathrm{E}+\infty$ & $4.40 \mathrm{E}+00$ & $1.90 \mathrm{E}-02$ & & $2.59 E-02$ & $9.78 \mathrm{E}-01$ \\
\hline Pu239 & $5.13 \mathrm{E}-01$ & $5.13 E+\infty 0$ & $3.74 E+02$ & $2.11 E+01$ & & $4.92 \mathrm{E}+01$ & & $1.08 E-03$ & $3.63 \mathrm{E}+01$ & $3.63 E+00$ & 4.55E+01 & $1.40 E+01$ \\
\hline Pu240 & 1.23E-01 & $1.23 E+\infty$ & $1.13 E+02$ & $7.16 \mathrm{E}+00$ & & $1.42 \mathrm{E}+01$ & $1.54 E-02$ & $1.54 \mathrm{E}-02$ & $3.70 \mathrm{E}+00$ & $3.70 E-01$ & $6.12 \mathrm{E}+00$ & $1.94 E+00$ \\
\hline Pu241 & 4.51E-01 & $4.51 E+\infty$ & $1.48 E+03$ & $1.03 E+02$ & & $1.17 \mathrm{E}+02$ & $1.09 \mathrm{E}-03$ & $2.13 E-03$ & $5.61 E+00$ & 5.61E-01 & 1.23E+01 & $3.81 E+00$ \\
\hline Am241 & $6.15 \mathrm{E}-02$ & $6.15 E-01$ & $5.63 E+01$ & $4.03 E+02$ & & $1.03 E+01$ & $2.08 E+03$ & $1.46 \mathrm{E}+03$ & $1.67 \mathrm{E}+00$ & $1.67 \mathrm{E}-01$ & $1.44 E+01$ & $3.60 \mathrm{E}+01$ \\
\hline $\mathrm{Cm} 244$ & & & & $2.44 E+\infty$ & & $1.07 \mathrm{E}+00$ & $1.02 \mathrm{E}+01$ & $8.50 E+\infty$ & & & & 8.21E-01 \\
\hline Total & $1.15 \mathrm{E}+00$ & $1.15 \mathrm{E}+01$ & $2.04 \mathrm{E}+03$ & $2.17 E+05$ & $3.44 E+05$ & $1.23 \mathrm{E}+05$ & $5.58 \mathrm{E}+06$ & $3.13 \mathrm{E}+06$ & $6.35 \mathrm{E}+04$ & $6.79 \mathrm{E}+03$ & $5.45 E+05$ & $3.59 \mathrm{E}+05$ \\
\hline
\end{tabular}


Table A-2. Radionuclide Inventory Estimates for Single-Shell Tanks

\begin{tabular}{|c|c|c|c|c|c|c|c|c|c|c|c|c|}
\hline $\begin{array}{l}\text { Soluble } \\
\text { (Ci) }\end{array}$ & $B X-111$ & $8 x-112$ & BY-101 & $\mathrm{BY}-102$ & BY-103 & BY-104 & BY-105 & BY-106 & BY-107 & BY-108 & BY-109 & BY-110 \\
\hline C14 & $2.28 \mathrm{E}+01$ & $5.93 E-01$ & $2.91 E+01$ & $8.44 E+\infty$ & $2.49 E+\infty$ & $3.04 E+01$ & $2.41 \mathrm{E}+01$ & $2.95 E+01$ & $1.08 E+01$ & $1.11 \mathrm{E}+01$ & $9.43 E+00$ & $2.64 E+01$ \\
\hline Sr90 & $1.52 \mathrm{E}+03$ & $3.11 E+01$ & $2.38 E+03$ & $9.76 \mathrm{E}+02$ & $4.89 \mathrm{E}+03$ & $1.99 \mathrm{E}+03$ & $1.67 \mathrm{E}+03$ & $2.54 \mathrm{E}+03$ & $1.09 \mathrm{E}+03$ & $3.53 E+02$ & $7.01 E+02$ & $2.32 E+03$ \\
\hline Y9o & $1.52 \mathrm{E}+03$ & $3.11 \mathrm{E}+01$ & $2.38 E+03$ & $9.76 \mathrm{E}+02$ & $4.89 \mathrm{E}+03$ & $1.99 E+03$ & $1.67 \mathrm{E}+03$ & $2.54 E+03$ & $1.09 \mathrm{E}+03$ & $3.53 E+02$ & $7.01 E+02$ & $2.32 \mathrm{E}+03$ \\
\hline To99 & $1.04 E+02$ & 6.27E-01 & $1.33 E+02$ & $3.86 E+01$ & $6.27 \mathrm{E}+00$ & $1,36 E+02$ & $1.06 \mathrm{E}+02$ & $1.35 E+02$ & $4.92 E+01$ & $5.95 E+01$ & $4.32 E+01$ & $1.21 E+02$ \\
\hline Cs 137 & $9.30 \mathrm{E}+04$ & $1.68 \mathrm{E}+03$ & $1.19 E+05$ & $3.46 \mathrm{E}+04$ & $5.73 E+03$ & $1.22 \mathrm{E}+05$ & $9.44 E+04$ & $1.21 E+05$ & $4.41 \mathrm{E}+04$ & $5.71 \mathrm{E}+04$ & $3.85 E+04$ & $1.08 \mathrm{E}+05$ \\
\hline$B a 137$ & $8.84 E+04$ & $1.60 \mathrm{E}+03$ & $1.13 E+05$ & $3.28 \mathrm{E}+04$ & $5.44 E+03$ & $1.15 \mathrm{E}+05$ & $8.97 \mathrm{E}+04$ & $1.15 E+05$ & $4.19 E+04$ & $5.42 E+04$ & $3.66 \mathrm{E}+04$ & $1.02 \mathrm{E}+05$ \\
\hline Np237 & $1.12 \mathrm{E}-01$ & $1.22 \mathrm{E}-03$ & 1.43E-01 & 4.15E-02 & $7.27 \mathrm{E}-03$ & 1.46E-01 & $1.14 E-01$ & 1.45E-01 & 5.30E-02 & $8.45 \mathrm{E}-02$ & $4.63 \mathrm{E}-02$ & $1.30 \mathrm{E}-01$ \\
\hline Pu239 & $2.85 \mathrm{E}-02$ & $1.88 \mathrm{E}-01$ & & & $1.47 \mathrm{E}+01$ & $2.38 \mathrm{E}+00$ & $1.41 E+\infty$ & 5.26E-01 & 5.23E-01 & $5.40 \mathrm{E}-01$ & & $8.09 \mathrm{E}-01$ \\
\hline Pu240 & $4.29 \mathrm{E}-03$ & $4.35 E-02$ & & & $4.09 \mathrm{E}+00$ & $4.53 E-01$ & $2.89 E-01$ & $1.08 \mathrm{E}-01$ & $1.06 \mathrm{E}-01$ & 1.14E-01 & & $1.73 \mathrm{E}-01$ \\
\hline Pu241 & $2.60 \mathrm{E}-02$ & $4.43 \mathrm{E}-01$ & & & $1.06 \mathrm{E}+02$ & $5.21 \mathrm{E}+00$ & $3.02 E+00$ & $1.49 E+\infty 0$ & $1.38 \mathrm{E}+\infty$ & $1.60 E \div 00$ & & $2.26 \mathrm{E}+00$ \\
\hline $\mathrm{Am} 241$ & $3.94 E+00$ & $1.50 \mathrm{E}-01$ & $5.03 E+00$ & $1.45 \mathrm{E}+00$ & $1.02 \mathrm{E}+01$ & $1.38 \mathrm{E}+01$ & $9.44 E+\infty$ & $7.48 E+\infty 0$ & $3.86 \bar{E}+\infty$ & $5.62 E+00$ & $1.63 E+00$ & $7.89 E+00$ \\
\hline $\mathrm{Cm} 244$ & $8.53 \mathrm{E}-03$ & & $1.09 \mathrm{E}-02$ & 3.17E-03 & & $1.11 \mathrm{E}-02$ & $8.66 \mathrm{E}-03$ & $1.10 \mathrm{E}-02$ & 4.04E-03 & 6.35E-03 & 3.54E-03 & $9.89 \mathrm{E}-03$ \\
\hline Total & $1.85 \mathrm{E}+05$ & $3.39 \mathrm{E}+03$ & $2.37 \mathrm{E}+0 \mathrm{~S}$ & $6.94 \mathrm{E}+04$ & $2.10 E+04$ & $2.41 \mathrm{E}+05$ & $1.88 \mathrm{E}+05$ & $2.40 \mathrm{E}+0 \mathrm{~S}$ & $8.82 \mathrm{E}+04$ & $1.12 \mathrm{E}+05$ & $7.65 \mathrm{E}+04$ & $2.15 \mathrm{E}+0 \mathrm{~S}$ \\
\hline & & & & & & & & & & & & \\
\hline & & & & & & & & & & & & \\
\hline & & & & & & & & & & & & \\
\hline $\begin{array}{l}\text { Insoluble } \\
\text { (Ci) }\end{array}$ & $B X-111$ & BX-112 & BY-101 & BY- 102 & BY-103 & BY-104 & BY- 105 & BY-106 & BY- 107 & BY-108 & BY-109 & BY-110 \\
\hline C14 & $2.30 \mathrm{E}-01$ & $5.99 \mathrm{E}-03$ & $2.94 \mathrm{E}-01$ & $8.53 \mathrm{E}-02$ & $2.52 \mathrm{E}-02$ & $3.07 \mathrm{E}-01$ & 2.44E-01 & $2.98 \mathrm{E}-01$ & 1.09E-01 & $1.12 E-01$ & $9.53 E-02$ & $2.66 \mathrm{E}-01$ \\
\hline Sr90 & $1.51 E+05$ & $3.08 E+03$ & $2.36 \mathrm{E}+05$ & $9.66 \mathrm{E}+04$ & $4.84 E+05$ & 1.97E+05 & $1.65 \mathrm{E}+05$ & $2.51 \mathrm{E}+0 \mathrm{~S}$ & $1.08 E+05$ & $3.49 E+04$ & $6.94 E+04$ & $2.30 E+05$ \\
\hline Y90 & $1.51 E+05$ & $3.08 \mathrm{E}+03$ & $2.36 E+05$ & $9.66 \mathrm{E}+04$ & $4.84 E+05$ & $1.97 \mathrm{E}+05$ & 1.65E+05 & $2.51 \mathrm{E}+05$ & $1.08 \mathrm{E}+05$ & $3.49 E+04$ & $6.94 \mathrm{E}+04$ & $2.30 \mathrm{E}+05$ \\
\hline Tc99 & $1.04 E+02$ & $6.27 \mathrm{E}-01$ & $1.33 E+02$ & $3.86 \mathrm{E}+01$ & $6.27 \mathrm{E}+\infty$ & $1.36 \mathrm{E}+02$ & $1.06 \mathrm{E}+02$ & $1.35 \mathrm{E}+02$ & $4.92 \mathrm{E}+01$ & $5.95 E+01$ & $4.32 \mathrm{E}+01$ & $1.21 \mathrm{E}+02$ \\
\hline Cs 137 & $2.97 \mathrm{E}+04$ & $5.38 \mathrm{E}+02$ & $3.81 E+04$ & $1.10 \mathrm{E}+04$ & $1.83 E+03$ & $3.88 \mathrm{E}+04$ & $3.02 E+04$ & $3.85 E+04$ & $1.41 E+04$ & $1.82 E+04$ & $1.23 \mathrm{E}+04$ & $3.44 \mathrm{E}+04$ \\
\hline Ba137 & $2 . \bar{O} 2 \bar{E}+U_{4} q$ & $\overline{5} . \mathbf{i} \mathbf{E}+\bar{u} \bar{z}$ & $\overline{3} . \bar{\delta} \angle \bar{E}+\overline{\mathrm{U} A}$ & i.ū̄E+ט̄A & $i . \overline{7} 4 \bar{E}+\bar{U} \bar{S}$ & 3.0SE+0.4 & 2.00E+Û4 & $3.00 \mathrm{C} \div \mathrm{A} 4$ & i.3FETOAA & $1.7 \hat{E} \div \hat{0} 4$ & $1.17 c+04$ & $3.27 \mathrm{C} \div 04$ \\
\hline Np237 & $1.01 E+\infty$ & $1.10 \mathrm{E}-02$ & $1.29 E+\infty$ & $3.73 E-01$ & $6.55 \mathrm{E}-02$ & $1.31 \mathrm{E}+\infty$ & $1.02 \mathrm{E}+00$ & $1.31 \mathrm{E}+00$ & $4.77 \mathrm{E}-0 \mathrm{t}$ & $7.60 \mathrm{E}-01$ & 4.17E-05 & $4.17 \mathrm{E}+00$ \\
\hline Pu239 & $1.40 \mathrm{E}+\infty$ & $9.22 \mathrm{E}+00$ & $3.04 \mathrm{E}-02$ & $3.11 \mathrm{E}-03$ & $7.22 \mathrm{E}+02$ & $1.17 \mathrm{E}+02$ & $6.90 E+01$ & $2.58 \mathrm{E}+01$ & $2.56 E+01$ & $2.65 E+01$ & & $3.96 \mathrm{E}+01$ \\
\hline Pu240 & $1.96 \mathrm{E}-01$ & $1.99 \mathrm{E}+00$ & $6.93 \mathrm{E}-03$ & 1.35E-03 & $1.87 \mathrm{E}+02$ & $2.07 \mathrm{E}+01$ & $1.32 \mathrm{E}+01$ & $4.95 E+\infty$ & $4.86 E+\infty$ & $5.22 \mathrm{E}+00$ & & $7.92 \mathrm{E}+00$ \\
\hline Pu241 & $3.81 E-01$ & $6.50 \mathrm{E}+00$ & $9.94 E-03$ & $1.37 \mathrm{E}-03$ & $1.55 \mathrm{E}+03$ & $7.64 \mathrm{E}+01$ & 4.44E+01 & $2.18 \mathrm{E}+01$ & $2.03 E+01$ & $2.35 E+01$ & & $3.31 \mathrm{E}+01$ \\
\hline Am241 & $3.61 E+01$ & $1.38 \mathrm{E}+\infty$ & $4.61 \mathrm{E}+01$ & $1.33 E+01$ & $9.37 \mathrm{E}+01$ & $1.26 \mathrm{E}+02$ & $8.66 E+01$ & $6.86 \mathrm{E}+01$ & $3.54 E+01$ & $5.16 E+01$ & $1.50 \mathrm{E}+01$ & $7.24 E+01$ \\
\hline $\mathrm{Cm} 244$ & $8.45 \mathrm{E}-01$ & & $1.08 E+00$ & $3.14 E-01$ & $5.46 E-02$ & $1.10 \mathrm{E}+00$ & 8.57E-01 & $1.09 \mathrm{E}+00$ & $4.00 \mathrm{E}-01$ & $6.28 \mathrm{E}-01$ & $3.50 \mathrm{E}-01$ & 9.79E-01 \\
\hline Total & $3.59 \mathrm{E}+05$ & $7.22 \mathrm{E}+03$ & $5.46 E+05$ & $2.15 \mathrm{E}+05$ & $9.73 E+05$ & $4.71 \mathrm{E}+05$ & $3.90 \mathrm{E}+05$ & $5.78 E+05$ & $2.44 E+05$ & $1.06 \mathrm{E}+05$ & $1.63 \mathrm{E}+05$ & $5.28 \mathrm{E}+05$ \\
\hline
\end{tabular}


Table A-2. Radionuclide Inventory Estimates for Single-Shell Tanks

\begin{tabular}{|c|c|c|c|c|c|c|c|c|c|c|c|c|}
\hline $\begin{array}{c}\text { Soluble } \\
(\mathrm{Cl})\end{array}$ & $B Y-111$ & $B Y-112$ & C-101 & $C-102$ & $\mathrm{C}-103$ & C- 104 & C- 105 & C- 106 & C-107 & $C-108$ & C-109 & C-110 \\
\hline $\mathrm{C14}$ & $3.01 \mathrm{E}+01$ & $5.48 E+\infty 0$ & $3.90 \mathrm{E}+\infty 0$ & & $8.53 \mathrm{E}+01$ & & $9.15 \mathrm{E}+01$ & $1.52 E-01$ & $6.26 E-01$ & 5.07E-02 & $3,46 \mathrm{E}-01$ & \\
\hline Sr90 & $2.34 E+03$ & $5.29 \mathrm{E}+02$ & $4.90 \mathrm{E}+02$ & $7.32 \mathrm{E}+03$ & $2.84 E+03$ & $2.63 E+03$ & $2.00 E+04$ & $3.85 E+03$ & $5.96 E+02$ & $1.60 \mathrm{E}+02$ & $4.89 \mathrm{E}-01$ & $8.68 \mathrm{E}+01$ \\
\hline Y90 & $2.34 E+03$ & $5.29 \mathrm{E}+02$ & $4.90 \mathrm{E}+02$ & $7.32 \mathrm{E}+03$ & $2.84 E+03$ & $2.63 E+03$ & $2.00 \mathrm{E}+04$ & $3.85 \mathrm{E}+03$ & $5.96 \mathrm{E}+02$ & $1.60 \mathrm{E}+02$ & 4.89E-01 & $8.68 E+01$ \\
\hline Tc99 & $1.37 \mathrm{E}+02$ & $2.51 \mathrm{E}+01$ & $1.48 E+01$ & & & & $3.45 \mathrm{E}+02$ & & $6.69 E-01$ & & 4.61E-01 & \\
\hline Cs137 & $1.22 \mathrm{E}+05$ & $2.24 E+04$ & $4.18 E+04$ & & & & $6.40 \mathrm{E}+04$ & & $1.95 \mathrm{E}+03$ & $1.04 E+00$ & $1.25 E+03$ & \\
\hline Ba137 & $1.16 E+05$ & $2.13 E+04$ & $3.97 \mathrm{E}+04$ & & & & $6.08 E+04$ & & $1.86 \mathrm{E}+03$ & $9.92 \mathrm{E}-01$ & $1.19 E+03$ & \\
\hline Np237 & 1.47E-01 & $2.70 \mathrm{E}-02$ & $2.33 E-02$ & & & & $5.08 \mathrm{E}-03$ & & & & & \\
\hline Pu239 & $3.68 \mathrm{E}-03$ & & $1.94 E+00$ & $3.23 E+01$ & $4.76 \mathrm{E}+\infty$ & $1.11 E+01$ & $2.43 E+01$ & $4.90 E+\infty$ & $3.98 \mathrm{E}+00$ & $1.41 \mathrm{E}+\infty$ & $1.60 \mathrm{E}-02$ & $1.89 \mathrm{E}+00$ \\
\hline Pu240 & & & $5.42 E-01$ & $9.60 E+\infty$ & $1.41 E+\infty$ & $3.33 E+\infty$ & $6.56 \mathrm{E}+\infty$ & $1.31 E+\infty$ & $1.12 \mathrm{E}+00$ & $3.94 E-01$ & $1.45 E-03$ & $4.59 \mathrm{E}-01$ \\
\hline Pu241 & $1.01 \mathrm{E}-02$ & & $1.13 E+01$ & $2.85 E+02$ & $3.82 E+01$ & $1.32 E+02$ & $1.31 E+02$ & $2.73 E+01$ & $2.36 \mathrm{E}+01$ & $8.15 E+\infty$ & $4.84 E-03$ & $7.37 \mathrm{E}+\infty 0$ \\
\hline Am241 & $5.16 \mathrm{E}+00$ & 9.44E-01 & $2.98 \mathrm{E}+00$ & $6.55 \mathrm{E}+01$ & $8.31 E+00$ & $5.11 E+01$ & $6.24 E+02$ & $2.39 E+01$ & $7.12 E+00$ & $2.08 \mathrm{E}+00$ & $8.25 \mathrm{E}-03$ & $1.28 \mathrm{E}+\infty$ \\
\hline $\mathrm{Cm} 244$ & $1.12 \mathrm{E}-02$ & $2.06 E-03$ & & & & & 4.81E-01 & & & & & \\
\hline Total & $2.43 E+05$ & $4.47 \mathrm{E}+04$ & $8.24 E+04$ & $1.48 \mathrm{E}+04$ & $5.79 E+03$ & $5.36 \mathrm{E}+03$ & $1.66 \mathrm{E}+05$ & $7.73 \mathrm{E}+03$ & $5.02 \mathrm{E}+03$ & $3.29 \mathrm{E}+02$ & $2.45 \mathrm{E}+03$ & $1.80 E+02$ \\
\hline & & & & & & & & & & & & \\
\hline C14 & $3.04 E-01$ & $5.53 \mathrm{E}-02$ & $3.94 \mathrm{E}-02$ & & $8.62 \mathrm{E}-01$ & & $9.25 \mathrm{E}-01$ & $1.54 \mathrm{E}-03$ & $6.32 E-03$ & & $3.50 \mathrm{E}-03$ & \\
\hline Sr9o & $2.32 E+05$ & $5.24 E+04$ & $4.85 E+04$ & $7.24 \mathrm{E}+05$ & $2.81 \mathrm{E}+05$ & $2.60 \mathrm{E}+0 \mathrm{~S}$ & $1.98 E+06$ & $3.81 E+05$ & $5.90 \mathrm{E}+04$ & $1.58 \mathrm{E}+04$ & $4.84 E+01$ & $8.59 E+03$ \\
\hline Y9o & $2.32 \mathrm{E}+05$ & $5.24 E+04$ & $4.85 E+04$ & $7.24 \mathrm{E}+05$ & $2.81 E+05$ & $2.60 \mathrm{E}+05$ & $1.98 \mathrm{E}+06$ & $3.81 \mathrm{E}+05$ & $5.90 \mathrm{E}+04$ & $1.58 E+04$ & 4.84E+01 & $8.59 E+03$ \\
\hline To99 & $1.37 E+02$ & $2.51 E+01$ & $1.48 \mathrm{E}+01$ & & & & $3.45 \mathrm{E}+02$ & & $6.69 \mathrm{E}-01$ & & 4.61E-01 & \\
\hline Cs137 & $3.90 E+04$ & $7.15 E+03$ & 1.33E+04 & & & & $2.04 E+04$ & & $6.24 E+02$ & $3.33 E-01$ & $4.01 E+02$ & \\
\hline Вa13) & 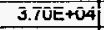 & 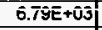 & $\mathbf{i} . \overline{\mathrm{I}} \overline{\mathrm{C}}+\overline{\mathrm{u}} \boldsymbol{A}$ & & & & 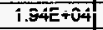 & & $5.935 \div \widetilde{0}$ & 3.17E-OI & 3.6iE+U2 & \\
\hline Np237 & $1.32 E+\infty$ & 2.43E-01 & $2.09 E-01$ & $4.40 \mathrm{E}-03$ & & $2.78 E-03$ & 4.58E-02 & $9.14 \mathrm{E}-03$ & 7.97E-03 & & $6.56 \mathrm{E}-03$ & \\
\hline Pu239 & $1.80 \mathrm{E}-01$ & & $9.49 E+01$ & $1.58 \mathrm{E}+03$ & $2.33 E+02$ & $5.44 E+02$ & $1.19 E+03$ & $2.40 \mathrm{E}+02$ & $1.95 \mathrm{E}+02$ & $6.93 E+01$ & $7.82 E-01$ & $9.27 \mathrm{E}+01$ \\
\hline Pu240 & $4.47 \mathrm{E}-02$ & $1.09 E-03$ & $2.48 E+01$ & $4.38 \mathrm{E}+02$ & $6.44 E+01$ & $1.52 \mathrm{E}+02$ & $3.00 \mathrm{E}+02$ & $5.99 E+01$ & $5.13 E+01$ & $1.80 E+01$ & $6.62 \mathrm{E}-02$ & $2.10 \mathrm{E}+01$ \\
\hline Pu241 & 1.48E-01 & & $1.65 \mathrm{E}+02$ & $4.19 \mathrm{E}+03$ & $5.60 \mathrm{E}+02$ & $1.94 \mathrm{E}+03$ & $1.92 E+03$ & $4.01 \mathrm{E}+02$ & $3.46 E+02$ & $1.19 E+02$ & $7.10 \mathrm{E}-02$ & $1.08 \mathrm{E}+02$ \\
\hline Am241 & $4.73 \mathrm{E}+01$ & $8.66 \mathrm{E}+00$ & $2.73 E+01$ & $6.01 E+02$ & $7.62 \mathrm{E}+01$ & $4.68 E+02$ & $5.73 \mathrm{E}+03$ & $2.20 \mathrm{E}+02$ & $6.53 E+01$ & $1.91 \mathrm{E}+01$ & $7.57 \mathrm{E}-02$ & $1.18 E+01$ \\
\hline $\mathrm{C}_{\mathrm{m} 244}$ & $1.11 \mathrm{E}+00$ & $2.04 \mathrm{E}-01$ & $1.11 \mathrm{E}-02$ & & & & $4.77 \mathrm{E}+01$ & & $4.72 \mathrm{E}-03$ & & & \\
\hline Total & $5.39 \mathrm{E}+05$ & 1.19E+0S & $1.23 E+05$ & $1.46 \mathrm{E}+06$ & $5.63 \mathrm{E}+05$ & $5.23 E+05$ & $4.01 E+06$ & $7.62 \mathrm{E}+05$ & $1.20 \mathrm{E}+05$ & $3.19 \mathrm{E}+04$ & $8.80 E+02$ & $1.74 E+04$ \\
\hline
\end{tabular}


Table A-2. Radionuclide Inventory Estimates for Single-Shell Tanks

\begin{tabular}{|c|c|c|c|c|c|c|c|c|c|c|c|c|}
\hline $\begin{array}{l}\text { Soluble } \\
\text { (Ci) }\end{array}$ & $\mathrm{C}-111$ & C-112 & $C-201$ & $\mathrm{C}-2 \mathrm{O} 2$ & C-203 & C.204 & s-101 & S-102 & S. 103 & S-104 & S. 105 & s-106 \\
\hline C14 & $1.28 \mathrm{E}-01$ & $3.69 \mathrm{E}-01$ & & & & & $8.34 E+00$ & & & 9.67E-01 & $2.62 E+00$ & $5.28 \mathrm{E}+00$ \\
\hline Sr9o & $6.36 \mathrm{E}+02$ & $2.67 \mathrm{E}+02$ & $4.36 \mathrm{E}-02$ & & $4.56 \mathrm{E}+00$ & $1.88 \mathrm{E}-03$ & $4.16 \mathrm{E}+03$ & $3.80 \mathrm{E}+02$ & & $3.45 E+03$ & $3.42 \mathrm{E}+02$ & $9.04 E+01$ \\
\hline \begin{tabular}{|l}
$Y 90$ \\
\end{tabular} & $6.36 \mathrm{E}+02$ & $2.67 E+02$ & $4.36 \mathrm{E}-02$ & & $4.56 \mathrm{E}+00$ & $1.88 \mathrm{E}-03$ & $4.16 E+03$ & $3.80 E+02$ & & $3.45 E+03$ & $3.42 E+02$ & $9.04 E+01$ \\
\hline Tc99 & 3.52E-01 & $2.91 E-01$ & & & & & $6.21 E+01$ & & & $3.20 E+\infty$ & $9.16 E+\infty$ & $1.78 E+01$ \\
\hline C8137 & $9.74 E+02$ & $8.42 E+02$ & 4.22E-01 & & & $1.82 \mathrm{E}-02$ & $2.18 E+04$ & & & $7.75 E+03$ & $2.13 \mathrm{E}+04$ & $4.20 E+04$ \\
\hline Ba137 & $9.26 \mathrm{E}+02$ & $8.00 \mathrm{E}+02$ & $4.01 E-01$ & & & $1.73 \mathrm{E}-02$ & $2.07 E+04$ & & & $7.36 E+03$ & $2.03 E+04$ & $3.99 E+04$ \\
\hline Np237 & & & & & & & $1.58 \mathrm{E}-02$ & & & $4.46 \mathrm{E}-03$ & $1.26 E-02$ & 2.44E-02 \\
\hline Pu239 & $1.04 E+00$ & $9.76 E-01$ & & & & & $3.04 E+00$ & $3.04 E-01$ & $3.40 \mathrm{E}-02$ & $2.88 E+\infty$ & $2.11 \mathrm{E}-01$ & $2.35 \mathrm{E}-02$ \\
\hline Pu240 & 2.63E-01 & $2.70 E-01$ & & & & & $7.31 \mathrm{E}-01$ & $7.31 \mathrm{E}-02$ & 8.13E-03 & $6.92 E-01$ & $5.27 \mathrm{E}-02$ & $5.66 \mathrm{E}-03$ \\
\hline Pu241 & $4.25 E+\infty$ & $5.21 E+00$ & & & & & $9.97 E+00$ & 9.97E-01 & $1.11 \mathrm{E}-01$ & $8.67 E+00$ & $6.25 \mathrm{E}-01$ & $6.97 \mathrm{E}-02$ \\
\hline Am241 & $1.26 \mathrm{E}+00$ & $1.40 \mathrm{E}+00$ & & 1.91E-03 & & & $2.49 E+01$ & $6.86 \mathrm{E}-01$ & $7.64 \mathrm{E}-02$ & $6.35 E+00$ & $5.78 E-01$ & $9.49 \mathrm{E}-02$ \\
\hline $\mathrm{Cm} 244$ & & & & & & & $8.10 E-03$ & & & & & \\
\hline Total & $3.18 \mathrm{E}+03$ & $2.18 \mathrm{E}+03$ & $9.12 E-01$ & $1.53 \mathrm{E}-02$ & $9.11 \mathrm{E}+00$ & 3.93E-02 & $5.09 E+04$ & $7.62 \mathrm{E}+02$ & $1.83 E-01$ & $2.20 \mathrm{E}+04$ & $4.23 \mathrm{E}+04$ & $8.21 \mathrm{E}+04$ \\
\hline & & & & & & & & & & & & \\
\hline & & & & & & & & & & & & \\
\hline & & & & & & & & & & & & \\
\hline $\begin{array}{c}\text { insoluble } \\
\text { (Ci) }\end{array}$ & $c-111$ & C-112 & C-201 & C-202 & C-203 & C-204 & S-101 & s-102 & s-103 & s-104 & s-105 & S-106 \\
\hline C14 & $1.30 \mathrm{E}-03$ & $3.72 \mathrm{E}-03$ & & & & & $8.43 E-02$ & & & 9.77E-03 & $2.64 E-02$ & $5.33 \mathrm{E}-02$ \\
\hline Sr90 & $6.30 \mathrm{E}+04$ & $2.64 E+04$ & $4.32 \mathrm{E}+\infty 0$ & & $4.51 E+02$ & $1.86 \mathrm{E}-01$ & $4.12 \mathrm{E}+05$ & $3.76 \mathrm{E}+04$ & & $3.42 E+05$ & $3.39 \mathrm{E}+04$ & $8.95 E+03$ \\
\hline \begin{tabular}{|l} 
Y9o \\
\end{tabular} & $6.30 \mathrm{E}+04$ & $2.64 \mathrm{E}+04$ & $4.32 E+00$ & & $4.51 E+02$ & $1.86 \mathrm{E}-01$ & $4.12 \mathrm{E}+05$ & $3.76 \mathrm{E}+04$ & & $3.42 E+05$ & $3.39 E+04$ & $8.95 E+03$ \\
\hline Tc99 & $3.52 E-01$ & $2.91 E-01$ & & & & & $6.21 E+01$ & & & $3.20 \mathrm{E}+00$ & $9.16 \mathrm{E}+\infty 0$ & $1.78 E+01$ \\
\hline Cs137 & $3.11 \mathrm{E}+02$ & $2.69 E+02$ & 1.35E-01 & & & $5.82 \mathrm{E}-03$ & $6.95 \mathrm{E}+03$ & & & $2.47 E+03$ & $6.82 E+\infty$ & $1.34 E+04$ \\
\hline 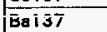 & $2.500+0 \overline{2}$ & $2.55 \mathrm{E}+\hat{0} 2$ & $i .20 \hat{E}-0 \hat{i}$ & & & 5.52E-63 & 6.6AE+03 & & & $2.355+03$ & 6.405:00 & $1.275+94$ \\
\hline Np237 & $5.26 \mathrm{E}-03$ & $4.40 \mathrm{E}-03$ & & & & & $1.42 \mathrm{E}-01$ & & & $4.01 \mathrm{E}-02$ & $1.13 \mathrm{E}-01$ & $2.20 \mathrm{E}-01$ \\
\hline Pu239 & $5.07 E+01$ & $4.78 E+01$ & $1.58 E-02$ & $4.34 E-02$ & $4.34 E-03$ & & $1.49 \mathrm{E}+02$ & $1.49 E+01$ & $1.66 \mathrm{E}+\infty 0$ & $1.41 E+02$ & $1.03 E+01$ & $1.15 \mathrm{E}+00$ \\
\hline Pu240 & $1.20 \mathrm{E}+01$ & $1.23 E+01$ & $1.38 \mathrm{E}-03$ & $3.79 \mathrm{E}-03$ & & & $3.34 \mathrm{E}+01$ & $3.34 \mathrm{E}+00$ & $3.71 E-01$ & $3.16 E+01$ & $2.41 E+\infty$ & $2.68 \mathrm{E}-01$ \\
\hline Pu241 & $6.23 \mathrm{E}+01$ & $7.65 E+01$ & $1.53 E-03$ & $4.21 E-03$ & & & $1.46 \mathrm{E}+02$ & $1.46 \mathrm{E}+01$ & $1.63 \mathrm{E}+00$ & $1.27 E+02$ & $9.17 E+\infty$ & $1.02 E+00$ \\
\hline Am241 & $1.16 \mathrm{E}+01$ & $1.28 E+01$ & $6.29 \mathrm{E}-03$ & $1.75 \mathrm{E}-02$ & $1.75 \mathrm{E}-03$ & & $2.28 \mathrm{E}+02$ & $6.29 E+00$ & 7.00E-01 & $5.82 E+01$ & $5.30 \mathrm{E}+00$ & 8.71E-01 \\
\hline Cm244 & & & & & & & $8.02 E-01$ & & & & $1.51 \mathrm{E}-03$ & $6.94 \mathrm{E}-03$ \\
\hline Total & $1.27 E+05$ & $5.35 \mathrm{E}+04$ & $8.93 \mathrm{E}+00$ & $2.94 \mathrm{E}-01$ & $9.02 \mathrm{E}+02$ & $3.84 \mathrm{E}-01$ & $8.38 \mathrm{E}+05$ & $7.53 \mathrm{E}+04$ & $4.59 \mathrm{E}+00$ & $6.88 \mathrm{E}+05$ & $8.11 \mathrm{E}+04$ & $4.41 \mathrm{E}+04$ \\
\hline
\end{tabular}


Table A-2. Radionuclide Inventory Estimates for Single-Shell Tanks

\begin{tabular}{|c|c|c|c|c|c|c|c|c|c|c|c|c|}
\hline $\begin{array}{c}\text { Soluble } \\
\text { (Ci) }\end{array}$ & S-107 & S-108 & $s-109$ & S-110 & S-111 & S-112 & sx-101 & sx-102 & sx-103 & sX-104 & sx-105 & $5 \times-106$ \\
\hline C14 & & $1.14 \mathrm{E}+01$ & $5.35 E+01$ & $3.73 E+01$ & $3.48 E+01$ & $2.28 \mathrm{E}+02$ & & $4.52 \mathrm{E}-02$ & $1.10 E+02$ & $1.23 \mathrm{E}+01$ & $2.68 E+01$ & $4.04 E+00$ \\
\hline Sr90 & $4.70 E+03$ & $5.28 E+02$ & $6.04 \mathrm{E}+02$ & $4.12 \mathrm{E}+03$ & $5.18 E+03$ & $1.02 \mathrm{E}+04$ & $5.18 E+03$ & $1.46 E+04$ & $5.04 E+03$ & $5.19 E+02$ & $2.65 E+03$ & $1.16 \mathrm{E}+03$ \\
\hline Y90 & $4.70 \mathrm{E}+03$ & $5.28 \mathrm{E}+02$ & $6.04 E+02$ & $4.12 E+03$ & $5.18 \mathrm{E}+03$ & $1.02 \mathrm{E}+04$ & $5.18 \mathrm{E}+03$ & $1.46 E+04$ & $5.04 E+03$ & $5.19 \mathrm{E}+02$ & $2.65 E+03$ & $1.16 \mathrm{E}+03$ \\
\hline Tc99 & & $5.19 E+01$ & $1.80 \mathrm{E}+02$ & $1.19 E+02$ & $1.11 \mathrm{E}+02$ & $7.77 \mathrm{E}+02$ & & $3.57 \mathrm{E}-01$ & $6.75 E+02$ & $9.43 E+01$ & 2.12E+02 & $7.98 E+01$ \\
\hline Cs137 & & $5.68 E+04$ & $3.20 \mathrm{E}+05$ & $3.57 \mathrm{E}+05$ & $3.29 \mathrm{E}+05$ & $1.11 \mathrm{E}+06$ & & $9.86 \mathrm{E}+01$ & $4.15 E+05$ & $2.83 E+04$ & $5.85 E+04$ & $5.71 \mathrm{E}+04$ \\
\hline Ba137 & & $5.40 E+04$ & $3.04 E+05$ & $3.39 \mathrm{E}+\mathrm{CS}$ & $3.13 E+05$ & $1.06 \mathrm{E}+06$ & & 9.37E+01 & $3.94 E+05$ & $2.69 E+04$ & $5.55 E+04$ & $5.42 E+04$ \\
\hline Np237 & & $2.66 \mathrm{E}-02$ & 1.46E-01 & $3.38 \mathrm{E}-02$ & 3.32E-02 & 4.44E-01 & & & 1.96E-01 & 2.37E-02 & $5.14 E-02$ & $5.49 E-02$ \\
\hline Pu239 & $1.77 \mathrm{E}+01$ & 4.36E-01 & $4.83 \mathrm{E}-02$ & $1.47 \mathrm{E}+\infty$ & $1.47 \mathrm{E}-01$ & $1.63 \mathrm{E}-02$ & $3.11 \mathrm{E}+\infty 0$ & $3.09 E-01$ & $3.44 E-02$ & $3.07 E+00$ & 2.59E-01 & \\
\hline Pu 240 & $4.43 E+\infty 0$ & $1.06 \mathrm{E}-01$ & $1.20 E-02$ & $3.68 \mathrm{E}-01$ & $3.68 \mathrm{E}-02$ & $4.21 \mathrm{E}-03$ & $6.85 E-01$ & $6.81 E-02$ & $7.80 \mathrm{E}-03$ & 6.32E-01 & 5.57E-02 & \\
\hline Pu241 & $9.71 E+01$ & $2.22 \mathrm{E}+00$ & $2.47 E-01$ & $4.41 E+\infty$ & 4.41E-01 & $4.91 E-02$ & $9.71 E+00$ & $9.71 E-01$ & $1.08 \mathrm{E}-01$ & $9.00 E+00$ & $8.02 E-01$ & \\
\hline $\operatorname{Am} 241$ & $2.39 \mathrm{E}+01$ & $9.80 \mathrm{E}+\infty 0$ & $1.27 \mathrm{E}+01$ & $3.82 \mathrm{E}+00$ & $8.98 \mathrm{E}-01$ & $6.60 \mathrm{E}+01$ & $9.13 \mathrm{E}+00$ & $1.02 E+\infty$ & $1.77 \mathrm{E}+02$ & $3.60 E+01$ & $6.29 E+01$ & $2.70 E+01$ \\
\hline $\mathrm{Cm} 244$ & & 4.08E-03 & $5.33 E-03$ & $1,58 \mathrm{E}-03$ & $1.56 \mathrm{E}-03$ & $3.64 E-02$ & & & $8.02 \mathrm{E}-02$ & $1.24 E-02$ & $2.83 E-02$ & $5.49 E-03$ \\
\hline Total & $9.49 \mathrm{E}+03$ & $1.12 E+05$ & $6.26 \mathrm{E}+05$ & $7.05 E+05$ & $6.53 E+05$ & $2.19 E+06$ & $1.04 \mathrm{E}+04$ & $2.95 E+04$ & $8.21 E+0 S$ & $5.64 E+04$ & 1.20E+05 & $1.14 \mathrm{E}+05$ \\
\hline & & & & & & & & & & & & \\
\hline & & & & & & & & & & & & \\
\hline & & & & & & & & & & & & \\
\hline $\begin{array}{c}\text { Insoluble } \\
\text { (Ci) }\end{array}$ & S-107 & $5-108$ & s-109 & s-110 & $S-111$ & s-112 & sX-101 & $S X-102$ & sX-103 & $5 x-104$ & $5 \times-105$ & sX-106 \\
\hline C14 & & 1.15E-01 & 5.41E-01 & $3.77 E-01$ & $3.52 E-01$ & $2.30 E+00$ & & & $1.11 E+00$ & $1.24 \mathrm{E}-01$ & 2.71E-01 & $4.08 E-02$ \\
\hline Sr90 & $4.65 E+05$ & $5.23 E+04$ & $5.98 E+04$ & $4.08 \mathrm{E}+05$ & $5.13 E+05$ & $1.01 E+06$ & $5.13 \mathrm{E}+05$ & $1.45 E+06$ & 4.99E+05 & $5.13 E+04$ & $2.62 E+05$ & $1.15 \mathrm{E}+05$ \\
\hline Y9O & $4.65 \mathrm{E}+05$ & $5.23 \mathrm{E}+04$ & $5.98 \mathrm{E}+04$ & $4.08 \mathrm{E}+05$ & $5.13 E+05$ & $1.01 \mathrm{E}+06$ & $5.13 E+05$ & $1.45 E+06$ & 4.99E+05 & $5.13 E+04$ & $2.62 \mathrm{E}+05$ & $1.15 \mathrm{E}+05$ \\
\hline Tc99 & & $5.19 \mathrm{E}+01$ & $1.80 \mathrm{E}+02$ & $1.19 \mathrm{E}+02$ & $1.11 E+02$ & $7.77 \mathrm{E}+02$ & & 3.57E-01 & $6.75 \mathrm{E}+02$ & $9.43 E+01$ & $2.12 E+02$ & $7.98 \mathrm{E}+01$ \\
\hline C\$137 & & $1.82 \mathrm{E}+04$ & $1.02 \mathrm{E}+05$ & $1.14 \mathrm{E}+05$ & $1.05 E+05$ & $3.55 \mathrm{E}+05$ & & $3.15 E+01$ & $1.33 \mathrm{E}+05$ & $9.04 E+03$ & $1.87 E+04$ & $1.82 E+04$ \\
\hline 6yi37 & & 1.72E+OG4 & 3.71E:04 & $4 . \mathrm{AOF}: 05$ & 3.F3ㄴ:04 & $3.37 ㄷ ㅡ: 05$ & & $2.905 \div 04$ & 1.20E $\div$ NS & Q.59든 & $1.776 \div 04$ & $1.735 \div 04$ \\
\hline Np237 & $2.22 \mathrm{E}-03$ & $2.39 \mathrm{E}-01$ & $1.31 E+00$ & $3.04 E-01$ & 2.99E-01 & $3.99 \mathrm{E}+00$ & $1.24 \mathrm{E}-03$ & & $1.76 \mathrm{E}+00$ & 2.13E-01 & 4.63E-01 & 4.94E-01 \\
\hline Pu239 & $8.68 \mathrm{E}+02$ & $2.14 \mathrm{E}+01$ & $2.37 \mathrm{E}+\infty$ & $7.19 E+01$ & $7.19 \mathrm{E}+00$ & $7.98 \mathrm{E}-01$ & $1.53 \mathrm{E}+02$ & $1.51 E+01$ & $1.69 E+\infty$ & $1.50 \mathrm{E}+02$ & $1.27 \mathrm{E}+01$ & \\
\hline Pu240 & $2.02 \mathrm{E}+02$ & $4.95 E+\infty 0$ & $5.49 \mathrm{E}-01$ & $1.68 \mathrm{E}+01$ & $1.68 \mathrm{E}+00$ & $1.92 \mathrm{E}-01$ & $3.13 \mathrm{E}+01$ & $3.11 E+\infty$ & $3.56 \mathrm{E}-01$ & $2.89 \mathrm{E}+01$ & $2.54 E+00$ & \\
\hline Pu241 & $1.42 \mathrm{E}+03$ & $3.26 \mathrm{E}+01$ & $3.62 \mathrm{E}+00$ & $6.47 \mathrm{E}+01$ & $6.47 E+00$ & $7.20 \mathrm{E}-01$ & $1.42 E+02$ & $1.42 E+01$ & $1.58 E+00$ & $1.32 \mathrm{E}+02$ & $1.18 \mathrm{E}+01$ & \\
\hline Am241 & $2.20 E+02$ & $8.99 E+01$ & $1.17 \mathrm{E}+02$ & $3.51 \mathrm{E}+01$ & $8.23 E+00$ & $6.06 \mathrm{E}+02$ & $8.38 \mathrm{E}+01$ & $9.37 \mathrm{E}+00$ & $1.63 E+03$ & $3.30 E+02$ & $5.77 \mathrm{E}+02$ & $2.48 \mathrm{E}+02$ \\
\hline $\mathrm{Cm} 244$ & & $4.04 E-01$ & 5.27E-01 & $1.57 \mathrm{E}-01$ & $1.55 \mathrm{E}-01$ & $3.60 \mathrm{E}+00$ & & $4.72 \mathrm{E}-03$ & $7.94 E+\infty 0$ & $1.23 E+00$ & $2.80 \mathrm{E}+00$ & $5.43 E-01$ \\
\hline Total & $9.33 E+05$ & $1.40 E+05$ & $3.19 E+05$ & $1.04 E+06$ & $1.23 \mathrm{E}+06$ & $2.71 E+06$ & $1.03 E+06$ & $2.90 \mathrm{E}+06$ & $1.26 \mathrm{E}+06$ & $1.21 \mathrm{E}+05$ & $5.61 \mathrm{E}+05$ & $2.66 \mathrm{E}+05$ \\
\hline
\end{tabular}


Table A-2. Radionuclide Inventory Estimates for Single-Shell Tanks

\begin{tabular}{|c|c|c|c|c|c|c|c|c|c|c|c|c|}
\hline $\begin{array}{c}\text { Soluble } \\
\text { (Ci) }\end{array}$ & $5 x-107$ & sX-108 & sX-109 & $5 \times-110$ & $5 \times-111$ & sX-112 & sX-113 & $5 x-114$ & $5 \times-115$ & $T-101$ & $T-102$ & $T-103$ \\
\hline C14 & & & & & & $7.28 \mathrm{E}-02$ & $1.19 \mathrm{E}+01$ & & $2.32 E+01$ & $1.34 E+01$ & $2.27 \mathrm{E}-01$ & $1.27 \mathrm{E}+00$ \\
\hline Sr90 & $1.29 \mathrm{E}+04$ & $8.03 \mathrm{E}+03$ & $2.42 E+03$ & $5.28 \mathrm{E}+03$ & $7.39 \mathrm{E}+03$ & $8.54 E+03$ & $2.25 \mathrm{E}+03$ & $7.75 E+03$ & $5.55 \mathrm{E}+03$ & $1.80 \mathrm{E}+01$ & $3.06 \mathrm{E}-0 \mathrm{t}$ & $1.51 \mathrm{E}+02$ \\
\hline Y90 & $1.29 \mathrm{E}+04$ & $8.03 E+03$ & $2.42 \mathrm{E}+03$ & $5.28 E+03$ & $7.39 \mathrm{E}+03$ & $8.54 E+03$ & $2.25 \mathrm{E}+03$ & $7.75 \mathrm{E}+03$ & $5.55 \mathrm{E}+03$ & $1.80 \mathrm{E}+01$ & $3.06 \mathrm{E}-01$ & $1.51 \mathrm{E}+02$ \\
\hline Tc99 & & & & & & $2.31 \mathrm{E}-01$ & $3.82 \mathrm{E}+01$ & & $7.44 E+01$ & $2.39 \mathrm{E}+01$ & $4.05 E-01$ & $2.24 E+00$ \\
\hline Cs137 & $1.65 \mathrm{E}-03$ & $3.18 E-03$ & $3.39 E-03$ & & & $6.89 E+02$ & $1.03 E+05$ & & $2.09 E+05$ & $6.19 \mathrm{E}+03$ & $1.05 \mathrm{E}+02$ & $5.03 E+02$ \\
\hline Ba137 & $1.57 \mathrm{E}-03$ & $3.02 \mathrm{E}-03$ & $3.22 \mathrm{E}-03$ & & & $6.54 E+02$ & $9.78 \mathrm{E}+04$ & & $1.98 E+05$ & $5.88 \mathrm{E}+03$ & $9.96 E+01$ & $4.78 E+02$ \\
\hline Np237 & & & & & & & $5.46 \mathrm{E}-02$ & & $2.91 E-02$ & $8.86 E-03$ & & \\
\hline Pu239 & $4.29 \mathrm{E}+00$ & $4.64 E+00$ & $2.83 E+00$ & $4.39 \mathrm{E}+00$ & $5.61 E+\infty$ & $3.21 \mathrm{E}+\infty$ & $1.02 \mathrm{E}+\infty$ & $7.10 \mathrm{E}+\infty$ & $1.82 \mathrm{E}+\mathrm{CO}$ & & & $1.76 \mathrm{E}-03$ \\
\hline Pu240 & $1.07 \mathrm{E}+00$ & $1.13 \mathrm{E}+00$ & 6.45E-01 & $1.18 \mathrm{E}+00$ & $1.49 E+\infty 0$ & $7.99 \mathrm{E}-01$ & 2.25E-01 & $1.81 \mathrm{E}+00$ & $4.74 E-01$ & & & \\
\hline Pu241 & $2.11 E+01$ & $2.26 \mathrm{E}+01$ & 1.20E+01 & $3.12 E+01$ & $4.51 \mathrm{E}+01$ & $1.52 \mathrm{E}+01$ & $3.02 \mathrm{E}+00$ & $3.73 E+01$ & $9.00 \mathrm{E}+00$ & & & $3.20 \mathrm{E}-03$ \\
\hline Am241 & $2.96 \mathrm{E}+01$ & $3.19 \mathrm{E}+01$ & $1.31 \mathrm{E}+01$ & $4.57 E+01$ & $5.88 \mathrm{E}+01$ & $1.84 \mathrm{E}+01$ & $2.49 \mathrm{E}+\infty 0$ & $4.90 \mathrm{E}+01$ & $1.01 \mathrm{E}+01$ & $5.88 E+\infty$ & $1.00 \mathrm{E}-01$ & $5.62 \mathrm{E}-01$ \\
\hline $\mathrm{Cm} 244$ & & & & & & & & & & $3.19 E-03$ & & \\
\hline Total & $2.59 \mathrm{E}+04$ & $1.61 E+04$ & $4.86 \mathrm{E}+03$ & $1.06 E+04$ & $1.49 E+04$ & $1.84 \mathrm{E}+04$ & $2.05 E+05$ & $1.56 \mathrm{E}+04$ & $4.18 \mathrm{E}+05$ & $1.22 E+04$ & $2.06 \mathrm{E}+02$ & $1.29 \mathrm{E}+03$ \\
\hline & & & & & & & & & & & & \\
\hline & & & & & & & & & & & & \\
\hline & & & & & & & & & & & & \\
\hline $\begin{array}{c}\text { Insoluble } \\
\text { (Ci) }\end{array}$ & $5 x-107$ & $5 \times-108$ & $5 \times-109$ & $5 x-110$ & $5 \times-111$ & sX-112 & SX-113 & $s x-114$ & $s x-115$ & $T-101$ & $T-102$ & $T-103$ \\
\hline C14 & & & & & & & $1.21 \mathrm{E}-01$ & & $2.35 \mathrm{E}-01$ & $1.35 \mathrm{E}-01$ & $2.29 E-03$ & $1.29 \mathrm{E}-02$ \\
\hline 5790 & $1.28 \mathrm{E}+06$ & $7.95 E+05$ & $2.39 E+05$ & $5.23 \mathrm{E}+05$ & $7.32 \mathrm{E}+05$ & $8.45 \mathrm{E}+05$ & $2.23 E+05$ & $7.67 E+05$ & $5.50 \mathrm{E}+05$ & $1.78 \mathrm{E}+03$ & $3.03 E+01$ & $1.50 E+04$ \\
\hline Y9o & $1.28 E+06$ & $7.95 E+05$ & $2.39 \mathrm{E}+05$ & $5.23 \mathrm{E}+05$ & $7.32 E+05$ & $8.45 \mathrm{E}+05$ & $2.23 E+05$ & $7.67 \mathrm{E}+05$ & $5.50 \mathrm{E}+05$ & $1.78 \mathrm{E}+03$ & $3.03 E+01$ & $1.50 E+04$ \\
\hline Tc99 & & & & & & $2.31 \mathrm{E}-01$ & $3.82 E+01$ & & $7.44 E+01$ & $2.39 \mathrm{E}+01$ & 4.05E-01 & $2.24 E+\infty 0$ \\
\hline Cs137 & & $1.01 E-03$ & $1.08 \mathrm{E}-03$ & & & $2.20 \mathrm{E}+02$ & $3.29 \mathrm{E}+04$ & & $6.66 \mathrm{E}+04$ & $1,98 \mathrm{E}+03$ & 3.35E+01 & $1.61 \mathrm{E}+02$ \\
\hline Ba:37 & & & 1.02502 & & & $2.005 \div 02$ & $3.12 E \div 04$ & & $6.33 E+04$ & $1.885 \div 03$ & $3.195 \div 04$ & $1.535 \div 02$ \\
\hline Np237 & $2.59 E-03$ & $2.85 E-03$ & $1.03 E-03$ & $2.46 \mathrm{E}-03$ & $4.79 E-03$ & $2.09 \mathrm{E}-03$ & $4.91 \mathrm{E}-01$ & $3.13 E-03$ & 2.62E-01 & 7.97E-02 & $4.35 \mathrm{E}-03$ & $6.65 E-03$ \\
\hline Pu239 & $2.10 \mathrm{E}+02$ & $2.28 E+02$ & $1.39 E+02$ & $2.15 \mathrm{E}+02$ & $2.75 E+02$ & $1.57 \mathrm{E}+02$ & $4.99 \mathrm{E}+01$ & $3.48 \mathrm{E}+02$ & $8.91 E+01$ & & & $8.62 \mathrm{E}-02$ \\
\hline Pu240 & $4.91 E+01$ & $5.18 E+01$ & $2.94 E+01$ & $5.40 \mathrm{E}+01$ & $6.80 E+01$ & $3.65 \mathrm{E}+01$ & $1.03 E+01$ & $8.28 \mathrm{E}+01$ & $2.17 \mathrm{E}+01$ & & & $1.24 \mathrm{E}-02$ \\
\hline Pu241 & $3.10 E+02$ & $3.32 \mathrm{E}+02$ & $1.76 \mathrm{E}+02$ & $4.58 \mathrm{E}+02$ & $6.62 E+02$ & $2.23 \mathrm{E}+02$ & $4.43 E+01$ & $5.47 \mathrm{E}+02$ & $1.32 \mathrm{E}+02$ & & & $4.69 \mathrm{E}-02$ \\
\hline Am241 & $2.72 \mathrm{E}+02$ & $2.92 \mathrm{E}+02$ & $1.20 \mathrm{E}+02$ & 4.19E+02 & $5.39 E+02$ & $1.68 \mathrm{E}+02$ & $2.28 E+01$ & $4.49 E+02$ & $9.23 E+01$ & $5.39 \bar{E}+01$ & $9.18 E-01$ & $5.16 \mathrm{E}+00$ \\
\hline Cm244 & & & & & & & $9.23 \mathrm{E}-0.3$ & & $5.87 \mathrm{E}-02$ & $3.16 E-01$ & 5.35E-03 & $3.01 E-02$ \\
\hline Total & $2.56 E+06$ & $1.59 E+06$ & $4.79 \mathrm{E}+05$ & $1.05 E+06$ & $1.46 \mathrm{E}+06$ & $1.69 \mathrm{E}+06$ & $5.10 \mathrm{E}+05$ & $1.54 E+06$ & $1.23 \mathrm{E}+06$ & $7.50 \mathrm{E}+03$ & $1.27 E+02$ & $3.03 E+04$ \\
\hline
\end{tabular}


Table A-2. Radionuclide Inventory Estimates for Single-Shell Tanks

\begin{tabular}{|c|c|c|c|c|c|c|c|c|c|c|c|c|}
\hline $\begin{array}{l}\text { Soluble } \\
\text { (Ci) }\end{array}$ & $T-104$ & T-105 & $\mathrm{T}-106$ & $T-107$ & $T-108$ & $T-109$ & $T-110$ & $T-111$ & $T-112$ & $T-201$ & $\mathrm{~T}-202$ & $T-203$ \\
\hline C14 & $3.02 E-02$ & & $1,87 \bar{E}-02$ & & $2.49 E-02$ & $2.23 E-02$ & & & & & & \\
\hline Sr9o & $2.86 \mathrm{E}+02$ & $3.48 E+01$ & $1.78 \mathrm{E}+\infty 0$ & $2.52 \mathrm{E}+02$ & $2.53 E+01$ & $5.09 \mathrm{E}-01$ & $2.59 \mathrm{E}+01$ & $2.20 \mathrm{E}+01$ & $3.26 \mathrm{E}+01$ & & & \\
\hline Y90 & $2.86 \mathrm{E}+02$ & $3.48 \mathrm{E}+01$ & $1.78 \mathrm{E}+00$ & $2.52 \mathrm{E}+02$ & $2.53 \mathrm{E}+01$ & $5,09 E-01$ & $2.59 \mathrm{E}+01$ & $2.20 \mathrm{E}+01$ & $3.26 \mathrm{E}+01$ & & & \\
\hline Tc99 & $1.08 \mathrm{E}+00$ & & $5.62 E-02$ & & $9,96 E-02$ & $6.21 \mathrm{E}-01$ & & & & & & \\
\hline Cs137 & $2.62 \mathrm{E}+03$ & & $1.48 \mathrm{E}+02$ & & $3.06 \mathrm{E}+02$ & $1.29 \mathrm{E}+03$ & & & & & & \\
\hline Ba137 & $2.49 E+03$ & & $1.41 E+02$ & & $2.91 E+02$ & $1.22 \mathrm{E}+03$ & & & & & & \\
\hline Np237 & $1.56 E-03$ & & & & & & & & & & & \\
\hline Pu239 & $2.12 \mathrm{E}+00$ & $2.76 E+\infty$ & 3.07E-01 & 7.38E-01 & $7.38 \mathrm{E}-02$ & 8.18E-03 & $2.81 E+\infty 0$ & $1.88 E+00$ & $3.11 \mathrm{E}+\infty$ & & $4.46 \mathrm{E}-03$ & $4.46 E-02$ \\
\hline Pu240 & $4.22 \mathrm{E}-01$ & $4.99 E-01$ & $5.54 E-02$ & 6.35E-02 & $6.35 \mathrm{E}-03$ & & 3.27E-01 & 4.04E-01 & $7.28 \mathrm{E}-01$ & & $1.14 E-03$ & 1.14E-02 \\
\hline Pu241 & $4.71 \mathrm{E}+00$ & $6.28 \mathrm{E}+00$ & 6.97E-01 & $2.01 \mathrm{E}-01$ & $2.01 E-02$ & 2.24E-03 & $1.66 \mathrm{E}+\infty 0$ & $4.06 \mathrm{E}+\infty$ & $9.45 E+\infty 0$ & & $1,29 \mathrm{E}-02$ & 1.29E-01 \\
\hline Am241 & $1.33 E+00$ & $1.38 E+00$ & $1.54 \mathrm{E}-01$ & 1.19E-01 & $1.20 \mathrm{E}-02$ & $1.34 E-03$ & $4.52 \mathrm{E}-01$ & $9.39 E-01$ & $1.92 \mathrm{E}+00$ & & $2.85 E-03$ & $2.85 E-02$ \\
\hline \multicolumn{13}{|l|}{$\mathrm{Cm} 244$} \\
\hline Total & $5.69 \mathrm{E}+03$ & $7.67 E+01$ & $2.93 \mathrm{E}+02$ & $5.05 E+02$ & $6.48 \mathrm{E}+02$ & $2.51 E+03$ & $5.59 \mathrm{E}+01$ & $4.84 E+01$ & $7.42 \mathrm{E}+01$ & & $1.24 \mathrm{E}-02$ & $1.24 \mathrm{E}-01$ \\
\hline & & & & & & & & & & & & \\
\hline & & & & & & & & & & & & \\
\hline & & & & & & & & & & & & \\
\hline $\begin{array}{c}\text { Insoluble } \\
\text { (Ci) }\end{array}$ & $\mathrm{T}-104$ & T-105 & $T-106$ & $T-107$ & $T-108$ & $T-109$ & $T-110$ & $T-111$ & $T \cdot 112$ & $T-201$ & $\mathrm{~T}-202$ & $T-203$ \\
\hline \multicolumn{13}{|l|}{ C14 } \\
\hline Sr9o & $2.83 E+04$ & $3.44 E+03$ & $1.76 E+02$ & $2.49 \mathrm{E}+04$ & $2.50 \mathrm{E}+03$ & $5.04 \mathrm{E}+01$ & $2.56 \mathrm{E}+03$ & $2.17 E+03$ & $3.23 E+03$ & & & \\
\hline Y90 & $2.83 E+04$ & $3.44 E+03$ & $1.76 E+02$ & $2.49 \mathrm{E}+04$ & $2.50 \mathrm{E}+03$ & $5.04 E+01$ & $2.56 \mathrm{E}+03$ & $2.17 \bar{E}+03$ & $3.23 \mathrm{E}+03$ & & & \\
\hline Tc99 & $1.08 \mathrm{E}+00$ & & $5.62 \mathrm{E}-02$ & & $9.96 \mathrm{E}-02$ & $6.21 \mathrm{E}-01$ & & & & & & \\
\hline Cs137 & $8.37 E+02$ & & $473 \mathrm{E}+01$ & & $97 \mathrm{AE}+01$ & $4.10 E+02$ & & & & & & \\
\hline Ba137 & $7.95 E+02$ & & $4.49 E+01$ & & $9.29 \mathrm{E}+01$ & $3.90 \mathrm{E}+02$ & & & & & & \\
\hline Np237 & $1.40 \mathrm{E}-02$ & & & & $2.20 \mathrm{E}-03$ & $7.30 \mathrm{E}-03$ & & & & & & \\
\hline Pu239 & $1.04 E+02$ & $1.35 \mathrm{E}+02$ & $1.50 E+01$ & $3.62 \mathrm{E}+01$ & $3.62 \mathrm{E}+00$ & $4.01 \mathrm{E}-01$ & $1.37 \mathrm{E}+02$ & $9.22 \mathrm{E}+01$ & $1.53 \mathrm{E}+02$ & & $2.18 \mathrm{E}-01$ & $2.18 \mathrm{E}+00$ \\
\hline Pu240 & $1.93 E+01$ & $2.28 \mathrm{E}+01$ & $2.53 \mathrm{E}+00$ & $2.90 E+\infty 0$ & $2.90 \mathrm{E}-01$ & $3.22 \mathrm{E}-02$ & $7.49 E+01$ & $1.85 E+01$ & $3.33 \mathrm{E}+01$ & & $5.22 \mathrm{E}-02$ & $5.22 \mathrm{E}-01$ \\
\hline Pu241 & $6.91 \mathrm{E}+01$ & $9.22 \mathrm{E}+01$ & $1.02 \mathrm{E}+01$ & $2.94 \mathrm{E}+00$ & 2.94E-01 & $3.28 \mathrm{E}-02$ & $2.43 E+01$ & $5.96 \mathrm{E}+01$ & $1.39 \mathrm{E}+02$ & & $1.89 \mathrm{E}-01$ & $1.89 \mathrm{E}+00$ \\
\hline Am 241 & $1.22 \mathrm{E}+01$ & 1.27E+01 & $1.41 \mathrm{E}+00$ & $1.09 \mathrm{E}+00$ & $1.10 \mathrm{E}-01$ & $1.23 \mathrm{E}-02$ & $4.14 \mathrm{E}+\infty$ & $8.61 E+00$ & $1.76 E+01$ & & 2.62E-02 & 2.62E-01 \\
\hline \multicolumn{13}{|l|}{$\mathrm{Cm} 244$} \\
\hline Total & $5.84 E+04$ & $7.18 \mathrm{E}+03$ & $4.77 \mathrm{E}+02$ & $4.99 \mathrm{E}+04$ & $5.20 E+03$ & $9.02 \mathrm{E}+02$ & $5.31 \mathrm{E}+03$ & $4.53 E+03$ & $6.82 E+03$ & & 4.88E-01 & $4.88 \mathrm{E}+00$ \\
\hline
\end{tabular}


Table A-2. Radionuclide Inventory Estimates for Single-Shell Tanks

\begin{tabular}{|c|c|c|c|c|c|c|c|c|c|c|c|c|}
\hline $\begin{array}{c}\text { Soluble } \\
\text { (Ci) }\end{array}$ & $T-204$ & TX-101 & $T X-102$ & $T X-103$ & TX-104 & $T X-105$ & TX-106 & TX-107 & TX-108 & TX-109 & $T X-110$ & TX-111 \\
\hline$C 14$ & & $3.00 E+00$ & $4.98 \mathrm{E}-01$ & 1.44E-01 & & $4.67 \mathrm{E}+00$ & $9.41 \mathrm{E}-01$ & & $2.64 E+00$ & $5.65 E+01$ & $2.03 E+01$ & $2.18 E+\infty 0$ \\
\hline Sr9o & & $6.11 \mathrm{E}+01$ & $2.34 \mathrm{E}+00$ & $5.20 \mathrm{E}+00$ & & $2.29 \mathrm{E}+0 \mathrm{t}$ & & & $3.68 \mathrm{E}+01$ & $1.05 E+03$ & $7.39 \mathrm{E}+02$ & $3.49 E+02$ \\
\hline Y90 & & $6.11 \mathrm{E}+01$ & $2.34 \mathrm{E}+\infty$ & $5.20 \mathrm{E}+00$ & & $2.29 \mathrm{E}+01$ & & & $3.68 \mathrm{E}+01$ & $1.05 E+03$ & $7.39 \mathrm{E}+02$ & $3.49 E+02$ \\
\hline To99 & & & $3.32 E+\infty 0$ & $4.77 \mathrm{E}-01$ & & 3.05E+01 & $3.01 E+\infty$ & & 1.14E+01 & $2.90 \mathrm{E}+02$ & $1.04 E+02$ & $1.54 E+01$ \\
\hline Cs 137 & & & $5.15 \mathrm{E}+02$ & $7.14 E+02$ & & $4.99 \mathrm{E}+03$ & $8.07 E+03$ & & $2.99 \mathrm{E}+04$ & $2.15 E+05$ & $9.02 E+04$ & $2.04 E+04$ \\
\hline Be137 & & & $4.89 \mathrm{E}+02$ & $6.79 \mathrm{E}+02$ & & $4.74 \mathrm{E}+03$ & $7.67 \mathrm{E}+03$ & & $2.84 E+04$ & $2.04 E+05$ & $8.57 E+04$ & $1.94 E+04$ \\
\hline Np237 & & & $2.76 E-03$ & & & $2.50 \mathrm{E}-02$ & $4.46 \mathrm{E}-03$ & & 9.44E-03 & $2.66 \mathrm{E}-01$ & $9.78 \mathrm{E}-02$ & $1.62 \mathrm{E}-02$ \\
\hline Pu239 & & & & & & & & & 9.62E-03 & $2.38 \mathrm{E}+00$ & $1.78 \mathrm{E}-01$ & $1.87 \mathrm{E}-02$ \\
\hline Pu240 & & & & & & & & & $2.68 \mathrm{E}-03$ & 4.90E-01 & $3.48 \mathrm{E}-02$ & $3.73 \mathrm{E}-03$ \\
\hline Pu241 & & & & & & & & & $4.68 \mathrm{E}-02$ & $5.44 E+00$ & $3.47 E-01$ & $3.76 \mathrm{E}-02$ \\
\hline Am241 & & & & $6.81 \mathrm{E}-03$ & & & & & $6.09 \mathrm{E}-02$ & $8.67 E+\infty$ & $2.95 E+00$ & $3.02 E-01$ \\
\hline $\mathrm{Cm} 244$ & & & & & & & & & & 8.34E-03 & $3.19 \mathrm{E}-03$ & \\
\hline Total & & $1.25 E+02$ & $1.01 \mathrm{E}+03$ & $1.40 \mathrm{E}+03$ & & $9.81 \mathrm{E}+03$ & $1.57 \mathrm{E}+04$ & & $5.84 E+04$ & $4.21 \mathrm{E}+05$ & $1.78 E+05$ & $4.06 \mathrm{E}+04$ \\
\hline & & & & & & & & & & & & \\
\hline & & & & & & & & & & & & \\
\hline $\begin{array}{c}\text { Insoluble } \\
\text { (Ci) }\end{array}$ & $T-204$ & TX-101 & $T X-102$ & $T X-103$ & TX-104 & $T X-105$ & $T X-106$ & TX-107 & $\mathrm{TX}-108$ & $T X-109$ & $\mathrm{TX}-110$ & $\mathrm{TX}-111$ \\
\hline$C 14$ & & $3.03 E-02$ & $5.03 E-03$ & $1.46 E-03$ & & $4.71 E-02$ & $9.51 E-03$ & & $2.66 \mathrm{E}-02$ & $5.70 \mathrm{E}-01$ & 2.05E-01 & $2.20 \mathrm{E}-02$ \\
\hline Sr9o & & $6.04 E+03$ & $2.32 \mathrm{E}+02$ & $5.15 \mathrm{E}+02$ & & $2.27 \mathrm{E}+03$ & & & $3.64 E+03$ & $1.04 E+05$ & $7.32 E+04$ & $3.45 E+04$ \\
\hline Y90 & & $6.04 E+03$ & $2.32 E+02$ & $5.15 \mathrm{E}+02$ & & $2.27 E+03$ & & & $3.64 E+03$ & $1.04 E+05$ & $7.32 \mathrm{E}+04$ & $3.45 E+04$ \\
\hline Tc99 & & & $3.32 \mathrm{E}+00$ & 4.77E-01 & & $3.05 E+01$ & $3.01 E+\infty 0$ & & 1.14E+01 & $2.90 \mathrm{E}+02$ & $1.04 E+02$ & $1.54 E+01$ \\
\hline $\operatorname{cs137}$ & & & $1.64 E+02$ & $2.28 \mathrm{E}+02$ & & $1.595 \div 03$ & $2.585 \div 03$ & & $3.5 \leqslant 5+\infty$ & $6.265 \div 04$ & $2.88 E+04$ & $6.53 E \div 03$ \\
\hline Ba137 & & & $1.56 \mathrm{E}+02$ & $2.17 E+02$ & & $1.51 E+03$ & $2.45 E+03$ & & $9.08 E+03$ & $6.52 \mathrm{E}+04$ & $2.74 \mathrm{E}+04$ & $6.20 \mathrm{E}+03$ \\
\hline Np237 & & & $2.48 \mathrm{E}-02$ & $4.66 \mathrm{E}-03$ & & $2.34 E-01$ & $4.01 \mathrm{E}-02$ & & $8.50 \mathrm{E}-02$ & $2.39 \mathrm{E}+\infty 0$ & $8.80 \mathrm{E}-01$ & 1.46E-01 \\
\hline Pu239 & & & & & & & & & $4.71 \mathrm{E}-01$ & $1.17 \mathrm{E}+02$ & $8.70 \mathrm{E}+\infty \mathrm{O}$ & 9.17E-01 \\
\hline Pu240 & & & & & & & & & $1.22 \mathrm{E}-01$ & $2.24 E+01$ & $1.59 \mathrm{E}+\infty 0$ & $1.70 E-01$ \\
\hline Pu241 & & & & & & 1.85E-03 & & & $6.86 \mathrm{E}-01$ & $7.97 \mathrm{E}+01$ & $5.09 \mathrm{E}+00$ & $5.52 E-01$ \\
\hline Am 241 & & & & $6.25 \mathrm{E}-02$ & & $1.96 \mathrm{E}-03$ & & & $5.58 \mathrm{E}-01$ & $7.95 \mathrm{E}+01$ & $2.70 E+01$ & $2.77 \mathrm{E}+00$ \\
\hline Cm244 & & & $3.70 \mathrm{E}-03$ & & & $3.49 \mathrm{E}-02$ & $1.13 \mathrm{E}-03$ & & $3.40 \mathrm{E}-02$ & $8.26 \mathrm{E}-01$ & 3.16E-01 & $3.20 \mathrm{E}-02$ \\
\hline Total & & $1.21 \mathrm{E}+04$ & $7.87 \mathrm{E}+02$ & $1.48 \mathrm{E}+03$ & 3.15E-03 & $7.67 \mathrm{E}+03$ & $5.03 E+03$ & $1.56 \mathrm{E}-03$ & $2.59 E+04$ & $3.43 E+05$ & $2.03 E+05$ & $8.18 \mathrm{E}+04$ \\
\hline
\end{tabular}


Table A-2. Radionuclide Inventory Estimates for Single-Shell Tanks

\begin{tabular}{|c|c|c|c|c|c|c|c|c|c|c|c|c|}
\hline $\begin{array}{c}\text { Soluble } \\
\text { (Ci) }\end{array}$ & $T X-112$ & $T X-113$ & $T X-114$ & $T X-115$ & TX-116 & $T X-117$ & $T X-118$ & Tr-101 & Tr-102 & TY-103 & TY-104 & TY.105 \\
\hline $\mathrm{C} 14$ & $3.52 \mathrm{E}-01$ & $2.36 \mathrm{E}+00$ & $6.59 E-01$ & $1.27 E+00$ & $1.72 E-01$ & $2.01 E+00$ & $1.93 E+02$ & $5.18 \mathrm{E}-01$ & $1.22 \mathrm{E}+01$ & $3.25 E+01$ & 4.54E-02 & $2.75 E+00$ \\
\hline Sr9o & $5.52 \mathrm{E}+01$ & $5.92 E+01$ & $5.55 E+\infty$ & $2.16 \mathrm{E}+02$ & $2.81 \mathrm{E}+02$ & $5.34 E+02$ & $5.50 \mathrm{E}+03$ & $1.23 \mathrm{E}+02$ & $8.68 \mathrm{E}+01$ & $7.82 E+02$ & $4.43 E+01$ & $1.84 E+03$ \\
\hline Y90 & $5.52 \mathrm{E}+01$ & $5.92 E+01$ & $5.55 \mathrm{E}+\infty$ & $2.16 \mathrm{E}+02$ & $2.81 E+02$ & $5.34 E+02$ & $5.50 \mathrm{E}+03$ & $1.23 E+02$ & $8.68 E+01$ & $7.82 E+02$ & $4.43 E+01$ & $1.84 E+03$ \\
\hline To99 & $7.87 \mathrm{E}+\infty$ & $2.62 \mathrm{E}+\infty$ & $7.34 E+\infty$ & $3.50 E+\infty$ & $1.77 E+\infty$ & $7.18 \mathrm{E}-01$ & $2.73 E+02$ & $6.59 \mathrm{E}-01$ & $7.77 E+01$ & $2.16 \mathrm{E}+02$ & $1.42 \mathrm{E}-01$ & $3.03 E+00$ \\
\hline Cs137 & $1.58 \mathrm{E}+04$ & $5.43 \mathrm{E}+03$ & $1.58 E+04$ & $8.84 E+03$ & $3.76 \mathrm{E}+03$ & $1.52 \mathrm{E}+03$ & $2.51 \mathrm{E}+05$ & $1.46 \mathrm{E}+03$ & $1.68 E+04$ & $3.36 \mathrm{E}+04$ & $3.83 E+02$ & $6.68 \mathrm{E}+03$ \\
\hline Be137 & $1.51 \mathrm{E}+04$ & $5.16 E+03$ & $1.50 \mathrm{E}+04$ & $8.40 E+03$ & $3.57 E+03$ & $1.45 \mathrm{E}+03$ & $2.38 \mathrm{E}+05$ & $1,39 E+03$ & $1.60 \mathrm{E}+04$ & $3.20 E+04$ & $3.64 E+02$ & $6.35 E+03$ \\
\hline Np237 & $9.82 E-03$ & $3.30 \mathrm{E}-03$ & $9.15 E-0.3$ & $3.15 \mathrm{E}-03$ & 2.21E-03 & & $2.52 \mathrm{E}-01$ & & 6.55E-02 & 1.79E-01 & & $3.81 \mathrm{E}-03$ \\
\hline Pu239 & $1.02 \mathrm{E}-03$ & $7.45 E-01$ & $7.45 E-02$ & $8.28 E-03$ & & & $1.45 E+01$ & $2.88 \mathrm{E}+\infty 0$ & $5.42 \mathrm{E}-02$ & $4.10 E+00$ & $1.28 \mathrm{E}-01$ & $1.09 \mathrm{E}+00$ \\
\hline Pu240 & & $1.38 \mathrm{E}-01$ & $1.38 \mathrm{E}-02$ & $1.54 E-03$ & & & $4.01 E+00$ & 7.32E-01 & $1.05 E-02$ & $6.64 E-01$ & $2.85 E-02$ & $1.46 \mathrm{E}-01$ \\
\hline Pu241 & & $1.14 \mathrm{E}+\infty 0$ & $1.14 \mathrm{E}-01$ & $1.27 \mathrm{E}-02$ & & & $1.56 E+02$ & $1.14 E+01$ & 1.54E-01 & $8.54 E+00$ & $4.40 \mathrm{E}-01$ & $8.80 \mathrm{E}-01$ \\
\hline Am241 & $1.77 \mathrm{E}-02$ & $3.79 E-01$ & $3.79 \mathrm{E}-02$ & 4.27E-03 & & $1.01 \mathrm{E}-03$ & $1.04 E+02$ & $2.80 E+\infty 0$ & $1.40 \mathrm{E}-02$ & $2.26 \mathrm{E}+00$ & 1.40E-01 & $1.52 E+00$ \\
\hline Cm244 & & & & & & & 9.51E-03 & & & $2.43 E-03$ & & \\
\hline Total & $3.10 \mathrm{E}+04$ & $1.07 \mathrm{E}+04$ & $3.08 \mathrm{E}+04$ & $1.77 \mathrm{E}+04$ & $7.89 \mathrm{E}+03$ & $4.04 \mathrm{E}+03$ & $5.00 \mathrm{E}+05$ & $3.11 \mathrm{E}+03$ & $3.31 \tilde{E}+04$ & $6.74 E+04$ & $8.36 \mathrm{E}+02$ & $1.67 E+04$ \\
\hline & & & & & & & & & & & & \\
\hline & & & & & & & & & & & & \\
\hline & & & & & & & & & & & & \\
\hline $\begin{array}{c}\text { Insoluble } \\
\text { (Ci) }\end{array}$ & $\mathrm{TX}-112$ & $T X-113$ & $T X-114$ & $T X-115$ & $T X-116$ & TX-117 & $T X-118$ & TY-101 & TY-102 & TY-103 & $T Y-104$ & $T Y-105$ \\
\hline $\mathrm{C} 14$ & $3.55 E-03$ & $2.38 \mathrm{E}-02$ & $6.66 \mathrm{E}-03$ & $1.29 \mathrm{E}-02$ & $1.74 \mathrm{E}-03$ & $2.03 E-02$ & $1.95 \mathrm{E}+00$ & $5.24 \mathrm{E}-03$ & $1.23 \mathrm{E}-01$ & $3.28 \mathrm{E}-01$ & & $2.78 \mathrm{E}-02$ \\
\hline Sr9o & $5.47 E+03$ & $5.86 \mathrm{E}+03$ & $5.49 \mathrm{E}+02$ & $2.14 \mathrm{E}+04$ & $2.78 E+04$ & $5.28 E+04$ & $5.45 \mathrm{E}+05$ & $1.21 \mathrm{E}+04$ & $8.59 \mathrm{E}+03$ & $7.74 E+04$ & $4.39 \mathrm{E}+03$ & $1.82 \mathrm{E}+05$ \\
\hline Y9o & $5.47 \mathrm{E}+03$ & $5.86 \mathrm{E}+03$ & $5.49 E+02$ & $2.14 E+04$ & $2.78 E+04$ & $5.28 \mathrm{E}+04$ & $5.45 E+05$ & $1.21 \mathrm{E}+04$ & $8.59 E+03$ & $7.74 E+04$ & $4.39 E+03$ & $1.82 E+05$ \\
\hline Tc99 & $7.87 \mathrm{E}+00$ & $2.62 E+\infty 0$ & $7.34 \mathrm{E}+00$ & $3.50 \mathrm{E}+00$ & 1.77E $+\infty$ & $7.18 \mathrm{E}-01$ & $2.73 E+02$ & $6.59 \mathrm{E}-01$ & $7.77 E+01$ & $2.16 E+02$ & $1.42 \mathrm{E}-01$ & $3.03 E+00$ \\
\hline Cs137 & $5.06 \mathrm{E} \div 03$ & $1.73 E \div 03$ & $5.05 E+03$ & $2.82 \mathrm{E} \div 03$ & $1.20 \mathrm{E}+03$ & $4.87 \mathrm{E} \div 02$ & $8.00 E+04$ & $467 E+02$ & $5.37 \mathrm{C}+03$ & $1.07 t+04$ & $1.22 \mathrm{E}+02$ & $2.13 E \div 03$ \\
\hline Be137 & $4.81 E+03$ & $1.65 \mathrm{E}+03$ & $4.79 \mathrm{E}+03$ & $2.68 \mathrm{E}+03$ & $1.14 \mathrm{E}+03$ & $4.62 E+02$ & $7.60 E+04$ & $4.43 E+02$ & $5.10 \mathrm{E}+03$ & $1.02 E+04$ & $1.16 \mathrm{E}+02$ & $2.03 E+03$ \\
\hline Np237 & $8.83 E-02$ & $2.97 \mathrm{E}-02$ & $8.24 E-02$ & $2.83 \mathrm{E}-02$ & $1.99 \mathrm{E}-02$ & $8.11 \mathrm{E}-03$ & $2.27 E+00$ & $7.81 \mathrm{E}-03$ & $5.90 \mathrm{E}-01$ & $1.61 \mathrm{E}+00$ & $1.62 \mathrm{E}-03$ & 3.43E- 02 \\
\hline Pu239 & $5.00 E-02$ & $3.65 \mathrm{E}+01$ & $3.65 \mathrm{E}+00$ & $4.06 \mathrm{E}-01$ & & & $7.09 E+02$ & $1.41 \mathrm{E}+02$ & $2.66 \mathrm{E}+00$ & $2.01 E+02$ & $6.27 E+00$ & $5.33 \mathrm{E} \div 01$ \\
\hline Pu240 & $6.57 \mathrm{E}-03$ & $6.30 \mathrm{E}+00$ & $6.30 \mathrm{E}-01$ & $7.02 \mathrm{E}-02$ & & & $1.83 E+02$ & $3.34 E+01$ & $4.82 E-01$ & $3.03 E+01$ & $1.30 \mathrm{E}+00$ & $6.66 E+\infty 0$ \\
\hline \begin{tabular}{|l|} 
Pu241 \\
\end{tabular} & $1.26 \mathrm{E}-02$ & $1.67 \mathrm{E}+01$ & $1.67 \mathrm{E}+00$ & $1.86 \mathrm{E}-01$ & & & $2.28 E+03$ & $1.67 \mathrm{E}+02$ & $2.26 E+\infty$ & $1.25 E+02$ & $6.45 \mathrm{E}+00$ & $1.29 \mathrm{E}+01$ \\
\hline Am241 & $1.62 E-01$ & $3.47 \mathrm{E}+\infty 0$ & $3.48 \mathrm{E}-01$ & $3.92 \mathrm{E}-\mathrm{C} 2$ & $5.68 \mathrm{E}-03$ & $9.23 \mathrm{E}-03$ & $9.51 E+02$ & $2.56 \mathrm{E}+01$ & $1.28 E-01$ & $2.08 E+01$ & $1.29 \mathrm{E}+00$ & $1.40 E+01$ \\
\hline $\mathrm{Cm} 244$ & $1.98 E-03$ & & & $2.23 \mathrm{E}-03$ & & & $9.41 \mathrm{E}-01$ & & $8.39 \mathrm{E}-02$ & $2.40 \mathrm{E}-01$ & & \\
\hline Total & $2.08 \mathrm{E}+04$ & $1.53 \mathrm{E}+04$ & $1.10 E+04$ & $4.83 \mathrm{E}+04$ & $5.79 \mathrm{E}+04$ & $1.07 \mathrm{E}+05$ & $1.25 E+06$ & $2.56 \mathrm{E}+04$ & $2.77 \mathrm{E}+04$ & $1.76 \mathrm{E}+05$ & $9.03 E+03$ & $3.68 \mathrm{E}+05$ \\
\hline
\end{tabular}


Table A-2. Radionuclide Inventory Estimates for Single-Shell Tanks

\begin{tabular}{|c|c|c|c|c|c|c|c|c|c|c|c|c|}
\hline $\begin{array}{l}\text { Soluble } \\
\text { (Ci) }\end{array}$ & TY-106 & $\mathrm{U}-101$ & $\mathrm{U}-102$ & U-103 & U-104 & U. 105 & U. 106 & U-107 & $U-108$ & U-109 & U-110 & U-111 \\
\hline $\mathrm{C}_{14}$ & $2.32 E-02$ & $1.88 E+\infty$ & $4.67 \mathrm{E}+\infty 0$ & & & $1.30 \mathrm{E}-0.3$ & & $1.40 \mathrm{E}+00$ & $1.84 E-03$ & $5.70 E-01$ & $3.93 \mathrm{E}-02$ & $3.49 E-01$ \\
\hline Sr90 & $6.74 E+01$ & $9.61 E+01$ & $3.67 \mathrm{E}+02$ & $1.51 \mathrm{E}-02$ & & $2.35 \mathrm{E}-02$ & & $5.50 \mathrm{E}+02$ & $3.59 E+02$ & $6.38 E+00$ & $2.23 E+03$ & $2.87 E+02$ \\
\hline Y90 & $6.74 \mathrm{E}+01$ & $9.61 E+01$ & $3.67 E+02$ & $1.51 E-02$ & & $2.35 \mathrm{E}-02$ & & $5.50 \mathrm{E}+02$ & $3.59 E+02$ & $6.38 E+\infty$ & $2.23 E+03$ & $2.87 \mathrm{E}+02$ \\
\hline Tc9s & & $5.78 E+\infty 0$ & $1.46 \mathrm{E}+01$ & $2.28 E-03$ & & $4.20 E-03$ & & $2.88 E+01$ & $6.16 \mathrm{E}-03$ & $1.79 E+\infty$ & $9.96 \mathrm{E}-02$ & $7.55 E+00$ \\
\hline Cs137 & & $1.45 E+04$ & $3.64 E+04$ & $5.96 E+00$ & & $1.12 \mathrm{E}+01$ & & $2.05 E+04$ & $1.61 \mathrm{E}+01$ & $5.01 E+03$ & $2.60 E+02$ & $5.45 \mathrm{E}+03$ \\
\hline Ba137 & & $1.38 \mathrm{E}+04$ & $3.46 \mathrm{E}+04$ & $5.66 \mathrm{E}+00$ & & $1.06 \mathrm{E}+01$ & & $1.95 \mathrm{E}+04$ & $1.53 E+01$ & $4.76 E+03$ & $2.47 E+02$ & $5.18 E+03$ \\
\hline Np237 & & 9.05E-03 & $1.95 \mathrm{E}-02$ & & & & & 1.97E-02 & & $1.34 E-03$ & & $5.28 E-03$ \\
\hline Pu239 & 1.21E-01 & $2.52 E-02$ & & & & & & & 1.98E-01 & & $3.49 E+00$ & $3.82 E-02$ \\
\hline Pu240 & $1.63 \mathrm{E}-02$ & $6.38 \mathrm{E}-03$ & & & & & & & $6.12 \mathrm{E}-02$ & & $7.42 \mathrm{E}-01$ & $8.14 E-03$ \\
\hline Pu241 & $9.78 \mathrm{E}-02$ & $1.08 \mathrm{E}-01$ & & & & $2.82 \mathrm{E}-03$ & & & $1.65 \mathrm{E}+00$ & $2.84 E-03$ & $1.08 \mathrm{E}+04$ & 1.01E-01 \\
\hline Am 241 & 1.69E-01 & 5.47E-01 & 3.44E-01 & & & $1.11 \mathrm{E}-02$ & & $9.75 E+00$ & $4.97 \mathrm{E}-01$ & $1.71 \mathrm{E}-02$ & $5.73 E+\infty 0$ & $2.82 E+00$ \\
\hline $\mathrm{Cm} 244$ & & & & & & & & $1.98 E-03$ & & & & \\
\hline Total & $1.35 \mathrm{E}+02$ & $2.85 E+04$ & $7.18 \mathrm{E}+04$ & 1.17E+01 & & $2.19 \mathrm{E}+01$ & & $4.12 \mathrm{E}+04$ & $7.52 \mathrm{E}+02$ & $9.79 E+03$ & $4.98 \mathrm{E}+0.3$ & $1.12 \mathrm{E}+04$ \\
\hline & & & & & & & & & & & & \\
\hline & & & & & & & & & & & & \\
\hline & & & & & & & & & & & & \\
\hline $\begin{array}{c}\text { Insoluble } \\
\text { (Ci) }\end{array}$ & TY-106 & U-101 & $\mathrm{U}-102$ & U-103 & U. 104 & U-105 & U-106 & U. 107 & $U-108$ & U-109 & $U-110$ & U-111 \\
\hline C14 & & 1.90E-02 & $4.71 \mathrm{E}-02$ & & & & & $1.41 \mathrm{E}-02$ & & $5.76 \mathrm{E}-03$ & & $3.53 \mathrm{E}-03$ \\
\hline Sr9o & $6.68 \mathrm{E}+03$ & $9.52 \mathrm{E}+03$ & $3.64 \mathrm{E}+04$ & $1.50 E+\infty 0$ & & $2.32 \mathrm{E}+00$ & 1.49E-03 & $5.44 E+04$ & $3.56 \mathrm{E}+04$ & $6.32 E+02$ & $2.21 E+05$ & $2.84 E+04$ \\
\hline Y90 & $6.68 \mathrm{E}+03$ & $9.52 \mathrm{E}+03$ & $3.64 E+04$ & $1.50 \mathrm{E}+00$ & & $2.32 E+00$ & $1.49 E-03$ & $5.44 E+04$ & $3.56 \mathrm{E}+04$ & $6.32 \mathrm{E}+02$ & $2.21 E+05$ & $2.84 E+04$ \\
\hline Tc99 & & $5.78 \mathrm{E}+\infty 0$ & $1.46 \mathrm{E}+01$ & $2.28 \mathrm{E}-03$ & & $4.20 \mathrm{E}-03$ & & $2.88 \mathrm{E}+01$ & $6.16 \mathrm{E}-03$ & $1.79 E+\infty$ & $9.96 \mathrm{E}-02$ & $7.55 \mathrm{E}+00$ \\
\hline$C \leq 137$ & & $463 E+03$ & $1.165+04$ & $1.905+00$ & & $3.585 \div+\infty$ & & $5.565 \div 03$ & $5.165 \div 00$ & $1.605 \div \infty$ & $8.30 E \div 01$ & $1.745 \div 03$ \\
\hline Ba137 & & $4.40 \mathrm{E}+03$ & $1.10 \mathrm{E}+04$ & $1.81 E+\infty$ & & $3.40 \mathrm{E}+00$ & & $6.23 \mathrm{E}+03$ & $4.90 \mathrm{E}+00$ & $1.52 E+03$ & $7.88 E+01$ & $1.65 E+03$ \\
\hline Np237 & & $8.15 \mathrm{E}-02$ & 1.75E-01 & & & & & $1.78 E-01$ & & $1.20 \mathrm{E}-02$ & $2.04 E-03$ & $4.75 E-02$ \\
\hline Pu239 & $5.92 \mathrm{E}+\infty 0$ & $1.24 E+00$ & & & & $3.59 E-02$ & & & $9.68 \mathrm{E}+00$ & 4.67E-02 & $1.71 E+02$ & $1.87 E+00$ \\
\hline Pu240 & $7.43 E-01$ & 2.91E-01 & & & & $8.91 \mathrm{E}-03$ & & & $2.80 \mathrm{E}+00$ & $9.68 \mathrm{E}-03$ & $3.39 \mathrm{E}+01$ & $3.72 \mathrm{E}-01$ \\
\hline Pu241 & $1.43 E+\infty$ & $1.58 E+\infty$ & & & & $4.14 E-02$ & & & $2.42 E+01$ & $4.17 \mathrm{E}-02$ & $1.59 \mathrm{E}+02$ & $1.48 \mathrm{E}+00$ \\
\hline Am241 & $1.55 \mathrm{E}+\infty$ & $5.02 E+\infty$ & $3.16 \mathrm{E}+00$ & & & $1.02 \mathrm{E}-01$ & & $8.94 E+01$ & $4.56 \mathrm{E}+00$ & 1.57E-01 & $5.25 \mathrm{E}+01$ & $2.58 E+01$ \\
\hline $\mathrm{Cm} 244$ & & $5.40 \mathrm{E}-03$ & $1.45 \mathrm{E}-02$ & & & & & $1.96 \mathrm{E}-01$ & & 1.47E-03 & & $4.99 \mathrm{E}-02$ \\
\hline Total & $1.34 \mathrm{E}+04$ & $2.81 \mathrm{E}+04$ & $9.54 \mathrm{E}+04$ & $6.73 \mathrm{E}+00$ & & $1.18 \mathrm{E}+01$ & $3.07 \mathrm{E}-03$ & $1.22 \mathrm{E}+05$ & $7.12 \mathrm{E}+04$ & $4.39 E+03$ & $4.42 E+05$ & $6.03 \mathrm{E}+04$ \\
\hline
\end{tabular}


Table A-2. Radionuclide Inventory Estimates for Single-Shell Tanks

\begin{tabular}{|c|c|c|c|c|c|c|}
\hline $\begin{array}{l}\text { Soluble } \\
\text { (Ci) }\end{array}$ & U.112 & U-201 & $\mathrm{U}-2 \mathrm{O} 2$ & U-203 & $U-204$ & Total \\
\hline C14 & $3.02 E+00$ & 4.89E-01 & $5.06 \mathrm{E}-01$ & $4.45 E-01$ & 1.17E-01 & $2.98 E+03$ \\
\hline Sr9o & $1.97 \mathrm{E}+01$ & $1.25 \mathrm{E}-04$ & $1.15 \mathrm{E}-01$ & $1.06 \mathrm{E}-01$ & $3.31 \mathrm{E}-02$ & $4.12 E+05$ \\
\hline Y90 & $1.97 E+01$ & $1.25 E-01$ & $1.15 E-01$ & $1.06 \mathrm{E}-01$ & $3.31 \mathrm{E}-02$ & $4.12 E+05$ \\
\hline Tc99 & $9.85 E+\infty 0$ & $1.54 \mathrm{E}+\infty 0$ & $1.61 E+\infty$ & $1.42 \mathrm{E}+\infty 0$ & $3.74 E-01$ & $8.89 E+03$ \\
\hline Cs 137 & 2.37E+04 & $3.73 E+03$ & $3.92 E+03$ & $3.46 \mathrm{E}+03$ & $9.16 E+02$ & $7.18 E+06$ \\
\hline $\mathrm{Ba} 137$ & $2.25 E+04$ & $3.55 E+03$ & $3.72 E+03$ & $3.28 E+03$ & $8.70 E+02$ & $6.82 E+06$ \\
\hline Np237 & $1.44 E-02$ & 2.27E-03 & 2.39E-03 & 2.09E-03 & & $6.95 E+00$ \\
\hline Pu239 & 4.24E-03 & & & & & $3.44 E+02$ \\
\hline Pu240 & & & & & & $9.15 E+01$ \\
\hline Pu241 & $1.12 \mathrm{E}-02$ & & & & & $2.29 \mathrm{E}+03$ \\
\hline Am241 & $2.92 \mathrm{E}-02$ & & & & & $2.65 E+03$ \\
\hline $\mathrm{Cm} 244$ & & & & & & $1.20 E+00$ \\
\hline Total & $4.62 E+04$ & $7.28 E+03$ & $7.65 E+03$ & $6.74 E+03$ & $1.79 E+03$ & $1.48 E+07$ \\
\hline & & & & & & \\
\hline & & & & & & \\
\hline & & & & & & \\
\hline $\begin{array}{l}\text { Insoluble } \\
\text { (Ci) }\end{array}$ & $U-112$ & U-201 & $U-202$ & U-203 & U-204 & Total \\
\hline C14 & $3.05 E-02$ & 4.94E-03 & 5.11E-03 & $4.50 \mathrm{E}-03$ & $1.18 \mathrm{E}-03$ & $3.01 \mathrm{E}+01$ \\
\hline Sr9o & $1.95 E+03$ & $1.24 E+01$ & $1.14 E+01$ & $1.05 \mathrm{E}+01$ & $3.28 E+00$ & $4.08 E+07$ \\
\hline Y90 & $1.95 E+03$ & $1.24 E+01$ & $1.14 \mathrm{E}+01$ & $1.05 \mathrm{E}+01$ & $3.28 \mathrm{E}+00$ & $4.08 E+07$ \\
\hline Tc99 & $9.85 E+00$ & $1.54 E+00$ & $1.61 \mathrm{E}+00$ & $1.42 E+00$ & $3.74 \mathrm{E}-01$ & $8.89 E+03$ \\
\hline$C_{5137}$ & $7.565 \div 03$ & $1.195 \div 03$ & $1.25 E \div 03$ & $1.405 \div 03$ & $2.035 \div 02$ & $2.205 \div 06$ \\
\hline Ba137 & $7.18 \mathrm{E}+03$ & $1.13 E+03$ & $1.19 \mathrm{E}+03$ & $1.05 E+03$ & $2.78 E+02$ & $2.18 E+06$ \\
\hline Np237 & $1.30 \mathrm{E}-01$ & 2.04E-02 & $2.15 E-02$ & $1.88 \mathrm{E}-02$ & $5.00 E-03$ & $6.25 \mathrm{E}+01$ \\
\hline Pu239 & $2.08 E-01$ & & & & & $1.69 \mathrm{E}+04$ \\
\hline Pu240 & $4.13 E-02$ & & & & & $4.18 E+03$ \\
\hline Pu241 & $1.64 \mathrm{E}-01$ & & & & & $3.36 E+04$ \\
\hline Am241 & $2.68 \mathrm{E}-01$ & & & & & $2.43 E+04$ \\
\hline $\mathrm{Cm} 244$ & 2.57E-03 & & & & & $1.19 \mathrm{E}+02$ \\
\hline Total & $1.87 E+04$ & $2.35 E+03$ & $2.47 E+03$ & $2.18 \mathrm{E}+03$ & $5.78 E+02$ & $8.61 E+07$ \\
\hline
\end{tabular}


WHC-SD-WM-TI-784

Revision 0

Table A-3. Chemical Inventory Estimates for Double-Shell Tanks

\begin{tabular}{|c|c|c|c|c|c|c|c|c|}
\hline $\begin{array}{c}\text { Soluble } \\
\text { (kg) }\end{array}$ & AN-101 & AN-102 & AN-103 & AN-104 & AN-105 & AN-106 & AN-107 & AP- 101 \\
\hline $\mathrm{Ag}+$ & $4.57 E+00$ & & $5.75 E+01$ & & & $2.89 \mathrm{E}-01$ & & \\
\hline Al(OH)4- & $1.97 E+04$ & $1.94 E+05$ & $7.29 \mathrm{E}+0.5$ & $5.45 E+05$ & $7.09 \mathrm{E}+05$ & $2.60 E+03$ & $7.33 E+04$ & $9.42 E+00$ \\
\hline \multicolumn{9}{|l|}{$\mathrm{As}+5$} \\
\hline \multicolumn{9}{|l|}{$B+3$} \\
\hline $\mathrm{Ba}+2$ & $1.32 \mathrm{E}+00$ & $5.88 \mathrm{E}+02$ & $5.75 E+01$ & & & 8.82E-01 & $2.09 E+01$ & $9.07 E-01$ \\
\hline \multicolumn{9}{|l|}{$\mathrm{Be}+2$} \\
\hline$B i+3$ & $1.93 \mathrm{E}-02$ & & $1.72 E+03$ & & & $1.15 \mathrm{E}+01$ & & \\
\hline $\mathrm{Ca}+2$ & $1.61 E+01$ & $2.08 \mathrm{E}+03$ & $3.10 E+02$ & & & $7.17 E+\infty$ & $2.06 E+03$ & $8.06 \mathrm{E}+01$ \\
\hline$C d+2$ & $4.24 E+00$ & & $5.75 E+01$ & & & $3.94 E+00$ & & $5.74 \mathrm{E}-01$ \\
\hline \multicolumn{9}{|l|}{$\mathrm{C}_{\theta+3}$} \\
\hline $\mathrm{Cl}-$ & $1.57 E+03$ & $2.55 E+04$ & $3.45 \mathrm{E}+04$ & $3.10 \mathrm{E}+04$ & $3.65 E+04$ & $1.96 \mathrm{E}+02$ & $1.28 \mathrm{E}+04$ & $8.83 E+01$ \\
\hline $\mathrm{Co}+3$ & & $1.77 \mathrm{E}-05$ & & & & & & \\
\hline $\cos -2$ & $1.40 E+04$ & $1.90 E+05$ & $3.22 \mathrm{E}+04$ & $1.17 \mathrm{E}+05$ & $9.33 E+04$ & $1.56 \mathrm{E}+03$ & $2.08 E+05$ & $3.81 E+03$ \\
\hline $\mathrm{Cr}(\mathrm{OH}) 4$ & & $4.94 E+03$ & $8.39 E+03$ & $7.69 E+03$ & $7.98 E+03$ & $1.23 E+0.2$ & $3.14 E+03$ & 4.35E-01 \\
\hline Cst & $1.22 \mathrm{E}+01$ & $6.89 \mathrm{E}+01$ & $1.44 E+02$ & $1.63 E+02$ & $1.16 \mathrm{E}+02$ & $7.92 \mathrm{E}-01$ & $5.22 E+01$ & $7.01 \mathrm{E}-03$ \\
\hline \multicolumn{9}{|l|}{$\mathrm{Cu}+2$} \\
\hline $\mathrm{F}-$ & $3.78 \mathrm{E}+03$ & $1.54 E+04$ & $2.64 E+03$ & & & $4.89 E+00$ & $2.72 \mathrm{E}+03$ & $2.75 \mathrm{E}+02$ \\
\hline $\mathrm{Fe}+3$ & $4.00 \mathrm{E}+01$ & $6.94 \varepsilon+02$ & $2.53 E+02$ & $4.61 E+01$ & $4.79 E+01$ & 5.50E-01 & $1.99 \mathrm{E}+\mathrm{O} 3$ & $1.13 E+00$ \\
\hline $\mathrm{H} 2 \mathrm{O}$ & $5.24 E+05$ & $6.25 E+06$ & $9.49 E+06$ & $8.60 E+06$ & $8.81 E+06$ & $5.54 E+04$ & $5.99 E+06$ & $1.53 E+05$ \\
\hline $\mathrm{Hg}+2$ & $1.34 \mathrm{E}-02$ & & $5.75 E+01$ & & & 3.97E-03 & & 2.01E-02 \\
\hline $\mathrm{K}+$ & $1.50 E+03$ & $7.17 E+03$ & $5.46 \mathrm{E}+04$ & $2.95 E+04$ & $2.63 E+04$ & $8.58 E+01$ & $7.97 E+03$ & \\
\hline \multicolumn{9}{|l|}{$\mathrm{La}+3$} \\
\hline \multicolumn{9}{|l|}{$\mathrm{Mg}+2$} \\
\hline $\mathrm{Mn}+4$ & $2.52 \mathrm{E}+00$ & $3.78 E+03$ & $1.03 E+02$ & & & $4.41 \mathrm{E}+00$ & $1.44 \mathrm{E}+03$ & $6.02 \mathrm{E}-02$ \\
\hline \multicolumn{9}{|l|}{$\mathrm{Mo}+6$} \\
\hline $\mathrm{Na}+$ & $6.72 E+04$ & $8.42 E+05$ & $1.21 E+06$ & $1.14 E+06$ & $1.18 \mathrm{E}+06$ & $7.18 \mathrm{E}+03$ & $8.12 \mathrm{E}+05$ & $1.79 \mathrm{E}+04$ \\
\hline $\mathrm{Ni}+3$ & $1.10 \mathrm{E}+01$ & $1.44 E+03$ & $8.62 E+01$ & & & & $1.60 E+03$ & \\
\hline NO2- & $2.13 E+04$ & $2.09 E+05$ & $4.96 \mathrm{E}+05$ & $3.65 E+05$ & $5.14 E+05$ & $2.35 \varepsilon+03$ & $1.52 E+05$ & $4.45 E+03$ \\
\hline NO3- & $5.29 \mathrm{E}+04$ & $7.74 \mathrm{E}+05$ & $5.75 \mathrm{E}+05$ & $7.93 \mathrm{E}+05$ & $8.29 E+05$ & $5.37 E+03$ & $8.60 E+05$ & $6.34 \mathrm{E}+03$ \\
\hline $\mathrm{OH}-$ & $1.28 \mathrm{E}+04$ & $4.13 E+05$ & $4.52 E+05$ & $6.96 \mathrm{E}+0.5$ & $5.97 \mathrm{E}+05$ & $1.20 \mathrm{E}+03$ & $4.70 \mathrm{E}+05$ & $7.61 \mathrm{E}+03$ \\
\hline $\mathrm{Pb}+4$ & $5.36 \mathrm{E}+00$ & & $2.59 \mathrm{E}+02$ & & & $3.66 \mathrm{E}+01$ & & $1.79 \mathrm{E}+00$ \\
\hline PO4-3 & $5.26 \mathrm{E}+03$ & $3.09 \mathrm{E}+04$ & $3.33 E+03$ & $1.15 E+04$ & $8.14 E+03$ & $1.46 \mathrm{E}+03$ & $1.64 \mathrm{E}+03$ & $4.01 E+01$ \\
\hline \multicolumn{9}{|l|}{$\mathbf{R b}+$} \\
\hline \multicolumn{9}{|l|}{$\mathrm{Re}+7$} \\
\hline \multicolumn{9}{|l|}{$\mathrm{Rh}+\mathbf{3}$} \\
\hline \multicolumn{9}{|l|}{$\mathrm{Ru}+3$} \\
\hline \multicolumn{9}{|l|}{$5 \theta+6$} \\
\hline $\mathrm{Si}+4$ & $4.18 \mathrm{E}-02$ & & $2.65 E+0.3$ & & & & & \\
\hline so4-2 & $4.08 E+03$ & $3.79 E+04$ & $5.75 E+03$ & $2.70 E+04$ & $2.81 \mathrm{E}+04$ & $1.71 \mathrm{E}+\mathrm{O} 2$ & $4.32 \mathrm{E}+04$ & $3.46 \mathrm{E}+02$ \\
\hline $\mathrm{Sr}+2$ & $6.04 \mathrm{E}-03$ & $3.12 \mathrm{E}+00$ & 2.82E-01 & 2.41E-01 & $8.44 E-02$ & $9.76 \mathrm{E}-04$ & $2.48 \mathrm{E}+0 \mathrm{O}$ & $1.38 \mathrm{E}-05$ \\
\hline \multicolumn{9}{|l|}{$\mathrm{T}_{\theta}+6$} \\
\hline \multicolumn{9}{|l|}{$T h+4$} \\
\hline \multicolumn{9}{|l|}{$\mathrm{TI}+4$} \\
\hline TOC & $2.12 \mathrm{E}+03$ & $1.54 E+05$ & $2.64 E+04$ & $1.90 E+04$ & $1.67 E+04$ & 1.67E + 04 & $1.78 E+05$ & $9.51 E+01$ \\
\hline $\mathrm{UO} 2+2$ & $5.79 E+O O$ & & $5.66 \mathrm{E}+\mathrm{O} 2$ & & & $1.71 \mathrm{E}-03$ & & $5.30 E+01$ \\
\hline \multicolumn{9}{|l|}{$2 n+2$} \\
\hline $\mathrm{ZrO} 2: 2 \mathrm{H} 2 \mathrm{O}$ & $8.82 \mathrm{E}-02$ & & 2.37E + 02 & & & $6.08 \mathrm{E}+00$ & & \\
\hline Total & $7.31 \mathrm{E}+05$ & $9.15 E+06$ & $1.31 E+07$ & $1.24 E+07$ & $1.29 \mathrm{E}+07$ & $9.45 E+04$ & 8.83E + 06 & $1.95 E+05$ \\
\hline
\end{tabular}


WHC-SD-WM-TI-784

Revision 0

Table A-3. Chemical Inventory Estimates for Double-Shell Tanks

\begin{tabular}{|c|c|c|c|c|c|c|c|c|}
\hline $\begin{array}{c}\text { Soluble } \\
\text { (kg) }\end{array}$ & AP-102 & AP- 103 & AP- 104 & AP- 105 & AP- 106 & AP- 107 & AP- 108 & AW-101 \\
\hline $\mathrm{Ag}+$ & 5.22E-01 & $1.71 \mathrm{E}-01$ & $6.62 \mathrm{E}-03$ & $4.31 \mathrm{E}-01$ & $2.72 \mathrm{E}+01$ & 2.07E-01 & $7.20 E+00$ & $1.36 E+02$ \\
\hline Al(OH)4 & $1.71 E+05$ & $3.71 E+03$ & 2.33E-01 & $1.28 \mathrm{E}+05$ & 3.17E+03 & $1.57 E+01$ & $3.11 E+04$ & $4.00 E+05$ \\
\hline$A s+5$ & & & & & & & & 4.06E-02 \\
\hline \multicolumn{9}{|l|}{$B+3$} \\
\hline $\mathrm{Ba}+2$ & $1.19 E+00$ & $2.78 \mathrm{E}-01$ & $3.30 \mathrm{E}-\mathrm{O} 2$ & $1.98 \mathrm{E}+00$ & 6.87E-01 & $1.04 \mathrm{E}-01$ & $2.08 E+00$ & $3.80 E+01$ \\
\hline \multicolumn{9}{|l|}{$\mathrm{Be}+2$} \\
\hline $\mathrm{Bi}+3$ & & & $1.98 \mathrm{E}-01$ & & & & $3.04 \mathrm{E}-02$ & $4.93 E+02$ \\
\hline $\mathrm{Ca}+2$ & & & $5.95 \mathrm{E}-02$ & $2.07 \mathrm{E}+02$ & & $8,44 E+01$ & $2.53 E+01$ & $1.35 E+02$ \\
\hline $\mathrm{Cd}+2$ & $6.14 \mathrm{E}+00$ & 4.75E-01 & $1.32 \mathrm{E}-02$ & $5.49 \mathrm{E}+00$ & $2.53 \mathrm{E}+01$ & $8.40 \mathrm{E}-02$ & $6.69 E+00$ & $4.93 E+00$ \\
\hline \multicolumn{9}{|l|}{$\mathrm{Ce}+3$} \\
\hline $\mathrm{Cl}-$ & $1.21 E+04$ & $2.12 E+02$ & $2.49 E+00$ & $7.32 E+03$ & $2.40 E+02$ & $9.24 \mathrm{E}+01$ & $2.48 E+03$ & $2.11 E+04$ \\
\hline $\mathrm{Co}+3$ & & & & & & & & 5.90E-O5 \\
\hline $\cos -2$ & $1.12 E+05$ & $1.10 \mathrm{E}+04$ & & $6.98 E+04$ & $1.10 \mathrm{E}+04$ & $6.22 \mathrm{E}+03$ & $2.20 E+04$ & \\
\hline $\mathrm{Cr}(\mathrm{OH}) 4$ & $7.12 \mathrm{E}+03$ & $5.18 \mathrm{E}+01$ & 3.1OE-OI & $1.59 \mathrm{E}+03$ & $5.57 \mathrm{E}+01$ & $3.57 \mathrm{E}-01$ & $1.15 \mathrm{E}+03$ & $1.80 E+03$ \\
\hline Cst & $5.15 E-01$ & $1.51 \mathrm{E}+00$ & & $3.81 E+01$ & $1.05 \mathrm{E}+00$ & $2.79 \mathrm{E}-05$ & $1.72 E+01$ & $1.01 E+02$ \\
\hline $\mathrm{Cu}+2$ & & & & & & & & $9.86 \mathrm{E}+01$ \\
\hline F. & $7.01 E+02$ & $5.52 \mathrm{E}+02$ & $1.13 E+\infty$ & $4.72 E+03$ & $7.39 \mathrm{E}+\mathrm{O} 2$ & $5.97 \mathrm{E}+02$ & $5.95 E+03$ & $3.11 \mathrm{E}+02$ \\
\hline $\mathrm{Fe}+3$ & $1.59 \mathrm{E}+01$ & $5.27 E+00$ & $1.52 \mathrm{E}-01$ & $2.05 \mathrm{E}+01$ & $2.94 \mathrm{E}+01$ & $2.31 \mathrm{E}-01$ & $6.30 \mathrm{E}+01$ & $1.79 E+02$ \\
\hline $\mathrm{H} 2 \mathrm{O}$ & $3.27 E+06$ & $1.71 E+05$ & $6.37 E+03$ & $3.95 E+06$ & $1.80 E+05$ & $7.75 \mathrm{E}+04$ & $8.28 \mathrm{E}+05$ & $7.49 E+06$ \\
\hline $\mathrm{Hg}+2$ & $2.09 \mathrm{E}-02$ & $2.14 E-02$ & & $7.76 \mathrm{E}-02$ & $1.07 \mathrm{E}-02$ & $1.26 \mathrm{E}-02$ & $2.11 \mathrm{E}-02$ & $6.40 \mathrm{E}-01$ \\
\hline $\mathbf{K}+$ & $5.39 E+03$ & $6.51 \mathrm{E}+03$ & $6.62 \mathrm{E}-01$ & $9.62 E+04$ & $3.49 \mathrm{E}+03$ & & $2.36 \mathrm{E}+03$ & $1.70 E+05$ \\
\hline \multicolumn{9}{|l|}{$\mathrm{La}+3$} \\
\hline \multicolumn{9}{|l|}{$\mathrm{Mg}+2$} \\
\hline $\mathrm{Mn}+4$ & & $1.85 \mathrm{E}-01$ & 7.97E-03 & & & $3.25 \mathrm{E}-01$ & $3.96 \mathrm{E}+00$ & $1.06 \mathrm{E}+02$ \\
\hline \multicolumn{9}{|l|}{$\mathrm{Mo}+6$} \\
\hline $\mathrm{Ne}+$ & $4.26 E+05$ & $2.27 E+04$ & $7.70 \mathrm{E}+02$ & $5.18 \mathrm{E}+05$ & $2.36 \mathrm{EE}+04$ & $9.58 \mathrm{E}+03$ & $1.06 \mathrm{E}+05$ & $9.90 E+05$ \\
\hline $\mathrm{Ni}+3$ & $1.11 E+02$ & & $1.32 \mathrm{E}-02$ & $3.38 E+01$ & $1.74 E+00$ & & $1.73 E+01$ & \\
\hline NO2- & $1.59 E+05$ & $5.57 E+03$ & & $1.50 E+05$ & $4.95 \mathrm{E}+03$ & $4.20 E+03$ & $3.35 \mathrm{E}+04$ & $4.15 E+05$ \\
\hline NO3- & $3.26 E+05$ & $1.73 E+04$ & & $5.12 \mathrm{E}+05$ & $1.81 E+04$ & $4.29 E+03$ & $8.31 E+04$ & $9.21 E+05$ \\
\hline $\mathrm{OH}-$ & $6.29 E+04$ & $5.69 E+03$ & & $1.73 E+05$ & $7.57 \mathrm{E}+03$ & $4.07 E+02$ & $2.41 E+04$ & $3.24 E+05$ \\
\hline $\mathrm{Pb}+4$ & $1.37 E+01$ & $1.71 E+00$ & $2.64 \mathrm{E}-01$ & $1.67 E+01$ & $6.62 E+00$ & $1.38 E+00$ & $8.44 E+00$ & $1.23 \mathrm{E}+03$ \\
\hline PO4-3 & $4.84 \mathrm{E}+04$ & $5.95 E+02$ & $1.10 E+03$ & $1.37 E+03$ & $9.01 E+02$ & $4.20 E+01$ & $9.59 \mathrm{E}+02$ & $8.59 E+03$ \\
\hline \multicolumn{9}{|l|}{$\mathrm{Rb}+$} \\
\hline \multicolumn{9}{|l|}{$R e+7$} \\
\hline \multicolumn{9}{|l|}{$\mathrm{Rh}+3$} \\
\hline \multicolumn{9}{|l|}{$R u+3$} \\
\hline$S e+6$ & & & & & & & & 1.35E-01 \\
\hline $5 i+4$ & & & $4.30 \mathrm{E}-01$ & $1.22 E+03$ & & & $1.43 E+02$ & $1.36 E+03$ \\
\hline So4-2 & $1.88 \mathrm{E}+04$ & $1.50 \mathrm{E}+03$ & $1.08 E+02$ & $7.51 E+03$ & $5.98 E+02$ & $8.53 E+02$ & $6.42 \mathrm{E}+03$ & $4.20 \mathrm{E}+03$ \\
\hline $\mathrm{Sr}+2$ & $3.68 \mathrm{E}-02$ & $6.86 \mathrm{E}-05$ & & $3.96 \mathrm{E}-03$ & $1.80 \mathrm{E}-05$ & & $8.51 E-03$ & $2.50 \mathrm{E}-02$ \\
\hline \multicolumn{9}{|l|}{$T_{e}+6$} \\
\hline \multicolumn{9}{|l|}{$T h+4$} \\
\hline \multicolumn{9}{|l|}{$\mathrm{Tl}+4$} \\
\hline TOC & $1.37 E+04$ & $4.79 E+02$ & $1.29 E+01$ & $8.54 E+03$ & $2.07 E+03$ & $2.18 E+02$ & $3.34 E+03$ & $1.00 E+04$ \\
\hline $\mathrm{UO} 2+2$ & $1.89 E+01$ & $2.02 E+01$ & $1.92 \mathrm{E}-02$ & $1.68 \mathrm{E}+02$ & $2.03 E+01$ & $1.27 \mathrm{E}-01$ & $9.12 \mathrm{E}+00$ & $1.24 \mathrm{E}+03$ \\
\hline \multicolumn{9}{|l|}{$Z n+2$} \\
\hline $\mathrm{ZrO} 2: 2 \mathrm{H} 2 \mathrm{O}$ & & & $9.08 \mathrm{E}-01$ & & & & $1.39 \mathrm{E}-01$ & $3.64 \mathrm{E}+\mathrm{O} 2$ \\
\hline Total & $4.63 E+06$ & $2.47 E+05$ & $8.37 E+03$ & $5.63 E+06$ & $2.57 E+05$ & $1.04 E+05$ & $1.15 E+06$ & $1.08 \mathrm{E}+07$ \\
\hline
\end{tabular}


WHC-SD-WM-TI-784

Revision 0

Table A-3. Chemical Inventory Estimates for Double-Shell Tanks

\begin{tabular}{|c|c|c|c|c|c|c|c|c|}
\hline $\begin{array}{c}\text { Soluble } \\
(\mathbf{k g})\end{array}$ & AW-102 & $A W-103$ & AW-104 & $A W-105$ & $A W-10 B$ & AY-101 & $A Y-102$ & $A Z-101$ \\
\hline $\mathrm{Ag}_{\mathrm{g}+}$ & $3.31 \mathrm{E}+00$ & & $7.16 \mathrm{E}+00$ & & $2.02 \mathrm{E}+01$ & & $2.82 E+01$ & $8.00 E+00$ \\
\hline Al $(\mathrm{OH}) 4$ & $6.38 E+03$ & $3.47 E+03$ & $3.08 E+04$ & $9.51 E+02$ & $2.48 \mathrm{E}+04$ & $1.91 E+04$ & $2.32 \mathrm{E}+01$ & $7.17 E+04$ \\
\hline \multicolumn{9}{|l|}{ As +5} \\
\hline \multicolumn{9}{|l|}{$B+3$} \\
\hline $\mathrm{Ba}+2$ & $4.82 E+00$ & & $2.70 \mathrm{E}+00$ & & $7.26 E+00$ & $1.83 E+01$ & $2.06 \mathrm{E}+00$ & $6.74 E+00$ \\
\hline \multicolumn{9}{|l|}{$\mathrm{Be}+2$} \\
\hline $\mathrm{Bi}+3$ & & & $3.02 \mathrm{E}-02$ & & & & & \\
\hline $\mathrm{Ca}+2$ & & $3.00 E+01$ & $2.51 \mathrm{E}+01$ & $6.60 E+00$ & & $6.60 E+02$ & $1.25 E+01$ & $1.50 E+01$ \\
\hline$C d+2$ & $1.48 \mathrm{E}+00$ & & $6.64 \mathrm{E}+00$ & & $1.25 E+01$ & $3.07 \varepsilon+01$ & $3.89 \mathrm{E}-01$ & \\
\hline$C_{0}+3$ & & & & & & & & $1.46 \mathrm{E}+01$ \\
\hline $\mathrm{Cl}-$ & $1.72 \mathrm{E}+03$ & $5.82 E+02$ & $2.46 \mathrm{E}+03$ & $5.82 \mathrm{E}+02$ & $2.24 E+03$ & $1.41 E+03$ & $2.74 E+03$ & $5.09 E+02$ \\
\hline $\mathrm{Co}+3$ & & & & & & & & $9.49 \mathrm{E}-05$ \\
\hline $\mathrm{Co3}-2$ & $4.84 E+0.4$ & & $2.19 E+04$ & $1.20 E+04$ & $1.54 E+05$ & $5.17 E+04$ & & $1.07 E+05$ \\
\hline $\mathrm{Cr}(\mathrm{OH}) 4-$ & $1.09 E+02$ & $3.22 \mathrm{E}+01$ & & $1.75 E+01$ & $2.66 E+02$ & $9.77 E+01$ & $1.51 \mathrm{E}+\mathrm{O} 2$ & $5.59 E+03$ \\
\hline $\mathrm{Cs}+$ & $2.58 E+00$ & $1.88 \mathrm{E}+00$ & $2.14 E+01$ & $5.03 E+00$ & $1.15 \mathrm{E}+01$ & 8.67E + 00 & 3.36E-01 & $2.75 E+02$ \\
\hline \multicolumn{9}{|l|}{$\mathrm{Cu}+2$} \\
\hline $\mathrm{F}-$ & $1.49 E+04$ & $1.21 \mathrm{E}+05$ & $5.91 E+03$ & $1.19 \mathrm{E}+05$ & $2.74 \mathrm{E}+04$ & $1.70 \mathrm{E}+\mathrm{O} 3$ & $6.58 \mathrm{E}+02$ & $5.56 \mathrm{E}+03$ \\
\hline $\mathrm{Fe}+3$ & $1.17 E+02$ & & & & $3.14 \mathrm{E}+\mathrm{O} 2$ & $3.16 E+01$ & $1.22 E+00$ & \\
\hline $\mathrm{H} 2 \mathrm{O}$ & $5.31 \mathrm{E}+05$ & $4.22 E+06$ & $8.19 E+05$ & $2.69 \mathrm{E}+06$ & $1.42 \mathrm{E}+06$ & $8.34 E+06$ & $6.48 E+05$ & $2.87 E+06$ \\
\hline $\mathrm{Hg}+2$ & $1.85 \mathrm{E}-02$ & & & & $4.38 \mathrm{E}-02$ & & & \\
\hline$k+$ & & $2.65 E+04$ & $2.34 \mathrm{E}+03$ & $3.74 \mathrm{E}+04$ & & $1.02 \mathrm{E}+03$ & $1.81 E+02$ & $1.49 \mathrm{E}+04$ \\
\hline $\mathrm{La}+3$ & & & & & & $1.95 E+02$ & 1.73E-01 & $1.15 \mathrm{E}+01$ \\
\hline \multicolumn{9}{|l|}{$\mathrm{Mg}+2$} \\
\hline $\mathrm{Mn}+4$ & $3.60 \mathrm{E}+00$ & & $3.93 E+00$ & & $2.81 E+01$ & $7.85 E+02$ & & \\
\hline \multicolumn{9}{|l|}{$\mathrm{Mo}+6$} \\
\hline $\mathrm{Na}+$ & $6.22 \mathrm{E}+04$ & $2.02 E+05$ & $1.05 E+05$ & $1.49 E+05$ & $1.67 E+05$ & $1.73 E+05$ & $9.47 E+03$ & $3.73 E+05$ \\
\hline $\mathrm{Ni}+3$ & & & $1.72 E+01$ & & & $1.98 \mathrm{E}+02$ & $3.96 \mathrm{E}-01$ & \\
\hline NO2- & & $1.38 E+03$ & $3.32 E+04$ & $2.43 E+03$ & & $3.56 \mathrm{E}+04$ & $3.45 \mathrm{E}+03$ & $1.22 \mathrm{E}+05$ \\
\hline NO3- & & $7.68 \mathrm{E}+03$ & $8.26 \mathrm{E}+04$ & $7.04 E+03$ & & $1.55 \mathrm{E}+05$ & $7.52 E+02$ & $3.60 E+05$ \\
\hline $\mathrm{OH}-$ & & $5.13 E+04$ & $1.99 E+04$ & $1.76 \mathrm{E}+04$ & & $6.55 E+04$ & $7.48 \mathrm{E}+03$ & $5.17 E+04$ \\
\hline $\mathrm{Pb}+4$ & $2.96 \mathrm{E}+01$ & & & & & 2.3OE +02 & & $8.46 \mathrm{E}+00$ \\
\hline $\mathrm{PO} 4-3$ & $6.48 \mathrm{E}+02$ & & $8.23 E+03$ & & $3.06 E+03$ & $2.96 E+03$ & $3.85 E+02$ & $5.18 E+03$ \\
\hline \multicolumn{9}{|l|}{$\mathrm{Rb}+$} \\
\hline \multicolumn{9}{|l|}{$R \theta+7$} \\
\hline \multicolumn{9}{|l|}{$\mathrm{Rh}+3$} \\
\hline \multicolumn{9}{|l|}{$R u+3$} \\
\hline \multicolumn{9}{|l|}{ Se+6 } \\
\hline $\mathrm{Si}+4$ & & $1.63 E+03$ & $6.54 \mathrm{E}-02$ & $7.04 E+02$ & & $1.05 E+02$ & $1.85 E+03$ & $1.89 \mathrm{E}+03$ \\
\hline SO4-2 & $8.65 E+03$ & & $6.38 E+03$ & $9.86 \mathrm{E}+02$ & $1.44 E+04$ & $1.91 E+04$ & $3.87 E+02$ & $6.16 \mathrm{E}+04$ \\
\hline$S r+2$ & $8.68 \mathrm{E}-04$ & & $1.06 \mathrm{E}-02$ & & $7.57 \mathrm{E}-04$ & $6.46 \mathrm{E}+00$ & $6.12 \mathrm{E}-01$ & $1.90 \mathrm{E}+00$ \\
\hline \multicolumn{9}{|l|}{$\mathrm{Te}+6$} \\
\hline \multicolumn{9}{|l|}{$T h+4$} \\
\hline \multicolumn{9}{|l|}{$\mathrm{TI}+4$} \\
\hline TOC & $1.82 E+03$ & $8.15 E+02$ & $3.31 E+03$ & $4.60 E+03$ & $4.41 E+03$ & $1.75 E+04$ & $1.19 \mathrm{E}+03$ & $2.36 \mathrm{E}+\mathrm{O} 2$ \\
\hline $\mathrm{UO} 2+2$ & 7.31E-05 & & $9.05 E+00$ & & & $7.68 \mathrm{E}+01$ & $1.81 E+03$ & $2.12 E+O 3$ \\
\hline \multicolumn{9}{|l|}{$Z n+2$} \\
\hline $\mathrm{ZrO} 2: 2 \mathrm{H} 2 \mathrm{O}$ & & & $1.38 \mathrm{E}-01$ & & & $2.47 E+02$ & 5.20E-01 & \\
\hline Total & $6.76 E+05$ & $4.63 E+06$ & $1.14 \mathrm{E}+06$ & $3.04 E+06$ & $1.82 E+06$ & $8.89 E+06$ & $6.79 E+05$ & $4.06 \mathrm{E}+06$ \\
\hline
\end{tabular}


WHC-SD-WM-TI-784

Revision 0

Table A-3. Chemical Inventory Estimates for Double-Shell Tanks

\begin{tabular}{|c|c|c|c|c|c|c|c|}
\hline $\begin{array}{c}\text { Soluble } \\
(\mathbf{k} \theta)\end{array}$ & $A Z-102$ & SY-101 & SY-102 & SY-103 & $\begin{array}{l}\text { FACILITY } \\
\text { WASTE }\end{array}$ & $\begin{array}{c}\text { SALTWELL } \\
\text { LIO. RECV'D }\end{array}$ & TOTAL \\
\hline $\mathrm{Ag}+$ & $1.94 E+01$ & & $4.85 \mathrm{E}-\mathrm{O} 2$ & & $5.78 E-02$ & & $3.20 \mathrm{E}+\mathrm{O} 2$ \\
\hline Al(OH)4- & $4.00 E+04$ & $6.52 \mathrm{E}+05$ & 1.07E + 04 & $4.98 E+05$ & $2.66 \mathrm{E}+04$ & $5.36 \mathrm{E}+05$ & $4.93 E+06$ \\
\hline$A s+5$ & & & & & & & $4.06 \mathrm{E}-02$ \\
\hline$B+3$ & & & $3.43 E+01$ & & & & $3.43 E+01$ \\
\hline $\mathrm{Ba}+2$ & $4.83 E+00$ & & $9.50 \mathrm{E}-01$ & $2.93 E+01$ & & & $7.91 E+02$ \\
\hline $\mathrm{Be}+2$ & & & $2.43 \mathrm{E}-02$ & & & & $2.43 \mathrm{E}-02$ \\
\hline $\mathrm{Bi}+3$ & & & & & & & $2.23 \mathrm{E}+03$ \\
\hline $\mathrm{Ca}+2$ & $2.65 E+01$ & $3.68 E+03$ & 4.30E + 01 & $5.65 \mathrm{E}+02$ & & & $1.01 E+04$ \\
\hline $\mathrm{Cd}+2$ & & & $6.57 E+00$ & & $7.46 \varepsilon+03$ & & $7.63 E+03$ \\
\hline $\mathrm{Ce}+3$ & $8.00 E+00$ & & & & & & $2.26 \mathrm{E}+01$ \\
\hline Cl- & $2.52 \mathrm{E}+02$ & $4.82 \mathrm{E}+0.4$ & $1.81 \mathrm{E}+03$ & $2.28 E+04$ & $3.61 E+00$ & & $2.71 E+05$ \\
\hline Co +3 & $9.10 \mathrm{E}-05$ & & $1.46 E+00$ & & & & $1.46 E+00$ \\
\hline Co3-2 & $1.26 \mathrm{E}+05$ & & & $6.97 E+04$ & & $4.70 E+04$ & $1.53 E+06$ \\
\hline $\mathrm{Cr}(\mathrm{OH}) 4$ & $9.20 E+03$ & $2.72 E+03$ & $7.80 E+03$ & $3.56 E+04$ & $2.53 E+02$ & $2.71 E+04$ & $1.33 E+05$ \\
\hline $\mathrm{Cs}+$ & $1.51 E+02$ & 8.62E + 01 & $3.54 E+O O$ & $6.73 E+01$ & & $1.85 \mathrm{E}+01$ & $1.37 E+03$ \\
\hline $\mathrm{Cu}+2$ & & & 4.39E-01 & & & & $9.90 \mathrm{E}+01$ \\
\hline F. & $3.14 E+03$ & $6.95 E+02$ & $3.42 \mathrm{E}+03$ & $4.83 E+03$ & $4.09 E+03$ & $6.38 E+03$ & $3.57 E+05$ \\
\hline $\mathrm{Fe}+3$ & & $1.43 \mathrm{E}+0.2$ & $1.19 E+\infty O$ & $4.03 E+03$ & $2.68 E+03$ & & $1.07 E+04$ \\
\hline $\mathrm{H} 2 \mathrm{O}$ & $1.53 \mathrm{E}+06$ & $9.61 \mathrm{E}+06$ & $1.40 \mathrm{E}+06$ & $6.85 \mathrm{E}+06$ & $1.46 \mathrm{E}+07$ & $1.25 E+07$ & $1.39 \mathrm{E}+08$ \\
\hline $\mathrm{Hg}+2$ & & & & & & & $5.84 \mathrm{E}+01$ \\
\hline$K+$ & $4.88 E+03$ & $1.90 \mathrm{E}+0.4$ & $8.33 E+03$ & $1.07 E+04$ & $1.17 E+05$ & & $6.54 E+05$ \\
\hline $\mathrm{La}+3$ & $1.29 \mathrm{E}+01$ & & & & & & $2.20 E+02$ \\
\hline $\mathrm{Mg}+2$ & & & $1.46 \mathrm{E}-01$ & & & & $1.46 \mathrm{E}-01$ \\
\hline $\mathrm{Mn}+4$ & & & & $1.42 E+03$ & $7.10 E+02$ & & $8.39 E+03$ \\
\hline $\mathrm{Mo}+6$ & & & $7.23 E+00$ & & & & $7.23 E+00$ \\
\hline $\mathrm{Na}+$ & $1.87 \mathrm{E}+05$ & $1.19 E+O B$ & $1.17 E+05$ & 8.7OE + 05 & $2.81 E+05$ & $3.31 \mathrm{E}+06$ & $1.46 E+07$ \\
\hline $\mathrm{Ni}+3$ & & $1.70 E+0.2$ & $2.14 \mathrm{E}+00$ & $3.84 E+02$ & $7.00 E+01$ & & $4.14 E+03$ \\
\hline NO2- & $1.47 E+04$ & $2.28 \mathrm{E}+0.5$ & $2.36 E+04$ & & $1.16 \mathrm{E}+04$ & $1.48 E+06$ & $4.49 E+06$ \\
\hline NO3- & $2.17 E+04$ & $2.65 E+05$ & $1.15 E+05$ & $6.54 \mathrm{E}+05$ & $4.12 \mathrm{E}+05$ & $2.04 E+06$ & $9.90 E+06$ \\
\hline $\mathrm{OH}-$ & $4.35 E+04$ & $7.26 \mathrm{E}+0.5$ & $3.85 E+0.4$ & $3.72 \mathrm{E}+05$ & $7.73 \mathrm{E}+0.4$ & $3.67 E+05$ & $5.08 E+06$ \\
\hline $\mathrm{Pb}+4$ & $2.33 E+01$ & & $1.02 \mathrm{E}+00$ & & $4.43 E+03$ & & $6.31 E+03$ \\
\hline PO4-3 & $5.48 \mathrm{E}+02$ & $4.07 \mathrm{E}+04$ & $1.10 E+04$ & $1.57 \mathrm{E}+04$ & 8. $60 \mathrm{E}+04$ & $9.70 E+04$ & $3.96 \mathrm{E}+05$ \\
\hline \multicolumn{8}{|l|}{$\mathrm{Rb}+$} \\
\hline $\mathrm{Re}+7$ & & & $9.73 \mathrm{E}-02$ & & & & $9.73 \mathrm{E}-\mathrm{O} 2$ \\
\hline$R h+3$ & & & 8.27E-01 & & & & 8.27E-01 \\
\hline$R u+3$ & & & $1.17 \mathrm{E}+00$ & & & & $1.17 \mathrm{E}+00$ \\
\hline $\mathrm{Se}+6$ & & & & & & & $1.35 \mathrm{E}-01$ \\
\hline $\mathrm{Si}+4$ & $3.58 \mathrm{E}+03$ & & 4.03E + 02 & & $3.19 E+00$ & & $1.55 E+04$ \\
\hline SO4-2 & $4.98 E+04$ & $2.54 \mathrm{E}+04$ & $5.01 E+03$ & $1.15 E+04$ & $1.26 \mathrm{E}+03$ & & $3.91 E+05$ \\
\hline $\mathrm{Sr}+2$ & $1.18 \mathrm{E}+00$ & 3.27E-01 & & $6.24 \mathrm{E}-01$ & & $1.20 \mathrm{E}+00$ & $1.86 E+01$ \\
\hline$T_{\theta}+6$ & & & 8.27E-01 & & & & 8.27E-01 \\
\hline \multicolumn{8}{|l|}{$\mathrm{Th}+4$} \\
\hline \multicolumn{8}{|l|}{$\mathrm{Tl}+4$} \\
\hline TOC & $2.44 E+02$ & $9.44 \mathrm{E}+04$ & $7.97 \mathrm{E}+02$ & $3.31 E+04$ & $1.81 \mathrm{E}+03$ & $2.16 E+05$ & $8.31 \varepsilon+05$ \\
\hline $\mathrm{UO} 2+2$ & $3.13 E+03$ & & & & $1.44 E+01$ & & $9.26 \varepsilon+03$ \\
\hline$Z n+2$ & & & $7.78 \mathrm{E}-01$ & & & & $7.78 \mathrm{E}-01$ \\
\hline $\mathrm{ZrO} 2: 2 \mathrm{H} 2 \mathrm{O}$ & & & & & & & $8.56 \mathrm{E}+02$ \\
\hline Total & $2.04 E+06$ & $1.29 E+07$ & $1.74 \mathrm{E}+06$ & $9.45 E+06$ & $1.56 \mathrm{E}+07$ & $2.07 E+07$ & $1.83 E+08$ \\
\hline
\end{tabular}


WHC-SD-WM-TI-784

Revision 0

Table A-3. Chemical Inventory Estimates for Double-Shell Tanks

\begin{tabular}{|c|c|c|c|c|c|c|c|c|}
\hline $\begin{array}{c}\text { Insoluble } \\
(\mathbf{k g l})\end{array}$ & AN-101 & AN-102 & AN-103 & AN-104 & AN. 105 & AN-106 & AN- 107 & AP-101 \\
\hline \multicolumn{9}{|l|}{$\mathrm{Ag}+$} \\
\hline $\mathrm{Al}+\mathbf{3}$ & & $2.90 \mathrm{E}+03$ & & & & & $1.10 \mathrm{E}+03$ & \\
\hline \multicolumn{9}{|l|}{$A s+5$} \\
\hline \multicolumn{9}{|l|}{$B+3$} \\
\hline$B a+2$ & & $6.53 \mathrm{E}+01$ & & & & & $2.32 \mathrm{E}+00$ & \\
\hline \multicolumn{9}{|l|}{$\mathrm{Bo}+2$} \\
\hline \multicolumn{9}{|l|}{$B i+3$} \\
\hline $\mathrm{Ca}+2$ & & $2.31 \mathrm{E}+\mathrm{O} 2$ & & & & & $2.29 \mathrm{E}+02$ & \\
\hline \multicolumn{9}{|l|}{ Cancrinite } \\
\hline$C d+2$ & & $2.43 E+02$ & & & & & $1.91 \mathrm{E}+01$ & \\
\hline $\mathrm{C}_{\theta}+3$ & & $1.61 E+02$ & & & & & $6.44 E+01$ & \\
\hline $\mathrm{Cl}-$ & & $2.58 \mathrm{E}+\mathrm{O} 2$ & & & & & $1.29 E+02$ & \\
\hline \multicolumn{9}{|l|}{$\mathrm{Co}+3$} \\
\hline CO3-2 & & $1.92 \mathrm{E}+03$ & & & & & $2.11 E+03$ & \\
\hline $\mathrm{Cr}+3$ & & $1.13 \mathrm{E}+02$ & & & & & $7.15 E+01$ & \\
\hline $\mathrm{Cs}+$ & & $6.94 \mathrm{E}-01$ & & & & & $5.28 \mathrm{E}-01$ & \\
\hline \multicolumn{9}{|l|}{$\mathrm{Cu}+2$} \\
\hline$F-$ & & $1.56 E+02$ & & & & & $2.75 E+01$ & \\
\hline $\mathrm{Fe}+3$ & & $3.65 E+01$ & & & & & $1.04 E+02$ & \\
\hline \multicolumn{9}{|l|}{$\mathrm{Hg}+2$} \\
\hline$K+$ & & $7.24 \mathrm{E}+01$ & & & & & $8.05 E+01$ & \\
\hline $\mathrm{La}+3$ & & $5.99 E+02$ & & & & & $1.91 E+01$ & \\
\hline \multicolumn{9}{|l|}{$\mathrm{Mg}+2$} \\
\hline$M n+4$ & & $1.99 E+02$ & & & & & $7.57 E+01$ & \\
\hline \multicolumn{9}{|l|}{ Mo+6 } \\
\hline $\mathrm{Na}+$ & & $8.51 E+03$ & & & & & $8.20 \mathrm{E}+03$ & \\
\hline $\mathrm{Ni}+3$ & & $7.58 \mathrm{E}+01$ & & & & & $8.42 \mathrm{E}+01$ & \\
\hline NO2. & & $2.11 E+03$ & & & & & $1.53 E+03$ & \\
\hline NO3- & & $7.82 E+03$ & & & & & $8.69 E+03$ & \\
\hline $\mathrm{OH}-$ & & $1.24 E+04$ & & & & & $4.43 E+03$ & \\
\hline $\mathrm{Pb}+4$ & & $8.25 \mathrm{E}+02$ & & & & & $1.95 \mathrm{E}+02$ & \\
\hline PO4-3 & & $3.12 E+02$ & & & & & $1.66 \mathrm{E}+01$ & \\
\hline \multicolumn{9}{|l|}{$\mathrm{Rb}+$} \\
\hline \multicolumn{9}{|l|}{$\mathrm{Re}+7$} \\
\hline \multicolumn{9}{|l|}{$R h+3$} \\
\hline \multicolumn{9}{|l|}{$R u+3$} \\
\hline \multicolumn{9}{|l|}{$S_{\theta}+6$} \\
\hline $\mathrm{Si}+4$ & & $1.62 \mathrm{E}+03$ & & & & & $5.17 E+00$ & \\
\hline SO4-2 & & $3.83 E+02$ & & & & & $4.36 E+02$ & \\
\hline $\mathrm{Sr}+2$ & & $1.63 \mathrm{E}-01$ & & & & & 1.30E-01 & \\
\hline \multicolumn{9}{|l|}{$T \theta+6$} \\
\hline \multicolumn{9}{|l|}{$T h+4$} \\
\hline \multicolumn{9}{|l|}{$\mathrm{TI}+4$} \\
\hline TOC & & $1.56 \mathrm{E}+0.3$ & & & & & $1.80 E+03$ & \\
\hline \multicolumn{9}{|l|}{$\mathrm{UO}_{2}+2$} \\
\hline$Z n+2$ & & & & & & & & \\
\hline $\mathrm{ZrO} 2: 2 \mathrm{H} 2 \mathrm{O}$ & & $3.84 \mathrm{E}+02$ & & & & & $1.53 E+02$ & \\
\hline Total & & $4.30 \mathrm{E}+04$ & & & & & $2.96 \mathrm{E}+04$ & \\
\hline
\end{tabular}


WHC-SD-WM-TI-784

Revision 0

Table A-3. Chemical Inventory Estimates for Double-Shell Tanks

\begin{tabular}{|c|c|c|c|c|c|c|c|c|}
\hline $\begin{array}{c}\text { Insoluble } \\
(\mathbf{k g})\end{array}$ & AP-102 & AP-103 & AP-104 & AP-105 & AP. 106 & AP-107 & AP-108 & AW-101 \\
\hline \multicolumn{9}{|l|}{$\mathrm{Ag}+$} \\
\hline$A \mathrm{I}+3$ & & & & & & & & $4.69 E+03$ \\
\hline \multicolumn{9}{|l|}{ As +5} \\
\hline \multicolumn{9}{|l|}{$B+3$} \\
\hline \multicolumn{9}{|l|}{$B a+2$} \\
\hline \multicolumn{9}{|l|}{$B e+2$} \\
\hline \multicolumn{9}{|l|}{$\mathrm{Bi}+3$} \\
\hline $\mathrm{Ca}+2$ & & & & & & & & $4.85 E+02$ \\
\hline \multicolumn{9}{|l|}{ Cancrinite } \\
\hline \multicolumn{9}{|l|}{$\mathrm{Cd}+2$} \\
\hline \multicolumn{9}{|l|}{$\mathrm{Ce}+3$} \\
\hline Cl- & & & & & & & & $3.27 E+01$ \\
\hline \multicolumn{9}{|l|}{$\mathrm{Co}+3$} \\
\hline $\mathrm{CO} 3-2$ & & & & & & & & $7.26 \mathrm{E}+02$ \\
\hline \multicolumn{9}{|l|}{$\mathrm{Cr}_{+}+3$} \\
\hline Cst & & & & & & & & $5.22 \mathrm{E}+\infty \mathrm{O}$ \\
\hline \multicolumn{9}{|l|}{$\mathrm{Cu}+2$} \\
\hline$F$ & & & & & & & & $2.32 E+04$ \\
\hline \multicolumn{9}{|l|}{$\mathrm{Fe}+3$} \\
\hline $\mathrm{Hg}+2$ & & & & & & & & $8.83 E+01$ \\
\hline$k+$ & & & & & & & & $1.73 E+03$ \\
\hline \multicolumn{9}{|l|}{$\mathrm{La}+3$} \\
\hline \multicolumn{9}{|l|}{$\mathrm{Mg}+2$} \\
\hline \multicolumn{9}{|l|}{$M n+4$} \\
\hline \multicolumn{9}{|l|}{ Mo+6 } \\
\hline \multicolumn{9}{|l|}{$\mathrm{Na}+$} \\
\hline \multicolumn{9}{|l|}{$\mathrm{Ni}+3$} \\
\hline NO2- & & & & & & & & $7.48 E+01$ \\
\hline NO3- & & & & & & & & $4.79 E+O 3$ \\
\hline $\mathrm{OH}-$ & & & & & & & & $1.91 E+04$ \\
\hline \multicolumn{9}{|l|}{$\mathrm{Pb}+4$} \\
\hline PO4-3 & & & & & & & & \\
\hline $\mathrm{Rb}+$ & & & & & & & & \\
\hline$R \theta+7$ & & & & & & & & \\
\hline$R h+3$ & & & & & & & & \\
\hline $\mathrm{Ru}+3$ & & & & & & & & \\
\hline Se+6 & & & & & & & & \\
\hline$S i+4$ & & & & & & & & \\
\hline SO4-2 & & & & & & & & \\
\hline $\mathrm{Sr}+2$ & & & & & & & & $2.48 \mathrm{E}-02$ \\
\hline$T e+6$ & & & & & & & & \\
\hline$T h+4$ & & & & & & & & \\
\hline $\mathrm{TI}+4$ & & & & & & & & \\
\hline TOC & & & & & & & & \\
\hline $\mathrm{UO} 2+2$ & & & & & & & & \\
\hline$Z n+2$ & & & & & & & & \\
\hline $\mathrm{ZrO} 2: 2 \mathrm{H} 2 \mathrm{O}$ & & & & & & & & $3.41 E+04$ \\
\hline Total & & & & & & & & $8.90 E+04$ \\
\hline
\end{tabular}


WHC-SD-WM-TI-784

Revision 0

Table A-3. Chemical Inventory Estimates for Double-Shell Tanks

\begin{tabular}{|c|c|c|c|c|c|c|c|c|}
\hline $\begin{array}{c}\text { Insoluble } \\
\text { (kg) }\end{array}$ & $A W-102$ & AW-103 & AW-104 & $A W-105$ & AW 106 & AY-101 & $A Y-102$ & $A Z-101$ \\
\hline $\mathrm{Ag}+$ & & & & & & & $1.04 E+03$ & $8.09 E+01$ \\
\hline $\mathrm{Al}+3$ & & $9.98 E+03$ & & $2.73 E+03$ & & $1.74 E+04$ & $5.49 E+03$ & $2.21 E+04$ \\
\hline \multicolumn{9}{|l|}{$A s+5$} \\
\hline \multicolumn{9}{|l|}{$B+3$} \\
\hline $\mathrm{Ba}+2$ & & $6.80 E+02$ & & $8.00 \mathrm{E}-01$ & & $1.68 E+03$ & $2.88 \mathrm{E}+\mathrm{O} 2$ & $2.18 \mathrm{E}+02$ \\
\hline \multicolumn{9}{|l|}{$\mathrm{Be}+2$} \\
\hline \multicolumn{9}{|l|}{$\mathrm{Bi}+3$} \\
\hline $\mathrm{Ca}+2$ & & $1.47 E+03$ & & $3.23 E+O 2$ & & $4.12 \mathrm{E}+03$ & $2.04 E+03$ & $4.86 E+02$ \\
\hline \multicolumn{9}{|l|}{ Cancrinite } \\
\hline $\mathrm{Cd}+2$ & & & & & & $5.03 E+02$ & $6.03 E+01$ & $1.11 \varepsilon+03$ \\
\hline $\mathrm{Ce}+3$ & & & & & & $2.20 \mathrm{E}+\mathrm{OB}$ & & $2.29 E+02$ \\
\hline $\mathrm{Cl}-$ & & $1.80 E+01$ & & $1.80 \mathrm{E}+01$ & & & $1.57 E+02$ & $2.12 \mathrm{E}+01$ \\
\hline $\mathrm{Co}+3$ & & & & & & & & $1.49 \mathrm{E}-03$ \\
\hline $\cos -2$ & & & & $3.00 E+03$ & & & & $2.19 E+04$ \\
\hline $\mathrm{Cr}+3$ & & $6.83 E+02$ & & $3.72 \mathrm{E}+02$ & & $8.70 E+03$ & 4.87E + O2 & $2.39 \mathrm{E}+02$ \\
\hline $\mathrm{Cs}+$ & & $1.88 E+00$ & & $5.03 E+00$ & & $1.53 E+00$ & $1.20 \mathrm{E}+00$ & $8.50 E+00$ \\
\hline \multicolumn{9}{|l|}{$\mathrm{Cu}+2$} \\
\hline F- & & $9.10 \varepsilon+03$ & & $8.96 \mathrm{E}+03$ & & & $1.86 \mathrm{E}+\mathrm{O} 2$ & $1.72 E+02$ \\
\hline $\mathrm{Fe}+3$ & & $1.40 E+03$ & & $5.20 \mathrm{E}+02$ & & $6.06 \mathrm{E}+04$ & $1.22 E+04$ & $2.77 E+04$ \\
\hline \multicolumn{9}{|l|}{$\mathrm{Hg}+2$} \\
\hline $\mathrm{K}+$ & & $7.48 E+03$ & & $1.06 E+04$ & & & $1.47 \mathrm{E}+02$ & $1.13 E+03$ \\
\hline $\mathrm{Le}+3$ & & $9.00 E+02$ & & $4.50 E+02$ & & $1.60 \mathrm{E}+04$ & $5.75 E+02$ & $1.13 E+03$ \\
\hline \multicolumn{9}{|l|}{$\mathrm{Mg}+2$} \\
\hline$M n+4$ & & $9.50 E+02$ & & & & $1.18 \mathrm{E}+04$ & $1.28 E+03$ & $5.96 \mathrm{E}+02$ \\
\hline \multicolumn{9}{|l|}{$\mathrm{Mo}+6$} \\
\hline $\mathrm{Na}+$ & & $7.84 E+04$ & & $5.78 \mathrm{E}+04$ & & $2.83 E+04$ & $2.79 \mathrm{E}+\mathrm{O3}$ & $1.15 E+04$ \\
\hline $\mathrm{Ni}+3$ & & $3.60 E+02$ & & $7.00 E+01$ & & $1.04 E+03$ & $4.39 \mathrm{E}+\mathrm{O} 2$ & $1.48 E+03$ \\
\hline No2- & & $3.24 E+02$ & & $5.70 E+02$ & & & $3.49 \mathrm{E}+01$ & $1.23 E+03$ \\
\hline NO3- & & $4.32 E+03$ & & $3.96 \mathrm{E}+03$ & & & $2.69 \mathrm{E}+01$ & $3.64 \mathrm{E}+03$ \\
\hline $\mathrm{OH}-$ & & $9.37 E+04$ & & $5.35 E+04$ & & $5.88 \mathrm{E}+0.5$ & 3.77E + 04 & $7.19 E+04$ \\
\hline $\mathrm{Pb}+4$ & & & & & & $1.51 E+03$ & & $1.12 \mathrm{E}+\mathrm{O} 2$ \\
\hline PO4-3 & & $5.73 E+01$ & & $4.00 E+02$ & & $1.32 \mathrm{E}+04$ & $3.89 E+00$ & $4.50 \mathrm{E}+02$ \\
\hline \multicolumn{9}{|l|}{$\mathbf{R b}+$} \\
\hline \multicolumn{9}{|l|}{$R_{\theta}+7$} \\
\hline \multicolumn{9}{|l|}{$\mathrm{Rh}+3$} \\
\hline \multicolumn{9}{|l|}{$R u+3$} \\
\hline $5 e+6$ & & & & $2.59 E+03$ & & & & $5.20 \mathrm{E}+02$ \\
\hline $\mathrm{Si}+4$ & & $6.51 E+03$ & & $2.82 E+03$ & & $1.86 \mathrm{E}+05$ & $3.95 E+03$ & $2.50 E+03$ \\
\hline $504-2$ & & & & $1.25 E+03$ & & & $2.00 E+01$ & $6.22 E+02$ \\
\hline $5 r+2$ & & 8.23E-01 & & 5.39E-01 & & $7.73 E+01$ & $1.20 \mathrm{E}+02$ & $1.87 E+02$ \\
\hline \multicolumn{9}{|l|}{ To+6 } \\
\hline \multicolumn{9}{|l|}{$\mathrm{Th}+4$} \\
\hline \multicolumn{9}{|l|}{$\mathrm{TI}+4$} \\
\hline TOC & & $8.15 E+02$ & & $4.60 E+03$ & & $4.24 E+04$ & $5.64 \mathrm{E}+03$ & $5.44 \mathrm{E}+03$ \\
\hline UO2 +2 & & $1.92 E+04$ & & $1.01 E+04$ & & & $8.89 E+02$ & $7.94 E+02$ \\
\hline \multicolumn{9}{|l|}{$\mathrm{Zn}+2$} \\
\hline $\mathrm{ZrO2}: 2 \mathrm{H} 2 \mathrm{O}$ & & $2.78 \mathrm{E}+05$ & & $1.73 E+05$ & & $4.45 \mathrm{E}+03$ & $5.26 \mathrm{E}-03$ & $2.90 \mathrm{E}+04$ \\
\hline Total & & $5.14 E+05$ & & $3.38 \mathrm{E}+05$ & & $9.88 E+05$ & $7.55 E+04$ & $2.06 \mathrm{E}+05$ \\
\hline
\end{tabular}


Revision 0

Table A-3. Chemical Inventory Estimates for Double-Shell Tanks

\begin{tabular}{|c|c|c|c|c|c|c|c|}
\hline $\begin{array}{c}\text { Insoluble } \\
(\mathbf{k g})\end{array}$ & $A Z-102$ & SY-101 & SY-102 & SY-103 & $\begin{array}{l}\text { FACILITY } \\
\text { WASTE }\end{array}$ & $\begin{array}{l}\text { SALTWELL } \\
\text { LIO. RECV'D }\end{array}$ & TOTAL \\
\hline $\mathrm{Ag}+$ & $1.96 \mathrm{E}+02$ & & $1.58 E+02$ & & & & $1.48 \mathrm{E}+03$ \\
\hline$A \mathrm{I}+3$ & $1.23 E+04$ & $5.06 E+03$ & $1.95 E+04$ & $7.45 E+03$ & & & $1.11 E+05$ \\
\hline As + 5 & & & $2.26 \mathrm{E}+00$ & & & & $2.26 E+00$ \\
\hline \multicolumn{8}{|l|}{$B+3$} \\
\hline $\mathrm{Be}+2$ & $1.56 \mathrm{E}+02$ & & $6.20 E+01$ & $3.26 \mathrm{E}+00$ & & & $3.16 E+03$ \\
\hline $\mathrm{Be}+2$ & & & $3.08 \mathrm{E}+00$ & & & & $3.08 \mathrm{E}+00$ \\
\hline \multicolumn{8}{|l|}{$\mathrm{Bi}+3$} \\
\hline $\mathrm{Ca}+2$ & $8.58 \mathrm{E}+02$ & $3.06 \mathrm{E}+02$ & $5.04 E+03$ & $6.28 \mathrm{E}+01$ & $7.17 \mathrm{E}+03$ & & $2.28 \mathrm{E}+04$ \\
\hline \multicolumn{8}{|l|}{ Cancrinite } \\
\hline $\mathrm{Cd}+2$ & $4.28 E+03$ & & $3.26 \mathrm{E}+\mathrm{O} 2$ & & & & $6.55 E+03$ \\
\hline $\mathrm{C}_{\theta}+3$ & $1.25 \mathrm{E}+\mathrm{O} 2$ & & & & & & $2.78 \mathrm{E}+03$ \\
\hline $\mathrm{Cl}-$ & $1.05 \mathrm{E}+01$ & $4.87 E+02$ & $5.76 \mathrm{E}+00$ & $2.31 E+02$ & & & $1.37 E+03$ \\
\hline $\mathrm{Co}+3$ & & & & & $4.08 E+01$ & & $4.08 E+01$ \\
\hline $\cos -2$ & $2.59 \mathrm{E}+04$ & & $1.42 E+04$ & $7.04 E+02$ & & & $7.04 E+04$ \\
\hline $\mathrm{Cr}+3$ & $3.94 \mathrm{E}+02$ & $2.12 E+04$ & $5.51 E+03$ & $8.12 \mathrm{E}+\mathrm{O} 2$ & & & $3.86 E+04$ \\
\hline $\mathrm{Cs}_{8}$ & $4.66 E+00$ & 8.72E-01 & $5.30 \mathrm{E}-02$ & $6.79 \mathrm{E}-01$ & & & $3.08 \mathrm{E}+01$ \\
\hline $\mathrm{Cu}+2$ & & & $1.88 \mathrm{E}+02$ & & & & $1.88 \mathrm{E}+02$ \\
\hline F- & $9.71 E+01$ & $7.02 \mathrm{E}+00$ & $1.40 E+01$ & $4.88 E+01$ & & & $4.20 E+04$ \\
\hline $\mathrm{Fe}+3$ & $3.11 E+04$ & $2.06 \mathrm{E}+03$ & $1.80 E+04$ & $2.12 \mathrm{E}+\mathrm{O} 2$ & $1.83 E+03$ & & $1.56 \mathrm{E}+05$ \\
\hline $\mathrm{Hg}+2$ & & & & & & & $8.83 E+01$ \\
\hline $\mathbf{K}+$ & $3.67 \mathrm{E}+02$ & $1.92 \mathrm{E}+02$ & $5.36 \mathrm{E}+03$ & $1.08 \mathrm{E}+02$ & & & $2.72 E+04$ \\
\hline $\mathrm{La}+3$ & $1.28 \mathrm{E}+03$ & & $5.32 \mathrm{E}+01$ & $9.89 E+01$ & & & $2.11 E+04$ \\
\hline $\mathrm{Mg}+2$ & & & $8.71 E+02$ & & $7.38 E+03$ & & $8.25 E+03$ \\
\hline $\mathrm{Mn}+4$ & $8.26 \mathrm{E}+02$ & & $4.79 \mathrm{E}+03$ & 7.47E + 01 & $4.09 E+04$ & & $6.15 E+04$ \\
\hline $\mathrm{Mo}+6$ & & & $3.21 E+01$ & & & & $3.21 E+01$ \\
\hline $\mathrm{Ne}+$ & $5.80 \mathrm{E}+03$ & $1.20 \mathrm{E}+04$ & $1.32 E+04$ & $8.78 E+03$ & & & $2.35 E+05$ \\
\hline $\mathrm{Ni}+3$ & $1.92 E+03$ & $9.30 \mathrm{E}+02$ & $4.53 \mathrm{E}+\mathrm{O} 2$ & $2.02 \mathrm{E}+01$ & & & $6.87 \mathrm{E}+03$ \\
\hline NO2- & $1.48 \mathrm{E}+02$ & $2.30 \mathrm{E}+03$ & $6.34 \mathrm{E}+01$ & & & & $8.40 E+03$ \\
\hline NO3- & $2.19 E+02$ & $2.68 E+03$ & $2.83 E+02$ & $6.61 \mathrm{E}+03$ & & & $4.30 E+04$ \\
\hline OH- & $5.86 E+04$ & $4.02 E+04$ & $8.19 E+04$ & $2.83 E+04$ & & & $1.09 E+06$ \\
\hline $\mathrm{Pb}+4$ & $3.09 E+02$ & & $1.36 \mathrm{E}+03$ & & & & $4.31 E+03$ \\
\hline PO4-3 & $4.77 E+01$ & $4.11 \mathrm{E}+02$ & $6.37 \mathrm{E}+03$ & $1.58 \mathrm{E}+02$ & & & $2.14 \mathrm{E}+04$ \\
\hline \multicolumn{8}{|l|}{$\mathbf{R b}+$} \\
\hline \multicolumn{8}{|l|}{$R e+7$} \\
\hline$R h+3$ & & & $1.93 \mathrm{E}+02$ & & & & $1.93 E+02$ \\
\hline \multicolumn{8}{|l|}{$\mathrm{Ru}+3$} \\
\hline $\mathrm{Se}+6$ & $2.00 \mathrm{E}+02$ & & & & & & $3.31 E+03$ \\
\hline$S i+4$ & $4.75 E+03$ & & $5.98 \mathrm{E}+03$ & $3.70 E+03$ & & & $2.18 E+05$ \\
\hline SO4-2 & $5.03 E+02$ & $2.57 E+02$ & $1.90 E+01$ & $1.16 E+02$ & $4.08 \mathrm{E}+01$ & & $3.65 E+03$ \\
\hline $\mathrm{Sr}+2$ & $1.16 \mathrm{E}+02$ & $2.48 \mathrm{E}-01$ & $9.75 E+01$ & $3.27 \mathrm{E}-03$ & & & $6.00 E+0.2$ \\
\hline \multicolumn{8}{|l|}{$\mathrm{Te}+6$} \\
\hline Th + 4 & & & $2.79 \mathrm{E}+03$ & & & & $2.79 E+03$ \\
\hline $\mathrm{TI}+4$ & & & $1.54 \mathrm{E}+02$ & & & & $1.54 \mathrm{E}+02$ \\
\hline TOC & $5.63 E+03$ & $9.54 E+02$ & $2.97 E+03$ & $3.34 \mathrm{E}+02$ & & & $7.21 E+04$ \\
\hline $\mathrm{UO} 2+2$ & $2.60 E+03$ & & & & $5.70 \mathrm{E}+03$ & & $3.93 E+04$ \\
\hline $2 n+2$ & & & $5.06 \mathrm{E}+02$ & & & & $5.06 \mathrm{E}+02$ \\
\hline $\mathrm{ZrO} 2: 2 \mathrm{H} 2 \mathrm{O}$ & $1.43 E+04$ & & $5.72 \mathrm{E}+02$ & $2.38 E+02$ & & & $5.34 E+05$ \\
\hline Total & $1.73 E+05$ & $8.90 \mathrm{E}+04$ & $1.91 E+05$ & $5.81 E+04$ & $6.31 E+04$ & & $2.86 \mathrm{E}+06$ \\
\hline
\end{tabular}


Table A-4. Radionuclide Inventory Estimates for Double-Shell Tanks

\begin{tabular}{|c|c|c|c|c|c|c|c|c|c|c|c|c|}
\hline $\begin{array}{c}\text { Soluble } \\
\text { (Ci) }\end{array}$ & AN-101 & AN-102 & AN-103 & AN-104 & AN-105 & AN-106 & AN-107 & AP-101 & AP-102 & AP-103 & AP-104 & AP-105 \\
\hline $\mathrm{C14}$ & $2.88 \mathrm{E}-01$ & & $7.09 E+00$ & & & $2.66 \mathrm{E}-02$ & & $1.87 \mathrm{E}-02$ & $1.75 E+00$ & $1.91 \mathrm{E}-02$ & & $6.75 \mathrm{E}-01$ \\
\hline Sr90 & $8.52 \mathrm{E}+\mathrm{O} 2$ & $4.38 \mathrm{E}+05$ & $3.97 E+04$ & $3.39 E+04$ & $1.19 E+04$ & $1.38 \mathrm{E}+02$ & $3.49 E+05$ & $1.95 \mathrm{E}+\infty$ & $5.19 \mathrm{E}+03$ & $9.67 E+00$ & $1.78 \mathrm{E}-03$ & $5.58 \mathrm{E}+02$ \\
\hline Y90 & $8.52 \mathrm{E}+02$ & $4.38 \mathrm{E}+05$ & $3.97 E+04$ & $3.39 E+04$ & $1.19 E+04$ & $1.38 E+02$ & $3.49 E+05$ & $1.95 E+\infty$ & $5.19 \mathrm{E}+03$ & $9.67 E+00$ & $1.78 \mathrm{E}-03$ & $5.58 E+02$ \\
\hline Tc99 & $8.77 E+01$ & $1.74 E+03$ & $6.03 E+02$ & $7.21 \mathrm{E}+02$ & $7.49 \mathrm{E}+02$ & $5.50 E+\infty$ & $1.76 E+03$ & $1.23 E-01$ & $3.58 \mathrm{E}+02$ & $4.63 \mathrm{E}+00$ & $3.46 \mathrm{E}-03$ & $2.17 E+02$ \\
\hline Cs137 & $1.97 E+05$ & $1.14 E+06$ & $2.31 E+06$ & $2.62 E+06$ & $1.87 E+06$ & $1.27 E+04$ & $8.69 E+05$ & $1.13 \mathrm{E}+02$ & $8.28 E+03$ & $2.43 E+04$ & $3.20 E-02$ & $6.12 E+05$ \\
\hline Ba137 & $1.87 \mathrm{E}+05$ & $1.08 \mathrm{E}+06$ & $2.19 E+06$ & $2.49 E+06$ & $1.77 \mathrm{E}+06$ & $1.21 E+04$ & $8.26 E+05$ & $1.07 E+02$ & $7.86 E+03$ & $2.31 E+04$ & $3.04 E-02$ & $5.81 E+05$ \\
\hline \multicolumn{13}{|l|}{ Np237 } \\
\hline Pu239 & 6.66E-01 & $7.54 E+02$ & $6.74 E+00$ & $4.16 E+01$ & $4.45 \mathrm{E}+01$ & $1.46 \mathrm{E}-03$ & $3.07 E+02$ & $8.55 E-01$ & $3.13 E-01$ & $2.99 \mathrm{E}-\mathrm{O} 2$ & & $4.91 E-01$ \\
\hline Pu240 & 1.09E-01 & $2.05 E+02$ & $1.11 E+\infty$ & $6.83 E+\infty$ & $7.30 E+\infty 0$ & & $8.35 E+01$ & $1.40 \mathrm{E}-01$ & $5.14 E-02$ & $4.90 \mathrm{E}-03$ & & $8.06 E-02$ \\
\hline Pu241 & $9.74 E-01$ & $1.15 E+03$ & $9.85 \mathrm{E}+00$ & $6.08 \mathrm{E}+01$ & $6.50 \mathrm{E}+01$ & $2.13 \mathrm{E}-03$ & $4.68 \mathrm{E}+02$ & $1.25 \mathrm{E}+00$ & $4.58 E-01$ & $4.36 \mathrm{E}-02$ & & $7.18 E-01$ \\
\hline Am241 & $7.22 E-01$ & $2.51 E+03$ & $8.08 E+00$ & $6.06 \mathrm{E}+00$ & $6.48 \mathrm{E}+00$ & $4.74 \mathrm{E}-02$ & $1.46 \mathrm{E}+03$ & $5.41 \mathrm{E}-01$ & $1.74 \mathrm{E}+00$ & $4.84 \mathrm{E}-02$ & & $1.26 E+00$ \\
\hline $\mathrm{Cm} 244$ & N/A & N/A & N/A & $\mathrm{N} / \mathrm{A}$ & $N / A$ & N/A & N/A & N/A & $N / A$ & N/A & N/A & $\mathbf{N} / \mathbf{A}$ \\
\hline Tota! & $3.86 \mathrm{E}+05$ & $3.11 \mathrm{E}+06$ & $4.58 \mathrm{E}+06$ & $5.18 \mathrm{E}+06$ & $3.67 E+06$ & $2.51 E+04$ & $2.40 E+06$ & $2.29 E+02$ & $2.69 E+04$ & 4.74E + 04 & $7.00 \mathrm{E}-02$ & $1.19 \mathrm{E}+06$ \\
\hline & & & & & & & & & & & & \\
\hline & & & & & & & & & & & & \\
\hline & & & & & & & & & & & & \\
\hline $\begin{array}{l}\text { Insoluble } \\
\text { (Ci) }\end{array}$ & AN-101 & AN-102 & AN-103 & AN-104 & AN-105 & AN-106 & AN-107 & AP-101 & AP -102 & AP- 103 & AP-104 & AP-105 \\
\hline C14 & & $8.72 \mathrm{E}+01$ & & & & & $1.88 \mathrm{E}+03$ & & & & & \\
\hline Sr90 & & $2.30 \mathrm{E}+04$ & & & & & $1.83 E+04$ & & & & & \\
\hline Y90 & & $2.30 E+04$ & & & & & $1.83 E+04$ & & & & & \\
\hline \multicolumn{13}{|l|}{ Tc99 } \\
\hline Cs 137 & & $1.15 E+04$ & & & & & $8.78 \mathrm{E}+03$ & & & & & \\
\hline Ba137 & & $1.09 \mathrm{E}+04$ & & & & & $8.34 E+03$ & & & & & \\
\hline \multicolumn{13}{|l|}{$\mathrm{Np237}$} \\
\hline Pu239 & & $3.97 \mathrm{E}+01$ & & & & & $1.62 \mathrm{E}+01$ & & & & & \\
\hline Pu 240 & & $1.01 E+01$ & & & & & $4.09 E+\infty$ & & & & & \\
\hline Pu241 & & $3.47 E+00$ & & & & & $1.42 E+\infty$ & & & & & \\
\hline Am241 & & $1.32 E+02$ & & & & & $7.68 \mathrm{E}+01$ & & & & & \\
\hline $\mathrm{Cm} 244$ & & N/A & & & & & N/A & & & & & \\
\hline Total & & $6.87 \mathrm{E}+04$ & & & & & $5.57 \mathrm{E}+04$ & & & & & \\
\hline
\end{tabular}


Table A-4. Radionuclide Inventory Estimates for Double-Shell Tanks

\begin{tabular}{|c|c|c|c|c|c|c|c|c|c|c|c|c|}
\hline $\begin{array}{l}\text { Soluble } \\
\text { (Ci) }\end{array}$ & AP-106 & AP-107 & AP-108 & AW-101 & AW- 102 & AW-103 & AW-104 & $A W-105$ & AW-106 & AY-101 & AY-102 & $A Z-101$ \\
\hline C14 & $3.31 \mathrm{E}-02$ & $1.34 \mathrm{E}-02$ & 4.06E-01 & $1.50 \mathrm{E}+00$ & & & $5.05 E-01$ & & $3.13 \mathrm{E}-01$ & & & $1.94 E+02$ \\
\hline Sr9o & $2.54 E+00$ & 4.29E-01 & $1.20 \mathrm{E}+03$ & $3.50 E+03$ & $1.22 \mathrm{E}+02$ & & $1.49 \mathrm{E}+03$ & & $1.07 E+02$ & $3.58 E+05$ & $3.70 E+03$ & $5.48 E+04$ \\
\hline$Y 90$ & $2.54 \mathrm{E}+\infty 0$ & 4.29E-01 & $1.20 \mathrm{E}+03$ & $3.50 \mathrm{E}+03$ & $1.22 \mathrm{E}+02$ & & $1.49 \mathrm{E}+03$ & & $1.07 E+02$ & $3.58 \mathrm{E}+05$ & $3.70 \mathrm{E}+03$ & $5.48 E+04$ \\
\hline To99 & $5.73 E+01$ & $8.64 E-02$ & $1.24 \mathrm{E}+02$ & $6.14 \mathrm{E}+02$ & & & $1,54 \mathrm{E}+02$ & & $7.05 E+01$ & $4.30 E+02$ & $2.47 E+01$ & $1.10 E+03$ \\
\hline Cs 137 & $1.69 E+04$ & 4.49E-01 & $2.77 E+05$ & $1.74 E+06$ & $4.15 \mathrm{E}+04$ & $4.12 \mathrm{E}+04$ & $3.45 E+05$ & $1.10 \mathrm{E}+05$ & $1.84 \mathrm{E}+05$ & $1.44 E+05$ & $5.40 \mathrm{E}+03$ & $6.14 E+06$ \\
\hline $\mathrm{Ba} 137$ & $1.61 E+04$ & 4.27E-01 & $2.63 E+05$ & $1.65 E+06$ & $3.94 E+04$ & $3.91 \mathrm{E}+04$ & $3.28 E+05$ & $1.05 \mathrm{E}+05$ & $1.75 \mathrm{E}+05$ & $1.37 \mathrm{E}+05$ & $5.13 E+03$ & $5.83 E+06$ \\
\hline \multicolumn{13}{|l|}{ Np237 } \\
\hline Pu239 & $5.81 E-01$ & $2.25 E+\infty 0$ & 9.39E-01 & $5.44 \mathrm{E}+01$ & $1.28 \mathrm{E}+00$ & & $1.17 \mathrm{E}+00$ & & $4.15 E-01$ & $2.40 E+01$ & $4.61 \mathrm{E}-01$ & $2.17 E+01$ \\
\hline Pu240 & $9.54 \mathrm{E}-02$ & 3.70E-01 & $1.54 \mathrm{E}-01$ & $8.93 E+00$ & $2.10 \mathrm{E}-01$ & & $1.92 \mathrm{E}-01$ & & $6.82 \mathrm{E}-02$ & $6.52 \mathrm{E}+00$ & $1.25 E-01$ & $6.56 E+00$ \\
\hline Pu241 & $8.49 \mathrm{E}-01$ & $3.29 \mathrm{E}+00$ & $1.37 \mathrm{E}+00$ & $7.95 \mathrm{E}+01$ & $1.87 E+00$ & & $1.71 \mathrm{E}+\infty 0$ & & $6,07 E-01$ & $3.51 E+01$ & $6.73 \mathrm{E}-01$ & $5.87 E+02$ \\
\hline Am241 & & $2.66 \mathrm{E}+00$ & $1.02 E+\infty 0$ & $1.37 E+02$ & $8.95 E-01$ & & $1.27 \mathrm{E}+\infty$ & & $8.93 E-02$ & $9.83 E+01$ & $3.60 \mathrm{E}-01$ & $5.76 E+02$ \\
\hline $\mathrm{Cm} 244$ & $\mathrm{~N} / \mathrm{A}$ & N/A & $\mathrm{N} / \mathrm{A}$ & $\mathrm{N} / \mathrm{A}$ & N/A & N/A & N/A & N/A & $\mathrm{N} / \mathrm{A}$ & N/A & N/A & N/A \\
\hline Total & $3.30 E+04$ & $8.60 E+00$ & $5.43 E+05$ & $3.40 E+06$ & $8.12 \mathrm{E}+04$ & $8.04 \mathrm{E}+04$ & $6.76 E+05$ & $2.15 E+05$ & $3.60 \mathrm{E}+05$ & $9.97 \mathrm{E}+05$ & $1.80 E+04$ & $1,21 \mathrm{E}+07$ \\
\hline & & & & & & & & & & & & \\
\hline & & & & & & & & & & & & \\
\hline & & & & & & & & & & & & \\
\hline $\begin{array}{c}\text { Insoluble } \\
\text { (Ci) }\end{array}$ & AP- 106 & AP-107 & AP- 108 & AW-101 & AW-102 & $A W-103$ & AW-104 & AW-105 & $A W-106$ & AY-101 & AY-102 & AZ-101 \\
\hline $\mathrm{C} 14$ & & & & & & & & & & & $1.81 \mathrm{E}-01$ & $1.02 E+01$ \\
\hline Sr9o & & & & & & $3.15 E+04$ & & $1.96 \mathrm{E}+04$ & & $3.96 E+05$ & $1.98 \mathrm{E}+06$ & $5.42 E+06$ \\
\hline$Y 90$ & & & & & & $3.15 E+04$ & & $1.96 \mathrm{E}+04$ & & $3.96 \mathrm{E}+05$ & $1.98 E+06$ & $5.42 \mathrm{E}+06$ \\
\hline Tc99 & & & & & & & & & & & $4.54 E+\infty$ & $2.58 E+02$ \\
\hline $\operatorname{Cs137}$ & & & & $7.21 E+04$ & & $4.12 E+04$ & & $1.10 \mathrm{E}+05$ & & $1.32 \mathrm{E}+05$ & $1.92 \mathrm{E}+04$ & $1.90 E+05$ \\
\hline Ba137 & & & & $6.85 E+04$ & & $3.91 E+04$ & & $1.05 \mathrm{E}+05$ & & $1.26 E+05$ & $1.82 E+04$ & $1.80 E+05$ \\
\hline \multicolumn{13}{|l|}{ Np237 } \\
\hline Pu239 & & & & $3.61 \mathrm{E}+01$ & & $7.97 E+02$ & & $4.19 \mathrm{E}+02$ & & $2.79 E+03$ & $6.56 E+02$ & $5.20 \mathrm{E}+02$ \\
\hline Pu240 & & & & & & $2.25 E+02$ & & $1.18 \mathrm{E}+02$ & & $6.95 \mathrm{E}+02$ & & $1.47 \mathrm{E}+02$ \\
\hline Pu241 & & & & $6.95 E+\infty$ & & $6.47 E+03$ & & $3.41 E+03$ & & $4.73 \mathrm{E}+02$ & & $4.21 E+03$ \\
\hline Am241 & & & & $9.54 \mathrm{E}+0\}$ & & $1.75 \mathrm{E}+02$ & & $1.74 \mathrm{E}+02$ & & $1.21 E+04$ & $3.25 E+03$ & $2.82 E+04$ \\
\hline $\mathrm{Cm} 244$ & & & & N/A & & $\mathrm{N} / \mathrm{A}$ & & $\mathrm{N} / \mathrm{A}$ & & N/A & N/A & N/A \\
\hline Total & & & & $1.41 \mathrm{E}+05$ & & $1.51 \mathrm{E}+05$ & & $2.58 \mathrm{E}+05$ & & $1.07 \mathrm{E}+06$ & $3.99 \mathrm{E}+06$ & $1.12 \mathrm{E}+07$ \\
\hline
\end{tabular}


Table A-4. Radionuclide Inventory Estimates for Double-Shell Tanks

\begin{tabular}{|c|c|c|c|c|c|}
\hline $\begin{array}{c}\text { Soluble } \\
\text { (Ci) }\end{array}$ & $A Z-102$ & SY-101 & SY-102 & SY-103 & Total Curies \\
\hline C14 & $1.39 \mathrm{E}+02$ & & $9.74 \mathrm{E}-01$ & & $3.46 E+02$ \\
\hline Sr9o & $2.98 E+04$ & $4.62 \mathrm{E}+04$ & $7.88 \mathrm{E}+03$ & $8.80 E+04$ & $1.47 E+06$ \\
\hline Y90 & $2.98 \mathrm{E}+04$ & $4.62 \mathrm{E}+04$ & $7.88 E+03$ & $8.80 \mathrm{E}+04$ & $1.47 \mathrm{E}+06$ \\
\hline Tc99 & $6.00 E+02$ & $2.57 \mathrm{E}+03$ & & $1.88 \mathrm{E}+03$ & $1.39 E+04$ \\
\hline Cs137 & $3.37 E+06$ & $1.43 E+06$ & $6.64 E+04$ & $1.12 E+06$ & 2.47E+07 \\
\hline Ba137 & $3.20 \mathrm{E}+06$ & $1.36 \mathrm{E}+06$ & $6.31 E+04$ & $1.06 \mathrm{E}+06$ & $2.35 E+07$ \\
\hline \multicolumn{6}{|l|}{ Np237 } \\
\hline Pu239 & $2.09 E+01$ & $8.81 E+\infty 0$ & & $1.14 E+01$ & $1.31 \mathrm{E}+03$ \\
\hline Pu240 & $6.32 \mathrm{E}+\infty$ & $2.39 E+00$ & & $3.11 E+\infty$ & $3.39 E+02$ \\
\hline Pu241 & $2.71 E+\infty$ & $1.29 \mathrm{E}+01$ & & $1.74 E+01$ & $2.50 E+03$ \\
\hline Am241 & $3.36 \mathrm{E}+02$ & $8.03 E+01$ & & $8.21 E+01$ & $5.31 \mathrm{E}+03$ \\
\hline $\mathrm{Cm} 244$ & $\bar{N} / \bar{A}$ & $\mathrm{~N} / \mathrm{A}$ & $\mathrm{N} / \mathrm{A}$ & N/A & N/A \\
\hline Total & $6.63 \mathrm{E}+06$ & $2.89 E+06$ & $1.45 \mathrm{E}+05$ & $2.36 \mathrm{E}+06$ & $5.11 E+07$ \\
\hline & & & & & \\
\hline & & & & & \\
\hline & & & & & \\
\hline $\begin{array}{l}\text { Insoluble } \\
\text { (Ci) }\end{array}$ & AZ-102 & SY-101 & SY-102 & SY-103 & Total Curies \\
\hline C.14 & $7.31 E+00$ & & $2.14 \mathrm{E}-01$ & & $1.99 E+03$ \\
\hline Sr9o & $2.95 E+06$ & $3.50 E+04$ & $4.46 \mathrm{E}+04$ & $4.61 E+02$ & $1.09 \mathrm{E}+07$ \\
\hline$Y 90$ & $2.95 \mathrm{E}+06$ & $3.50 \mathrm{E}+04$ & $4.46 \mathrm{E}+04$ & $4.61 E+02$ & $1,09 \mathrm{E}+07$ \\
\hline Te99 & $1.41 \mathrm{E}+02$ & & & & $4.04 \mathrm{E}+02$ \\
\hline Cs137 & $1.04 E+05$ & $1.45 \mathrm{E}+04$ & $9.93 \mathrm{E}+02$ & $1.13 E+04$ & $7.16 E+05$ \\
\hline Ba137 & $9.89 E+04$ & $1.37 E+04$ & $9.43 \mathrm{E}+02$ & $1.07 E+04$ & $6.80 \mathrm{E}+05$ \\
\hline \multicolumn{6}{|l|}{ Np237 } \\
\hline Pu239 & $5.01 E+02$ & $3.76 E+01$ & $2.08 E+03$ & $6.02 E-01$ & $7.89 E+03$ \\
\hline Pu240 & $1.41 E+02$ & $9.51 E+\infty$ & $7.47 \mathrm{E}+02$ & $1.52 \mathrm{E}-01$ & $2.10 \mathrm{E}+03$ \\
\hline Pu241 & $4.23 E+03$ & $1.64 E+01$ & $1.77 E+04$ & $5.27 \mathrm{E}-02$ & $3.66 \mathrm{E}+04$ \\
\hline Am241 & $1.65 \mathrm{E}+04$ & $3,42 \mathrm{E}+02$ & $1.13 \mathrm{E}+04$ & $4.32 \mathrm{E}+00$ & $7.23 \mathrm{E}+04$ \\
\hline $\mathrm{Cm} 244$ & N/A & N/A & N/A & $N / A$ & N/A \\
\hline Total & $6.13 E+06$ & $9.87 E+04$ & $1.24 E+05$ & $2.29 E+04$ & $2.34 E+07$ \\
\hline
\end{tabular}


WHC-SD-WM-TI-784

Revision 0

This page intentionally left blank. 


\begin{tabular}{|c|c|c|c|c|c|}
\hline \multirow{2}{*}{$\begin{array}{l}\text { To } \\
\text { Distribution }\end{array}$} & \multirow{2}{*}{\multicolumn{3}{|c|}{$\begin{array}{l}\text { From } \\
\text { L. W. Shelton, 376-6199 }\end{array}$}} & \multicolumn{2}{|c|}{ Page 1 of 1} \\
\hline & & & & \multicolumn{2}{|c|}{ Date $11 / 18 / 96$} \\
\hline \multicolumn{4}{|c|}{ Project Title/Work Order } & \multicolumn{2}{|c|}{ EDT No. 608755} \\
\hline \multicolumn{4}{|c|}{$\begin{array}{l}\text { Tank Waste Remediation System Baseline Tank Waste Inventory Estimates } \\
\text { for Fiscal Year 1995, WHC-SD-WM-TI-784, Rev. } 0\end{array}$} & \multicolumn{2}{|c|}{ ECN No. } \\
\hline Name & MSIN & $\begin{array}{l}\text { Text } \\
\text { With All } \\
\text { Attach. }\end{array}$ & $\begin{array}{l}\text { Text } \\
\text { Only }\end{array}$ & $\begin{array}{l}\text { Attach./ } \\
\text { Appendix } \\
\text { Only }\end{array}$ & $\begin{array}{l}\mathrm{EDT} / \mathrm{ECN} \\
\text { Only }\end{array}$ \\
\hline
\end{tabular}

Central Files

DOE Reading Room

$\begin{array}{ll}\text { A3-88 } & X \\ H 2-53 & X\end{array}$

U.S. Department of Energy-Richland Operations Office

N. R. Brown

Lockheed Martin Hanford Corp.

L. W. Shelton (6)

J. P. Sloughter

SGN Eurysis Services Corp.

P. J. Certa

Fluor Daniel Hanford Inc.

D. J. Washenfelder

Numatec Hanford Corporation

R. M. Orme

Pacific Northwest National Laboratory

L. K. Holton, Jr.
H5-27

$\mathrm{X}$

$\begin{array}{ll}\text { H5-49 } & X \\ \text { R2-54 } & X\end{array}$

H.5-61 X

S7-40 $\quad X$

K6-51 X 